\title{
Decision Phase Final Report
}

by

J. Barnes

Westinghouse Savannah River Company

Savannah River Site

Aiken, South Carolina 29808

DOE Contract No. DE-AC09-96SR18500

This paper was prepared in connection with work done under the above contract number with the U.S. Department of Energy. By acceptance of this paper, the publisher and/or recipient acknowledges the U.S. Government's right to retain a nonexclusive, royalty-free license in and to any copyright covering this paper, along with the right to reproduce and to authorize others to reproduce all or part of the copyrighted paper. 


\section{Savannah River Site}

High Level Waste Salt Disposition

Systems Engineering Team

Decision Phase Final Report (U)

WSRC-RP-99-00007

Revision: 0

November 1, 1999 
High Level Waste Salt Disposition

WSRC-RP-99-00007

Systems Engineering Team

Revision: 0

Decision Phase Final Report

Page 2 of 277

HLW Salt Disposition Systems Engineering Team

Decision Phase Final Report

Approved By:

S. F. Piccolo, HLW Salt Disposition Program Manager $\frac{11-1-99}{\text { Date }}$ 


\section{DISCLAIMER}

Portions of this document may be illegible in electronic image products. Images are produced from the best available original document. 


\section{DISCLAIMER}

This report was prepared as an account of work sponsored by an agency of the United States Government. Neither the United States Government nor any agency thereof, nor any of their employees, makes any warranty, express or implied, or assumes any legal liability or responsibility for the accuracy, completeness, or usefulness of any information, apparatus, product, or process disclosed, or represents that its use would not infringe privately owned rights. Reference herein to any specific commercial product, process, or service by trade name, trademark, manufacturer, or otherwise does not necessarily constitute or imply its endorsement, recommendation, or favoring by the United States Government or any agency thereof. The views and opinions of authors expressed herein do not necessarily state or reflect those of the United States Government or any agency thereof.

This report has been reproduced directly from the best available copy.

Available to DOE and DOE contractors from the Office of Scientific and Technical Information, P.O. Box 62, Oak Ridge, TN 37831; prices available from (615) 576-8401.

Available to the public from the National Technical Information Service, U.S. Department of Commerce, 5285 Port Royal Road, Springfield, VA 22161. 
High Level Waste Salt Disposition

Systems Engineering Team

Decision Phase Final Report
WSRC-RP-99-00007

Revision: 0

Page 4 of 277

\section{REVISION SUMMARY}

Rev. No. Rev. Date Affected Sections Description of Revision

0

$11 / 01 / 99$

N/A

Initial Issue 


\begin{abstract}
This report describes the process used and results obtained by the High Level Waste (HLW) Salt Disposition Systems Engineering Team (Team) to recommend a path forward for salt disposition at the Savannah River Site (SRS). The selection of an alternative salt disposition technology is necessary as the existing In Tank Precipitation (ITP) process cannot simultaneously meet the HLW system production and safety requirements. The SRS high level salt solution waste must be immobilized for final disposition in support of environmental protection, safety, and current and planned missions. The Team concluded that the alternative most technically suited for processing SRS high level salt solution waste within the constraints of the Federal Facilities Agreement (FFA), Site Treatment Plan (STP), SRS Tank Farm Salt/Space Management, HLW System, and DWPF interfaces is Small Tank Tetraphenylborate (TPB) Precipitation. The Team also concluded that from a DOE complex and business perspective, the Crystalline Silicotitanate (CST) Non-Elutable Ion Exchange alternative could show significant promise. With the appropriate level of research and management attention, CST Non-Elutable Ion Exchange could effectively serve the DOE complex and result in complex wide savings for technology development. CST Non-Elutable Ion Exchange can also be effectively applied to SRS waste, although with a higher project implementation risk than Small Tank TPB Precipitation.
\end{abstract}


High Level Waste Salt Disposition

Systems Engineering Team

Decision Phase Final Report
WSRC-RP-99-00007

Revision: 0

Page 6 of 277

\section{Table of Contents}

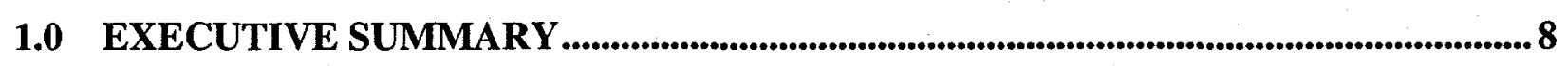

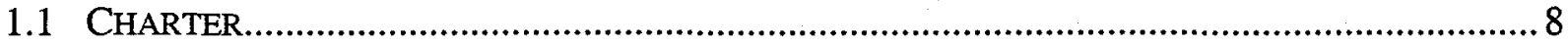

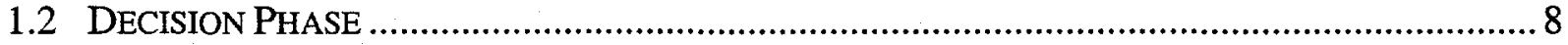

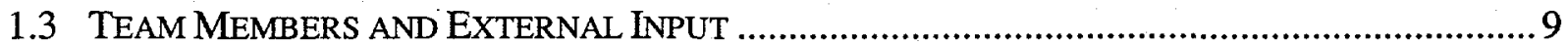

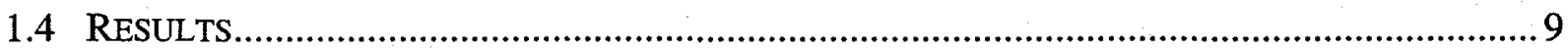

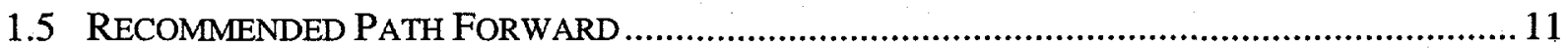

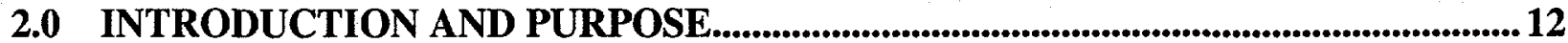

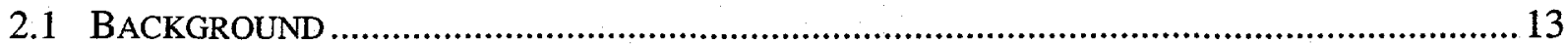

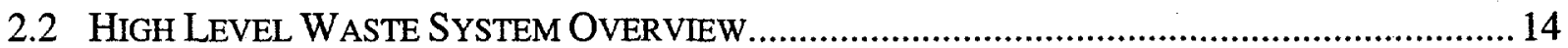

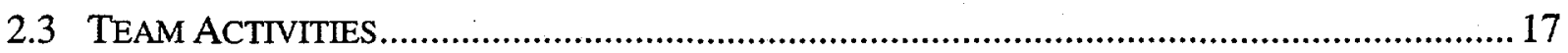

3.0 DECISION PROCESS...................................................................................................... 23

3.1 PROCESS DESCRIPTION OF THE FOUR SHORT LIST ALTERNATIVES ……............................. 23

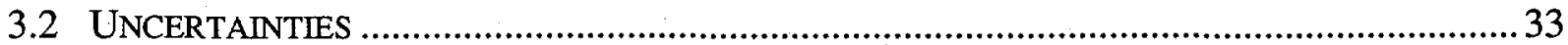

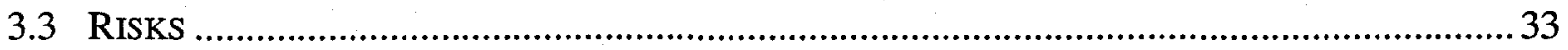

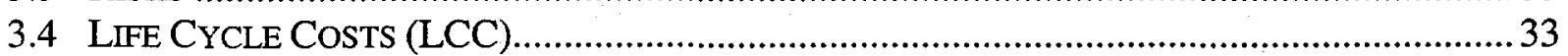

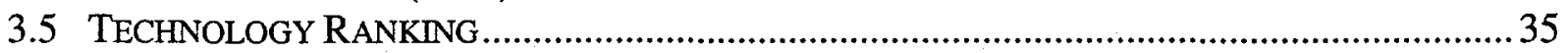

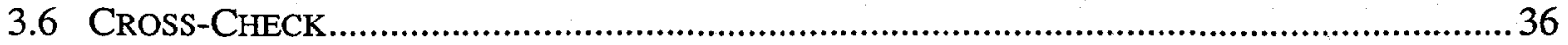

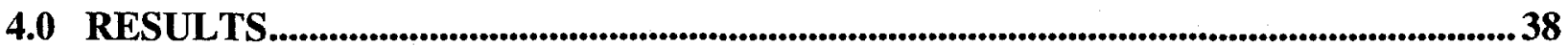

4.1 SMALl TANK TETRAPHENYLBORATE (TPB) PRECIPITATION ………................................... 38

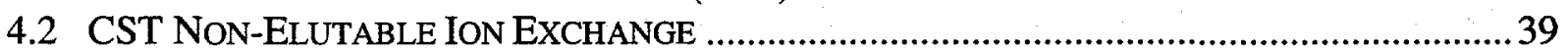

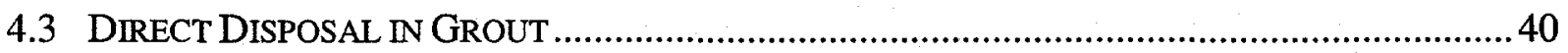

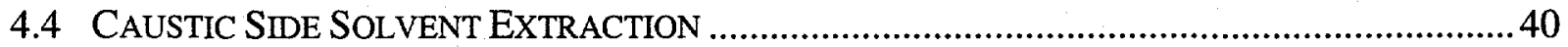

5.0 RECOMMENDED PATH FORWARD.....................................................................

6.0 ACRONYMS \& ABBREVIATIONS ........................................................................42

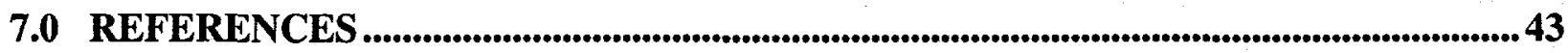

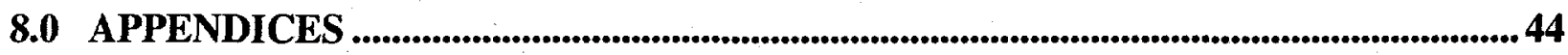

8.1 WeIGHTED EVALUATION CRITERIA AND UTILITY FunCtION VALUE FoRMS......................45

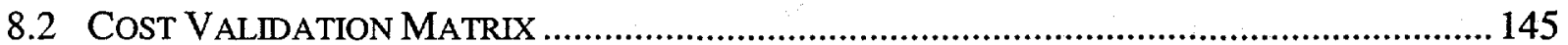

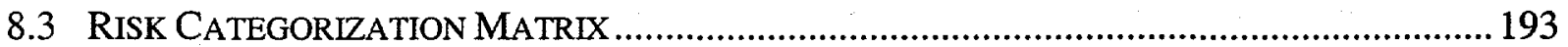

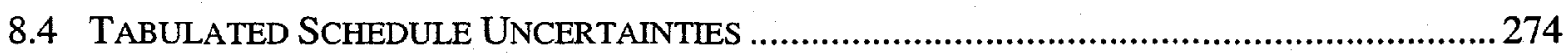




\section{Figures}

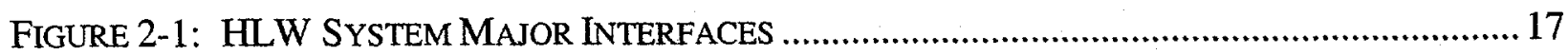

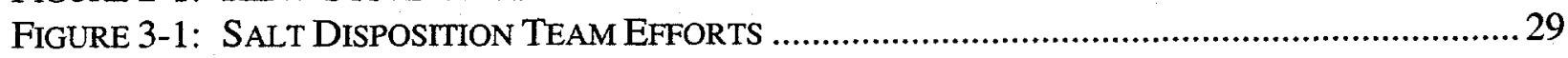

FIGURE 3-2: DECISION PHASE LOGIC DIAGRAM............................................................. 30

FIGURE 3-3: DECISION PROCESS BUSINESS MODEL …...................................................... 31

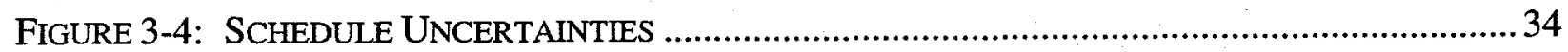

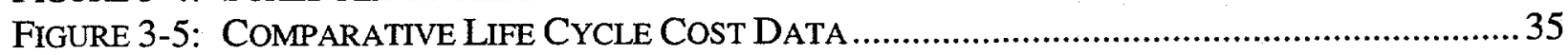

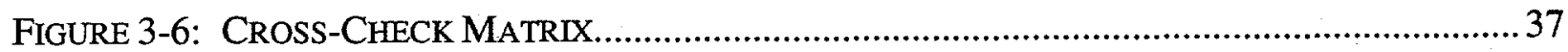

\section{Tables}

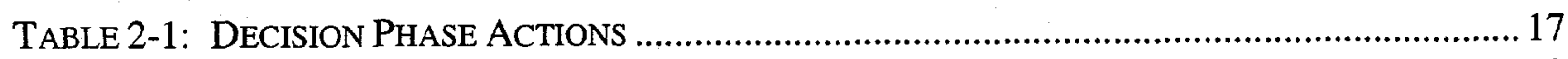

TABLE 2-2: CST ION EXCHANGE EXPERIMENT LIST .......................................................... 19

TABLE 2-3: SMALL TANK TPB PRECIPITATION EXPERIMENT LIST ..........................................20

TABLE 2-4: DIRECT DISPOSAL IN GROUT EXPERIMENT LIST...............................................2 21

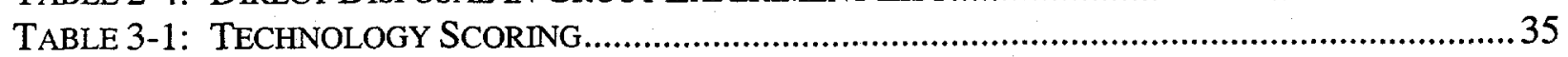




\section{$1.0 \quad$ Executive Summary}

This section provides a summary of the decision process utilized to recommend a HLW salt disposition path forward based on the HLW Salt Disposition Systems Engineering Team Decision Phase activities.

\subsection{Charter}

Westinghouse Savannah River Company (WSRC) completed a systems engineering evaluation of HLW salt disposition alternatives in October 1998 (Reference 7). The U.S. Department of Energy (DOE) - Savannah River Operations Office (SR) completed their review of the systems engineering process and recommendations on January 25, 1999 (Reference 9). The conclusions of their review and a proposed path forward were forwarded to the DOE Assistant Secretary for Environmental Management (EM-1). EM1 provided authorization to proceed with the DOE-SR proposed path forward. DOE authorized initiation of a Supplemental Environmental Impact Statement in parallel with performance of additional research on the CST Non-Elutable Ion Exchange and Small Tank TPB Precipitation technologies to address defined technical uncertainties, evaluation of tank farm salt/space management practices, selected trade studies, and additional evaluation of the regulatory/public acceptance for cesium disposal in grout.

\subsection{Decision Phase}

The Decision Phase was entered into as a continuation of the HLW Salt Disposition Systems Engineering Team efforts of 1998. The Team subjected the four short list alternatives from the 1998 Selection Phase (Caustic Side Solvent Extraction, CST NonElutable Ion Exchange, Direct Disposal in Grout, and Small Tank TPB Precipitation) to the decision process. The four alternatives were included because the Decision Phase is a continuation of the Selection Phase and each process had technical merit. Research and Development (R\&D) was conducted on CST Non-Elutable Ion Exchange and Small Tank TPB Precipitation consistent with the DOE-SR Management Plan. A more limited amount of R\&D was performed on Caustic Side Solvent Extraction and Direct Disposal in Grout. Each alternative also had some advancement in technology understanding. The decision process tools would be the same tools used in the 1998 Team activities, therefore adding results comparison validity. A decision logic was developed factoring in those attributes highlighted as issues in the WSRC Final Report, WSRC-RP-98-0170, DOE-SR Review Team Final Report, letter to James M. Owendoff dated December 16, 1998, and the Independent Project Evaluation Team Review and Assessment Report, DOE/ID-10672. Science and technology activities were performed to advance understanding for those technical uncertainties which could potentially provide technical discrimination between the alternatives. This work has been completed, and the results applied to the decision process. 
The Decision Phase selection process was baselined against the HLW Salt Disposition Systems Engineering Team's 1998 results. The decision process characterized substantive deltas in uncertainties, risks, Life Cycle Costs (LCCs), and weighted scores for the Short List alternatives. The decision process also provided a qualitative crosscheck of the decision results versus the expected conclusions from the delivered research.

\subsection{Team Members and External Input}

Decision Phase Team members were chosen to provide expertise in Program Management, Systems Engineering, Process Engineering, Operations, Research and Development, Safety Management, and Technology Integration. Members were selected to provide a strong linkage to and knowledge of the fiscal year 1998 Selection process and information. Significant WSRC engineering resources were dedicated to and managed by the Team, as was an administrative support staff. Research and Development activities were lead by the Savannah River Technology Center (SRTC), with participation from the Oak Ridge and Argonne National Laboratories, and vendor representatives.

The Team's efforts have been conducted with regular interactions with the Independent Project Evaluation (IPE) Team and the Citizen's Advisory Board (CAB) members and included a technology information exchange with Hanford. Additionally, the National Academy of Science/National Research Council held a public meeting to review the technology selection process and provided observations in a interim letter-report (reference 15). The associated input from these sources has been accommodated/addressed in this report.

\section{$1.4 \quad$ Results}

The Team concluded that the most suited technology for processing SRS high level salt solution waste within the constraints of the Federal Facilities Agreement (FFA), Site Treatment Plan (STP), SRS Tank Farm Salt/Space Management, HLW System, and DWPF interfaces is Small Tank TPB Precipitation. The research conducted has confirmed most of the targeted uncertainties in a positive nature, thus reducing the "effective" risk, and "effective" uncertainties associated with project implementation relative to its position at the end of the 1998 Selection Phase.

The safety issues raised regarding TPB decomposition in the process have been addressed in the pre-conceptual design. The process includes positive pressure nitrogen inerting and secondary confinement of the process vessels. In addition, the stainless steel small tank design, with its shorter processing time, minimizes the product stability issues while achieving the desired salt solution decontamination factor (DF). The Team evaluated processing uncertainties related to bounding catalyst activation, foam formation, and TPB recovery, which require additional $R \& D$ demonstration prior to proceeding with detail design. 
A focused economic evaluation regarding moving the precipitate hydrolysis process to the Small Tank TPB Precipitation Facility was conducted since the "High Level Waste Tank Space Management Team Final Report" (Reference 2) recommended a strategy which included placing an evaporator in the DWPF Salt Processing Cell. Based on the study and further technical evaluation, the Team concluded that the precipitate hydrolysis process should be included in the new facility. This approach supports the Tank Farm Salt/Space Management strategy, provides for benzene management in a single-purpose built facility and increased the facility throughput equal to the other alternatives. The net result was that an approximately $\$ 80 \mathrm{M}$ additional capital investment would result in over $\$ 950 \mathrm{M}$ life cycle cost savings.

The Team also recognizes that from a DOE complex perspective, the CST Non-Elutable Ion Exchange alternative exhibits potential benefits. The research conducted on the targeted uncertainties suggest more development of the CST resin is needed to support application with SRS high level salt solution waste. The Team assessed uncertainties related to cesium desorption, resin stability, solids formation, and DWPF interface. The Team believes that these issues can be resolved with the appropriate level of research industry involvement and management attention and result in complex wide savings for technology development. The material stability research would need to be brought to favorable conclusion prior to proceeding with design. The net result on the engineered resolutions was an increase in project costs and life cycle costs. The R\&D effort resulted in an increase in "effective" risk and "effective" uncertainty for project implementation relative to its position at the end of the 1998 Selection Phase.

The Team concluded through the evaluation process that the Direct Disposal in Grout alternative should not be considered. The reasoning for arriving at this decision is primarily the non-technical programmatic risks. Additionally, current R\&D confirms the technology risk associated with MST use. The recommended alternative must have a sure path to operation by 2010 and the closure of the SRS HLW Tanks in accordance with the FFA and STP commitments. The Team knows of no mitigation strategy that would assure that the facility could be commissioned, and that NRC, SCDHEC, and EPA approvals could be obtained, and likely court cases resolved in a manner compatible with this schedule. Although acceptably passing the performance assessment requirements, the Team felt that public acceptance would be more difficult than originally anticipated based on recent interaction with the Citizens Advisory Board. The three sequential risks of regulatory approval, political approval, and judicial approval, all of which have been seen in similar instances, could not be resolved on the necessary schedule with any mitigation strategy the Team could devise. 
It should also be noted that the Team recognized favorable attributes with Caustic Side Solvent Extraction. The limited recent research had positive results, but was not sufficient to change the "effective" risk and "effective" uncertainty regarding project implementation as was concluded in the "HLW Salt Disposition System Engineering Team Final Report" (Reference 7). The relative immaturity of the solvent system was the major deciding factor. Positive attributes associated with this technology were operational, mission and operating schedule flexibility. Additionally, solvent extraction has other development opportunities within the DOE complex and may warrant DOE pursuit of the calixerene science development.

A focused technical and economic evaluation of the current design and plausible alternatives for the removal of uranium, plutonium, neptunium, and strontium from the HLW salt solutions was conducted in accordance with "Position Paper on the Approach to Evaluate Using Existing Facilities for Feed Clarification" (Reference 3). Based on the technical limitations of filtering the resultant material, no viable alternative to the existing approach for feed clarification was identified. The results are documented in the "Alternative for Feed Clarification Study" (Reference 4).

\subsection{Recommended Path Forward}

WSRC recommends that the Small Tank TPB Precipitation be pursued as the most suited technology for SRS high level salt solution waste processing. Investigation should continue into the understanding of catalyst activation and foaming to disposition these key risks. WSRC also recommends that more detailed evaluations and studies for reuse of existing facilities and alternative unit operation technology be performed. R\&D should also continue on the CST Non-Elutable Ion Exchange alternative to address cesium desorption, resin stability, material transport and sampling, and MST filtration risks.

A second option considers the broader DOE complex perspective. This approach would proceed with an aggressive R\&D program solely for the CST Non-Elutable Ion Exchange alternative. The R\&D would focus on cesium desorption, resin stability, and reengineering risks with additional effort to pursue material transport and sampling, MST resuspension and filtration, facility interface issues, and glass qualification. Limited $\mathrm{R} \& D$ efforts to further reduce targeted risk for the Small Tank TPB Precipitation process would continue. 


\subsection{Introduction and Purpose}

The Savannah River Site (SRS) Site Treatment Plan (STP) and Federal Facilities Agreement (FFA) call for closing the HLW Tanks through vitrification of both the long-lived and short-lived radioisotopes in DWPF in preparation for transport to the national high level waste repository. To make this program economically feasible, it is necessary to limit the volume of $\mathrm{HLW}$ glass produced by removing much of the non-radioactive salts and incidental wastes for disposal as saltstone. The ITP facility was designed and constructed to separate the cesium isotopes from the non-radioactive salts so the decontaminated salts could be disposed in a grouted wasteform at the Saltstone facility at SRS.

The ITP process was successfully piloted both on a moderate and full-scale basis with actual SRS waste in the 1980s. During the facility radioactive startup, higher than predicted benzene releases were observed. Additional laboratory and facility tests were initiated to further investigate process chemistry issues. In January 1998, conclusions were drawn from the test program that the benzene release rates associated with facility operation could exceed the capability of the current plant hardware/systems. On January 22, 1998, WSRC informed DOE that ITP chemistry testing demonstrated that the present system configuration could not costeffectively meet the safety and production requirements for the ITP facility and recommended that a study of alternatives to the current system configuration be conducted by a systems engineering team.

On February 6, 1998, the Assistant Secretary for Environmental Management approved a DOESR plan of action to suspend startup-related activities and undertake a systems engineering study of alternatives to ITP. On February 20, 1998, DOE-SR concurred with the WSRC evaluation of the ITP chemistry data, instructed WSRC to suspend ITP startup preparations, and directed WSRC to perform an evaluation of alternatives to the current system configuration for $\mathrm{HLW}$ salt removal, treatment, and disposal.

In March 1998, a WSRC-sponsored High Level Waste Systems Engineering Team was formed to study alternatives to the ITP processes as well as methods to enhance the current process. The multi-disciplined Team was chartered with the task of "systematically developing and recommending an alternative method and/or technology for disposition of HLW salt." The HLW Systems Engineering Team completed the chartered activities, and issued the "HLW Salt Disposition Systems Engineering Team Final Report" (Reference 7) in October 1998.

The U.S. Department of Energy (DOE) - Savannah River Operations Office (SR) completed their review of the WSRC selection process and issued the High Level Waste Salt Disposition Alternatives Evaluation recommendations on January 25, 1999 (Reference 9). The conclusions of their review and a proposed path forward were forwarded to the DOE Assistant Secretary for Environmental Management (EM-1). EM-1 provided authorization to proceed with the DOESR proposed path forward. DOE authorized initiation of a Supplemental Environmental Impact Statement in parallel with performance of additional research on the CST Non-Elutable Ion Exchange and Small Tank TPB Precipitation technologies to address defined technical uncertainties, evaluation of tank farm salt/space management practices, selected trade studies, and additional evaluation of the regulatory/public acceptance for cesium disposal in grout. 


\subsection{Background}

High Level Waste has been produced at the SRS since 1951. This waste was stored in Interim Waste Tanks. In the early 1980s, a concept was developed to no longer construct additional Interim Waste Tanks, but to process the waste into a safer storage form, reduce risk, and ready the waste for permanent storage. This led to an initial design concept for DWPF and an Ion Exchange Facility.

The cost for both facilities was high, and technical uncertainties for Ion Exchange posed too high a risk. Alternatives to the Ion Exchange Process were evaluated and the ITP process was selected due to lower projected cost and technical risk.

The Savannah River Site currently stores 34 million gallons of HLW in Interim Storage Tanks. This activity is considered to be one of the higher risk activities on the Site. The FFA requires removing the waste from the high level waste tanks to resolve several safety and regulatory concerns. Tanks have leaked observable quantities of waste from primary to secondary containment. Other tanks have known penetrations above the liquid level, although no waste has been observed to leak through these penetrations. The "old style" tanks do not meet EPA secondary containment standards for storage of hazardous waste, (effective January 12, 1987). The 34 million gallons of waste stored in the HLW tanks are composed of 31 million gallons of "Salt" and 3 million gallons of sludge. The Sludge process is fully operational. The ITP process was the baseline method intended for handling Salt.

During the facility radioactive startup, higher-than-predicted benzene releases were observed, and a program initiated to investigate process chemistry issues. The program concluded that the benzene release rates associated with facility operation could exceed the capability of the current plant hardware/systems. WSRC informed DOE that the present system configuration could not cost-effectively meet the safety and production requirements for the ITP facility and recommended that a study of alternatives to the current system configuration be conducted by a Systems Engineering team.

With the formation of the Team, a DOE-sponsored charter was issued to guide the systems engineering process for determination of a preferred salt disposition technology. The need for a timely decision was identified. The charter indicated the decision should consider impacts to the following: Limited Tank Farm storage capacity, additional DWPF glass canister production, incurred Life Cycle Cost (LCC) and prolonged environmental risk for liquid waste storage. 


\subsection{High Level Waste System Overview}

Any new salt processing system will interface with existing facilities, and the ease or difficulty of the successful implementation of an alternative technology is governed by how well it will integrate into the existing HLW System.

The HLW System is a set of seven different interconnected processes (Figure 2-1) operated by the High Level Waste and Solid Waste Divisions. These processes function as one large treatment plant that receives, stores, and treats high level wastes at SRS and converts these wastes into forms suitable for final disposal.

These processes currently include:

- High Level Waste Storage and Evaporation (F and H Area Tank Farms)

- Salt Processing (In Tank Precipitation and Late Wash Facilities)

- Sludge Processing (Extended Sludge Processing Facility)

- Vitrification (DWPF)

- Wastewater Treatment (Effluent Treatment Facility)

- Solidification (Saltstone Facility)

- Organic Destruction (Consolidated Incineration Facility)

F and H Area Tank Farm, Extended Sludge Processing, DWPF, Effluent Treatment Facility, Saltstone Facility, and the Consolidated Incineration Facility are all operational. ITP Facility operations are limited to safe storage and transfer of materials. The Late Wash Facility has been tested and is in a dry lay-up status.

The mission of the HLW System is to receive and store SRS high level wastes in a safe and environmentally sound manner and to convert these wastes into forms suitable for final disposal. The planned forms are:

- borosilicate glass to be sent to a Federal Repository

- saltstone to be disposed on site

- treated wastewater to be released to the environment.

Also, the storage tanks and facilities used to process the high level waste must be left in a state such that they can be decommissioned and closed in a cost-effective manner and in accordance with appropriate regulations and regulatory agreements. 
All high level wastes in storage at SRS are Land Disposal Restrictions wastes, which are prohibited from permanent storage. Since the planned processing of these wastes will require considerable time and therefore continued storage of the waste, DOE has entered into a compliance agreement with the EPA and SCDHEC. This compliance agreement is implemented through the STP, which requires processing of all the high level waste at SRS according to a schedule negotiated between the parties.

Figure 2-1 schematically illustrates the routine flow of wastes through the HLW System. The various processes within the system and external processes are shown in rectangles. The numbered streams identified in italics are the interface streams between the various processes. The discussion below represents the HLW System configuration as of January 1998.

Incoming high level wastes are received into HLW Storage and Evaporation ( $\mathrm{F}$ and $\mathrm{H}$ Area Tank Farms) (Stream 1). The function of HLW Storage and Evaporation is to safely concentrate and to store these wastes until downstream processes are available for further processing. The decontaminated liquid from the evaporators are sent to Wastewater Treatment (ETF) (Stream 13).

The insoluble sludges that settle to the bottom of waste receipt tanks in HLW Storage and Evaporation are slurried using hydraulic slurrying techniques and sent to Extended Sludge Processing (ESP) (Stream 2). In ESP, sludges high in aluminum are processed to remove some of the insoluble aluminum compounds. All sludges, including those that have been processed to remove aluminum, are washed with water to reduce their soluble salt content. The spent washwater from this process is sent back to the HLW Storage and Evaporation (Stream 3). The washed sludge is sent to Vitrification (DWPF) for feed pretreatment and vitrification (Stream 4).

Saltcake is redissolved using hydraulic slurrying techniques similar to sludge slurrying. As currently designed, the salt solutions from this operation, and other salt solutions from HLW Storage and Evaporation, were intended for feed to Salt Processing (Stream 5). In ITP, the salt solution would be processed to remove radionuclides, which are concentrated into an organic precipitate. The decontaminated filtrate would then be sent to Tank 50. A concentrated organic precipitate, containing most of the radionuclides, is produced by the process. This precipitate is washed with water to remove soluble salts. However, some soluble corrosion inhibitors that interfere with DWPF processing must be left in the precipitate after washing because the precipitate is stored in carbon steel tanks, which are susceptible to corrosive attack by uninhibited precipitate wastes.

The precipitate is transferred to Late Wash for further washing in stainless steel tanks to reduce the level of soluble corrosion inhibitors to acceptable levels for the DWPF process (Stream 7). The washwater from this process is returned to ITP to be reused in the ITP process (Stream 8). 
The washed precipitate from Late Wash is then sent to the DWPF vitrification building (221-S). In the vitrification building, the precipitate is catalytically decomposed and separated into two streams: a mildly contaminated organic stream and an aqueous stream containing virtually all of the radionuclides. The mildly contaminated organics are stored at DWPF and eventually transferred to Organic Destruction (CIF) (Stream 11). The aqueous stream is combined with the washed sludge from ESP, which has undergone further processing and the mixture vitrified.

The washed sludge from ESP (Stream 4) is chemically adjusted in the DWPF to prepare the sludge for feed to the glass melter. As part of this process, mercury is stripped out, purified, and sent to mercury receivers (Stream 12). The aqueous product from organic decomposition is added to the chemically adjusted sludge. The mixture is then combined with glass frit and sent to the glass melter. The glass melter drives off the water and melts the wastes into a borosilicate glass matrix, which is poured into a canister. The canistered glass wasteform is sent to site interim storage, and will eventually be disposed of in a Federal Repository (Stream 9).

The water vapor driven off from the melter along with other aqueous streams generated throughout the DWPF vitrification building is recycled to HLW Storage and Evaporation for processing (Stream 10).

Overheads from the HLW Storage and Evaporation evaporators are combined with overheads from evaporators in the $\mathrm{F}$ and $\mathrm{H}$ Area Separations processes and other lowlevel streams from various waste generators. This mixture of low-level wastes is sent to the ETF (Stream 13).

In the ETF, these low-level wastes are decontaminated by a series of cleaning processes. The decontaminated water effluent is sent to the $\mathrm{H}$ Area outfall and eventually flows to local creeks and the Savannah River (Stream 14). The contaminants removed from the water are concentrated and sent to Tank 50 (Stream 15).

In Tank 50, the concentrate from the ETF is combined with the decontaminated filtrate from the ITP and sent to Saltstone (Stream 6). In the Saltstone Facility, the liquid waste is combined with cement formers and pumped as a wet grout to a vault (Stream 16). In the vault, the cement formers hydrate and cure, forming a saltstone monolith. The Saltstone Facility vaults will eventually be closed as a landfill 


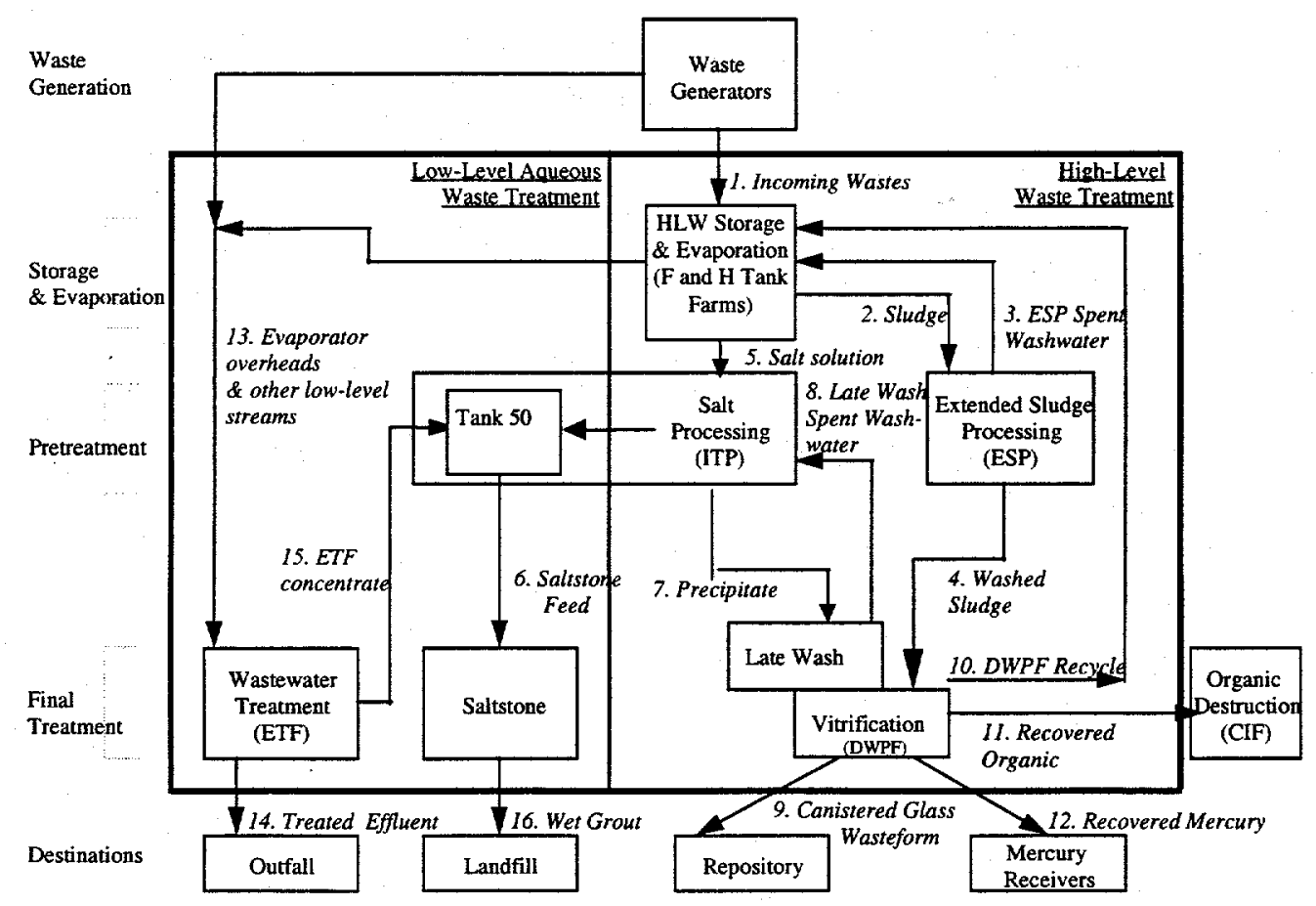

Figure 2-1: HLW System Major Interfaces

\subsection{Team Activities}

Table 2-1 identifies the activities chartered for the Decision Phase. The activities of items 1 through 5 were designated as primary importance to make a technology selection in FY99. Effort on item 6 was to be pursued only as FY99 funding permitted.

Table 2-1: Decision Phase Actions

\begin{tabular}{|l|l|}
\hline $\begin{array}{l}\text { ITEM } \\
\text { NO. }\end{array}$ & DESCRIPTION \\
\hline 1 & $\begin{array}{l}\text { Initiate actions necessary to support the NEPA (Supplemental EIS) process (e.g., evaluate effects on } \\
\text { Saltstone Performance Assessment (PA) from the variations in the feeds from the three alternatives } \\
\text { and provide support as needed to resolve issued identified). }\end{array}$ \\
\hline 2 & $\begin{array}{l}\text { Expand Tank Farm water/salt management studies and develop a strategy and plan to maximize } \\
\text { existing tank space flexibility to accommodate any of the alternatives. }\end{array}$ \\
\hline 3 & $\begin{array}{l}\text { Perform parallel research and development (R\&D) activities to address the technical uncertainties } \\
\text { associated with the Crystaline Silicotitanate (CST) Ion Exchange and Small Tank Tetraphenylborate } \\
\text { (TPB) Precipitation alternatives. }\end{array}$ \\
\hline 4 & $\begin{array}{l}\text { Evaluate the use of existing Tank Farm facilities for the removal of uranium, plutonium, neptunion } \\
\text { and strontium from the HLW salt solutions. }\end{array}$ \\
\hline 5 & $\begin{array}{l}\text { Provide support to DOE-SR as needed for the Direct Disposal in Grout alternative, including any } \\
\text { necessary R\&D activities. }\end{array}$ \\
\hline 6 & Initiate further design development only for issues that are common to all alternatives. \\
\hline
\end{tabular}


The Draft EIS, DOE-EIS-0082-S2D (Reference 1) was subcontracted by DOE to an outside vendor. WSRC has completed the requested tasks in support of the vendor and the Draft EIS is currently scheduled for release in the fourth quarter of calendar year 1999.

WSRC has completed a Systems Engineering evaluation of the tank farm water/salt management approach. The recommended space management strategy and implementation approach are documented in the "High Level Waste Tank Space Management Team Final Report" (Reference 2).

Applied science and technology integration work scope matrix (Reference 8) was developed for CST Non-Elutable Ion Exchange, Small Tank TPB Precipitation, and Direct Disposal in Grout alternatives to identify the key Research and Development (R\&D) items to address technical uncertainties identified in the 1998 Selection Process. The scoped R\&D Experiment List (Table 2-2) was completed (Reference 14) and the detailed results and technology application information used in the Decision Phase process. Under DOE-HQ Efficient Separations Program cross-cut initiative, Oak Ridge and Argonne National Laboratories conducted research and development on Caustic Side Solvent Extraction alternative aimed at addressing technical uncertainties identified in the 1998 Selection Process. This information was also considered during the Decision Phase process. 
Table 2-2: CST Ion Exchange Experiment List

\begin{tabular}{|c|c|c|}
\hline Category & Laboratory Tests & Summary Information \\
\hline $\begin{array}{l}\text { DNFSB 96-1 } \\
\text { "Understanding" Issues }\end{array}$ & $\begin{array}{l}\text { a) Effect of pressure and trace organics } \\
\text { on cesium removal }\end{array}$ & $\begin{array}{l}\text { No effects on resin performance relative to pressure } \\
\text { Pressure and organic resin Kd effects do not appear to affect column } \\
\text { performance } \\
\text { Trace organics confirmed to coat the resin and reduce Kd by } \\
50 \%, \text { but does not effect column performance }\end{array}$ \\
\hline Safety & a) Column gas generation tests & $\begin{array}{l}\text { Gas generation rates equal to calculated amounts } \\
\text { Salt solution turned milky white during static resin test ( } 4 \text { days) } \\
\text { Large scale column tests exhibited sound performance relative to column } \\
\text { hydraulics and gas removal }\end{array}$ \\
\hline Glass Impact & $\begin{array}{l}\text { b) DWPF feed homogeneity from } \\
\text { CSTIX product }\end{array}$ & $\begin{array}{l}\text { Temperature profile for cesium loading testing showed Kd reduction of } \\
90 \% \text { from } 30^{\circ} \mathrm{C} \text { to } 120^{\circ} \mathrm{C} \text {. } \\
\text { The Texas A\&M CST model Kd prediction was higher for all } \\
\text { temperatures vs. actual. } \\
\text { Particle analysis showed binder loss and leaching of silicon from CST } \\
\text { resin. } \\
\text { CST settling rate six times faster than glass frit } \\
\text { As received CST plugged hydraguard sample } \\
\text { Size reduced CST could be sampled, but the sample was not } \\
\text { representative } \\
\text { CST glasses are acceptable for production in DWPF, but will require } \\
\text { new property correlation's to be developed. }\end{array}$ \\
\hline $\begin{array}{l}\text { Operational } \\
\text { Performance }\end{array}$ & $\begin{array}{l}\text { a) Effect of column velocity and } \\
\text { organics on cesium removal } \\
\text { b) Thermal and hydraulic properties } \\
\text { c) Scale column operations }\end{array}$ & $\begin{array}{l}\text { Two side by side column tests } \\
\text { One of the two columns plugged } \\
\text { Simulant post precipitation and resin decoloration observed } \\
\text { Physical properties determined } \\
\text { No post precip until }-3^{\circ} \mathrm{C} \text { which conflicts with understanding } \\
\text { Tall column resin loading and conditioning completed successfully } \\
\text { Tall column hydraulic profile determined and consistent with expectations }\end{array}$ \\
\hline $\begin{array}{l}\text { Confirmation of } \\
\text { Expectations }\end{array}$ & Column tests with Rad Waste & $\begin{array}{l}\text { Saltstone feed specifications met during entire run. } \\
\text { Expected column differential pressure and temperature profiles. } \\
\text { Column plugged during pH adjustment and blockage removed } \\
\text { with backflush }\end{array}$ \\
\hline
\end{tabular}


Table 2-3: Small Tank TPB Precipitation Experiment List

\begin{tabular}{|c|c|c|}
\hline Category & Laboratory Tests & Summary Information \\
\hline Reactor Sizing - Alpha Removal & $\begin{array}{l}\text { a) Simulant tests } \\
\text { b) Real waste tests }\end{array}$ & $\begin{array}{l}\text { MST absorption kinetics supports alpha removal concurrently with } \\
\text { Cesium removal } \\
\text { Consistent results with simulant test }\end{array}$ \\
\hline $\begin{array}{l}\text { Reactor Sizing and Experimental } \\
\text { Conditions for Bench Scale - Cs } \\
\text { Removal }\end{array}$ & $\begin{array}{l}\text { a) } \mathrm{Na} / \mathrm{K} \text { coprecipitation and solubility } \\
\text { b) Cs precipitation and NaTPB } \\
\text { dissolution in CSTR }(0.5 \mathrm{~L})\end{array}$ & $\begin{array}{l}10 \% \text { NaTPB lost to coprecipitation } \\
60 \% \text { excess NaTPB supported } \\
\text { Short circuit test completed ( }<0.1 \%) \\
96 \mathrm{hr} \text { ( } 10 \text { turn-overs) tests run with stable flow/hydraulics and no } \\
\text { major foaming }\end{array}$ \\
\hline Filtration Studies & $\begin{array}{l}\text { a) Produce material for DWPF studies } \\
\text { b) Confirm filtration parameters }\end{array}$ & $\begin{array}{l}\text { Washed precipitate produced } \\
\text { Filter Flux Rates as expected }\end{array}$ \\
\hline $\begin{array}{l}\text { Bench Scale CSTR Studies ( } 20 \\
\text { L) }\end{array}$ & $\begin{array}{l}\text { a) Series CSTR test(s) - open loop } \\
\text { b) Series CSTR test - closed loop with } \\
\text { catalyst and precipitate washing }\end{array}$ & $\begin{array}{l}\text { Short circuit test completed (<0.1\%). } \\
80 \mathrm{hr} \text {. (10 turn-overs) tests (Phase I) run with stable flow/ } \\
\text { hydraulics and a DF }>40,000 \text {. } \\
\text { TPB washing efficiency significantly lower than predicted. } \\
\text { No catalyst activation and no TPB decomposition were observed. } \\
5 \text { concentration cycles and } 4 \text { wash cycles completed. } \\
\text { Nitrite predictions and targets achieved during washing. } \\
230 \text { hr. close coupled test (Phase } 2 \text { ) run with balanced flow/hydraulics. } \\
\text { DF between } 25,000 \text { and } 60,000 \text { obtained. }\end{array}$ \\
\hline DWPF Impacts & $\begin{array}{l}\text { a) Precipitate hydrolysis operation } \\
\text { b) Glass variability study }\end{array}$ & $\begin{array}{l}\text { Process operated as expected within SPC current performance basis. } \\
\text { Higher } \mathrm{Cu}, \mathrm{Ti} \text {, and PHA loading is acceptable }\end{array}$ \\
\hline Confirm Expectations & $\begin{array}{l}\text { a) Real waste test }(0.5 \mathrm{~L}) \text { with catalyst } \\
\text { elements }\end{array}$ & $\begin{array}{l}\text { Foaming problems experienced in CSTRs during Real Waste Test } \\
\text { around the } 76 \mathrm{hr} \text {. run time point } \\
\text { Retest exhibited some foaming and performed in a consistent manner } \\
\text { with demonstration scale system (DF }>100,000 \text { ). }\end{array}$ \\
\hline
\end{tabular}


Table 2-4: Direct Disposal in Grout Experiment List

\begin{tabular}{|c|c|c|}
\hline Category & Laboratory Tests & Summary Information \\
\hline Operational Performance & $\begin{array}{l}\text { a) MTS/Sludge resuspension } \\
\text { b) FlocculentFilter aid }\end{array}$ & $\begin{array}{l}\text { No resuspension problems after } 4 \text { and } 14 \text { day settling period. High } \\
\text { agitator speeds required to resuspend after } 60 \text { days. } \\
\text { Significant viscosity and sheer stress physical property changes } \\
\text { after } 60 \text { days at } 80^{\circ} \mathrm{C} \text {. } \\
\text { No viable and beneficial filter aide identified relative to } \\
\text { improving filter unit flux rates significantly }\end{array}$ \\
\hline Waste Form Impacts & c) Grout performance assessment & Previous performance assessment results confirmed \\
\hline
\end{tabular}

A focused technical and economic evaluation of the current design and plausible alternatives for the removal of uranium, plutonium, neptunium, and strontium from the HLW salt solutions was conducted in accordance with "Position Paper on the Approach to Evaluate Using Existing Facilities for Feed Clarification" (Reference 3). Based on the technical limitations of filtering the resultant material, no viable alternative to the existing approach for feed clarification was identified. The results are documented in "Alternative for Feed Clarification Study" (Reference 4).

The WSRC Management Team, working with key stakeholders in South Carolina and Washington, D.C., has assisted DOE-SR with the advancement of the Direct Disposal in Grout alternative understanding. Additional $R \& D$ was also conducted in the area of grout performance.

Up front planning and design input development has been conducted on those elements common to the technology alternatives in accordance with "Position Paper on Prioritization of Common Systems Applicable to the Recommended and Backup Salt Disposition Technologies" (Reference 11). A Systems Engineering (SE) approach was used for selection of a site location and supporting geotechnical work was conducted. The results are documented in "Site Selection for the Salt Disposition Facility at the Savannah River Site" (Reference 5). Other engineering documents (e.g., Facility Design Description, System Design Description, Statements of Work), have been developed and are under engineering change control.

A Life Cycle Cost (LCC) delta cost analysis was performed to assess the impact of the Consolidated Incineration Facility (CIF) on the four short list alternatives to address an Independent Project Evaluation Team comment. The "Life Cycle Cost Re-Examination (CIF Impacts) Report" (Reference 6) concluded that CIF impact was marginal, and does not provide any cost discrimination between the four alternatives. The disposal cost for organic wastes from Caustic Side Solvent Extraction and Small Tank TPB Precipitation, not considering CIF operation, was determined to be negligible relative to the LCC. 
A focused economic evaluation regarding moving the precipitate hydrolysis process to the Small Tank TPB Precipitation Facility was conducted since the "High Level Waste Tank Space Management Team Final Report" (Reference 2) recommended a strategy which included placing an evaporator in the DWPF salt processing cell. Based on the study and further technical evaluation, it was concluded that the precipitate hydrolysis process should be included in the new facility. This approach supports the Tank Farm Salt/Space Management strategy, provides for benzene management in a single-purpose built facility and increases the facility throughput equal to the other alternatives. The net result was that an approximately $\$ 80 \mathrm{M}$ additional capital investment would result in over \$950M life cycle cost savings.

Submittal of this report completes the FY99 actions assigned to the HLW Salt Disposition Team for the Decision Phase. 


\subsection{Decision Process}

The Decision Phase was structured as a continuation of the HLW Salt Disposition Systems Engineering Team efforts of 1998 (Figure 3-1). The Decision Phase was developed based on the decision logic (Figure 3-2), to address those attributes highlighted as issues in the WSRC Final Report, WSRC-RP-98-0170, DOE-SR Review Team Final Report, letter to James M. Owendoff dated December 16, 1998, and the Independent Project Evaluation Team Review and Assessment Report, DOE/ID-10672. The four short list alternatives were subjected to the Decision Process (Figure 3-3). Science and technology activities were performed to advance understanding for those technical uncertainties which could potentially provide technical discrimination between the alternatives.

\subsection{Process Description of the Four Short List Alternatives}

The conceptual process for each alternative is briefly described below. Key streams for each alternative are described to allow similarities and differences among the alternatives to be compared.

Existing infrastructure in the Tank Farms limits the salt solution removal rate to an average of $6,000,000$ gallons per year at a sodium concentration of $6.44 \mathrm{M}$. This removal rate along with the approximately 80 million gallons of salt solution to be processed serves as the basis for flowsheets and material balances developed for each alternative. Processing rates for each alternative vary up to this maximum based on interface requirements.

For the four alternatives, salt solution is treated with a slurry of solid MST to sorb soluble strontium and alpha-emitting TRU contaminants (U, Pu, Np, Am, Cm). Small Tank TPB Precipitation combines this treatment with simultaneous cesium precipitation. The other three alternatives require separate MST treatment followed by filtration as an initial process step.

Three of the alternatives - Small Tank TPB Precipitation, Caustic-Side Solvent Extraction and CST Non-Elutable Ion Exchange - reduce the cesium concentration to a level that allows continued use of the existing Saltstone Production Facility and vaults located in $\mathrm{Z}$ Area at the Savannah River Site, and continued disposal of salt waste as saltstone in an industrial solid waste landfill. The grout composition for the four alternatives is based on formulations that are comparable to those now used in the current Saltstone Facility.

In the Direct Disposal in Grout alternative, cesium is not removed from the salt solution. Limited shielding in the existing Saltstone Production Facility prevents its use for this alternative. Changes in transfer lines, vaults and permits are also needed to dispose of the saltstone grout. Because of the higher projected cesium concentration, saltstone generated from the Direct Disposal in Grout alternative is within radionuclide concentration limits for Class $\mathrm{C}$ low level waste, as defined by the NRC. 


\subsubsection{Caustic Side Solvent Extraction}

In the proposed Caustic-Side Solvent Extraction alternative, salt solution (6.44 M sodium) is combined with dilution water in the Alpha Sorption Tank (AST) within the new shielded facility. Soluble alpha contaminants and strontium are sorbed on monosodium titanate (MST) solids that are added as a slurry to the salt solution in the AST. The solution is diluted to $\sim 6.1 \mathrm{M}$ sodium in the AST in the combined waste stream.

After confirming that soluble alpha concentration has been reduced to an acceptably low level, the resulting slurry is filtered to remove MST and entrained sludge solids. Clarified filtrate is transferred to the Salt Solution Feed Tank and stored until it can be processed.

After sufficient salt solution has been processed in the AST to yield $\sim 5 \mathrm{wt} \%$ insoluble solids by filtration, MST and sludge solids that have accumulated in the AST are transferred to a Sludge Solids Receipt Tank within the facility, washed to reduce the soluble salt concentration in the accumulated slurry and then stored until the slurry can be transferred to the DWPF and incorporated into HLW glass.

Caustic-Side Solvent Extraction uses a sparingly soluble (in aqueous solution) organic solvent (Isopar L®) containing an organic-soluble extractant (BobCalixC6; also known as calixerene) and aromatic alcohol modifier that complexes cesium nitrate to remove it from clarified salt solution. The Isopar $L \otimes$ solvent contains $0.01 \mathrm{M}$ calixarene and $0.2 \mathrm{M}$ aromatic alcohol modifier and is fed from the Solvent Hold Tank to the Extraction Stages. This organic solution is contacted with a blend of clarified alkaline aqueous waste fed from the Salt Solution Feed Tank and the aqueous phase from the Acid Scrub Stages.

Cesium nitrate (and some potassium nitrate and sodium nitrate) is extracted from the waste into the organic phase, using a series of countercurrent centrifugal contactors (the Extraction Stages). The cations are stabilized in the solvent phase by the calixarene molecule while the nitrate ion is stabilized by the modifier. Due to the size of the opening in the calixarene molecule, cesium is complexed preferentially to sodium and potassium. This selectivity for cesium is more than two orders of magnitude higher than for potassium and more than four orders of magnitude higher than sodium. This higher selectivity for cesium is required to separate cesium effectively from sodium and potassium in the bulk salt solution. The efficiency of cesium separation is further enhanced by contacting the organic phase from the extraction stages with $0.05 \mathrm{M}$ nitric acid using two centrifugal contactors to remove potassium and sodium salts from the solvent stream (the scrub stages).

The organic phase effluent from the scrub stages is next contacted with a very dilute $(0.0005 \mathrm{M})$ nitric acid stream to transfer the cesium to the acidic aqueous stream (the Strip Stages). The aqueous effluent from the strip stages, which is a slightly acidic solution of radioactive cesium nitrate, is sent to an extractant recovery process. 
Aqueous decontaminated salt solution (DSS) from the Extraction Stages will contain a small amount of either soluble or entrained organics. Two additional contactors are used to remove soluble organics and recover calixarene and modifier from the aqueous Raffinate exiting the Extraction Stages (Raffinate Organic Removal Stages). A small amount of Isopar $L \otimes$ is introduced into these stages to facilitate the extraction of the modifier and calixarene from the aqueous phase. The organic phase from these two stages is mixed with recycled organic phase and returned to the Extraction Stages. The DSS from the Raffinate Organic Removal Stages is sent to the Aqueous Raffinate Stilling Tank where any residual entrained organics float to the surface and are decanted. From the Aqueous Raffinate Stilling Tank, DSS is transferred to one of two barium Decay Tanks. These two tanks are sized to allow sufficient hold time for secular equilibrium to be re-established between residual cesium and its decay daughter, barium, before the salt solution is analyzed to determine if it has been adequately decontaminated. After analysis confirms adequate decontamination, the DSS is transferred to one of two DSS Hold Tanks and stored until it can be transferred to $\mathrm{Z}$ Area for processing and disposal as saltstone.

A similar extractant recovery process is also used for the aqueous strip effluent (acidic solution of extracted cesium nitrate). Two additional contactors are used to remove soluble and entrained organics (Strip Organic Removal Stages). As with the extractant recovery from DSS, a small amount of Isopar $L \otimes$ is introduced into these two stages to extract residual modifier and calixarene from the aqueous strip effluent. The organic stream from this operation is returned to the Strip Stages. The aqueous phase is transferred to the Strip Effluent Stilling Tank where any entrained solvent is removed by decanting. The decanted aqueous solution is then sent to the DWPF Salt Feed Tank and stored until it can be transferred to the DWPF for processing into HLW waste glass.

\subsubsection{CST Non-Elutable Ion Exchange}

In the proposed CST Non-Elutable Ion Exchange process, salt solution (6.44 M sodium) is combined with dilution water and spent solutions from filter cleaning in the Alpha Sorption Tank (AST) within the new shielded facility. Soluble alpha contaminants and

${ }^{90} \mathrm{Sr}$ are sorbed on monosodium titanate (MST) solids that are added as a slurry to the salt solution in the AST. The solution is diluted to $\sim 5.6 \mathrm{M}$ sodium in the AST in the combined waste stream that is fed to filtration.

After confirming that soluble alpha concentration has been reduced to an acceptably low level, the resulting slurry is filtered to remove MST and entrained sludge solids that may have accompanied the salt solution to the AST. Clarified filtrate is transferred to the Recycle Blend Tank, where it is combined with other aqueous streams generated from resin loading, pretreatment and unloading operations to prepare the columns for operation. Combining these streams yield $\sim 5.3 \mathrm{M}$ sodium solution. The combined stream is stored until it can be processed through the ion exchange column train loaded with CST. 
The ion exchange train consists of three operating columns in series, identified as lead, middle and guard columns, where the cesium is exchanged onto the CST. A fourth standby column is provided to allow continued operation while cesium -loaded CST is being removed and fresh CST is being added to the previous lead column. The effluent from the guard column is passed through a fines filter to prevent cesium -loaded fines from contaminating the salt solution. The filtered salt solution flows to one of two Product Holdup Tanks and the activity is measured to ensure it meets the saltstone limit for cesium. These two tanks are sized to allow sufficient hold time for secular equilibrium to be re-established between residual cesium and its decay daughter, barium, before the salt solution is analyzed to determine if it has been adequately decontaminated. After analysis confirms adequate decontamination, the DSS is transferred to one of two DSS Hold Tanks and stored until it can be transferred to $Z$ Area for processing and disposal as saltstone.

When the lead column in the train is close to saturation (expected to be $>90 \%$ ), that column is removed from service, the second column becomes the lead column, the third column becomes the middle column, and the fresh, standby column becomes the third, or guard, column. The cesium -loaded CST from the first column is then sluiced with water into one of two Loaded Resin Hold Tanks where it is combined with the fines from the fines filter. Excess sluicing water is removed to produce a $10 \mathrm{wt} \%$ CST slurry in water. The excess water is sent to the Recycle Blend Tank. The CST slurry is stored in the Loaded Resin Hold Tank until it can be transferred to the DWPF for incorporation into HLW waste glass.

Before being loaded into a column, the CST resin must undergo two treatments. First, the CST is loaded into the Column Preparation Tank, similar in dimensions to an ion exchange column bed. The CST is then backflushed with water to remove the fines. These fines are removed by a filter for disposal as industrial waste. The second treatment involves a 24-hour caustic soak. The as-received CST is partially in the hydrogen form and partially in the sodium form. The resin is converted to the sodium form by circulating a sodium hydroxide solution through the Column Preparation Tank for 24 hours. The material is then loaded into an empty standby column by sluicing with water.

After loading the column, sufficient water must be retained in the column to cover the resin bed and exclude air which might cause channeling in the bed. Prior to placing the loaded standby column in service, the water must be displaced by a $2 \mathrm{M}$ sodium hydroxide solution. If this is not done, aluminum may precipitate from the initial salt solution feed as the $\mathrm{pH}$ is reduced by mixing with the residual water. A similar sodium hydroxide flush is required after the a bed is removed from service and before the CST loaded with cesium is sluiced from the bed with water. As noted above, these flushes are sent to the Recycle Blend Tank and combined with clarified salt solution. 


\subsubsection{Direct Disposal in Grout}

At the projected range of concentrations of cesium in salt solution, grout from this process must be produced within a new shielded cell facility, using equipment modified to enable remote operation and maintenance. This facility would be located in $\mathrm{Z}$ Area, near the existing industrial waste landfill now containing vaults used for the disposal of saltstone. Shielded transfer lines and remotely operated valve boxes to direct the grout to different vault cells must be provided. Active ventilation with high-efficiency particulate air filtration (HEPA filtration), rather than the passive ventilation now used, is also needed for the disposal vaults because of the higher cesium concentrations expected.

The salt solution must still be treated to reduce the concentration of soluble TRU contaminants and remove any entrained sludge solids that may be present in the salt solution. This treatment assures the grout is at least within alpha limits for NRC Class C low level waste disposal requirements (100 n curie/g), although the Class A limit for alpha activity ( $10 \mathrm{n}$ curie/g) is preferred for this alternative to facilitate permit modifications for disposal of waste containing higher cesium concentrations. The vault design presently used in the Saltstone Facility meets current regulations for NRC Class C waste disposal. However, the current disposal permit issued by the state of South Carolina presently restricts the average curie content of saltstone placed in a disposal unit (vault cell) to be well within NRC Class A limits.

In the proposed Direct Disposal in Grout alternative, the salt solution (6.44 $\mathrm{M}$ sodium) is transferred to the Alpha Sorption Tank (AST) within the new shielded facility used to produce grout. The solution is first diluted to $\sim 6.0 \mathrm{M}$ sodium using process water and spent wash water from filter cleaning and washing of insoluble solids within the facility. Soluble alpha contaminants and strontium are then sorbed on MST solids that are added as a slurry to the salt solution to reduce their soluble concentrations to levels within NRC Class A limits.

After confirming soluble strontium and alpha concentrations have been reduced to an acceptably low level, the resulting slurry is filtered to remove the MST and any entrained sludge solids in the feed solution. The filtrate is then transferred to a Salt Solution Hold Tank and stored until it can be processed within the facility to produce grout for disposal in a saltstone vault. To assure the product is acceptable for disposal, the clarified salt solution must be diluted to a maximum $\sim 6.0 \mathrm{M}$ sodium concentration or to a cesium concentration that yields a final solid waste product that contains less than 4600 curies of cesium per cubic meter, the regulatory limit for cesium in Class $\mathrm{C}$ low-level waste. Based on the projected feed solution cesium concentrations, cesium concentrations in saltstone from this alternative would average $\sim 240$ curies per cubic meter with a range of concentrations of $\sim 65$ to $\sim 700$ curies per cubic meter., well within the Class $C$ limit.

After concentrating to $\sim 5 \mathrm{wt} \%$ insoluble solids during filtration, MST and sludge solids that collect in the AST are transferred to a Sludge Solids Receipt Tank, washed to reduce the soluble salt concentration in the accumulated slurry and then stored until the slurry can be transferred to the DWPF and incorporated into glass. 


\subsubsection{Small Tank TPB Precipitation}

In the Small Tank TPB Precipitation process, salt solution is received into a Fresh Waste Day Tank located in the new facility. For this continuous precipitation process, salt solution, a solution of sodium tetraphenylborate, a slurry of MST, spent wash water and dilution water are continuously added to a continuous stirred tank reactor (CSTR) located in the new facility. Sufficient dilution water is added to the first CSTR to reduce the sodium molarity to $\sim 4.7 \mathrm{M}$ to optimize conditions for precipitation and MST sorption reactions. The first CSTR feeds a second CSTR to provide the necessary hold up time to complete the reactions. In the CSTRs, soluble cesium and potassium are precipitated as TPB salts and $\mathrm{Sr}, \mathrm{U}, \mathrm{Pu}, \mathrm{Am}, \mathrm{Np}$ and $\mathrm{Cm}$ are sorbed on the MST solids. The resulting slurry, containing $\sim 1 \mathrm{wt} \%$ insoluble solids, is transferred from the second CSTR to the Concentrate Tank from which the slurry is continuously fed to a cross-flow filter to concentrate the solids, which contain most of the radioactive contaminants. Decontaminated salt solution filtrate is transferred to a Filtrate Hold Tank from the filter unit and stored until it can be transferred to the existing Saltstone Production Facility located in $\mathrm{Z}$ Area where it is converted to saltstone for disposal.

After concentrating the slurry to $10 \mathrm{wt} \%$, and accumulating 4,000 to 5,000 gallons in the Concentrate Tank, the slurry is transferred to the Wash Tank and washed to remove soluble sodium salts by adding process water and removing spent wash water by filtration. Spent wash water is either recycled to the first CSTR to provide a portion of the needed dilution water or sent to the Filtrate Hold Tank and on to Z Area where it is converted to saltstone for disposal. At the end of the washing operation, $10 \mathrm{wt} \%$ slurry is transferred to the Precipitate Storage Tank for staging to be processed through the acid hydrolysis unit operation and eventually vitrification. Recovered by-product benzene from acid hydrolysis is transferred to the Consolidated Incinerator Facility (CIF) and incinerated. The aqueous product from acid hydrolysis is combined with sludge feed to the DWPF and incorporated into HLW waste glass.

In the initial proposal for the Small Tank TPB alternative, washed $10 \mathrm{wt} \%$ slurry was to be processed using the existing acid hydrolysis process equipment installed in the DWPF Salt Cell. However, a tank farm salt/space management strategy recommends using the DWPF Salt Cell for location of an acid evaporator. This coupled with the limiting design capacity of the existing acid hydrolysis processing equipment, led to the acid hydrolysis process being moved to the new facility with appropriately sized equipment to support the desired waste removal rate. Moving the acid hydrolysis operation to the new facility offers the advantage of confining the operations involving benzene generation and handling to a single facility, but the footprint of the proposed facility would increase for this alternative. 


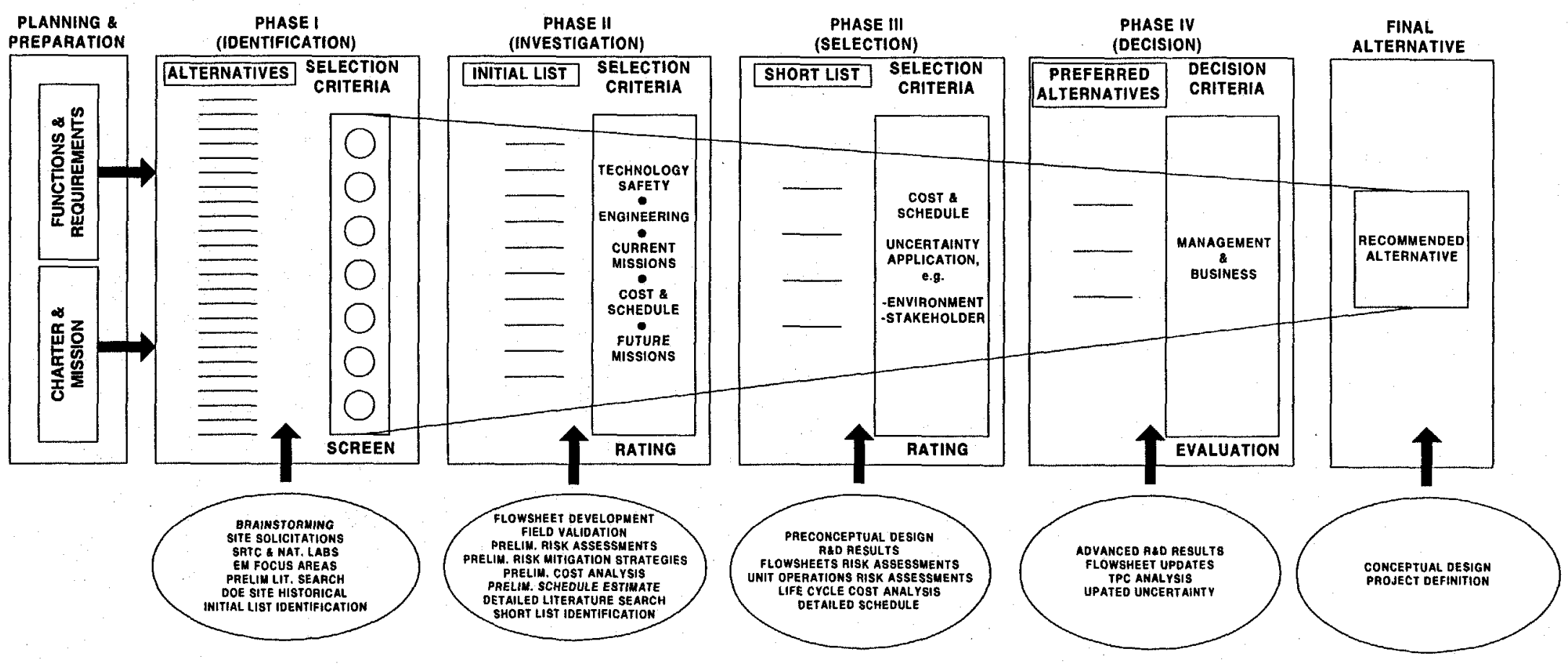

Figure 3-1: Salt Disposition Team Efforts 

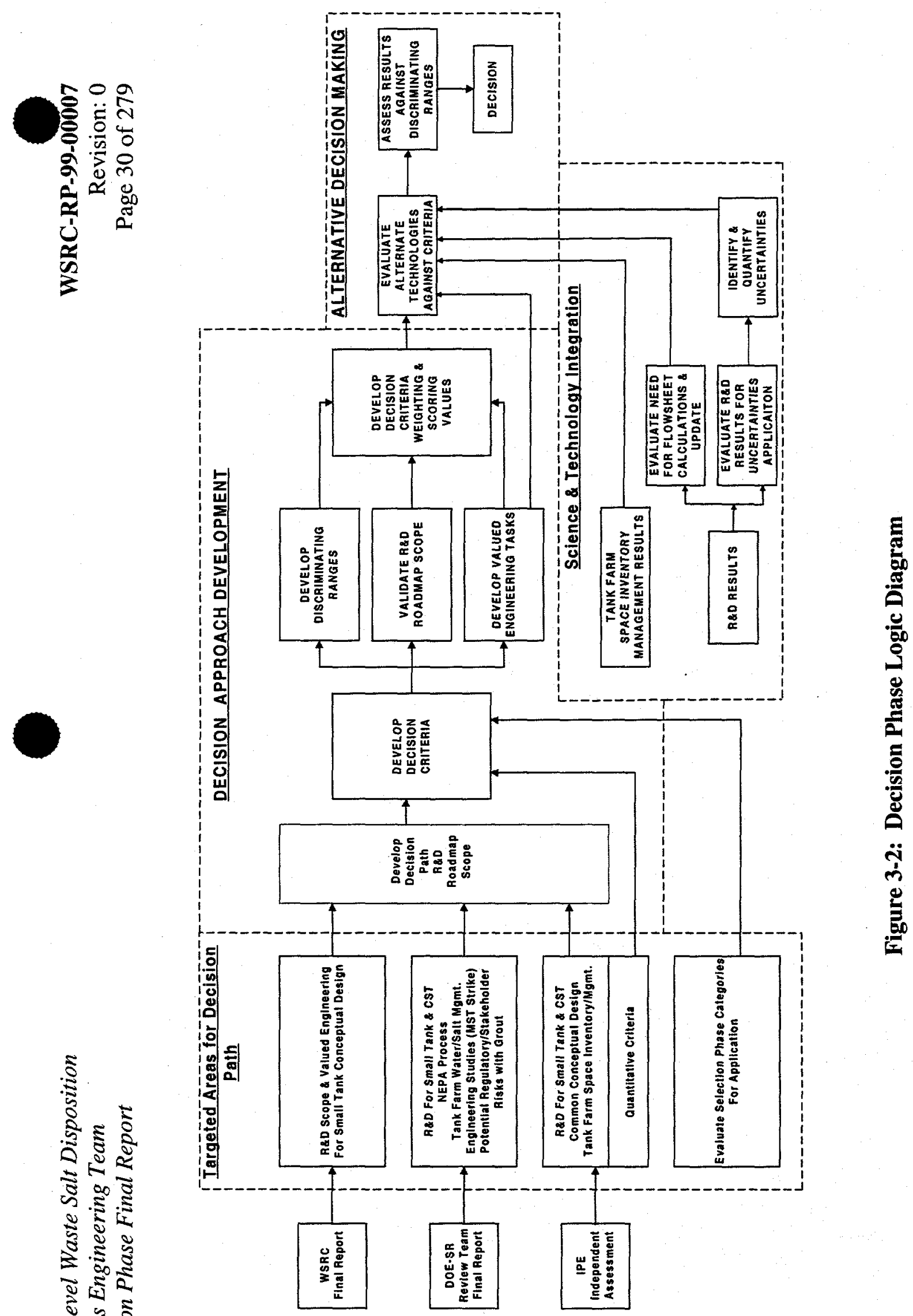


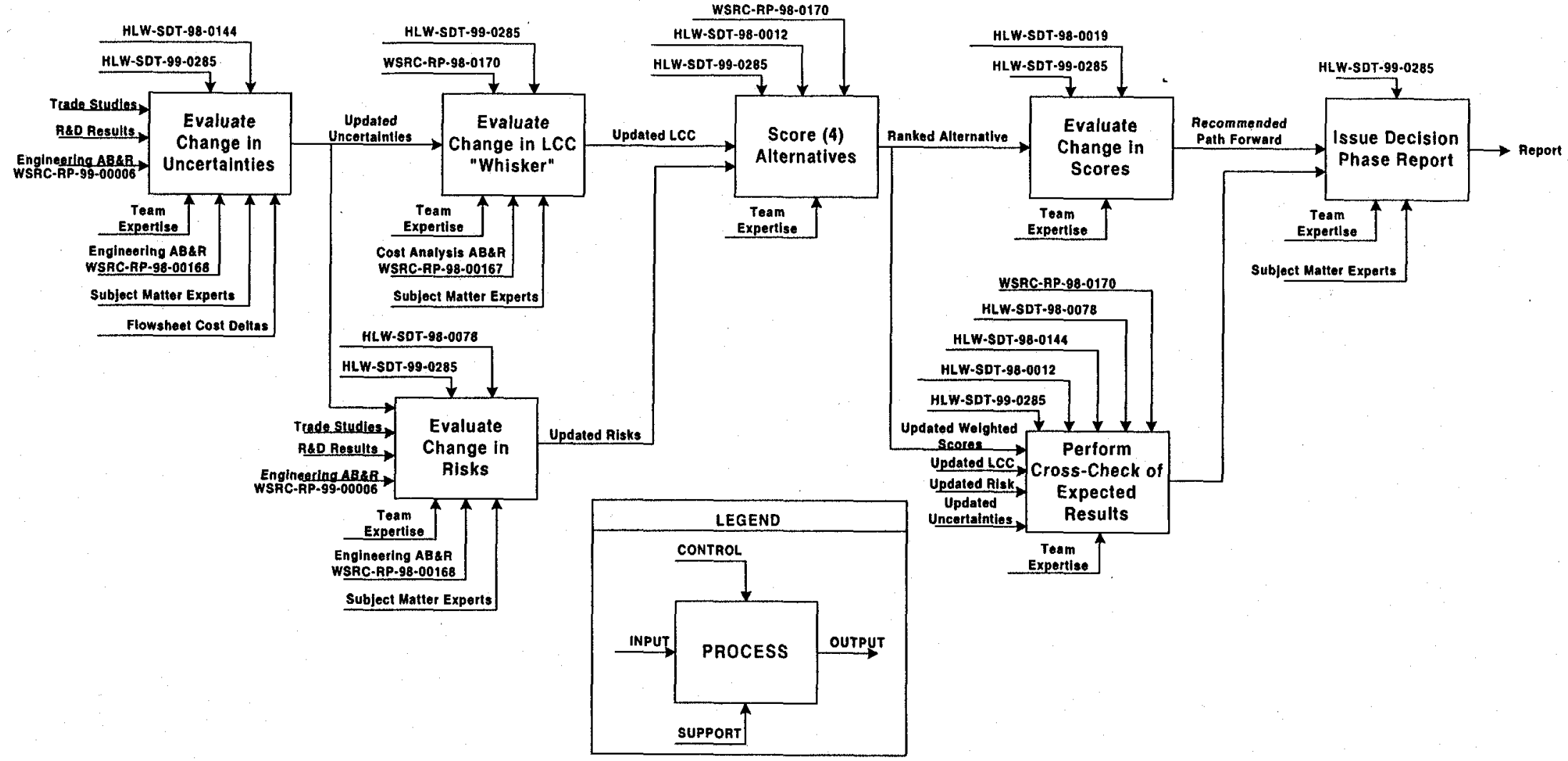

Figure 3-3: Decision Process Business Model 


\subsection{Uncertainties}

The Cost Validation Matrix (Reference 12) is a summary of uncertainties for each alternative evaluated. The process flowsheet, updated $R \& D$ results, and application of the R\&D results to the process flowsheet were reviewed. Based on the latest information, the Team adjusted the uncertainties, uncertainties impact, or added new uncertainties as appropriate. After each alternative was reviewed, a cross-check was performed to ensure each alternative was addressed consistently. Schedule uncertainties are tabulated in Appendix 7.2 for each alternative. The net impact of the schedule uncertainties, relative to the baseline schedule, are illustrated in Figure 3-4.

\subsection{Risks}

The Risk Categorization Matrix (Reference 13) is a summary of potential issues for each alternative evaluated. The process flowsheet, updated R\&D results, and application of the R\&D results to the process flowsheet were reviewed. Based on the latest information, the Team adjusted the risks, risk impact, or added new risks as appropriate. After each alternative was reviewed, a cross-check was performed to ensure each alternative was addressed consistently. If in the process of reviewing the risks, the Team determined an uncertainty was required, then a new uncertainty was added to the Cost Validation Matrix (Appendix 7.3) and addressed accordingly.

\subsection{Life Cycle Costs (LCC)}

A detailed LCC estimate for each alternative was developed during the 1998 Selection Phase (Reference 7). The Team developed a "Box and Whisker" plot (Figure 3-5) to portray the key information on cost, contingency and uncertainty in a pictorial manner. The "point" contained within the shaded box represents the 1998 LCC Point Estimate, including the 50\% probability level contingencies (Reference 7). The "box" represents the upper and lower contingency bounds of the 1998 point estimate. The 1998 dashed "whiskers" represent the net positive or negative uncertainties that are considered to be outside the standard contingency definition.

The assessment of the current understanding resulted in change to both the cost and schedule uncertainties. The solid portion of the "whisker" shows the combined effect.

The Team identified some cost and schedule impacts believed to be confirmed. That is, those uncertainties which shall be realized given today's understanding. The confirmed cost impacts would result in a change to the "point" estimate. The net confirmed cost impact for Caustic Side Solvent Extraction and Direct Disposal in Grout was negligible. The point and whisker to the right of the shaded box shows the net effect of the confirmed uncertainties for CST Non-Elutable Ion Exchange and Small Tank TPB Precipitation. The results of the evaluation are shown on Figure 3-5. 


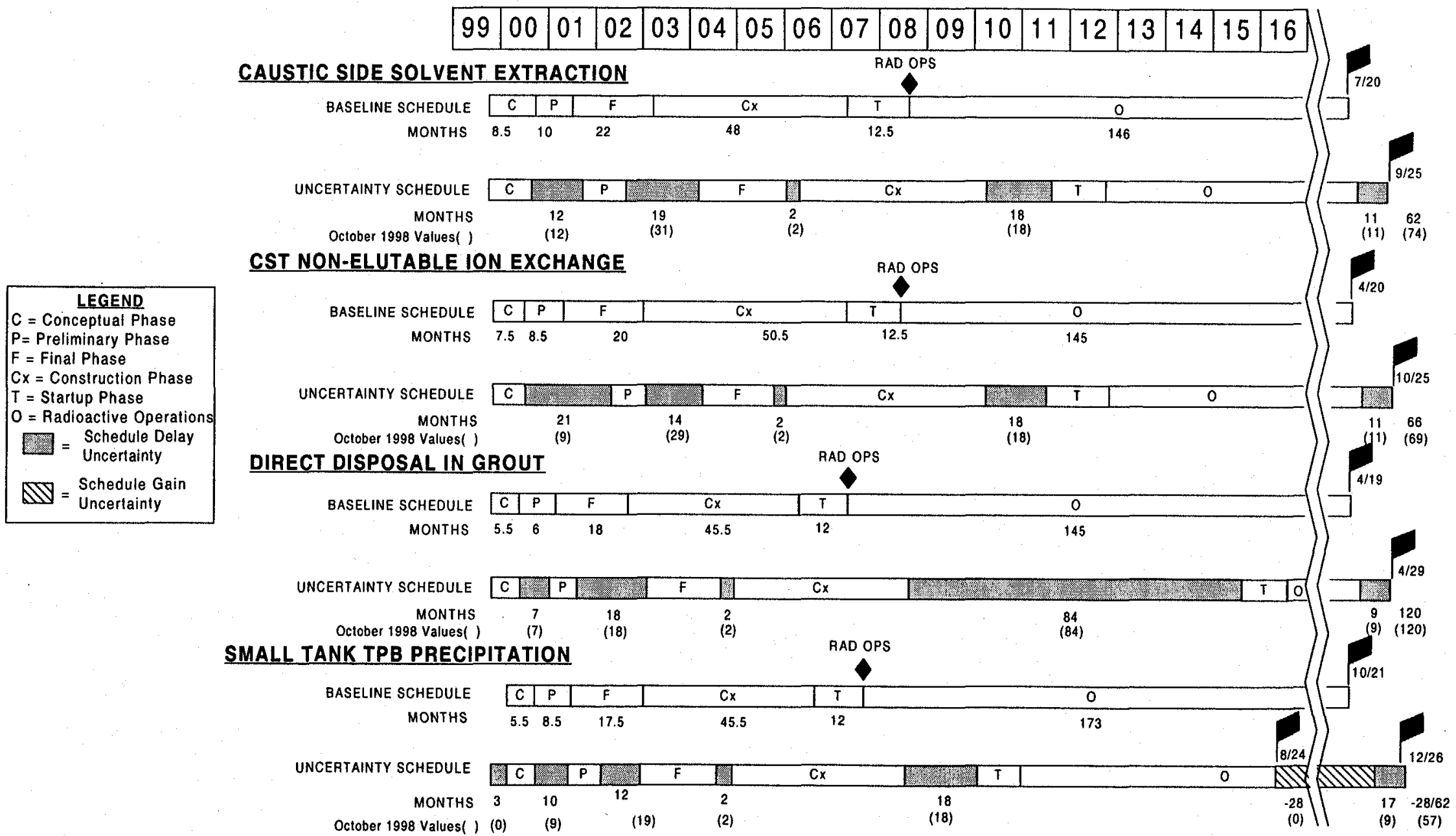

Figure 3-4: Schedule Uncertainties 


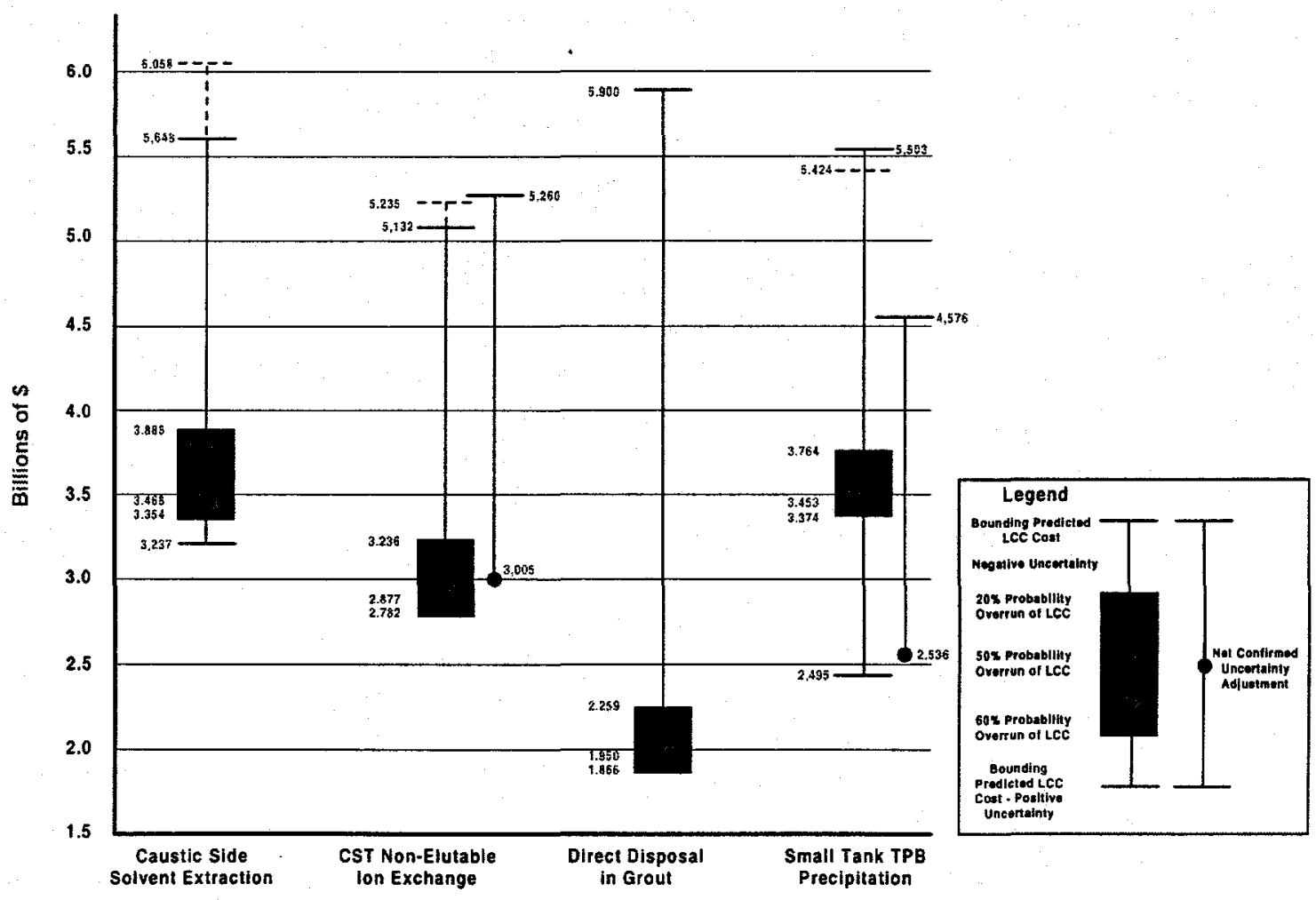

Figure 3-5: Comparative Life Cycle Cost Data

\subsection{Technology Ranking}

The Team scored each alternative against the same Weighted Evaluation Criteria and Utility Function Values (Reference 10) used for the selection of the four short list alternatives. The alternatives were scored in the areas of technology, current missions, future missions, regulatory, engineering, and cost/schedule factoring in the current knowledge of risks, uncertainties, LCC, and process flowsheet. The results for the technology category are displayed in Table 3-1.

\section{Table 3-1: Technology Scoring}

\begin{tabular}{|l|c|c|c|c|}
\hline \multicolumn{1}{|c|}{ Alternative } & $\begin{array}{c}\text { Science } \\
\text { Maturity }\end{array}$ & $\begin{array}{c}\text { Engineering } \\
\text { Maturity }\end{array}$ & $\begin{array}{c}\text { Process } \\
\text { Simplicity }\end{array}$ & Total \\
\hline Caustic Side Solvent Extraction & 4 & 28 & 17 & 49 \\
\hline CST Non-Elutable Ion Exchange & 16 & 28 & 14 & 58 \\
\hline Direct Disposal in Grout & 38 & 28 & 20 & 86 \\
\hline Small Tank TPB Precipitation & 32 & 32 & 14 & 78 \\
\hline
\end{tabular}

- Science Maturity - The level of science understanding needed to minimize project risk.

- Engineering Maturity - The level of applied engineering concepts needed to minimize project risk.

- Process Simplicity - Ease of science implementation understanding by operators. 
Direct Disposal in Grout ranked ahead of the other alternatives on the strength of its science maturity and process simplicity. This is attributable to the basic understanding of mixing dry material with liquid salt solution in a proven formula to make saltstone. Small Tank TPB Precipitation ranked next based on its science maturity. The precipitation process is well understood and has been demonstrated with SRS HLW salt solution waste. CST Non-Elutable Ion Exchange ranked ahead of Caustic Side Solvent Extraction based on its science maturity. The CST resin has been demonstrated with real radioactive waste. However, to be suitable for SRS HLW salt solution waste, the resin manufacturing process would require re-engineering. The solvent solution used in Caustic Side Solvent Extraction has only been demonstrated in a laboratory scale, and thus the lowest score and subsequent rank.

The four alternatives scored relatively the same in engineering maturity. This can be attributed to the wide use of the technologies for different applications throughout the world. Thus, the applied engineering concepts are readily available. This area did not provide any significant discrimination.

\subsection{Cross-Check}

The Team evaluated each alternative to assess the relative change since 1998. The change in the LCC "whisker" was calculated by comparing the 1998 net cost and schedule uncertainty to the 1999 net cost and schedule uncertainty. The weighted score difference was similarly determined. The "effective" risk and "effective" uncertainty was judged by the Team based on the perceived change in being able to successfully deploy the alternative within the baseline project schedule.

The weighted score was the one area where all four alternatives did not reflect the expected result. Additional R\&D was expected to provide higher scores as the understanding and resolution of issues is advanced. Because research in the Decision Phase was targeted at specific uncertainties identified in the Selection Phase, the scores were reduced because of the better understanding of the uncertainties, rather than increased.

Due to the limited research performed for Direct Disposal in Grout and Caustic Side Solvent Extraction, their "effective" risk and "effective" uncertainty were unchanged. The investigation of selected issues with CST Non-Elutable Ion Exchange confirmed the negative issues, and thus increased the "effective" risk and "effective" uncertainty because resolution was not assured within the baseline project schedule. Small Tank TPB Precipitation had confirmation of the issues in a positive manner with resolution being achievable, and a potentially improved operating schedule. This resulted in a reduction of the "effective" risk and "effective" uncertainty. The results are shown in Figure 3-6. 
High Level Waste Salt Disposition

Systems Engineering Team

Final Report
WSRC-RP-99-00007

Revision: 0

Page 37 of 277

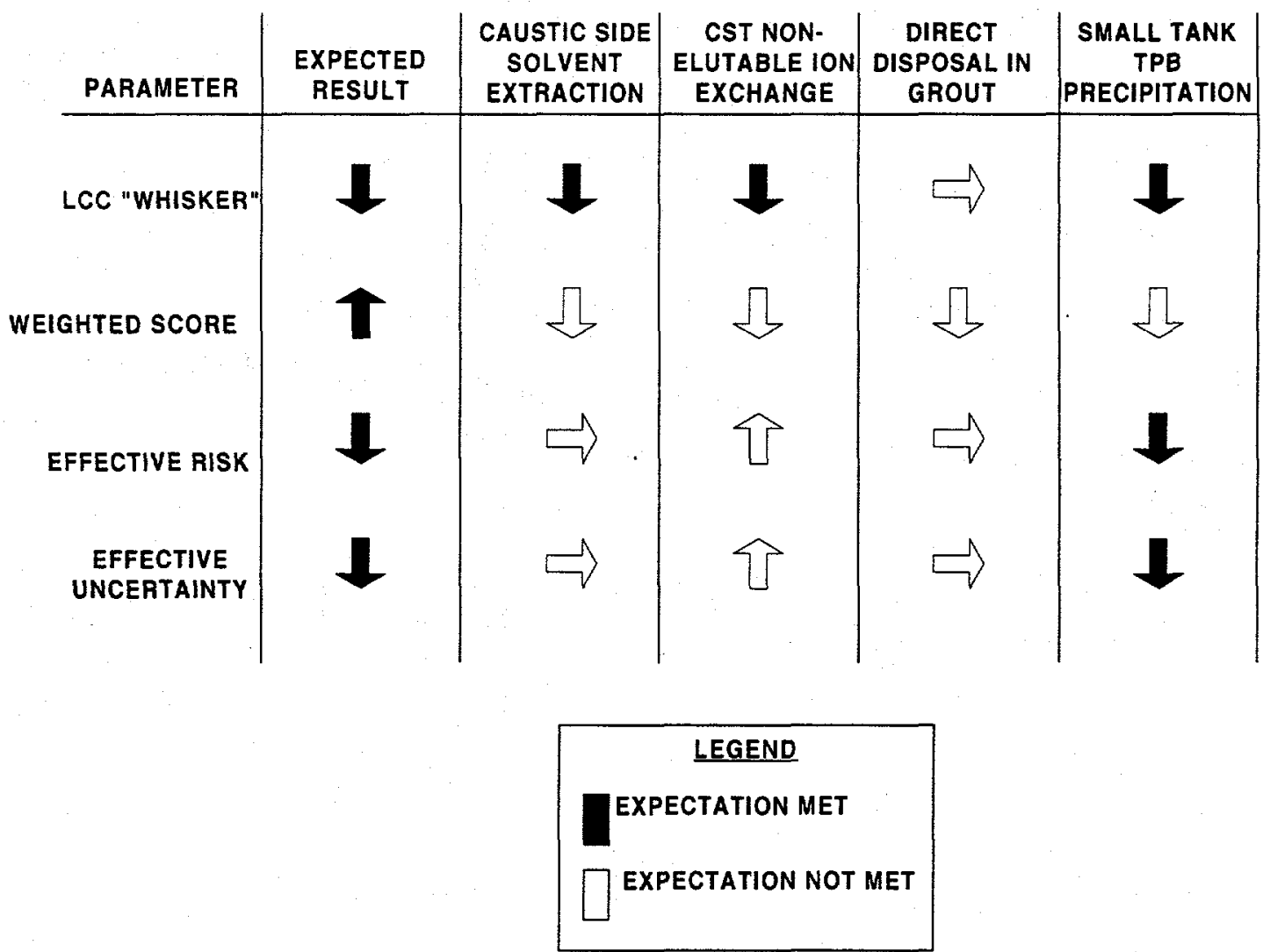

Figure 3-6: Cross-Check Matrix 


\subsection{Results}

The Decision Phase of the Salt Disposition alternative selection process was focused on technical uncertainties identified during the Identification, Investigation, and Selection phases. A detailed science and technology plan was developed and executed to investigate these technical uncertainties. The results of executing the plan and the decision evaluation process have shown that the Small Tank TPB Precipitation alternative is the most suited technical solution relative to current SRS HLW System requirements and needs.

The Decision Phase results also showed that the CST Non-Elutable Ion Exchange alternative is technically sound and identified specific technical items which require further investigation and corrective action. If the remaining open items are addressed, the CST Non-Elutable Ion Exchange alternative can become an effective cesium removal process for the DOE complex.

The Team concluded the most suited technology for processing SRS high level salt solution waste within the defined parameters of Tank Farm Space Management, schedule commitments for decommissioning tanks in the Federal Facilities Agreement and Site Treatment Plan, and interfaces with existing facilities is Small Tank TPB Precipitation. The research conducted has confirmed most of the targeted uncertainties in a positive nature, thus reducing the "effective" risk, and "effective" uncertainties associated with project implementation relative to its position at the end of the 1998 Selection Phase.

\subsection{Small Tank Tetraphenylborate (TPB) Precipitation}

The team recognized that the most significant issue facing the Small Tank TPB Precipitation process is catalytic decomposition of TPB. Safety concerns resulting from TPB decomposition have been addressed in the pre-conceptual design. The process includes positive pressure nitrogen inerting and secondary confinement of the process vessels. The stainless steel design, with its short processing time, minimizes the product stability issues while achieving the desired cesium decontamination factor. To accomplish product decontamination, the pre-conceptual design material balances have assumed TPB decomposition occurs at the highest rate observed during decomposition studies and has conservatively estimated the facility material inventory (residence time). These material balances indicate the desired DF is maintained at the required production capacity.

The Team also recognized that the catalytic activation process leading to decomposition is not completely understood. This is addressed by continued R\&D to better understand these processes and by a feed blending and demonstration strategy for production confirmation. Each $1,000,000$ gallon macro-batch of feed to the facility will be processed by bench scale or larger equipment at process conditions expected to maximize decomposition (the loss of DF). Macro-batches, which do not meet the acceptance criteria will be adjusted by re-blending or a change in process conditions. The Team added schedule uncertainity during the operation time period to accommodate any required rework of the blended salt waste. 
The Team assessed risks in scientific maturity due to the demonstrated need for an antifoam and the inefficient recovery of TPB during the precipitate washing operation. A schedule uncertainty was also applied to the Preliminary Design to ensure antifoam development is completed. The life cycle cost was increased to purchase additional TPB not recovered by the precipitate washing operation.

Additional risk in engineering maturity was based on the need to further define the method of NaTPB introduction into the precipitation reactor (CSTR \#1). Testing to date has demonstrated the importance of NaTPB dispersion to achieve DF.

The Team recognized that the installation of an evaporator in the DWPF Salt Process Cell (SPC), as recommended by the Tank Space Management Team (Reference 2), required the relocation of the precipitate hydrolysis process to this new facility. While this action has an increase in capital cost, it will reduce the operating time by 28 months. This operating time savings is a result of increasing the precipitate hydrolysis process throughput to match the tank farm waste removal limitation and maximizing the DWPF glass production for sludge workoff. Both of these items are corrected to be equivalent to the other alternatives. This is the largest confirmed positive uncertainty for any of the alternatives.

\subsection{CST Non-Elutable Ion Exchange}

The Team recognized the most significant issue with the CST Ion Exchange is stability of the CST in the highly caustic salt solutions. Testing during the year indicates that, following cesium desorption at temperature, re-adsorption by the resin is reduced to essentially zero when precipitation reactions occur. The developer of the product (Texas A\&M University) and the manufacturer of the resin (UOP) have both indicated that this probably occurs from the physical blockage of the resin pores such that cesium cannot diffuse into the resin bead to reach the active resin sites. Precipitation reactions are presumably caused by silica leaching from the resin and subsequent re-precipitation of sodium-aluminum-silicates. The source of the silica is excess material used in the UOP manufacturing process. Both the vendor and developer have indicated that it is possible to eliminate the excess material by re-engineering the manufacturing process, and have further indicated the effort will require from 1 to 2 years, consistent with the original product development. The re-engineered resin must be tested to ensure chemical compatibility and cesium removal efficiency with SRS high level salt solution waste.

Risk associated with scientific maturity was related to the requirement to re-engineer the resin manufacturing process. The risk was previously credited for larger scale radioactive demonstrations (at ORNL), but was adjusted since these were not conducted with highly alkaline waste. Cost and schedule uncertainties were also applied to the Preliminary Design to ensure the re-engineering could be completed. 
Additional risk in engineering maturity was based on the need for large surface area filters (3,000 sq. ft.) and large volume recirculation pumps (6,500 to $8,500 \mathrm{gpm})$ for actinide decontamination due to the low filter flux rate demonstrated during testing. Testing also demonstrated the need to size reduce the particle size of the CST before it can be vitrified in the Defense Waste Processing Facility (DWPF).

The Team identified additional engineered features for management of process temperature, gas disengagement, and explosive gas mixture. The Team also recognized that modifications to the DWPF hydraguard sampling system and re-qualification of the glass product would be required. These items would result in an increase to the project costs and life cycle costs.

\subsection{Direct Disposal in Grout}

The Team concluded through the evaluation process that the Direct Disposal in Grout should not be considered. The reasoning for arriving at this decision is the non-technical programmatic risks. The recommended alternative must have a sure path to operation by 2010 and the closure of the SRS HLW Tanks in accordance with the FFA and STP commitments. The Team knows of no mitigation strategy that would assure that the facility could be commissioned, NRC, SCDHEC, and EPA approvals could be obtained, and likely court cases resolved in a manner compatible with this schedule. Although acceptably passing the performance assessment requirements, the Team felt that public acceptance would be more difficult than originally anticipated based on recent interaction with the Citizens Advisory Board. The three sequential risks of regulatory approval, political approval, and judicial approval, all of which have been seen in similar instances, could not be guaranteed to be resolved on the necessary schedule with any mitigation strategy the Team could devise.

\subsection{Caustic Side Solvent Extraction}

It should also be noted that the Team recognized favorable attributes with Caustic Side Solvent Extraction. The limited recent research had positive results, but was not sufficient to change the "effective" risk and "effective" uncertainty regarding project implementation as was concluded in the 1998 Final Report (Reference 7). The relative immaturity of the solvent system was the major deciding factor. Positive attributes associated with this technology were operational, mission and operating schedule flexibility. However, Team judgement was that solvent extraction would require approximately two years of scientific development. 


\subsection{Recommended Path Forward}

WSRC recommends that the Small Tank TPB Precipitation be pursued as the most suited technology for SRS high level salt solution waste processing. Investigation should continue into the understanding of catalyst activation and foaming to disposition these key risks. WSRC also recommends that more detailed evaluations and studies for reuse of existing facilities and alternative unit operation technology be performed. R\&D should also continue on the CST Non-Elutable Ion Exchange alternative to address cesium desorption, resin stability, material transport and sampling, and MST filtration risks.

A second option considers the broader DOE complex perspective. This approach would proceed with an aggressive $R \& D$ program solely for the CST Non-Elutable Ion Exchange alternative. The R\&D would focus on cesium desorption, resin stability, and resin re-engineering risks, with additional effort to pursue material transport and sampling, MST resuspension and filtration, facility interface issues, and glass qualification. Limited $R \& D$ efforts to further reduce targeted risk for the Small Tank TPB Precipitation process would continue.

WSRC management estimates a potential total project cost savings of $\$ 400 \mathrm{M}$ to $\$ 700 \mathrm{M}$ from the utilization of existing infrastructure at Late Wash, Waste Pretreatment, and Saltstone, and application of alternative unit operation technology. WSRC recommends detailed evaluations and studies be performed to further develop the cost saving concepts. 


\subsection{Acronyms \& Abbreviations}

\begin{tabular}{|c|c|}
\hline $\mathrm{Am}$ & Americium \\
\hline AST & Alpha Sorption Tank \\
\hline CIF & Consolidated Incineration Facility \\
\hline $\mathrm{Cm}$ & Curium \\
\hline Cs & Cesium \\
\hline CST & Crystalline Silicotitanate \\
\hline CSTR & Continuous Stirred Tank Reactor \\
\hline DF & Decontamination Factor \\
\hline DOE & Department of Energy \\
\hline DOE-SR & DOE - Savannah River \\
\hline DSS & Decontaminated Salt Solution \\
\hline DWPF & Defense Waste Processing Facility \\
\hline EPA & Environmental Protection Agency \\
\hline ESP & Extended Sludge Processing \\
\hline ETF & Effluent Treatment Facility \\
\hline FFA & Federal Facilities Agreement \\
\hline HLW & High Level Waste \\
\hline ISMS & Integrated Safety Management System \\
\hline ITP & In Tank Precipitation \\
\hline LCC & Life Cycle Cost \\
\hline MST & Monosodium Titanate \\
\hline $\mathrm{Np}$ & Neptunium \\
\hline NRC & Nuclear Regulatory Commission \\
\hline ORNL & Oak Ridge National Laboratory \\
\hline $\mathrm{Pu}$ & Plutonium \\
\hline $\mathrm{R} \& \mathrm{D}$ & Research and Development \\
\hline SCDHEC & South Carolina Department of Health and Environmental Compliance \\
\hline Sr & Strontium \\
\hline SRS & Savannah River Site \\
\hline SRTC & Savannah River Technology Center \\
\hline STP & Site Treatment Plan \\
\hline TPB & Tetraphenylborate \\
\hline TRU & Transuranic \\
\hline $\mathrm{U}$ & Uranium \\
\hline WSRC & Westinghouse Savannah River Company \\
\hline
\end{tabular}




\subsection{References}

1. DOE-EIS-0082-S2D, "Draft Supplemental Environmental Impact Statement, HLW Salt Disposition Alternatives at Savannah River Site".

2. WSRC-RP-99-00005, "High Level Waste Tank Space Management Team Final Report".

3. HLW-SDT-99-0081, "Position Paper on the Approach to Evaluate Using Existing Facilities for Feed Clarification".

4. HLW-SDT-99-0289, "Alternative for Feed Clarification Study".

5. WSRC-RP-99-00513, "Site Selection for the Salt Disposition Facility at the Savannah River Site".

6. HLW-SDT-99-0306, "Life Cycle Cost Re-Examination (CIF Impacts)".

7. WSRC-RP-98-00170, "HLW Salt Disposition System Engineering Team Final Report".

8. HLW-SDT-99-0009, “Applied Technology Integration Scope of Work Matrix for Decision Making (Small Tank TPB Precipitation, CST Non-Elutable Ion Exchange, and Direct Disposal in Grout".

9. Roy J. Schepens to Austin B. Scott letter dated January 28, 1999, "Additional Evaluation of Salt Disposition Alternatives".

10. HLW-SDT-980006, "Position Paper on the Use of Weighted Evaluation Criteria to Select the Short List of Alternatives".

11. HLW-SDT-99-0018, "Position Paper on Prioritization of Common Systems Applicable to the Recommended and Backup Salt Disposition Technologies”.

12. HLW-SDT-98-0144, "High Level Waste Salt Disposition Application of Uncertainty Document"

13. HLW-SDT-98-0078, "Results Report on Determination of Risk"

14. HLW-SDT-99-0270, "Decision Phase Research and Development Compendium"

15. Milt Levensen and Greg Choppin to Ernest J. Moniz letter dated October 14, 1999, "National Research Council Committee Alternative Processing Options Interim Report" 
High Level Waste Salt Disposition

Systems Engineering Team

Final Report

8.0 Appendices
WSRC-RP-99-00007

Revision: 0

Page 44 of 277 
High Level Waste Salt Disposition

Systems Engineering Team

Final Report
WSRC-RP-99-00007

Revision: 0

Page 45 of 277

8.1 Weighted Evaluation Criteria and Utility Function Value Forms 


\section{HLW Salt Disposition Systems Engineering Team} Level 1 Evaluation Criteria Assessment Form

Alternative Number: 14 Alternative Title Caustic Side Solvent Extraction

Date: $9 / 18 / 99$

A. Evaluation Criterion Technology

Title:

Evaluation Criterion Description: will result in adequate attainment.

B. Evaluation Criterion ID \#: (Note 1)

1.0

C. Evaluation Criterion Weighted

$\mathbf{W}_{1}=$ .23

Value:

D. Utility Functions:

Utility Function (UF) Value $\left(\mathrm{V}_{1}\right)=\Sigma$ Level 2 Criterion Weighted Score (WS)

(Note 2)

E. UF Value Formula: $\quad \mathbf{V}_{1}=\mathbf{W S}_{1.1}+\mathbf{W S}_{1.2}+\mathbf{W S}_{1}$ (Note 3)

F. Evaluation Criterion weighted score for the Alternative: $\quad W_{1} \times V_{1}=$ Weighted Score $\therefore . .23 \times \underline{49.00}=$ Explanatory Notes for Weighted Score:

Notes:

1. For Level 1 Evaluation Criterion, the ID\# is described by X.0, where $X=1,2,3$,etc. For Level 2 Evaluation Criterion, the ID\# is described by X.1, X.2, X.3, etc. where ' $X$ ' is the Level 1 Evaluation Criterion ID\#.

2. Utility Function values range from 0 (least desirable) to 100 (most desirable).

3. If Level 2 Criterion are used, the sum of the Level 2 "Weighted Scores" must be multiplied by the Level 1 Weight to determine the Level 1 Weighted Score. 


\section{HLW Salt Disposition Systems Engineering Team} Level 2 Evaluation Criteria Assessment Form

Alternative Number:__ 14 Alternative Title Caustic Side Solvent Extraction

Date: $9 / 18 / 99$

A. Evaluation Criterion

Scientific Maturity

Title:

Evaluation Criterion

Description:

The level of scientific understanding needed to minimize project risk.

B. Evaluation Criterion $\mathrm{ID}$ \#: (Note 1)

C. Evaluation Criterion Weighted Value:

$\mathbf{W}_{2}=$ .4

D. Utility Functions:

UF Value

(Note 2)

Utility Function (UF) Description:

UF.1 Reliable radioactive production scale demonstration \& correlation to predicted scientific results.

JF.2 Large scale radioactive test; 'spiked' radiochemistry demonstration.

UF.3 Pilot (small) scale radioactive test; full radiochemistry. $\underline{40}$

UF.4 Lab scale test; simulant/real waste. $\underline{10}$

UF.5 Theoretical understanding only; no practical demonstration.
E.

UF VALUE:

Explanatory Notes for UF Selected:

Cesium batch extractant/stripping with real Hanford and SRS waste. Lab scale testing for this solvent. Demo for cesium separation with alkaline solution in two centimeter contractors. (Peterson Summary Phase III)

F. Evaluation Criterion weighted score (WS) for the Alternative:

$$
\mathrm{W}_{2} \times \mathrm{V}_{2}=\mathrm{WS} \therefore \underline{.4} \times \underline{10}=
$$

Notes:

1. For Level 1 Evaluation Criterion, the ID\# is described by X.0, where $X=1,2,3$,etc. For Level 2 Evaluation Criterion, the ID\# is described by X.1, X.2, X.3, etc. where ' $X$ ' is the Level 1 Evaluation Criterion ID\#.

2. Utility Function values range from 0 (least desirable) to 100 (most desirable).

3. If Level 2 Criterion are used, the sum of the Level 2 "Weighted Scores" must be multiplied by the Level 1 Weight to determine the Level 1 Weighted Score. 


\section{HLW Salt Disposition Systems Engineering Team} Level 2 Evaluation Criteria Assessment Form

Alternative Number: 14 Alternative Title Caustic Side Solvent Extraction

Date: 9/18/99

A. Evaluation Criterion Engineering Maturity Title:

Evaluation Criterion The level of applied engineering concepts needed to minimize project risk.

Description:

B. Evaluation Criterion ID \#: (Note 1)

C. Evaluation Criterion Weighted

$\mathbf{W}_{2}=$ .4 Value:

D. Utility Functions:

UF Value

(Note 2)

Utility Function (UF) Description:

UF.1 Reliable radioactive production scale with significant operating experience.

100

UF.2 Reliable non-radioactive production scale with significant operating experience.

$\underline{60}$

UF.3 Limited radioactive production scale. $\underline{40}$

UF.4 Limited non-radioactive production scale $\underline{20}$

UF.5 Demonstration $\underline{0}$
E.

UF VALUE:

Explanatory Notes for UF Selected:
$\mathbf{V}_{2}=$ 70

SRS (centrifugal contactors). France (centrifugal contactactors), Sellafield and Russia experience with radioactive operation with solvent extraction. Historically, the preferred method for chemical separation. Alpha removal process provides some engineering challenges in the areas of filtration, mixing, and pumping. WSRC-TR-00342; WSRC-RP99-006

F. Evaluation Criterion weighted score (WS) for the Alternative:

$\mathrm{W}_{2} \times \mathrm{V}_{2}=\mathrm{WS} \therefore . \mathbf{. 4} \times \underline{70}=$

28.00

Notes:

1. For Level 1 Evaluation Criterion, the ID\# is described by X.0, where $X=1,2,3$,etc. For Level 2 Evaluation Criterion, the ID\# is described by X.I, X.2, X.3, etc. where ' $X$ ' is the Level 1 Evaluation Criterion ID\#.

2. Utility Function values range from 0 (least desirable) to 100 (most desirable).

3. If Level 2 Criterion are used, the sum of the Level 2 "Weighted Scores" must be multiplied by the Level 1 Weight to determine the Level 1 Weighted Score. 


\section{HLW Salt Disposition Systems Engineering Team Level 2 Evaluation Criteria Assessment Form}

Alternative Number:_14 Alternative Title Caustic Side Solvent Extraction

Date: $9 / 18 / 99$

A. Evaluation Criterion Process Simplicity

Title:

Evaluation Criterion Ease of Science implementation understanding by operators.

Description:

B. Evaluation Criterion ID \#:

(Note 1)

C. Evaluation Criterion Weighted

$\mathbf{W}_{2}=$ .2

Value:

D. Utility Functions:

UF Value

(Note 2)

Utility Function (UF) Description:

UF.1 Low complexity, straight forward operations.

UF.2 Moderate complexity - operator aids and routine engineering support.

UF.3 Complex - significant training for operators and continuous, specialized engineering support required.

E.

UF VALUE:

$\mathbf{V}_{2}=$ 85

Explanatory Notes for UF Selected:

Limited unit operations. Successful canyon experience with centrifugal contactor operations.

F. Evaluation Criterion weighted score (WS) for the Alternative:

$\mathrm{W}_{2} \times \mathrm{V}_{2}=\mathrm{WS} \therefore .2 \times \underline{\mathbf{8 5}}=$ 17.00

Notes:

1. For Level 1 Evaluation Criterion, the ID\# is described by X.0, where $X=1,2,3$,etc. For Level 2 Evaluation Criterion, the ID\# is described by X.1, X.2, X.3, etc. where ' $X$ ' is the Level 1 Evaluation Criterion ID\#.

2. Utility Function values range from 0 (least desirable) to 100 (most desirable).

3. If Level 2 Criterion are used, the sum of the Level 2 "Weighted Scores" must be multiplied by the Level 1 Weight to determine the Level 1 Weighted Score. 
HLW Salt Disposition Systems Engineering Team

Level 1 Evaluation Criteria Assessment Form

Alternative Number: 14 Alternative Title Caustic Side Solvent Extraction

Date: $9 / 18 / 999 / 18 / 99$

A. Evaluation Criterion Current Mission Interfaces Title:

Evaluation Criterion Impact on current SRS missions/programs.

Description:

B. Evaluation Criterion ID \#: (Note 1)

C. Evaluation Criterion Weighted

$W_{1}=$ .15

Value:

D. Utility Functions:

Utility Function (UF) Value $\left(V_{1}\right)=\Sigma$ Level 2 Criterion Weighted Score (WS)

(Note 2)

UF Value Formula: $\quad \mathbf{V}_{1}=\mathbf{W S}_{2.1}+\mathbf{W S}_{2.2}+\mathbf{W S}_{2.3}+\mathbf{W S}_{2.4}+\mathbf{W S _ { 2 . 5 }}$ (Note 3)

F. Evaluation Criterion weighted score for the Alternative: $\quad W_{1} \times V_{1}=$ Weighted Score $\therefore .15 \times \underline{66.25}=$ Explanatory Notes for Weighted Score:

Notes:

1. For Level 1 Evaluation Criterion, the ID\# is described by $X .0$, where $X=1,2$, 3, etc. For Level 2 Evaluation Criterion, the ID\# is described by $X .1, X .2, X .3$, etc. where ' $X$ ' is the Level 1 Evaluation Criterion ID\#.

2. Utility Function values range from 0 (least desirable) to 100 (most desirable).

3. If Level 2 Criterion are used, the sum of the Level 2 "Weighted Scores" must be multiplied by the Level 1 Weight to determine the Level 1 Weighted Score. 


\section{HLW Salt Disposition Systems Engineering Team Level 2 Evaluation Criteria Assessment Form}

Alternative Number: 14 Alternative Title Caustic Side Solvent Extraction

Date: $9 / 18 / 99$

A. Evaluation Criterion Title:

Evaluation Criterion Description:

DWPF

Impact on DWPF (Table 1 Functions \& Requirements).

B. Evaluation Criterion ID \#: (Note 1)

C. Evaluation Criterion Weighted

$\mathbf{W}_{2}=$ .25 Value:

D. Utility Functions:

UF Value

(Note 2)

Utility Function (UF) Description:

\section{Sludge only to completion}

UF.2 Sludge plus MST to completion.

$\underline{85}$

UF.3 Baseline - current ITP flowsheet.

$\underline{70}$

UF.4 Moderate impact - some additional canisters (<50\%). Facility modifications required.

$\underline{20}$

UF.5 Significant impact - additional canisters (>50\%) glass reformulation/repermitting required. Major facility modifications required.

E.

UF VALUE:

$\mathbf{V}_{2}=$ 85

Explanatory Notes for UF Selected: No Salt Processing Cell operation. Reduced nitric acid addition by DWPF as trim chemicals. The product stream provides a soft interface with DWPF.

F. Evaluation Criterion weighted score (WS) for the Alternative:

$\mathbf{W}_{2} \times \mathbf{V}_{2}=\mathbf{W S} \therefore$ $\underline{.25} \times \underline{85}=$ 21.25

Notes:

1. For Level 1 Evaluation Criterion, the ID\# is described by $X .0$, where $X=1,2,3$, etc. For Level 2 Evaluation Criterion, the ID\# is described by X.1,X.2, X.3, etc. where ' $X$ ' is the Level 1 Evaluation Criterion ID\#.

2. Utility Function values range from 0 (least desirable) to 100 (most desirable).

3. If Level 2 Criterion are used, the sum of the Level 2 "Weighted Scores" must be multiplied by the Level 1 Weight to determine the Level 1 Weighted Score. 


\section{HLW Salt Disposition Systems Engineering Team} Level 2 Evaluation Criteria Assessment Form

Alternative Number: 14 Alternative Title Caustic Side Solvent Extraction

Date: $9 / 18 / 99$

A. Evaluation Criterion

Title:

Evaluation Criterion

Description:

Saltstone

Impact on Saltstone (Table 1 Functions \& Requirements)

B. Evaluation Criterion $\mathrm{ID}$ \#: $\quad 2.2$ (Note 1)

C. Evaluation Criterion Weighted

$\mathbf{W}_{2}=$ .15 Value:

D. Utility Functions:

UF Value

(Note 2)

Utility Function (UF) Description:

UF.1 No need for Saltstone Facility.

UF.2 Reduced throughput required to Saltstone Facility. No hazards release (Benzene).

$\underline{80}$

UF.3 180M gallons saltstone plus Benzene risk (current flowsheet).

$\underline{70}$

UF.4 Moderate increase in saltstone $(<50 \%)$. Minor facility modifications.

$\underline{40}$

UF.5 Repermit saltstone to Class $\mathrm{C}$ waste. Major facility modifications and increased throughtput $(>50 \%)$. $\quad \underline{0}$

E.

UF VALUE:

$\mathbf{V}_{2}=$ 80

Explanatory Notes for UF Selected:

Reduction in saltstone production by 30 million gallons of saltstone grout. No benzene release. Low solvent solubility (20 ppm in aqueous). WSRC-RP-99-0006 for alpha removal.

F. Evaluation Criterion weighted score (WS) for the Alternative:

$\mathrm{W}_{2} \times \mathrm{V}_{2}=\mathrm{WS} \therefore \underline{.15} \times \underline{80}=$ 12.00

Notes:

1. For Level I Evaluation Criterion, the ID\# is described by X.O, where $X=1,2,3$,etc. For Level 2 Evaluation Criterion, the ID\# is described by X.1, X.2, X.3, etc. where ' $X$ ' is the Level 1 Evaluation Criterion ID\#.

2. Utility Function values range from 0 (least desirable) to 100 (most desirable).

3. If Level 2 Criterion are used, the sum of the Level 2 "Weighted Scores" must be multiplied by the Level 1 Weight to determine the Level I Weighted Score. 
HLW Salt Disposition Systems Engineering Team

Level 2 Evaluation Criteria Assessment Form

Alternative Number: 14 Alternative Title Caustic Side Solvent Extraction

Date: $9 / 18 / 99$

A. Evaluation Criterion

Title:

Evaluation Criterion

Description:

Solid Waste

Impact on Solid Waste (Table 1 Functions \& Requirements).

B. Evaluation Criterion ID \#: (Note 1)

$\underline{2.3}$

C. Evaluation Criterion Weighted

$\mathbf{W}_{2}=$ .1

Value:

D. Utility Functions:

UF Value

(Note 2)

Utility Function (UF) Description: $\underline{100}$

$\underline{80}$

$\underline{50}$

$\underline{30}$

$\underline{0}$

E.

UF VALUE:

Explanatory Notes for UF Selected:
Reduction in liquid benzene generation by 35,000 gallons per year (no benzene generated).

F. Evaluation Criterion weighted score (WS) for the Alternative: $\quad \mathbf{W}_{2} \times \mathbf{V}_{2}=\mathbf{W S} \therefore . .1 \times \underline{80}=$ 8.00

Notes:

1. For Level 1 Evaluation Criterion, the ID\# is described by X.O, where $X=1,2,3$,etc. For Level 2 Evaluation Criterion, the ID\# is described by X.1, X.2, X.3, etc. where ' $X$ ' is the Level 1 Evaluation Criterion ID\#.

2. Utility Function values range from 0 (least desirable) to 100 (most desirable).

3. If Level 2 Criterion are used, the sum of the Level 2 "Weighted Scores" must be multiplied by the Level 1 Weight to determine the Level 1 Weighted Score. 
HLW Salt Disposition Systems Engineering Team Level 2 Evaluation Criteria Assessment Form

Alternative Number: 14 Alternative Title Caustic Side Solvent Extraction

Date: $9 / 18 / 99$

A. Evaluation Criterion Tank Farm

Title:

Evaluation Criterion Impact on Tank Farm (Table 1 Functions \& Requirements).

Description:

B. Evaluation Criterion ID \#: (Note 1)

2.4

C. Evaluation Criterion Weighted

$\mathrm{W}_{2}=$ .2 Value:

D. Utility Functions:

UF Value

(Note 2)

Utility Function (UF) Description: $\underline{100}$

$\underline{50}$ impact.

UF.2 Current flowsheet.
E.

UF VALUE:

Explanatory Notes for UF Selected:
Solvent extraction concentration factor is designed to be the same as the current flowsheet WSRCC-RP-98-0168, R1

F. Evaluation Criterion weighted score (WS) for the Alternative:

$$
\mathrm{W}_{2} \times \mathrm{V}_{2}=\mathrm{WS} \therefore \underline{.2} \times \underline{50}=
$$

Notes:

1. For Level 1 Evaluation Criterion, the ID\# is described by X.0, where $X=1,2,3$,etc. For Level 2 Evaluation Criterion, the ID\# is described by X.I, X.2, X.3, etc. where ' $X$ ' is the Level 1 Evaluation Criterion ID\#.

2. Utility Function values range from 0 (least desirable) to 100 (most desirable).

3. If Level 2 Criterion are used, the sum of the Level 2 "Weighted Scores" must be multiplied by the Level 1 Weight to determine the Level 1 Weighted Score. 


\section{HLW Salt Disposition Systems Engineering Team} Level 2 Evaluation Criteria Assessment Form

Alternative Number: 14 Alternative Title Caustic Side Solvent Extraction

Date: $9 / 18 / 99$

A. Evaluation Criterion Title:

Evaluation Criterion Description:

Tank Farm Space Management.

Utilization of available Tank Farm storage \& resources as a function of time (HLW Salt Disposition Interface Functional Performance Requirement).

B. Evaluation Criterion ID \#: (Note 1)

C. Evaluation Criterion Weighted Value:

$\mathrm{W}_{2}=$ .3

D. Utility Functions:

UF Value

(Note 2)

Utility Function (UF) Description:

Accelerate tank space gain. Tank space adequate for current and future missions.

$\underline{100}$

UF.1

Current flowsheet (reduces available tank space)

$\underline{40}$

UF.3 Accelerated reduction in available tank space (water logged tank farm).

E.

\section{UF VALUE:}

Explanatory Notes for UF Selected:

$$
\mathbf{V}_{2}=
$$
50 completed. WSRC-RP-98-00168, R1; WSRC-RP-99-0005.

F. Evaluation Criterion weighted score (WS) for the Alternative:

$\mathbf{W}_{2} \times \mathbf{V}_{2}=\mathbf{W S} \therefore \underline{.3} \times \underline{\mathbf{5 0}}=$

15.00

Notes:

1. For Level 1 Evaluation Criterion, the ID\# is described by X.0, where $X=1,2,3$,etc. For Level 2 Evaluation Criterion, the ID\# is described by X.1, X.2, X.3, etc. where ' $X$ ' is the Level 1 Evaluation Criterion ID\#.

2. Utility Function values range from 0 (least desirable) to 100 (most desirable).

3. If Level 2 Criterion are used, the sum of the Level 2 "Weighted Scores" must be multiplied by the Level I Weight to determine the Level 1 Weighted Score. 


\section{HLW Salt Disposition Systems Engineering Team \\ Level 1 Evaluation Criteria Assessment Form}

Alternative Number:

Alternative Title Caustic Side Solvent Extraction

Date: $9 / 18 / 99$

A. Evaluation Criterion

Titls.

Evaluation Criterion

Description:

Future Mission Interfaces

Maximize the support of identified potential future missions.

B. Evaluation Criterion ID \#:

(Note 1)

$\underline{3.0}$

C. Evaluation Criterion Weighted

$\mathrm{W}_{1}=$ .07

Value:

D. Utility Functions:

UF Value

(Note 2)

Utility Function (UF) Description:

UF.1 Flexible system capable of supporting identified potential future missions.

System will support can-in-can and spent fuel stabilization.

UF.3 System will not support can-in-can or spent fuel stabilization.

$\underline{0}$
E.

UF VALUE:

Explanatory Notes for UF Selected:

$$
\mathbf{V}_{1}=
$$
90

Cesium loading supports can-in-can mission and dispositions the canisters in a Federal Repository. Tank space gain supports spent fuel stabilization mission. Flexibility to expand throughout and vary feed composition. WSRC-TR-98-00370.

F. Evaluation Criterion weighted score (WS) for the Alternative:

$$
\mathrm{W}_{1} \times \mathrm{V}_{1}=\mathrm{WS} \therefore \underline{.07} \times \underline{.90}=
$$

Notes:

1. For Level 1 Evaluation Criterion, the ID\# is described by X.0, where $X=1,2,3$,etc. For Level 2 Evaluation Criterion, the ID\# is described by X.1, X.2, X.3, etc. where ' $X$ ' is the Level 1 Evaluation Criterion ID\#.

2. Utility Function values range from 0 (least desirable) to 100 (most desirable).

3. If Level 2 Criterion are used, the sum of the Level 2 "Weighted Scores" must be multiplied by the Level 1 Weight to determine the Level 1 Weighted Score. 


\section{HLW Salt Disposition Systems Engineering Team Level 1 Evaluation Criteria Assessment Form}

Alternative Number:

14

Alternative Title Caustic Side Solvent Extraction

Date: $9 / 18 / 99$

A. Evaluation Criterion Regulatory/ISMS/Environmental

Title:

Evaluation Criterion Description:

Protect personnel \& the environment from hazards \& releases of waste \& pollution by ensuring maximum application of intrinsic safety features.

B. Evaluation Criterion ID \#:

(Note 1)

C. Evaluation Criterion Weighted

$W_{1}=$ .23

Value:

4.0

D. Utility Functions:

Utility Function (UF) Value $\left(\mathrm{V}_{1}\right)=\Sigma$ Level 2 Criterion Weighted Score (WS)

(Note 2)

E. UF Value Formula: (Note 3)

$$
\mathrm{V}_{1}=\mathrm{WS}_{4.1} \pm \mathrm{WS}_{4.2}+\mathrm{WS}_{4.3}
$$

F. Evaluation Criterion weighted score for the Alternative:

$\mathrm{W}_{1} \times \mathrm{V}_{1}=$ Weighted Score $\therefore \underline{.23} \times \underline{64.00}=$ 14.72 Explanatory Notes for Weighted Score:

Notes:

1. For Level 1 Evaluation Criterion, the ID\# is described by X.0, where $X=1,2,3$,etc. For Level 2 Evaluation Criterion, the $I D \#$ is described by X.1, X.2, X.3, etc. where ' $X$ ' is the Level 1 Evaluation Criterion ID\#.

2. Utility Function values range from 0 (least desirable) to 100 (most desirable).

3. If Level 2 Criterion are used, the sum of the Level 2 "Weighted Scores" must be multiplied by the Level I Weight to determine the Level 1 Weighted Score. 


\section{HLW Salt Disposition Systems Engineering Team} Level 2 Evaluation Criteria Assessment Form

Alternative Number: 14

Alternative Title Caustic Side Solvent Extraction

Date: $9 / 18 / 99$

A. Evaluation Criterion

Public/Environment

Title:

Evaluation Criterion

Description:

Protect the public \& environment from hazards \& accidental releases of waste \& pollution by ensuring maximum application of intrinsic safety features

B. Evaluation Criterion ID

4.1

\#:

(Note 1)

C. Evaluation Criterion Weighted

$\mathbf{W}_{2}=$ .45

Value:

D. Utility Functions:

UF Value

(Note 2)

Utility Function (UF) Description:

Process is inherently safe and can be quantified/documented in Authorization Basis.

UF.2 Process has moderate hazards that are passively mitigated.

UF.3 Process has moderate hazards that are readily mitigated.

$\underline{60}$

UF.4 Process has inherent hazards that can be mitigated with Engineered Safety Features and $\underline{35}$ Administrative Controls.

UF.5 Process has inherent hazards and the risks are not quantifiable.
E.

UF VALUE:

Explanatory Notes for UF Selected:

Flammable material in relatively small volumes with a high flash point. Minimal vapor space, predominately liquid filled operations. Hydrogen source in alpha removal tank provides energy for source term dispersion. S-CLC-G-00187

F. Evaluation Criterion weighted score (WS) for the Alternative:

$$
\mathrm{W}_{2} \times \mathrm{V}_{2}=\mathrm{WS} \therefore . .45 \times 55=24.75
$$

Notes:

1. For Level 1 Evaluation Criterion, the ID\# is described by X.0, where $X=1,2,3$,etc. For Level 2 Evaluation Criterion, the ID\# is described by X.I, X.2, X.3, etc. where ' $X$ ' is the Level 1 Evaluation Criterion ID\#.

2. Utility Function values range from 0 (least desirable) to 100 (most desirable).

3. If Level 2 Criterion are used, the sum of the Level 2 "Weighted Scores" must be multiplied by the Level 1 Weight to determine the Level I Weighted Score. 


\section{HLW Salt Disposition Systems Engineering Team Level 2 Evaluation Criteria Assessment Form}

Alternative Number: 14 Alternative Title Caustic Side Solvent Extraction

Date: $9 / 18 / 99$

A. Evaluation Criterion Worker Title:

Evaluation Criterion Description: maximum application of intrinsic safety features.

B. Evaluation Criterion ID \#: 4.2 (Note 1)

C. Evaluation Criterion Weighted

$\mathbf{W}_{2}=$ .35 Value:

D. Utility Functions: UF Value Utility Function (UF) Description:

Process is inherently safe and poses no unusual worker safety hazard.

UF.3 Process has moderate hazards that are readily mitigated. $\quad \underline{60}$

UF.4 Process has inherent hazards that can be mitigated with Structures, Systems, Components and $\underline{40}$

UF.5 Process has inherent hazards and poses significant risk to worker safety that are not readily mitigated. $\underline{0}$

E. UF VALUE:

$$
V_{2}=
$$
55

Explanatory Notes for UF Selected: $\quad$ Flammable material in relatively small volumes. Eliminates benzene emissions. Minimal vapor space, predominantly liquid filled operations. Hydrogen source in alpha removal tank provides energy for source term dispersion. S-CLC-G-00187.

F. Evaluation Criterion weighted score (WS) for the Alternative:

$$
W_{2} \times V_{2}=W S \therefore . .35 \times \underline{55}=\underline{19.25}
$$

Notes:

1. For Level 1 Evaluation Criterion, the ID\# is described by X.0, where $X=1,2,3$,etc. For Level 2 Evaluation Criterion, the ID\# is described by X.1, X.2, X.3, etc. where ' $X$ ' is the Level 1 Evaluation Criterion ID\#.

2. Utility Function values range from 0 (least desirable) to 100 (most desirable).

3. If Level 2 Criterion are used, the sum of the Level 2 "Weighted Scores" must be multiplied by the Level 1 Weight to determine the Level 1 Weighted Score. 


\section{HLW Salt Disposition Systems Engineering Team} Level 2 Evaluation Criteria Assessment Form

Alternative Number: 14 Alternative Title Caustic Side Solvent Extraction

Date: $9 / 18 / 99$

A. Evaluation Criterion

Permitting

Title:

Evaluation Criterion Minimize waste generation risk \& difficulty of permitting new releases \& waste forms.

Description:

B. Evaluation Criterion ID \#:

(Note 1)

C. Evaluation Criterion Weighted

$\mathbf{W}_{2}=$ .2

Value:

D. Utility Functions:

UF Value

(Note 2)

Utility Function (UF) Description:

No new waste forms requiring permitting, eliminate one or more existing releases, no requalification $\quad \underline{100}$ of existing waste forms.

UF.2 Reduction in current releases, no additional permitting required.

$\underline{80}$

UF.3 Current flowsheet (Saltstone Facility needs repermitting due to Benzene releases).

$\underline{60}$

UF.4 Requalification of existing waste form, exceeds current release levels.

UF.5 New waste form permit required, significant increase in environmental releases requiring repermitting, high level waste retained in South Carolina

E.

UF VALUE:

$\mathbf{v}_{2}=$ 100

Explanatory Notes for UF Selected: Eliminates benzene releases. No new waste forms.

F. Evaluation Criterion weighted score (WS) for the Alternative:

$\mathrm{W}_{2} \times \mathrm{V}_{2}=\mathrm{WS} \therefore . \underline{.2} \times \underline{100}=$ 20.00

Notes:

1. For Level 1 Evaluation Criterion, the ID\# is described by X.0, where $X=1,2,3$,etc. For Level 2 Evaluation Criterion, the $I D \#$ is described by X.1, X.2, X.3, etc. where ' $X$ ' is the Level I Evaluation Criterion ID\#.

2. Utility Function values range from 0 (least desirable) to 100 (most desirable).

3. If Level 2 Criterion are used, the sum of the Level 2 "Weighted Scores" must be multiplied by the Level 1 Weight to determine the Level 1 Weighted Score. 


\section{HLW Salt Disposition Systems Engineering Team} Level 1 Evaluation Criteria Assessment Form

Alternative Number: 14 Alternative Title Caustic Side Solvent Extraction

Date: 9/18/99

A. Evaluation Criterion Engineering(Design)

Title:

Evaluation Criterion Description: Maximize the confidence that the facility meets applicable codes, standards \& required production throughput.

B. Evaluation Criterion ID \#: (Note 1)

5.0

C. Evaluation Criterion Weighted

$W_{1}=$ .2

Value:

D. Utility Functions:

Utility Function (UF) Value $\left(V_{1}\right)=\Sigma$ Level 2 Criterion Weighted Score (WS)

(Note 2)

UF Value Formula: $\quad \mathrm{V}_{1}=\mathrm{WS}_{5.1}+\mathrm{WS}_{5.2}+\mathrm{WS}_{5.3}+\mathrm{WS}_{5.4}$ (Note 3)

F. Evaluation Criterion weighted score for the Alternative: $\quad W_{1} \times V_{1}=$ Weighted Score $\therefore .2 .77 .50=$ Explanatory Notes for Weighted Score:

Notes:

1. For Level 1 Evaluation Criterion, the ID\# is described by $X .0$, where $X=1,2,3$,etc. For Level 2 Evaluation Criterion, the $I D \#$ is described by X.1, X.2, X.3, etc. where ' $X$ ' is the Level I Evaluation Criterion ID\#.

2. Utility Function values range from 0 (least desirable) to 100 (most desirable).

3. If Level 2 Criterion are used, the sum of the Level 2 "Weighted Scores" must be multiplied by the Level 1 Weight to determine the Level 1 Weighted Score. 


\section{HLW Salt Disposition Systems Engineering Team Level 2 Evaluation Criteria Assessment Form}

Alternative Number: 14 Alternative Title Caustic Side Solvent Extraction

Date: $9 / 18 / 99$

A. Evaluation Criterion Title:

Evaluation Criterion Description:

Ensure facility design considers major construction/testing methods and needs in accordance with Integrated Work Process (IWP) and Key Activities for Successful Execution (KASE).

B. Evaluation Criterion ID \#: (Note 1)

C. Evaluation Criterion Weighted

$\mathbf{W}_{2}=$ .25 Value:

D. Utility Functions:

UF Value

(Note 2)

Utility Function (UF) Description:

Facility design features and construction methods lead to simplicity of construction/testing process.

$\underline{100}$

Facility design features allows application of standard construction/testing practices (routine

$\underline{60}$ complexity).

UF.3 Facility design features and construction methods are difficult to apply due to non-standard, noncommercial methods not readily applied in radioactive environment.

E.

UF VALUE:

$\mathbf{V}_{2}=$ 85

Explanatory Notes for UF Selected: Existing modular design of contactors incorporating canyon experience lessons learned. Simple unit operations (tanks, evaporator).

F. Evaluation Criterion weighted score (WS) for the Alternative:

$$
\mathrm{W}_{2} \times \mathrm{V}_{2}=\mathrm{WS} \therefore \underline{.25} \times \underline{85}=\underline{21.25}
$$

Notes:

1. For Level 1 Evaluation Criterion, the ID\# is described by X.0, where $X=1,2,3$,etc. For Level 2 Evaluation Criterion, the ID\# is described by X.1, X.2, X.3, etc. where ' $X$ ' is the Level 1 Evaluation Criterion ID\#.

2. Utility Function values range from 0 (least desirable) to 100 (most desirable).

3. If Level 2 Criterion are used, the sum of the Level 2 "Weighted Scores" must be multiplied by the Level 1 Weight to determine the Level 1 Weighted Score. 
HLW Salt Disposition Systems Engineering Team Level 2 Evaluation Criteria Assessment Form

Alternative Number: 14 Alternative Title Caustic Side Solvent Extraction

Date: $9 / 18 / 99$

A. Evaluation Criterion Qualify

Title:

Evaluation Criterion Description:

Readily validate defined functional design requirements, regulatory requirements, final disposal forms, and Authorization Basis (AB) safety requirements.

B. Evaluation Criterion ID \#: (Note 1)

$\underline{5.2}$

C. Evaluation Criterion Weighted

$\mathbf{W}_{2}=$ .25 Value:

D. Utility Functions:

UF Value

(Note 2)

Utility Function (UF) Description:

$\underline{100}$

$\underline{70}$ Test program applie Insufficient science/engineering exists to establish firm test acceptance criteria and methods, limited direct verification.

E.

UF VALUE:

Explanatory Notes for UF Selected:

$$
\mathbf{V}_{2}=
$$
80

Majority of design attributes provide for direct verification. Process variables will require some inferred results. Modular unit testing would use some bounding acceptance data.

F. Evaluation Criterion weighted score (WS) for the Alternative:

$$
\mathrm{W}_{2} \times \mathrm{V}_{2}=\mathrm{WS} \therefore .25 \times \underline{80}=\underline{20.00}
$$

Notes:

1. For Level 1 Evaluation Criterion, the ID\# is described by X.O, where $X=1,2,3$,etc. For Level 2 Evaluation Criterion, the $I D \#$ is described by X.I, X.2, X.3, etc. where ' $X$ ' is the Level 1 Evaluation Criterion ID\#.

2. Utility Function values range from 0 (least desirable) to 100 (most desirable).

3. If Level 2 Criterion are used, the sum of the Level 2 "Weighted Scores" must be multiplied by the Level 1 Weight to determine the Level 1 Weighted Score. 


\section{HLW Salt Disposition Systems Engineering Team Level 2 Evaluation Criteria Assessment Form}

Alternative Number: 14 Alternative Title Caustic Side Solvent Extraction

Date: $9 / 18 / 99$

A. Evaluation Criterion

Title:

Evaluation Criterion Description:
Operate

Maximize ease of repeat operation/proceduralization, access for round sheets/physical verification, and upset operation management (Section R-1.4-3 of Functions \& Requirements).

B. Evaluation Criterion ID \#: (Note 1)

C. Evaluation Criterion Weighted

$\mathbf{W}_{2}=$ .25 Value:

D. Utility Functions:

UF Value

(Note 2)

Utility Function (UF) Description:

$\underline{100}$

Design allows simple, coordinated, straight forward operation with direct access to key controls interlocks, and instruments. Easy access to key equipment, maximize ALARA considerations. Minimize number of process control points.

UF.2 Design allows manageable operation with minimal complexity (Standard SRS practice).

UF.3 Design is highly coupled with minimum holdup, multiple parallel operations and fast dynamics, and $\underline{0}$ process instability.

E.

UF VALUE:

$\mathbf{V}_{2}=$ 75

Explanatory Notes for UF Selected: Comparable to current SRS canyon operation. Limited number of unit operations.

F. Evaluation Criterion weighted score (WS) for the Alternative:

$\mathrm{W}_{2} \times \mathrm{V}_{2}=\mathrm{WS} \therefore \underline{.25} \times \underline{\mathbf{7 5}}=\underline{18.75}$

Notes:

1. For Level 1 Evaluation Criterion, the ID\# is described by X.0, where $X=1,2,3$, etc. For Level 2 Evaluation Criterion, the $I D \#$ is described by X.I, X.2, X.3, etc. where ' $X$ ' is the Level I Evaluation Criterion ID\#.

2. Utility Function values range from 0 (least desirable) to 100 (most desirable).

3. If Level 2 Criterion are used, the sum of the Level 2 "Weighted Scores" must be multiplied by the Level 1 Weight to determine the Level 1 Weighted Score. 


\section{HLW Salt Disposition Systems Engineering Team} Level 2 Evaluation Criteria Assessment Form

Alternative Number: 14 Alternative Title Caustic Side Solvent Extraction

Date: $9 / 18 / 99$

A. Evaluation Criterion RAMI

Title:

Evaluation Criterion Design to maximize Reliability, Availability, Maintainability, and Inspectability. Description:

B. Evaluation Criterion ID \#: (Note 1)

$\underline{5.4}$

C. Evaluation Criterion Weighted

$\mathbf{W}_{2}=$ .25 Value:

D. Utility Functions:

UF Value

(Note 2)

Utility Function (UF) Description: $\underline{100}$

$\underline{60}$

UF.2 Design allows manageable maintenance functions with minimal complexibility (Standard SRS practice).

UF.3 Design complexity restricts maintainability and inspectability and reduces reliability, availability of $\underline{0}$ Structures, Systems, and Components. Remoteability restricts maintainability.

E.

UF VALUE:

$$
\mathbf{V}_{2}=
$$
70

Explanatory Notes for UF Selected: Latest generation of contactor has been improved to incorporate maintenance lessons learned. Alpha removal equipment size adds RAMI complexity. WSRC-RP-99-0006

F. Evaluation Criterion weighted score (WS) for the Alternative:

$$
W_{2} \times V_{2}=W S \therefore \underline{.25} \times \underline{70}=\underline{17.50}
$$

Notes:

1. For Level 1 Evaluation Criterion, the ID\# is described by X.0, where $X=1,2,3$,etc. For Level 2 Evaluation Criterion, the ID\# is described by X.I, X.2, X.3, etc. where ' $X$ ' is the Level 1 Evaluation Criterion ID\#.

2. Utility Function values range from 0 (least desirable) to 100 (most desirable).

3. If Level 2 Criterion are used, the sum of the Level 2 "Weighted Scores" must be multiplied by the Level 1 Weight to determine the Level 1 Weighted Score. 


\section{HLW Salt Disposition Systems Engineering Team \\ Level 1 Evaluation Criteria Assessment Form}

Alternative Number: 14 Alternative Title Caustic Side Solvent Extraction

Date: $9 / 18 / 99$

A. Evaluation Criterion

Title:

Evaluation Criterion

Description:

Cost/Schedule

Meet minimum combination of programmatic and technical risks and life cycle costs.

B. Evaluation Criterion ID \#: $\quad \underline{6.0}$

(Note 1)

C. Evaluation Criterion Weighted

$\mathbf{W}_{1}=$ .12

Value:

D. Utility Functions:

Utility Function (UF) Value $\left(\mathrm{V}_{1}\right)=\Sigma$ Level 2 Criterion Weighted Score (WS)

(Note 2)

UF Value Formula: (Note 3)

$$
V_{1}=\underline{W S_{6.1}}+W \underline{S}_{6.2}+W S_{6.3}
$$

F. Evaluation Criterion weighted score for the Alternative:

$W_{1} \times V_{1}=$ Weighted Score

$\underline{.12}$

$83.00=$

Explanatory Notes for Weighted Score:

Notes:

1. For Level 1 Evaluation Criterion, the ID\# is described by X.0, where $X=1,2,3$,etc. For Level 2 Evaluation Criterion, the ID\# is described by X.1, X.2, X.3, etc. where ' $X$ ' is the Level 1 Evaluation Criterion ID\#.

2. Utility Function values range from 0 (least desirable) to 100 (most desirable).

3. If Level 2 Criterion are used, the sum of the Level 2 "Weighted Scores" must be multiplied by the Level 1 Weight to determine the Level I Weighted Score. 


\section{HLW Salt Disposition Systems Engineering Team} Level 2 Evaluation Criteria Assessment Form

Alternative Number: 14 Alternative Title Caustic Side Solvent Extraction

Date: 9/18/99

A. Evaluation Criterion

Title:

Evaluation Criterion Description:

Regulatory Schedule Commitments

Maximize capability of disposing of radioactive wastes per Federal Facility Agreement (FFA) \& Site Treatment Plan (STP) schedules or earlier.

B. Evaluation Criterion ID \#: (Note 1)

$\underline{6.1}$

C. Evaluation Criterion Weighted

$\mathbf{W}_{2}=$ .5 Value:

D. Utility Functions:

UF Value

(Note 2)

Utility Function (UF) Description:

Accelerated Cleanup Plan (ACP) to empty High Level Waste (HLW) tanks by 2022 is met.

$\underline{100}$

UF.2 Base Site Treatment Plan (STP) requirement to close HLW tanks by 2028 is met.

$\underline{70}$

UF.3 Base STP or Federal Facility Agreement (FFA) requirements to close HLW tanks by committed dates $\underline{0}$ is not met.

E.

UF VALUE:

Explanatory Notes for UF Selected:

DWPF operation supports STP requirement. Flexibility to expand throughput to potentially meet ACP.

F. Evaluation Criterion weighted score (WS) for the Alternative:

$$
\mathbf{W}_{2} \times \mathrm{V}_{2}=\mathrm{WS} \therefore \underline{.5} \times \underline{\mathbf{9 0}}=
$$

45.00

Notes:

1. For Level 1 Evaluation Criterion, the ID\# is described by X.0, where $X=1,2,3$,etc. For Level 2 Evaluation Criterion, the ID\# is described by X.I, X.2, X.3, etc. where ' $X$ ' is the Level 1 Evaluation Criterion ID\#.

2. Utility Function values range from 0 (least desirable) to 100 (most desirable).

3. If Level 2 Criterion are used, the sum of the Level 2 "Weighted Scores" must be multiplied by the Level 1 Weight to determine the Level I Weighted Score. 
HLW Salt Disposition Systems Engineering Team Level 2 Evaluation Criteria Assessment Form

Alternative Number: 14 Alternative Title Caustic Side Solvent Extraction

Date: $9 / 18 / 99$

A. Evaluation Criterion Life Cycle Costs (LCC)

Title:

Evaluation Criterion Minimize LCC including TEC, OPC, and D\&D (excludes salvage and repository costs). Description:

B. Evaluation Criterion ID \#: $\quad \underline{6.2}$. (Note 1)

C. Evaluation Criterion Weighted

$\mathrm{W}_{2}=$ .3 Value:

D. Utility Functions:

UF Value

(Note 2)

Utility Function (UF) Description:

UF.1 $\quad L C C \leq 2$ billion dollars.

$\underline{50}$

UF.3 LCC is 8 billion dollars.

$\underline{25}$

UF.4 $\quad \underline{L C C} \geq 16$ billion dollars.

E.

UF VALUE:

$V_{2}=$ 60

Explanatory Notes for UF Selected: $\quad$ LCC equals 3.5 billion dollars. Based on the point estimate. WSRC-RP-99-00167.

F. Evaluation Criterion weighted score (WS) for the Alternative:

$W_{2} \times V_{2}=W S \therefore . .3 \times .60=$ 18.00

Notes:

1. For Level 1 Evaluation Criterion, the ID\# is described by X.0, where $X=1,2,3$,etc. For Level 2 Evaluation Criterion, the $I D \#$ is described by X.1, X.2, X.3, etc. where ' $X$ ' is the Level 1 Evaluation Criterion ID\#.

2. Utility Function values range from 0 (least desirable) to 100 (most desirable).

3. If Level 2 Criterion are used, the sum of the Level 2 "Weighted Scores" must be multiplied by the Level I Weight to determine the Level 1 Weighted Score. 
HLW Salt Disposition Systems Engineering Team Level 2 Evaluation Criteria Assessment Form

Alternative Number: 14 Alternative Title Caustic Side Solvent Extraction

Date: 9/18/99

A. Evaluation Criterion

Repository Costs

Title:

Evaluation Criterion

Description:

Minimize cost for waste disposal off-site (Federal Repository).

B. Evaluation Criterion ID \#: 6.3

(Note 1)

C. Evaluation Criterion Weighted

$\mathbf{W}_{2}=$

.2

Value:

D. Utility Functions:

UF Value

(Note 2)

Utility Function (UF) Description:

$\leqq 6000$ canisters for off-site disposal

$\underline{100}$

$\underline{50}$

$\underline{0}$

UF.3 $\geqq 130,000$ canisters for off-site disposal

$V_{2}=100$

E.

UF VALUE:

DWPF canister production remains at 6000 .

F. Evaluation Criterion weighted score (WS) for the Alternative:

$\mathrm{W}_{2} \times \mathrm{V}_{2}=\mathrm{WS} \therefore .2 .2 \times 100=\underline{20.00}$

Notes:

1. For Level 1 Evaluation Criterion, the ID\# is described by X.O, where $X=1,2,3$,etc. For Level 2 Evaluation Criterion, the ID\# is described by X.I, X.2, X.3, etc. where ' $X$ ' is the Level I Evaluation Criterion ID\#.

2. Utility Function values range from 0 (least desirable) to 100 (most desirable).

3. If Level 2 Criterion are used, the sum of the Level 2 "Weighted Scores" must be multiplied by the Level 1 Weight to determine the Level 1 Weighted Score. 
High Level Waste Salt Disposition

Systems Engineering Team

Final Report
WSRC-RP-99-00007

Revision: 0

Page 70 of 277

\subsubsection{CST Non-Elutable Ion Exchange}




\section{HLW Salt Disposition Systems Engineering Team} Level 1 Evaluation Criteria Assessment Form

Alternative Number: Alternative Title Crystalline Silicotitanate (CST) Ion Exchange - DWPF Vitrification

Date: $9 / 18 / 99$

A. Evaluation Criterion Technology

Title:

Evaluation Criterion Description: Maximize the confidence that underlying scientific principles \& engineering implementation will result in adequate attainment.

B. Evaluation Criterion ID \#: (Note 1)

C. Evaluation Criterion Weighted

$\mathbf{W}_{1}=$ .23

Value:

1.0

D. Utility Functions:

Utility Function (UF) Value $\left(V_{1}\right)=\Sigma$ Level 2 Criterion Weighted Score (WS)

(Note 2)

UF Value Formula:

$\mathrm{V}_{1}=\underline{\mathrm{WS}_{1.1}}+\mathrm{WS}_{\underline{1.2}}+\mathrm{WS}_{\underline{1.3}}$

(Note 3)

F. Evaluation Criterion weighted score for the Alternative:

$\mathrm{W}_{1} \times \mathrm{V}_{1}=$ Weighted Score

58.00

Explanatory Notes for Weighted Score:

Notes:

1. For Level 1 Evaluation Criterion, the ID\# is described by X.0, where $X=1,2,3$,etc. For Level 2 Evaluation Criterion, the ID\# is described by X.1, X.2, X.3, etc. where ' $X$ ' is the Level 1 Evaluation Criterion ID\#.

2. Utility Function values range from 0 (least desirable) to 100 (most desirable).

3. If Level 2 Criterion are used, the sum of the Level 2 "Weighted Scores" must be multiplied by the Level 1 Weight to determine the Level 1 Weighted Score. 


\section{HLW Salt Disposition Systems Engineering Team} Level 2 Evaluation Criteria Assessment Form

Alternative Number Alternative Title Crystalline Silicotitanate (CST) Ion Exchange - DWPF Vitrification

Date: $9 / 18 / 99$

A. Evaluation Criterion Scientific Maturity

Title:

Evaluation Criterion The level of scientific understanding needed to minimize project risk.

Description:

B. Evaluation Criterion ID \#: (Note 1)

C. Evaluation Criterion Weighted Value:

$\mathrm{W}_{2}=$ .4

D. Utility Functions:

UF Value

Utility Function (UF) Description:

(Note 2)

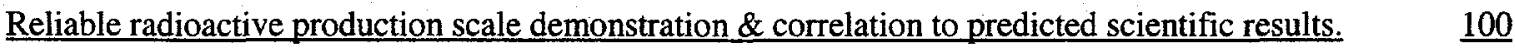

JF.2 Large scale radioactive test: 'spiked' radiochemistry demonstration.

$\underline{80}$

UF.3 Pilot (small) scale radioactive test; full radiochemistry.

$\underline{40}$

UF.4 Lab scale test: simulant/real waste.

$\underline{10}$

UF.5 Theoretical understanding only; no practical demonstration.

$\underline{0.0}$

E.

UF VALUE:

Explanatory Notes for UF Selected:

Oak Ridge large scale radioactive demonstration and numerous lab and pilot scale tests. but not with SRS high alkaline waste. DF and cesium loading has only been demonstrated with SRS waste at lab scale. R\&D results indicate performance and throughput issues which require resin re-engineering for SRS waste. DWPF glass production experience requires changes to an existing formulation and requalification. WSRC-RP-99-0006; WSRC-TR-99-00245

F. Evaluation Criterion weighted score (WS) for the Alternative:

$$
\mathrm{W}_{2} \times \mathrm{v}_{2}=\mathrm{WS} \therefore \underline{.4} \times \underline{\mathbf{4 0}}=
$$

Notes:

1. For Level 1 Evaluation Criterion, the ID\# is described by X.0, where $X=1,2,3$,etc. For Level 2 Evaluation Criterion, the $I D \#$ is described by X.I, X.2, X.3, etc. where ' $X$ ' is the Level 1 Evaluation Criterion ID\#.

2. Utility Function values range from 0 (least desirable) to 100 (most desirable).

3. If Level 2 Criterion are used, the sum of the Level 2 "Weighted Scores" must be multiplied by the Level 1 Weight to determine the Level 1 Weighted Score. 


\section{HLW Salt Disposition Systems Engineering Team Level 2 Evaluation Criteria Assessment Form}

Alternative Number: 6 Alternative Title__ Crystalline Silicotitanate (CST) Ion Exchange - DWPF Vitrification

Date: $9 / 18 / 99$

A. Evaluation Criterion

Engineering Maturity

Title:

Evaluation Criterion The level of applied engineering concepts needed to minimize project risk

Description:

B. Evaluation Criterion ID \#:

(Nóte 1)

C. Evaluation Criterion Weighted

$\mathbf{W}_{2}=$ .4

Value:

D. Utility Functions:

UF Value

(Note 2)

Utility Function (UF) Description:

UF.1 Reliable radioactive production scale with significant operating experience

$\underline{100}$

$\underline{60}$

$\underline{40}$

UF.3 Limited radioactive production scale.

$\underline{20}$

UF.4 Limited non-radioactive production scale

$\underline{0}$
E.

UF VALUE:

Explanatory Notes for UF Selected:

Oak Ridge experience in radioactive production. DWPF glass production experience. West Valley radioactive production experience. Loaded resin requires size reduction which leads to resuspension uncertainties in the downstream process. Limited experience in high radiation field work with carousel configuration. Alpha removal process provides some engineering challenges in the areas of filtration, mixing, and pumping. WSRC-RP99-0006: WSRC-RT-99-00342, June 1,1998 West Valley Trip Report

F. Evaluation Criterion weighted score (WS) for the Alternative: $\quad W_{2} \times V_{2}=W S \therefore .4 \times 70=\frac{\mathbf{7 0 . 0 0}}{2}$

Notes:

1. For Level I Evaluation Criterion, the ID\# is described by X.0, where $X=1,2,3$,etc. For Level 2 Evaluation Criterion, the ID\# is described by X.1, X.2, X.3, etc. where ' $X$ ' is the Level 1 Evaluation Criterion ID\#.

2. Utility Function values range from 0 (least desirable) to 100 (most desirable).

3. If Level 2 Criterion are used, the sum of the Level 2 "Weighted Scores" must be multiplied by the Level 1 Weight to determine the Level 1 Weighted Score. 


\section{HLW Salt Disposition Systems Engineering Team Level 2 Evaluation Criteria Assessment Form}

Alternative Number: 6 Alternative Title__ Crystalline Silicotitanate (CST) Ion Exchange - DWPF Vitrification

Date: $9 / 18 / 99$

A. Evaluation Criterion

Process Simplicity

Title:

Evaluation Criterion

Ease of science implementation understanding by operators

Description:

B. Evaluation Criterion ID \#:

(Note 1)

C. Evaluation Criterion Weighted

$\mathbf{W}_{2}=$ .2

Value:

D. Utility Functions:

UF Value

(Note 2)

Utility Function (UF) Description:

UF.1 Low complexity, straight forward operations.

$\underline{100}$

Moderate complexity - operator aids and routine engineering support.

$\underline{70}$

UF.3 Complex - significant training for operators and continuous. specialized engineering support required.

$\underline{0.0}$

E.

UF VALUE:

Explanatory Notes for UF Selected:

Several unit operations which are straight forward with added coupling to DWPF sludge only operations.

F. Evaluation Criterion weighted score (WS) for the Alternative:

$\mathrm{W}_{2} \times \mathrm{V}_{2}=\mathrm{WS} \therefore \underline{.2} \times \underline{70}=$

14.00

Notes:

1. For Level 1 Evaluation Criterion, the ID\# is described by X.O, where $X=1,2,3$, etc. For Level 2 Evaluation Criterion, the $I D \#$ is described by X.I, X.2, X.3, etc. where ' $X$ ' is the Level 1 Evaluation Criterion ID\#.

2. Utility Function values range from 0 (least desirable) to 100 (most desirable).

3. If Level 2 Criterion are used, the sum of the Level 2 "Weighted Scores" must be multiplied by the Level I Weight to determine the Level 1 Weighted Score. 


\section{HLW Salt Disposition Systems Engineering Team} Level 1 Evaluation Criteria Assessment Form

Alternative Number: 6 Alternative Title Crystalline Silicotitanate (CST) Ion Exchange - DWPF Vitrification

Date: $9 / 18 / 99$

A. Evaluation Criterion Current Mission Interfaces

Title:

Evaluation Criterion Description: Impact on current SRS missions/programs.

B. Evaluation Criterion ID \#: (Note 1)

2.0

C. Evaluation Criterion Weighted

$W_{1}=$ .15 Value:

D. Utility Functions:

Utility Function (UF) Value $\left(\mathrm{V}_{1}\right)=\Sigma$ Level 2 Criterion Weighted Score (WS)

(Note 2)

UF Value Formula: (Note 3)

$$
V_{1}=W S_{2.1}+W S_{2.2}+W S_{2.3}+W S_{2.4}+W S_{2.5}
$$

F. Evaluation Criterion weighted score for the Alternative:

$$
\mathrm{W}_{1} \times \mathrm{V}_{1}=\text { Weighted Score } \therefore . .15 \times \underline{60.50}=
$$

Explanatory Notes for Weighted Score:

Notes:

1. For Level 1 Evaluation Criterion, the ID\# is described by X.0, where $X=1,2,3$,etc. For Level 2 Evaluation Criterion, the ID\# is described by X.1, X.2, X.3, etc. where ' $X$ ' is the Level I Evaluation Criterion ID\#.

2. Utility Function values range from 0 (least desirable) to 100 (most desirable).

3. If Level 2 Criterion are used, the sum of the Level 2 "Weighted Scores" must be multiplied by the Level 1 Weight to determine the Level 1 Weighted Score. 


\section{HLW Salt Disposition Systems Engineering Team Level 2 Evaluation Criteria Assessment Form}

Alternative Number: Alternative Title Crystalline Silicotitanate (CST) Ion Exchange - DWPF Vitrification

Date: $9 / 18 / 99$

A. Evaluation Criterion Titla. Evaluation Criterion Description:

\section{DWPF}

Impact on DWPF (Table 1 Functions \& Requirements).

C. Evaluation Criterion ID \#: (Note 1)

C. Evaluation Criterion Weighted

$\mathrm{W}_{2}=$ .25 Value:

E. Utility Functions:

UF Value

(Note 2)

Utility Function (UF) Description:

UF.1 Sludge only to completion

Sludge plus MST to completion.

$\underline{85}$

UF.3 Baseline - current ITP flowsheet.

$\underline{70}$

UF.4 Moderate impact - some additional canisters $(<50 \%)$. Facility modifications required. $\underline{20}$

UF.5 Significant impact - additional canisters $(>50 \%)$ glass reformulation/repermitting required. Major facility modifications required.

E.

UF VALUE:

$\mathrm{V}_{2}=$ 40

Explanatory Notes for UF Selected:

CST and MST added to the DWPF sludge only flowsheet. Late Wash Facility and Salt Process Cell operation are not required. Loaded resin requires size reduction which leads to resuspension and sampling efficacy uncertainties in DWPF. Glass requalification is required to address glass viscosity, model refinements and possible reformulation. WSRCTR-99-00245; WSRC-TR-99-00309; WSRC-TR-99-00302; WSRC-RP-99-0006

F. Evaluation Criterion weighted score (WS) for the Alternative:

$$
\mathrm{W}_{2} \times \mathrm{V}_{2}=\mathrm{WS} \therefore \underline{.25} \times \underline{40}=10.00
$$

Notes:

1. For Level 1 Evaluation Criterion, the ID\# is described by X.0, where $X=1,2,3$,etc. For Level 2 Evaluation Criterion, the ID\# is described by X.1, X.2, X.3, etc. where ' $X$ ' is the Level 1 Evaluation Criterion ID\#.

2. Utility Function values range from 0 (least desirable) to 100 (most desirable).

3. If Level 2 Criterion are used, the sum of the Level 2 "Weighted Scores" must be multiplied by the Level 1 Weight to determine the Level 1 Weighted Score. 


\section{HLW Salt Disposition Systems Engineering Team Level 2 Evaluation Criteria Assessment Form}

Alternative Number:

Alternative Title Crystalline Silicotitanate (CST) Ion Exchange - DWPF Vitrification

Date: $9 / 18 / 99$

A. Evaluation Criterion Saltstone

Title:

Evaluation Criterion Impact on Saltstone (Table 1 Functions \& Requirements)

Description:

B. Evaluation Criterion ID \#:

(Note 1)

C. Evaluation Criterion Weighted

$\mathbf{W}_{2}=$ .15

Value:

D. Utility Functions:

UF Value

(Note 2)

Utility Function (UF) Description:

No need for Saltstone Facility.

$\underline{100}$

$\underline{80}$

$\underline{70}$

$\underline{40}$

UF.4 Moderate increase in saltstone ( $<50 \%$ ). Minor facility modifications.

$\underline{0}$
E.

UF VALUE:

Explanatory Notes for UF Selected:

$\mathbf{V}_{2}=$ 80

Reduction in saltstone production by 30 million gallons of saltstone grout. No benzene release

F. Evaluation Criterion weighted score (WS) for the Alternative:

$\mathrm{W}_{2} \times \mathrm{V}_{2}=\mathrm{WS} \therefore \underline{.15} \times \underline{80}=\underline{12.00}$

Notes:

1. For Level 1 Evaluation Criterion, the ID\# is described by X.0, where $X=1,2,3$,etc. For Level 2 Evaluation Criterion, the $I D \#$ is described by X.1, X.2, X.3, etc. where ' $X$ ' is the Level 1 Evaluation Criterion ID\#.

2. Utility Function values range from 0 (least desirable) to 100 (most desirable).

3. If Level 2 Criterion are used, the sum of the Level 2 "Weighted Scores" must be multiplied by the Level 1 Weight to determine the Level 1 Weighted Score. 


\section{HLW Salt Disposition Systems Engineering Team \\ Level 2 Evaluation Criteria Assessment Form}

Alternative Number: Alternative Title Crystalline Silicotitanate (CST) Ion Exchange - DWPF Vitrification

Date: $9 / 18 / 99$

A. Evaluation Criterion Solid Waste

Title:

Evaluation Criterion Description:

B. Evaluation Criterion ID \#:

(Note 1)

C. Evaluation Criterion Weighted

$\mathbf{W}_{2}=$ .1

Value:

D. Utility Functions:

UF Value

(Note 2)

Utility Function (UF) Description:

Reduced solid waste volume and no Benzene.

$\underline{100}$

Reduced solid waste volume and Benzene.

$\underline{80}$

UF.2

Current flowsheet (Benzene to CIF).

$\underline{50}$

$\underline{30}$

UF.4 Moderate increase in solid waste volume.

$\underline{0}$

UF.5 Repermit new waste forms, significant increase in solid waste volume.

E. UF VALUE:

$\mathrm{V}_{2}=$ 80

Explanatory Notes for UF Selected: Reduction in liquid benzene generation by 35,000 gallons per year (no benzene generated).

F. Evaluation Criterion weighted score (WS) for the Alternative:

$\mathrm{W}_{2} \times \mathrm{V}_{2}=\mathrm{WS} \therefore .1 \times \underline{80}=$

8.00

Notes:

1. For Level 1 Evaluation Criterion, the ID\# is described by X.O, where $X=1,2,3$,etc. For Level 2 Evaluation Criterion, the $I D \#$ is described by X.1, X.2, X.3, etc. where ' $X$ ' is the Level I Evaluation Criterion ID\#.

2. Utility Function values range from 0 (least desirable) to 100 (most desirable).

3. If Level 2 Criterion are used, the sum of the Level 2 "Weighted Scores" must be multiplied by the Level 1 Weight to determine the Level 1 Weighted Score. 


\section{HLW Salt Disposition Systems Engineering Team Level 2 Evaluation Criteria Assessment Form}

Alternative Number: Alternative Title Crystalline Silicotitanate (CST) Ion Exchange - DWPF Vitrification

Date: $9 / 18 / 99$

A. Evaluation Criterion Tank Farm

Title:

Evaluation Criterion Impact on Tank Farm (Table 1 Functions \& Requirements).

Description:

B. Evaluation Criterion ID \#: (Note 1)

2.4

C. Evaluation Criterion Weighted

$\mathbf{W}_{2}=$ .2 Value:

D. Utility Functions:

UF Value

(Note 2)

Utility Function (UF) Description: $\underline{100}$

$\underline{50}$ impact. Current flowsheet.

UF.3 Increased safety hazards (e.g. Organics) increase operational capacity, increased corrosion impacts. $\quad \underline{0}$
E.

UF VALUE:

Explanatory Notes for UF Selected:
$V_{2}=$ 70

Reduced organics transferred to the Tank Farm. Reduced recycle stream relative to the base case ITP.

F. Evaluation Criterion weighted score (WS) for the Alternative:

$$
\mathrm{W}_{2} \times \mathrm{V}_{2}=\mathrm{WS} \therefore \underline{.2} \times \underline{70}=
$$

Notes:

1. For Level I Evaluation Criterion, the ID\# is described by X.O, where $X=1,2,3$,etc. For Level 2 Evaluation Criterion, the ID\# is described by X.1, X.2, X.3, etc. where ' $X$ ' is the Level 1 Evaluation Criterion ID\#.

2. Utility Function values range from 0 (least desirable) to 100 (most desirable).

3. If Level 2 Criterion are used, the sum of the Level 2 "Weighted Scores" must be multiplied by the Level 1 Weight to determine the Level I Weighted Score. 


\section{HLW Salt Disposition Systems Engineering Team} Level 2 Evaluation Criteria Assessment Form

Alternative Number Alternative Title Crystalline Silicotitanate (CST) Ion Exchange - DWPF Vitrification

Date: $9 / 18 / 99$

A. Evaluation Criterion Tank Farm Space Management

Title:

Utilization of available Tank Farm storage \& resources as a function of time (HLW Salt

Evaluation Criterion Description: Disposition Interface Functional Performance Requirement).

B. Evaluation Criterion ID \#: (Note 1)

$\underline{2.5}$

C. Evaluation Criterion Weighted

$\mathbf{W}_{2}=$ .3 Value:

D. Utility Functions:

Utility Function (UF) Description:

UF Value

(Note 2)

$\underline{100}$

$\underline{40}$

JF.2 Current flowsheet (reduces available tank space)

$\underline{0}$

UF.3 Accelerated reduction in available tank space (water logged tank farm).
E.

UF VALUE:

Explanatory Notes for UF Selected:

F. Evaluation Criterion weighted score (WS) for the Alternative:

$$
\mathbf{V}_{\mathbf{2}}=
$$
55

TK49 readily available for waste storage. TK48 available after waste handling strategy is completed. Reduced recycle volume. WSRC-RP-99-0005; WSRC-RP-99-0006

Notes:

1. For Level 1 Evaluation Criterion, the ID\# is described by X.0, where $X=1,2,3$,etc. For Level 2 Evaluation Criterion, the ID\# is described by X.I, X.2, X.3, etc. where ' $X$ ' is the Level 1 Evaluation Criterion ID\#.

2. Utility Function values range from 0 (least desirable) to 100 (most desirable).

3. If Level 2 Criterion are used, the sum of the Level 2 "Weighted Scores" must be multiplied by the Level 1 Weight to determine the Level 1 Weighted Score. 


\section{HLW Salt Disposition Systems Engineering Team Level 1 Evaluation Criteria Assessment Form}

Alternative Number:

Alternative Title

Crystalline Silicotitanate (CST) Ion Exchange - DWPF Vitrification

Date: 9/18/99

A. Evaluation Criterion Future Mission Interfaces

Title:

Evaluation Criterion Maximize the support of identified potential future missions.

Description:

B. Evaluation Criterion ID \#:

(Note 1)

C. Evaluation Criterion Weighted

$\mathbf{W}_{1}=$ .07

Value:

D. Utility Functions:

UF Value

Utility Function (UF) Description:

(Note 2)

Flexible system capable of supporting identified potential future missions.

$\underline{100}$

System will support can-in-can and spent fuel stabilization.

$\underline{70}$

UF.3 System will not support can-in-can or spent fuel stabilization.

$\underline{0}$

E.

UF VALUE:

Explanatory Notes for UF Selected:
$V_{1}=$ 70

Cesium loading supports can-in-can mission and dispositions the canisters in a Federal Repository. Tank space gain supports spent fuel stabilization mission.

F. Evaluation Criterion weighted score (WS) for the Alternative:

$\mathrm{W}_{1} \times \mathrm{V}_{1}=\mathrm{WS} \therefore \underline{.07} \times \underline{\mathbf{7 0}}=$ 4.90

Notes:

1. For Level 1 Evaluation Criterion, the ID\# is described by X.O, where $X=1,2,3$,etc. For Level 2 Evaluation Criterion, the $I D \#$ is described by X.1, X.2, X.3, etc. where ' $X$ ' is the Level 1 Evaluation Criterion ID\#.

2. Utility Function values range from 0 (least desirable) to 100 (most desirable).

3. If Level 2 Criterion are used, the sum of the Level 2 "Weighted Scores" must be multiplied by the Level I Weight to determine the Level 1 Weighted Score. 


\section{HLW Salt Disposition Systems Engineering Team} Level 1 Evaluation Criteria Assessment Form

Alternative Number: Alternative Title Crystalline Silicotitanate (CST) Ion Exchange - DWPF Vitrification

Date: $9 / 18 / 99$

A. Evaluation Criterion Regulatory/ISMS/Environmental

Title:

Evaluation Criterion Description:

Protect personnel \& the environment from hazards \& releases of waste \& pollution by ensuring maximum application of intrinsic safety features.

B. Evaluation Criterion ID \#: (Note 1)

C. Evaluation Criterion Weighted

$\mathbf{W}_{1}=$ .23 Value:

D. Utility Functions:

Utility Function (UF) Value $\left(\mathrm{V}_{1}\right)=\Sigma$ Level 2 Criterion Weighted Score (WS)

(Note 2)

UF Value Formula: $\quad V_{1}=\underline{W S}_{4.1}+W_{4.2}+W_{4.3}$ (Note 3)

F. Evaluation Criterion weighted score for the Alternative: $\quad W_{1} \times V_{1}=$ Weighted Score $\therefore . .23 \times \frac{39.75}{=}=$ Explanatory Notes for Weighted Score:

Notes:

1. For Level 1 Evaluation Criterion, the ID\# is described by X.0, where $X=1,2,3$,etc. For Level 2 Evaluation Criterion, the ID\# is described by X.I, X.2, X.3, etc. where ' $X$ ' is the Level I Evaluation Criterion ID\#.

2. Utility Function values range from 0 (least desirable) to 100 (most desirable).

3. If Level 2 Criterion are used, the sum of the Level 2 "Weighted Scores" must be multiplied by the Level I Weight to determine the Level 1 Weighted Score. 


\section{HLW Salt Disposition Systems Engineering Team Level 2 Evaluation Criteria Assessment Form}

Alternative Number:

Alternative Title

Crystalline Silicotitanate (CST) Ion Exchange - DWPF Vitrification

Date: $9 / 18 / 99$

A. Evaluation Criterion

Public/Environment

Title:

Evaluation Criterion

Description:

Protect the public \& environment from hazards \& accidental releases of waste \& pollution by ensuring maximum application of intrinsic safety features.

B. Evaluation Criterion ID \#:

(Note 1)

C. Evaluation Criterion Weighted

$\mathbf{W}_{2}=$ .45

Value:

D. Utility Functions:

UF Value

(Note 2)

Utility Function (UF) Description:

Process is inherently safe and can be quantified/documented in Authorization Basis.

$\underline{100}$

Process has moderate hazards that are passively mitigated.

$\underline{85}$

UF.3 Process has moderate hazards that are readily mitigated.

$\underline{60}$

UF.4 Process has inherent hazards that can be mitigated with Engineered Safety Features and

$\underline{35}$

Administrative Controls.

UF.5 Process has inherent hazards and the risks are not quantifiable.

$\underline{0}$

E.

UF VALUE:

$\mathbf{V}_{2}=$ 35

Explanatory Notes for UF Selected:

Higher source term cesium loading on resin leads to temperature management concerns and large quantities of hydrogen and oxygen concentrations in detonable levels. WSRCTR-99-00285; No benzene in the process. Hydrogen source in alpha removal tank provides energy for source term dispersion. WSRC-RP-99-0006

F. Evaluation Criterion weighted score (WS) for the Alternative:

$$
W_{2} \times V_{2}=W S \therefore .45 \times \underline{.45}=15.75
$$

Notes:

1. For Level 1 Evaluation Criterion, the ID\# is described by X.O, where $X=1,2,3$,etc. For Level 2 Evaluation Criterion, the $I D \#$ is described by X.I, X.2, X.3, etc. where ' $X$ ' is the Level 1 Evaluation Criterion ID\#.

2. Utility Function values range from 0 (least desirable) to 100 (most desirable).

3. If Level 2 Criterion are used, the sum of the Level 2 "Weighted Scores" must be multiplied by the Level 1 Weight to determine the Level 1 Weighted Score. 


\section{HLW Salt Disposition Systems Engineering Team} Level 2 Evaluation Criteria Assessment Form

Alternative Number

Alternative Title

Crystalline Silicotitanate (CST) Ion Exchange - DWPF Vitrification

Date: $9 / 18 / 99$

A. Evaluation Criterion

Worker

Title:

Evaluation Criterion

Description:

Protect on-site personnel from hazards \& accidental releases of waste \& pollution by ensuring maximum application of intrinsic safety features.

B. Evaluation Criterion ID \#:

(Note 1)

4.2

C. Evaluation Criterion Weighted

Value:

$\mathbf{W}_{2}=$ .35

D. Utility Functions:

UF Value

Utility Function (UF) Description:

(Note 2)

UF.1 Process is inherently safe and poses no unusual worker safety hazard. $\quad \underline{100}$

JF.2 Process has moderate hazards that are passively mitigated.

UF.3 Process has moderate hazards that are readily mitigated.

UF.4 Process has inherent hazards that can be mitigated with Structures, Systems, Components and

$\underline{40}$ Administrative Controls.

UF.5 Process has inherent hazards and poses significant risk to worker safety that are not readily mitigated. $\underline{0}$

E

UF VALUE:

Explanatory Notes for UF Selected:
$\mathbf{V}_{2}=$ 40

No benzene hazard. Higher source term cesium loading on resin leads to temperature management concerns and large quantities of hydrogen and oxygen concentrations in detonable levels. Hydrogen source in alpha removal tank provides energy for source term dispersion. WSRC-TR-99-00285; WSRC-RP-99-0006; S-CLC-G-00187

F. Evaluation Criterion weighted score (WS) for the Alternative:

$$
\mathbf{W}_{2} \times \mathrm{V}_{2}=\mathbf{W S} \therefore \underline{.35} \times \underline{40}=
$$

Notes:

1. For Level 1 Evaluation Criterion, the ID\# is described by X.O, where $X=1,2,3$,etc. For Level 2 Evaluation Criterion, the ID\# is described by X.1, X.2, X.3, etc. where ' $X$ ' is the Level 1 Evaluation Criterion ID\#.

2. Utility Function values range from 0 (least desirable) to 100 (most desirable).

3. If Level 2 Criterion are used, the sum of the Level 2 "Weighted Scores" must be multiplied by the Level 1 Weight to determine the Level 1 Weighted Score. 


\section{HLW Salt Disposition Systems Engineering Team Level 2 Evaluation Criteria Assessment Form}

Alternative Number:_ 6 Alternative Title Crystalline Silicotitanate (CST) Ion Exchange - DWPF Vitrification

Date: $9 / 18 / 99$

A. Evaluation Criterion

Title:

Evaluation Criterion

Permitting

Description:

Minimize waste generation risk \& difficulty of permitting new releases \& waste forms.

B. Evaluation Criterion ID \#: (Note 1)

4.3

C. Evaluation Criterion Weighted

$W_{2}=$ .2

Value:

D. Utility Functions:

UF Value

(Note 2)

Utility Function (UF) Description:

UF.1 No new waste forms requiring permitting, eliminate one or more existing releases, no requalification $\quad 100$ of existing waste forms.

Reduction in current releases, no additional permitting required.

UF.3 Current flowsheet (Saltstone Facility needs repermitting due to Benzene releases).

UF.4 Requalification of existing waste form, exceeds current release levels.

UF.5 New waste form permit required, significant increase in environmental releases requiring $\underline{0}$

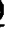

E. repermitting, high level waste retained in South Carolina

UF VALUE:

Explanatory Notes for UF Selected:
Eliminates benzene releases. Requalification of DW
Reduction in NO
emissions. WSRC-TR-99-00245

F. Evaluation Criterion weighted score (WS) for the Alternative: $\quad \mathbf{W}_{2} \times \mathbf{V}_{2}=\mathbf{W S} \therefore . \mathbf{2} \times \underline{50}=\underline{10.00}$

Notes:

1. For Level 1 Evaluation Criterion, the ID\# is described by X.O, where $X=1,2,3$,etc. For Level 2 Evaluation Criterion, the $I D \#$ is described by X.1, X.2, X.3, etc. where ' $X$ ' is the Level I Evaluation Criterion ID\#.

2. Utility Function values range from 0 (least desirable) to 100 (most desirable).

3. If Level 2 Criterion are used, the sum of the Level 2 "Weighted Scores" must be multiplied by the Level 1 Weight to determine the Level I Weighted Score. 


\section{HLW Salt Disposition Systems Engineering Team Level 1 Evaluation Criteria Assessment Form}

Alternative Number: Alternative Title Crystalline Silicotitanate (CST) Ion Exchange - DWPF Vitrification

Date: $9 / 18 / 99$

A. Evaluation Criterion Engineering (Design)

Title:

Evaluation Criterion Description:

Maximize the confidence that the facility meets applicable codes, standards \& required production throughput.

B. Evaluation Criterion ID \#: (Note 1)

$\underline{5.0}$

C. Evaluation Criterion Weighted

$$
\mathrm{W}_{1}=
$$
.2

Value:

D. Utility Functions:

Utility Function (UF) Value $\left(\mathrm{V}_{1}\right)=\Sigma$ Level 2 Criterion Weighted Score (WS)

(Note 2)

UF Value Formula: $\quad \mathbf{V}_{1}=\mathbf{W S}_{5.1}+\mathbf{W S}_{5.2}+\mathbf{W S}_{5.3}+\mathbf{W S}_{5.4}$

(Note 3)

F. Evaluation Criterion weighted score for the Alternative: $\quad W_{1} \times V_{1}=$ Weighted Score $\therefore . .2 \times 52.50=$ Explanatory Notes for Weighted Score:

Notes:

1. For Level I Evaluation Criterion, the ID\# is described by X.0, where $X=1,2,3$,etc. For Level 2 Evaluation Criterion, the $I D \#$ is described by X.1, X.2, X.3, etc. where ' $X$ ' is the Level 1 Evaluation Criterion ID\#.

2. Utility Function values range from 0 (least desirable) to 100 (most desirable).

3. If Level 2 Criterion are used, the sum of the Level 2 "Weighted Scores" must be multiplied by the Level 1 Weight to determine the Level I Weighted Score. 


\section{HLW Salt Disposition Systems Engineering Team}

Level 2 Evaluation Criteria Assessment Form

Alternative Number:

6 Alternative Title Crystalline Silicotitanate (CST) Ion Exchange - DWPF Vitrification

Date: $9 / 18 / 99$

A. Evaluation Criterion

Construct

Title:

Evaluation Criterion

Description:

Ensure facility design considers major construction/testing methods and needs in accordance with Integrated Work Process (IWP) and Key Activities for Successful Execution (KASE).

B. Evaluation Criterion ID \#:

(Note 1)

$\underline{5.1}$

C. Evaluation Criterion Weighted

$\mathbf{W}_{2}=$ .25

Value:

D. Utility Functions:

UF Value

Utility Function (UF) Description:

(Note 2)

Facility design features and construction methods lead to simplicity of construction/testing process.

$\underline{100}$

F.2 Facility design features allows application of standard construction/testing practices (routine

$\underline{60}$ complexity).

UF.3 Facility design features and construction methods are difficult to apply due to non-standard, non- $\quad \underline{0}$ commercial methods not readily applied in radioactive environment.

E.

UF VALUE:

Explanatory Notes for UF Selected:

$$
\mathbf{V}_{2}=
$$
40
Material handling complexity is increased with size reduced loaded resin. Modification within an operating facility is required for DWPF sampling systems and potentially melter feed system. WSRC-TR99-00309. WSRC-TR-99-00302.

F. Evaluation Criterion weighted score (WS) for the Alternative:

$$
\mathrm{W}_{2} \times \mathrm{V}_{2}=\mathrm{WS} \therefore \underline{.25} \times \underline{40}=\underline{10.00}
$$

Notes:

1. For Level 1 Evaluation Criterion, the ID\# is described by X.0, where $X=1,2,3$,etc. For Level 2 Evaluation Criterion, the $I D \#$ is described by X.I, X.2, X.3, etc. where ' $X$ ' is the Level I Evaluation Criterion ID\#.

2. Utility Function values range from 0 (least desirable) to 100 (most desirable).

3. If Level 2 Criterion are used, the sum of the Level 2 "Weighted Scores" must be multiplied by the Level 1 Weight to determine the Level 1 Weighted Score. 


\section{HLW Salt Disposition Systems Engineering Team Level 2 Evaluation Criteria Assessment Form}

Alternative Number

Alternative Title

Crystalline Silicotitanate (CST) Ion Exchange - DWPF Vitrification

Date: $9 / 18 / 99$

A. Evaluation Criterion

Titla.

Evaluation Criterion Description:

Qualify

Readily validate defined functional design requirements, regulatory requirements, final disposal forms, and Authorization Basis (AB) safety requirements.

B. Evaluation Criterion ID \#: (Note 1)

C. Evaluation Criterion Weighted

$\mathbf{W}_{2}=$ .25 Value:

D. Utility Functions:

UF Value

(Note 2)

Utility Function (UF) Description: $\underline{100}$

$\underline{70}$

Test program applies

Insufficient science/engineering exists to establish firm test acceptance criteria and methods, limited direct verification.

E.

UF VALUE:

$V_{2}=$ 70

Explanatory Notes for UF Selected: Interfacing with an operating facility will restrict some direct verification of design attributes. Hydrogen evolution rates in DWPF would be inferred through laboratory results.

F. Evaluation Criterion weighted score (WS) for the Alternative:

$W_{2} \times V_{2}=W S \therefore .25 \times \underline{70}=17.50$

Notes:

1. For Level I Evaluation Criterion, the ID\# is described by X.0, where $X=1,2,3$,etc. For Level 2 Evaluation Criterion, the ID\# is described by X.1, X.2, X.3, etc. where ' $X$ ' is the Level I Evaluation Criterion ID\#.

2. Utility Function values range from 0 (least desirable) to 100 (most desirable).

3. If Level 2 Criterion are used, the sum of the Level 2 "Weighted Scores" must be multiplied by the Level 1 Weight to determine the Level 1 Weighted Score. 


\section{HLW Salt Disposition Systems Engineering Team Level 2 Evaluation Criteria Assessment Form}

Alternative Number: Alternative Title Crystalline Silicotitanate (CST) Ion Exchange - DWPF Vitrification

Date: $9 / 18 / 99$

A. Evaluation Criterion

Title:

Evaluation Criterion

Description:

Operate

Maximize ease of repeat operation/proceduralization, access for round sheets/physical verification, and upset operation management (Section R-1.4-3 of Functions \& Requirements).

B. Evaluation Criterion ID \#:

(Note 1)

C. Evaluation Criterion Weighted

$\mathbf{W}_{2}=$ .25

Value:

D. Utility Functions:

Utility Function (UF) Description:

UF Value

(Note 2)

$\underline{100}$ interlocks, and instruments. Easy access to key equipment, maximize ALARA considerations. Minimize number of process control points.

UF.2 Design allows manageable operation with minimal complexity (Standard SRS practice).

$\underline{60}$

UF.3 Design is highly coupled with minimum holdup, multiple parallel operations and fast dynamics, and process instability.

E.

UF VALUE:

Explanatory Notes for UF Selected:

Material handling is routine complexity. Additional operational restrictions for DWPF (glass formulation, sampling, resin grinder).

F. Evaluation Criterion weighted score (WS) for the Alternative:

$$
\mathbf{W}_{2} \times \mathrm{V}_{2}=\mathrm{WS} \therefore . .25 \times \underline{50}=
$$

Notes:

1. For Level 1 Evaluation Criterion, the ID\# is described by X.O, where $X=1,2,3$,etc. For Level 2 Evaluation Criterion, the $I D \#$ is described by X.1, X.2, X.3, etc. where ' $X$ ' is the Level 1 Evaluation Criterion ID\#.

2. Utility Function values range from 0 (least desirable) to 100 (most desirable).

3. If Level 2 Criterion are used, the sum of the Level 2 "Weighted Scores" must be multiplied by the Level 1 Weight to determine the Level 1 Weighted Score. 


\section{HLW Salt Disposition Systems Engineering Team Level 2 Evaluation Criteria Assessment Form}

Alternative Number:_6 Alternative Title__Crystalline Silicotitanate (CST) Ion Exchange - DWPF Vitrification

Date: $9 / 18 / 99$

A. Evaluation Criterion RAMI

Title:

Evaluation Criterion

Design to maximize Reliability, Availability, Maintainability, and Inspectability.

Description:

B. Evaluation Criterion ID \#: $\quad 5.4$

(Note 1)

C. Evaluation Criterion Weighted

$\mathbf{W}_{2}=$ .25

Value:

D. Utility Functions:

UF Value

(Note 2)

Utility Function (UF) Description:

UF.1 Design allows simple, coordinated, straight forward maintenance practices which take into account

ALARA requirements. Design maximizes reliability, and availability of Structures, Systems, and

Components.

UF.2 Design allows manageable maintenance functions with minimal complexibility (Standard SRS practice).

UF.3 Design complexity restricts maintainability and inspectability and reduces reliability, availability of Structures, Systems, and Components. Remoteability restricts maintainability.

E.

UF VALUE:

Explanatory Notes for UF Selected:
$\mathrm{V}_{2}=$ 50

Material handling concerns. Similar complexity to standard SRS practices. Alpha removal equipment size, temperature management and other unique equipment adds RAMI complexity.

F. Evaluation Criterion weighted score (WS) for the Alternative:

$$
\mathrm{W}_{2} \times \mathrm{V}_{2}=\mathrm{WS} \therefore .25 \times \underline{50}=\underline{12.50}
$$

Notes:

1. For Level 1 Evaluation Criterion, the ID\# is described by X.0, where $X=1,2,3$,etc. For Level 2 Evaluation Criterion, the ID\# is described by X.1, X.2, X.3, etc. where ' $X$ ' is the Level 1 Evaluation Criterion ID\#.

2. Utility Function values range from 0 (least desirable) to 100 (most desirable).

3. If Level 2 Criterion are used, the sum of the Level 2 "Weighted Scores" must be multiplied by the Level 1 Weight to determine the Level 1 Weighted Score. 


\section{HLW Salt Disposition Systems Engineering Team \\ Level 1 Evaluation Criteria Assessment Form}

Alternative Number: $\quad 6$ Alternative Title Crystalline Silicotitanate (CST) Ion Exchange - DWPF Vitrification

Date: 9/18/99

A. Evaluation Criterion Cost/Schedule

Title:

Evaluation Criterion

Description: Meet minimum combination of programmatic and technical risks and life cycle costs.

B. Evaluation Criterion ID \#: (Note 1)

6.0

C. Evaluation Criterion Weighted

$\mathbf{W}_{1}=$ .12

Value:

D. Utility Functions:

Utility Function (UF) Value $\left(V_{1}\right)=\Sigma$ Level 2 Criterion Weighted Score (WS)

(Note 2)

UF Value Formula: $\quad V_{1}=\underline{W S}_{6.1}+W_{6.2}+W_{6.3}$ (Note 3)

F. Evaluation Criterion weighted score for the Alternative: $\quad W_{1} \times V_{1}=$ Weighted Score $\therefore . . \mathbf{1 2} \times \underline{81.00}=$ Explanatory Notes for Weighted Score:

Notes:

I. For Level I Evaluation Criterion, the ID\# is described by X.0, where $X=1,2,3$,etc. For Level 2 Evaluation Criterion, the $I D \#$ is described by X.I, X.2, X.3, etc. where ' $X$ ' is the Level 1 Evaluation Criterion ID\#.

2. Utility Function values range from 0 (least desirable) to 100 (mast desirable).

3. If Level 2 Criterion are used, the sum of the Level 2 "Weighted Scores" must be multiplied by the Level 1 Weight to determine the Level 1 Weighted Score. 


\section{HLW Salt Disposition Systems Engineering Team} Level 2 Evaluation Criteria Assessment Form

Alternative Number:

6

Alternative Title

Crystalline Silicotitanate (CST) Ion Exchange - DWPF Vitrification

Date: $9 / 18 / 99$

A. Evaluation Criterion

Regulatory Schedule Commitments

Title:

Evaluation Criterion

Description:

Maximize capability of disposing of radioactive wastes per Federal Facility Agreement (FFA) \& Site Treatment Plan (STP) schedules or earlier.

B. Evaluation Criterion ID \#:

(Note 1)

$\underline{6.1}$

C. Evaluation Criterion Weighted

$\mathbf{w}_{2}=$ .5

Value:

D. Utility Functions:

UF Value

(Note 2)

Utility Function (UF) Description:

UF.1 Accelerated Cleanup Plan (ACP) to empty High Level Waste (HLW) tanks by 2022 is met. $\quad 100$

F.2 Base Site Treatment Plan (STP) requirement to close HLW tanks by 2028 is met.

$\underline{70}$

UF.3 Base STP or Federal Facility Agreement (FFA) requirements to close HLW tanks by committed dates $\underline{0}$ is not met.

E.

UF VALUE:

Explanatory Notes for UF Selected:
$V_{2}=$ 80

DWPF operation supports STP requirement. Flexibility to expand throughput, but would not meet $\mathrm{ACP}$

F. Evaluation Criterion weighted score (WS) for the Alternative:

$\mathrm{W}_{2} \times \mathrm{V}_{2}=\mathrm{WS} \therefore \underline{.5} \times \underline{80}=$

40.00

Notes:

1. For Level 1 Evaluation Criterion, the ID\# is described by X.0, where $X=1,2,3$,etc. For Level 2 Evaluation Criterion, the ID\# is described by X.1, X.2, X.3, etc. where ' $X$ ' is the Level 1 Evaluation Criterion ID\#.

2. Utility Function values range from 0 (least desirable) to 100 (most desirable).

3. If Level 2 Criterion are used, the sum of the Level 2 "Weighted Scores" must be multiplied by the Level 1 Weight to determine the Level 1 Weighted Score. 


\section{HLW Salt Disposition Systems Engineering Team \\ Level 2 Evaluation Criteria Assessment Form}

Alternative Number: Alternative Title Crystalline Silicotitanate (CST) Ion Exchange - DWPF Vitrification

Date: 9/18/99

A. Evaluation Criterion Life Cycle Costs (LCC)

Title:

Evaluation Criterion Minimize LCC including TEC, OPC, and D\&D (excludes salvage and repository costs). Description:

B. Evaluation Criterion ID \#: (Note 1)

6.2

C. Evaluation Criterion Weighted

$\mathbf{W}_{2}=$ .3

Value:

D. Utility Functions: UF Value

(Note 2)

Utility Function (UF) Description:

LCC $\leq 2$ billion dollars.

$\underline{\mathrm{LCC}}$ is 4 billion dollars.

$\underline{50}$

UF.3 LCC is 8 billion dollars. 25

UF.4 LCC $\geq 16$ billion dollars. $\underline{0}$

E. UF VALUE: $V_{2}=$ 70

Explanatory Notes for UF Selected: LCC equals 2.9 billion dollars. Based on the point estimate. WSRC-RP-98-00167

F. Evaluation Criterion weighted score (WS) for the Alternative:

$$
\mathrm{W}_{2} \times \mathrm{V}_{2}=\mathrm{WS} \therefore \underline{.3} \times \underline{\mathbf{7 0}}=
$$

21.00

Notes:

1. For Level 1 Evaluation Criterion, the ID\# is described by X.0, where $X=1,2,3$,etc. For Level 2 Evaluation Criterion, the ID\# is described by X.1, X.2, X.3, etc. where ' $X$ ' is the Level 1 Evaluation Criterion ID\#.

2. Utility Function values range from 0 (least desirable) to 100 (most desirable).

3. If Level 2 Criterion are used, the sum of the Level 2 "Weighted Scores" must be multiplied by the Level 1 Weight to determine the Level 1 Weighted Score. 


\section{HLW Salt Disposition Systems Engineering Team} Level 2 Evaluation Criteria Assessment Form

Alternative Number: 6 Alternative Title Crystalline Silicotitanate (CST) Ion Exchange - DWPF Vitrification

Date: $9 / 18 / 99$

A. Evaluation Criterion Repository Costs

Title:

Evaluation Criterion Description: Minimize cost for waste disposal off-site (Federal Repository).

B. Evaluation Criterion ID \#: (Note 1)

6.3

$$
\text { (Note 1) }
$$

C. Evaluation Criterion Weighted

Value:

$\mathbf{W}_{2}=$ 2

D. Utility Functions:

Utility Function (UF) Description:

UF Value

(Note 2)

$\underline{100}$

$\underline{50}$

$\underline{0}$

UF.3 $\geqq 130,000$ canisters for off-site disposal

E. UF VALUE:

$\mathbf{V}_{2}=$ 100

Explanatory Notes for UF Selected:

DWPF canister production remains at 6000

F. Evaluation Criterion weighted score (WS) for the Alternative:

$$
\mathbf{W}_{2} \times \mathbf{V}_{2}=\mathbf{W S} \therefore .2 \times \underline{100}=\underline{20.00}
$$

Notes:

1. For Level 1 Evaluation Criterion, the ID\# is described by X.0, where $X=1,2,3$, etc. For Level 2 Evaluation Criterion, the ID\# is described by X.1, X.2, X.3, etc. where ' $X$ ' is the Level I Evaluation Criterion ID\#.

2. Utility Function values range from 0 (least desirable) to 100 (most desirable).

3. If Level 2 Criterion are used, the sum of the Level 2 "Weighted Scores" must be multiplied by the Level 1 Weight to determine the Level 1 Weighted Score. 
High Level Waste Salt Disposition

WSRC-RP-99-00007

Systems Engineering Team

Revision: 0

Final Report

Page 95 of 277

\subsubsection{Direct Disposal in Grout}




\section{HLW Salt Disposition Systems Engineering Team Level 1 Evaluation Criteria Assessment Form}

Alternative Number:

Alternative Title Direct Disposal to Grout

Date: $9 / 18 / 99$

A. Evaluation Criterion

Technology

Title:

Evaluation Criterion

Description:

Maximize the confidence that underlying scientific principles \& engineering implementation will result in adequate attainment.

B. Evaluation Criterion ID \#:

(Note 1)

1.0

C. Evaluation Criterion Weighted

$\mathbf{W}_{1}=$

.23

Value:

D. Utility Functions:

Utility Function (UF) Value $\left(V_{1}\right)=\Sigma$ Level 2 Criterion Weighted Score (WS)

(Note 2)

UF Value Formula: $\quad \mathbf{V}_{1}=\underline{W S}_{1.1}+\mathbf{W S}_{1.2}+\mathbf{W S}_{1.3}$

(Note 3)

F. Evaluation Criterion weighted score for the Alternative: $\quad W_{1} \times V_{1}=$ Weighted Score $\therefore . .23 \times \underline{86.00}=$

Explanatory Notes for Weighted Score:

Notes:

1. For Level 1 Evaluation Criterion, the ID\# is described by X.O, where $X=1,2,3$, etc. For Level 2 Evaluation Criterion, the $I D \#$ is described by $X .1, X .2, X .3$, etc. where ' $X$ ' is the Level 1 Evaluation Criterion ID\#.

2. Utility Function values range from 0 (least desirable) to 100 (most desirable).

3. If Level 2 Criterion are used, the sum of the Level 2 "Weighted Scores" must be multiplied by the Level 1 Weight to determinethe Level 1 Weighted Score. 


\section{HLW Salt Disposition Systems Engineering Team}

Level 2 Evaluation Criteria Assessment Form

Alternative Number: Alternative Title Direct Disposal to Grout

Date: $9 / 18 / 99$

A. Evaluation Criterion Scientific Maturity

Title: Evaluation Criterion Description: The level of scientific understanding needed to minimize project risk.

B. Evaluation Criterion ID \#: (Note 1)

C. Evaluation Criterion Weighted

$$
\mathbf{W}_{2}=
$$
.4

Value:

D. Utility Functions:

Utility Function (UF) Description:

(Note 2)

Reliable radioactive production scale demonstration \& correlation to predicted scientific results. $\quad \underline{100}$

JF.2 Large scale radioactive test: 'spiked' radiochemistry demonstration.

UF.3 Pilot (small) scale radioactive test; full radiochemistry.

UF.4 Lab scale test; simulant/real waste.

UF.5 Theoretical understanding only; no practical demonstration.

E.

UF VALUE:

Explanatory Notes for UF Selected:
Grout formulation changes to address the potassium and cesium difference from the existing Saltstone process.

F. Evaluation Criterion weighted score (WS) for the Alternative:

$$
\mathrm{W}_{2} \times \mathrm{V}_{2}=\mathrm{WS} \therefore \underline{.4} \times \underline{\mathbf{9 5}}=
$$
38.00

Notes:

1. For Level 1 Evaluation Criterion, the ID\# is described by X.O, where $X=1,2,3$,etc. For Level 2 Evaluation Criterion, the ID\# is described by X.1,X.2,X.3, etc. where ' $X$ ' is the Level 1 Evaluation Criterion ID\#.

2. Utility Function values range from 0 (least desirable) to 100 (most desirable).

3. If Level 2 Criterion are used, the sum of the Level 2 "Weighted Scores" must be multiplied by the Level 1 Weight to determine the Level 1 Weighted Score. 


\section{HLW Salt Disposition Systems Engineering Team Level 2 Evaluation Criteria Assessment Form}

Alternative Number: 18 Alternative Title Direct Disposal to Grout

Date: 9/18/99

A. Evaluation Criterion Engineering Maturity

Title:

Evaluation Criterion

The level of applied engineering concepts needed to minimize project risk

Description:

B. Evaluation Criterion ID \#:

(Note 1)

C. Evaluation Criterion Weighted

$\mathbf{W}_{2}=$ .4

Value:

D. Utility Functions:

UF Value

(Note 2)

Utility Function (UF) Description:

UF.1 Reliable radioactive production scale with significant operating experience.

UF.2 Reliable non-radioactive production scale with significant operating experience.

UF.3 Limited radioactive production scale.

UF.4 Limited non-radioactive production scale

$\underline{20}$

UF.5 Demonstration

E.

UF VALUE:

Explanatory Notes for UF Selected:

SRS Saltstone, BNFL Sellafield, West Valley, and Oak Ridge experience. Alpha removal process provides some engineering challenges in the areas of filtration, mixing and pumping. WSRC-TR-99-00342; WSRC-RP-99-0006.

F. Evaluation Criterion weighted score (WS) for the Alternative:

$$
W_{2} \times V_{2}=W S \therefore .4 \times \underline{.40}=
$$

28.00

Notes:

1. For Level 1 Evaluation Criterion, the ID\# is described by X.O, where $X=1,2,3$,etc. For Level 2 Evaluation Criterion, the $I D \#$ is described by X.I, X.2, X.3, etc. where ' $X$ ' is the Level I Evaluation Criterion ID\#.

2. Utility Function values range from 0 (least desirable) to 100 (most desirable).

3. If Level 2 Criterion are used, the sum of the Level 2 "Weighted Scores" must be multiplied by the Level 1 Weight to determine the Level I Weighted Score. 


\section{HLW Salt Disposition Systems Engineering Team}

Level 2 Evaluation Criteria Assessment Form

Alternative Number: 18 Alternative Title Direct Disposal to Grout

Date: 9/18/99

A. Evaluation Criterion Process Simplicity

Title:

Evaluation Criterion

Description: Ease of Science implementation understanding by operators.

B. Evaluation Criterion ID \#: (Note 1)

C. Evaluation Criterion Weighted

Value:

$\mathbf{W}_{2}=$ .2

D. Utility Functions:

UF Value

(Note 2)

Utility Function (UF) Description:

Low complexity, straight forward operations.

$\underline{100}$

$\underline{70}$

Moderate complexity - operator aids and routine engineering support.

$\underline{0.0}$

UF.3 Complex - significant training for operators and continuous, specialized engineering support required.

E. UF VALUE:

$$
\mathbf{V}_{2}=
$$
100

Explanatory Notes for UF Selected:

Intrinsic process simplicity and much operating experience.

F. Evaluation Criterion weighted score (WS) for the Alternative:

$$
\mathrm{W}_{2} \times \mathrm{V}_{2}=\mathrm{WS} \therefore \underline{.2} \times \underline{100}=\underline{20.00}
$$

Notes:

1. For Level 1 Evaluation Criterion, the ID\# is described by X.O, where $X=1,2,3$, etc. For Level 2 Evaluation Criterion, the $I D \#$ is described by X.I, X.2, X.3, etc. where ' $X$ ' is the Level I Evaluation Criterion ID\#.

2. Utility Function values range from 0 (least desirable) to 100 (most desirable).

3. If Level 2 Criterion are used, the sum of the Level 2 "Weighted Scores" must be multiplied by the Level 1 Weight to determine the Level I Weighted Score. 


\section{HLW Salt Disposition Systems Engineering Team} Level 1 Evaluation Criteria Assessment Form

Alternative Number: 18 Alternative Title Direct Disposal to Grout

Date: 9/18/99

A. Evaluation Criterion Current Mission Interfaces

Title:

Evaluation Criterion Impact on current SRS missions/programs.

Description:

B. Evaluation Criterion ID \#: 2.0 (Note 1)

C. Evaluation Criterion Weighted Value:

$W_{1}=$ .15

D. Utility Functions:

Utility Function (UF) Value $\left(\mathrm{V}_{\mathrm{l}}\right)=\Sigma$ Level 2 Criterion Weighted Score (WS)

(Note 2)

UF Value Formula: (Note 3)

$V_{1}=W S_{2.1}+W S_{2.2}+W S_{2.3}+W S_{2.4}+W S_{2.5}$

F. Evaluation Criterion weighted score for the Alternative:

$\mathbf{W}_{1} \times \mathrm{V}_{1}=$ Weighted Score

.15

$15 \times \underline{78.25}=$

Explanatory Notes for Weighted Score:

Notes:

1. For Level 1 Evaluation Criterion, the ID\# is described by $X .0$, where $X=1,2,3$,etc. For Level 2 Evaluation Criterion, the $I D \#$ is described by X.1, X.2, X.3, etc. where ' $X$ ' is the Level 1 Evaluation Criterion ID\#.

2. Utility Function values range from 0 (least desirable) to 100 (most desirable).

3. If Level 2 Criterion are used, the sum of the Level 2 "Weighted Scores" must be multiplied by the Level 1 Weight to determine the Level I Weighted Score. 


\section{HLW Salt Disposition Systems Engineering Team}

Level 2 Evaluation Criteria Assessment Form

Alternative Number: 18 Alternative Title Direct Disposal to Grout

Date: $9 / 18 / 99$

A. Evaluation Criterion

DWPF

Title:

Evaluation Criterion

Impact on DWPF (Table 1 Functions \& Requirements).

Description:

B. Evaluation Criterion ID \#:

(Note 1)

$\underline{2.1}$

C. Evaluation Criterion Weighted

$\mathbf{W}_{2}=$ .25

Value:

D. Utility Functions:

UF Value

(Note 2)

Utility Function (UF) Description:

UF.1 Sludge only to completion

100

JF.2 Sludge plus MST to completion.

$\underline{85}$

UF.3 Baseline - current ITP flowsheet.

$\underline{70}$

UF.4 Moderate impact - some additional canisters $(<50 \%)$. Facility modifications required.

$\underline{20}$

UF.5 Significant impact - additional canisters $(>50 \%)$ glass reformulation/repermitting required. Major facility modifications required.

E.

UF VALUE:

$\mathbf{V}_{2}=$

85

F. Evaluation Criterion weighted score (WS) for the Alternative:

$$
W_{2} \times V_{2}=W S \therefore .25 \times \underline{85}=\underline{21.25}
$$

Notes:

1. For Level 1 Evaluation Criterion, the ID\# is described by X.0, where $X=1,2,3$,etc. For Level 2 Evaluation Criterion, the $I D \#$ is described by X.1, X.2, X.3, etc. where ' $X$ ' is the Level 1 Evaluation Criterion ID\#.

2. Utility Function values range from 0 (least desirable) to 100 (most desirable).

3. If Level 2 Criterion are used, the sum of the Level 2 "Weighted Scores" must be multiplied by the Level 1 Weight to determine the Level 1 Weighted Score. 


\section{HLW Salt Disposition Systems Engineering Team Level 2 Evaluation Criteria Assessment Form}

Alternative Number: 18 Alternative Title Direct Disposal to Grout

Date: $9 / 18 / 99$

A. Evaluation Criterion

Title:

Evaluation Criterion

Saltstone

Description:

Impact on Saltstone (Table 1 Functions \& Requirements)

B. Evaluation Criterion ID \#:

(Note 1)

C. Evaluation Criterion Weighted

$W_{2}=$ .15

Value:

D. Utility Functions:

UF Value

(Note 2)

Utility Function (UF) Description:

UF.1 No need for Saltstone Facility.

UF.3 180M gallons saltstone plus Benzene risk (current flowsheet).

$\underline{70}$

UF.4 Moderate increase in saltstone $(<50 \%)$. Minor facility modifications.

UF.5 Repermit saltstone to Class C waste. Major facility modifications and increased throughtput $(>50 \%)$.

E. UF VALUE:

Explanatory Notes for UF Selected:

F. Evaluation Criterion weighted score (WS) for the Alternative:
$\mathbf{V}_{2}=$ 100

New facility eliminates the need for the existing Saltstone facility (results in retirement)

Notes:

1. For Level I Evaluation Criterion, the ID\# is described by X.O, where $X=1,2,3$, etc. For Level 2 Evaluation Criterion, the ID\# is described by X.I, X.2, X.3, etc. where ' $X$ ' is the Level I Evaluation Criterion ID\#.

2. Utility Function values range from 0 (least desirable) to 100 (most desirable).

3. If Level 2 Criterion are used, the sum of the Level 2 "Weighted Scores" must be multiplied by the Level 1 Weight to determine the Level 1 Weighted Score. 
HLW Salt Disposition Systems Engineering Team

Level 2 Evaluation Criteria Assessment Form

Alternative Number: 18 Alternative Title Direct Disposal to Grout

Date: $9 / 18 / 99$

A. Evaluation Criterion Solid Waste

Title:

Evaluation Criterion Impact on Solid Waste (Table 1 Functions \& Requirements).

Description:

B. Evaluation Criterion ID \#: (Note 1)

C. Evaluation Criterion Weighted

$\mathbf{W}_{2}=$ .1 Value:

D. Utility Functions:

UF Value

(Note 2)

Utility Function (UF) Description:

UF.1 Reduced solid waste volume and no Benzene.

Reduced solid waste volume and Benzene.

$\underline{80}$

UF.3 Current flowsheet (Benzene to CIF).

$\underline{50}$

UF.4 Moderate increase in solid waste volume.

$\underline{30}$

UF.5 Repermit new waste forms, significant increase in solid waste volume.
E.

\section{UF VALUE:}

Explanatory Notes for UF Selected:

$$
\mathbf{V}_{2}=
$$
80

No increase in equipment or job control waste to be handled by Solid Waste Division vaults (excluding saltstone grout). Reduction in liquid benzene generation by 35,000 gallons per year (no benzene generated).

F. Evaluation Criterion weighted score (WS) for the Alternative:

$$
\mathrm{W}_{2} \times \mathrm{V}_{2}=\mathrm{WS} \therefore . \mathbf{1} \times \underline{80}=
$$
8.00

Notes:

1. For Level 1 Evaluation Criterion, the ID\# is described by X.O, where $X=1,2,3$,etc. For Level 2 Evaluation Criterion, the $I D \#$ is described by X.I, X.2, X.3, etc. where ' $X$ ' is the Level 1 Evaluation Criterion ID\#.

2. Utility Function values range from 0 (least desirable) to 100 (most desirable).

3. If Level 2 Criterion are used, the sum of the Level 2 "Weighted Scores" must be multiplied by the Level 1 Weight to determine the Level I Weighted Score. 


\section{HLW Salt Disposition Systems Engineering Team} Level 2 Evaluation Criteria Assessment Form

Alternative Number: 18 Alternative Title Direct Disposal to Grout

Date: $9 / 18 / 99$

A.

Evaluation Criterion

Tank Farm

Title:

Evaluation Criterion Impact on Tank Farm (Table 1 Functions \& Requirements)

Description:

B. Evaluation Criterion ID \#: (Note 1)

C. Evaluation Criterion Weighted

$\mathbf{W}_{2}=$ .2 Value:

D. Utility Functions:

UF Value

(Note 2)

Utility Function (UF) Description: impact.

UF.2 Current flowsheet.

$\underline{50}$

UF.3 Increased safety hazards (e.g. Organics) increase operational capacity, increased corrosion impacts. $\underline{0}$

E.

UF VALUE:

Explanatory Notes for UF Selected:
$\mathbf{V}_{2}=$ 80

Based on not operating a precipitate process through DWPF there is no benzene or cesium in the recycle and a reduction in recycle water volume and subsequent impact on corrosion control. Reduced evaporator operations. WSRC-RP-98-00168, R1

F. Evaluation Criterion weighted score (WS) for the Alternative:

$$
W_{2} \times V_{2}=W S \therefore .2 \times .80=
$$
16.00

Notes:

1. For Level I Evaluation Criterion, the ID\# is described by X.0, where $X=1,2,3$,etc. For Level 2 Evaluation Criterion, the ID\# is described by X.1, X.2, X.3, etc. where ' $X$ ' is the Level 1 Evaluation Criterion ID\#.

2. Utility Function values range from 0 (least desirable) to 100 (most desirable).

3. If Level 2 Criterion are used, the sum of the Level 2 "Weighted Scores" must be multiplied by the Level 1 Weight to determine the Level 1 Weighted Score. 


\section{HLW Salt Disposition Systems Engineering Team} Level 2 Evaluation Criteria Assessment Form

Alternative Number: 18

Alternative Title Direct Disposal to Grout

Date: 9/18/99

A. Evaluation Criterion

Tank Farm Space Management

Title:

Evaluation Criterion

Description:

Utilization of available Tank Farm storage \& resources as a function of time (HLW Salt Disposition Interface Functional Performance Requirement).

B. Evaluation Criterion ID \#: (Note 1)

$\underline{2.5}$

C. Evaluation Criterion Weighted

Value:

$$
W_{2}=
$$

D. Utility Functions:

UF Value

(Note 2)

Utility Function (UF) Description:

Accelerate tank space gain. Tank space adequate for current and future missions.

$\underline{100}$

Current flowsheet (reduces available tank space)

UF.3 Accelerated reduction in available tank space (water logged tank farm).

$\underline{0}$
E.

UF VALUE:

Explanatory Notes for UF Selected:

DWPF recycle is reduced by 500,000 gallons per year due to no precipitate hydrolysis process. Salt solution work off rate is $17.5 \mathrm{gpm}$. TK49 readily available for waste storage. TK48 available after waste handling strategy is completed. WSRC-RP-98-00168, R1; WSRC-RP-99-00005

F. Evaluation Criterion weighted score (WS) for the Alternative:

$\mathrm{W}_{2} \times \mathrm{V}_{2}=\mathrm{WS} \therefore \underline{.3} \times \underline{60}=$

18.00

Notes:

1. For Level 1 Evaluation Criterion, the ID\# is described by X.0, where $X=1,2,3$,etc. For Level 2 Evaluation Criterion, the ID\# is described by X.1, X.2, X.3, etc. where ' $X$ ' is the Level I Evaluation Criterion ID\#.

2. Utility Function values range from 0 (least desirable) to 100 (most desirable).

3. If Level 2 Criterion are used, the sum of the Level 2 "Weighted Scores" must be multiplied by the Level 1 Weight to determine the Level 1 Weighted Score. 


\section{HLW Salt Disposition Systems Engineering Team Level 1 Evaluation Criteria Assessment Form}

Alternative Number: 18

Alternative Title Direct Disposal to Grout

Date: $9 / 18 / 99$

A. Evaluation Criterion Future Mission Interfaces

Title:

Evaluation Criterion Maximize the support of identified potential future missions.

Description:

B. Evaluation Criterion ID \#: 3.0 (Note 1)

C. Evaluation Criterion Weighted

$\mathbf{W}_{1}=$ .07

Value:

D. Utility Functions:

UF Value

(Note 2)

Utility Function (UF) Description:

UF.1 Flexible system capable of supporting identified potential future missions.

100

System will support can-in-can and spent fuel stabilization.

$\underline{70}$

UF.3 System will not support can-in-can or spent fuel stabilization.

$\underline{0}$
E.

UF VALUE:

Explanatory Notes for UF Selected:
$V_{1}=$ 35

Based on technical viability to support spent fuel stabilization. Does not support can-incan mission because cesium does not go to DWPF.

F. Evaluation Criterion weighted score (WS) for the Alternative

$$
\mathrm{W}_{1} \times \mathrm{V}_{1}=\mathrm{WS} \therefore \underline{.07} \times \underline{35}=\underline{2.45}
$$

Notes:

1. For Level 1 Evaluation Criterion, the ID\# is described by $X .0$, where $X=1,2,3$,etc. For Level 2 Evaluation Criterion, the ID\# is described by X.I, X.2, X.3, etc. where ' $X$ ' is the Level 1 Evaluation Criterion ID\#.

2. Utility Function values range from 0 (least desirable) to 100 (most desirable).

3. If Level 2 Criterion are used, the sum of the Level 2 "Weighted Scores" must be multiplied by the Level 1 Weight to determine the Level 1 Weighted Score. 
HLW Salt Disposition Systems Engineering Team Level 1 Evaluation Criteria Assessment Form

Alternative Number: 18 Alternative Title Direct Disposal to Grout

Date: $9 / 18 / 99$

A. Evaluation Criterion Regulatory/ISMS/Environmental

Title:

Evaluation Criterion Description:

Protect personnel \& the environment from hazards \& releases of waste \& pollution by ensuring maximum application of intrinsic safety features.

B. Evaluation Criterion ID \#: (Note 1)

4.0

C. Evaluation Criterion Weighted Value:

$W_{1}=$ .23

D. Utility Functions:

Utility Function (UF) Value $\left(\mathrm{V}_{1}\right)=\Sigma$ Level 2 Criterion Weighted Score (WS)

(Note 2)

E. UF Value Formula: $\quad \mathbf{V}_{1}=\mathbf{W S}_{4.1}+\mathbf{W S}_{4.2}+\mathbf{W S}_{4.3}$ (Note 3)

F. Evaluation Criterion weighted score for the Alternative: $\quad W_{1} \times V_{1}=$ Weighted Score $\therefore .23 \times \underline{49.00}=$ 11.27 Explanatory Notes for Weighted Score:

Notes:

1. For Level 1 Evaluation Criterion, the ID\# is described by X.O, where $X=1,2$, 3, etc. For Level 2 Evaluation Criterion, the $I D \#$ is described by $X .1, X .2, X .3$, etc. where ' $X$ ' is the Level I Evaluation Criterion ID\#.

2. Utility Function values range from 0 (least desirable) to 100 (most desirable).

3. If Level 2 Criterion are used, the sum of the Level 2 "Weighted Scores" must be multiplied by the Level 1 Weight to determine the Level I Weighted Score. 


\section{HLW Salt Disposition Systems Engineering Team}

Level 2 Evaluation Criteria Assessment Form

Alternative Number: 18 Alternative Title Direct Disposal to Grout

Date: $9 / 18 / 99$

A. Evaluation Criterion

Public/Environment

Title:

Evaluation Criterion Description:

Protect the public \& environment from hazards \& accidental releases of waste \& pollution by ensuring maximum application of intrinsic safety features.

B. Evaluation Criterion ID \#:

(Note 1)

$\underline{4.1}$

C. Evaluation Criterion Weighted

$\mathbf{W}_{2}=$ .45

Value:

D. Utility Functions:

UF Value

(Note 2)

Utility Function (UF) Description:

UF.1 Process is inherently safe and can be quantified/documented in Authorization Basis.

JF.2 Process has moderate hazards that are passively mitigated.

$\underline{85}$

UF.3 Process has moderate hazards that are readily mitigated.

UF.4 Process has inherent hazards that can be mitigated with Engineered Safety Features and

$\underline{35}$

Administrative Controls.

UF.5 Process has inherent hazards and the risks are not quantifiable.

$\underline{0}$

E.

UF VALUE:

$\mathbf{V}_{2}=$ 60

Explanatory Notes for UF Selected: $\quad$ Hydrogen source in alpha removal tank provides energy for source term dispersion. WSRC-RP-98-00168, R1; S-CLC-G-00187

F. Evaluation Criterion weighted score (WS) for the Alternative:

$\mathrm{W}_{2} \times \mathrm{V}_{2}=\mathrm{WS} \therefore \underline{.45} \times \underline{60}=\underline{27.00}$

Notes:

1. For Level I Evaluation Criterion, the ID\# is described by X.0, where $X=1,2,3$, etc. For Level 2 Evaluation Criterion, the ID\# is described by X.1, X.2, X.3, etc. where ' $X$ ' is the Level I Evaluation Criterion ID\#.

2. Utility Function values range from 0 (least desirable) to 100 (most desirable).

3. If Level 2 Criterion are used, the sum of the Level 2 "Weighted Scores" must be multiplied by the Level 1 Weight to determine the Level 1 Weighted Score. 


\section{HLW Salt Disposition Systems Engineering Team \\ Level 2 Evaluation Criteria Assessment Form}

Alternative Number: 18 Alternative Title Direct Disposal to Grout

Date: $9 / 18 / 99$

A. Evaluation Criterion

Title:

Evaluation Criterion

Description:

Worker

Protect on-site personnel from hazards \& accidental releases of waste \& pollution by ensuring maximum application of intrinsic safety features.

B. Evaluation Criterion ID \#:

(Note 1)

4.2

C. Evaluation Criterion Weighted

$\mathbf{W}_{2}=$ .35

Value:

D. Utility Functions:

UF Value

(Note 2)

Utility Function (UF) Description:

UF.1 Process is inherently safe and poses no unusual worker safety hazard.

UF.2 Process has moderate hazards that are passively mitigated.

UF.3 Process has moderate hazards that are readily mitigated.

$\underline{40}$

UF.4 Process has inherent hazards that can be mitigated with Structures, Systems, Components and Administrative Controls.

UF.5 Process has inherent hazards and poses significant risk to worker safety that are not readily mitigated. $\underline{0}$

E.

UF VALUE:

$\mathbf{V}_{2}=$ 60

Explanatory Notes for UF Selected:

Hydrogen source in alpha removal tank provides energy for source term dispersion. WSRC-RP-98-00168, R1: S-CLC-G-00187.

F. Evaluation Criterion weighted score (WS) for the Alternative:

$\mathrm{W}_{2} \times \mathrm{V}_{2}=\mathrm{WS} \therefore \underline{.35} \times \underline{60}=$ 21.00

Notes:

1. For Level 1 Evaluation Criterion, the ID\# is described by $X .0$, where $X=1,2,3$,etc. For Level 2 Evaluation Criterion, the ID\# is described by X.1, X.2, X.3, etc. where ' $X$ ' is the Level I Evaluation Criterion ID\#.

2. Utility Function values range from 0 (least desirable) to 100 (most desirable).

3. If Level 2 Criterion are used, the sum of the Level 2 "Weighted Scores" must be multiplied by the Level I Weight to determine the Level 1 Weighted Score. 


\section{HLW Salt Disposition Systems Engineering Team} Level 2 Evaluation Criteria Assessment Form

Alternative Number: 18 Alternative Title Direct Disposal to Grout

Date: $9 / 18 / 99$

A. Evaluation Criterion

Permitting

Title:

Evaluation Criterion

Minimize waste generation risk \& difficulty of permitting new releases \& waste forms.

Description:

B. Evaluation Criterion ID \#: (Note 1)

$\underline{4.3}$

C. Evaluation Criterion Weighted

$\mathbf{W}_{2}=$ .2

Value:

D. Utility Functions:

UF Value

Utility Function (UF) Description:

(Note 2)

UF.1 No new waste forms requiring permitting, eliminate one or more existing releases, no requalification

UF.1 No new waste forms requiring permitting, eliminate one or more existing releases, no requalification

UF.2 Reduction in current releases, no additional permitting required.

$\underline{100}$

$\underline{80}$

UF.3 Current flowsheet (Saltstone Facility needs repermitting due to Benzene releases).

$\underline{60}$

UF.4 Requalification of existing waste form, exceeds current release levels.

UF.5 New waste form permit required, significant increase in environmental releases requiring

$\underline{0}$ repermitting, high level waste retained in South Carolina

E. UF VALUE:

Explanatory Notes for UF Selected:

$$
V_{2}=5
$$

Grout considered new waste form of high level waste retained in South Carolina. Eliminates benzene releases.

F. Evaluation Criterion weighted score (WS) for the Alternative:

$\mathbf{W}_{2} \times \mathbf{V}_{2}=\mathbf{W S} \therefore \underline{.2} \times \underline{5}=$ 1.00

Notes:

1. For Level 1 Evaluation Criterion, the ID\# is described by $X .0$, where $X=1,2,3$,etc. For Level 2 Evaluation Criterion, the $I D \#$ is described by X.1, X.2, X.3, etc. where ' $X$ ' is the Level I Evaluation Criterion ID\#.

2. Utility Function values range from 0 (least desirable) to 100 (most desirable).

3. If Level 2 Criterion are used, the sum of the Level 2 "Weighted Scores" must be multiplied by the Level 1 Weight to determine the Level 1 Weighted Score. 


\section{HLW Salt Disposition Systems Engineering Team} Level 1 Evaluation Criteria Assessment Form

Alternative Number: 18 Alternative Title Direct Disposal to Grout

Date: 9/18/99

A. Evaluation Criterion Engineering (Design)

Title:

Evaluation Criterion Description: Maximize the confidence that the facility meets applicable codes, standards \& required production throughput.

B. Evaluation Criterion ID \#: (Note 1)

$\underline{5.0}$

C. Evaluation Criterion Weighted

$W_{1}=$ .2 Value:

D. Utility Functions:

Utility Function (UF) Value $\left(V_{1}\right)=\Sigma$ Level 2 Criterion Weighted Score (WS)

(Note 2)

UF Value Formula: $\quad \mathbf{V}_{1}=\mathbf{W S}_{5.1}+\mathbf{W S}_{\underline{5.2}}+\mathbf{W S}_{\underline{5.3}}+\mathbf{W S}_{5.4}$ (Note 3)

F. Evaluation Criterion weighted score for the Alternative: $\quad W_{1} \times V_{1}=$ Weighted Score $\therefore . .2 \times \underline{92.50}=$ 18.50 Explanatory Notes for Weighted Score:

Notes:

1. For Level 1 Evaluation Criterion, the ID\# is described by X.0, where $X=1,2,3$,etc. For Level 2 Evaluation Criterion, the ID\# is described by X.1, X.2, X.3, etc. where ' $X$ ' is the Level 1 Evaluation Criterion ID\#.

2. Utility Function values range from 0 (least desirable) to 100 (most desirable).

3. If Level 2 Criterion are used, the sum of the Level 2 "Weighted Scores" must be multiplied by the Level 1 Weight to determine the Level 1 Weighted Score. 


\section{HLW Salt Disposition Systems Engineering Team} Level 2 Evaluation Criteria Assessment Form

Alternative Number: 18 Alternative Title Direct Disposal to Grout

Date: $9 / 18 / 99$

A. Evaluation Criterion

Title:

Evaluation Criterion Description:
Construct

Ensure facility design considers major construction/testing methods and needs in accordance with Integrated Work Process (IWP) and Key Activities for Successful Execution (KASE).

B. Evaluation Criterion ID \#:

(Note 1)

5.1

C. Evaluation Criterion Weighted

$\mathbf{W}_{2}=$ .25

Value:

D. Utility Functions:

UF Value

(Note 2)

Utility Function (UF) Description:

$\underline{100}$

Facility design features and construction methods lead to simplicity of construction/testing process.

$\underline{60}$ complexity).

UF.3 Facility design features and construction methods are difficult to apply due to non-standard, non- $\quad \underline{0}$ commercial methods not readily applied in radioactive environment.

E.

UF VALUE:

$\mathbf{V}_{2}=$ 100

Explanatory Notes for UF Selected: Straight forward process currently in use at SRS.

F. Evaluation Criterion weighted score (WS) for the Alternative

$\mathrm{W}_{2} \times \mathrm{V}_{2}=\mathrm{WS} \therefore \underline{.25} \times \underline{100}=$ $\underline{25.00}$

Notes:

1. For Level 1 Evaluation Criterion, the ID\# is described by X.0, where $X=1,2,3$,etc. For Level 2 Evaluation Criterion, the ID\# is described by X.1, X.2, X.3, etc. where ' $X$ ' is the Level 1 Evaluation Criterion ID\#.

2. Utility Function values range from 0 (least desirable) to 100 (most desirable).

3. If Level 2 Criterion are used, the sum of the Level 2 "Weighted Scores" must be multiplied by the Level 1 Weight to determine the Level 1 Weighted Score. 


\section{HLW Salt Disposition Systems Engineering Team Level 2 Evaluation Criteria Assessment Form}

Alternative Number: 18 Alternative Title Direct Disposal to Grout

Date: $9 / 18 / 99$

A. Evaluation Criterion

Qualify

Title:

Evaluation Criterion

Description:

Readily validate defined functional design requirements, regulatory requirements, final disposal forms, and Authorization Basis (AB) safety requirements.

B. Evaluation Criterion ID \#:

(Note 1)

C. Evaluation Criterion Weighted

$\mathrm{W}_{2}=$ .25

Value:

D. Utility Functions:

Utility Function (UF) Description:

Test program has known acceptance criteria and accomodates direct verification of design attributes. $\quad 100$

UF.2 Test program applies "Graded Approach" to verify key design attributes with other limited testing $\quad \underline{70}$ and inferred results.

UF.3 Insufficient science/engineering exists to establish firm test acceptance criteria and methods, limited $\quad \underline{0}$ direct verification.

E.

UF VALUE:

$\mathbf{V}_{2}=$ 90

Explanatory Notes for UF Selected: Grout analysis not specified to support verification of grout formulation.

F. Evaluation Criterion weighted score (WS) for the Alternative:

$\mathrm{W}_{2} \times \mathrm{V}_{2}=\mathrm{WS} \therefore .25 \times \underline{.20}=\underline{22.50}$

Notes:

1. For Level 1 Evaluation Criterion, the ID\# is described by X.O, where $X=1,2,3$,etc. For Level 2 Evaluation Criterion, the $I D \#$ is described by X.I, X.2, X.3, etc. where ' $X$ ' is the Level 1 Evaluation Criterion ID\#.

2. Utility Function values range from 0 (least desirable) to 100 (most desirable).

3. If Level 2 Criterion are used, the sum of the Level 2 "Weighted Scores" must be multiplied by the Level 1 Weight to determine the Level 1 Weighted Score. 


\section{HLW Salt Disposition Systems Engineering Team Level 2 Evaluation Criteria Assessment Form}

Alternative Number: 18

Alternative Title Direct Disposal to Grout

Date: $9 / 18 / 99$

A. Evaluation Criterion

Title:

Evaluation Criterion

Description:

Operate

Maximize ease of repeat operation/proceduralization, access for round sheets/physical verification, and upset operation management (Section R-1.4-3 of Functions \& Requirements).

B. Evaluation Criterion ID \#: (Note 1)

C. Evaluation Criterion Weighted

$\mathrm{W}_{2}=$ .25 Value:

D. Utility Functions:

UF Value

(Note 2)

Utility Function (UF) Description:

Design allows simple, coordinated, straight forward operation with direct access to key controls, interlocks, and instruments. Easy access to key equipment, maximize ALARA considerations. Minimize number of process control points.

UF.2 Design allows manageable operation with minimal complexity (Standard SRS practice).

$\underline{100}$

$\underline{60}$

UF.3 Design is highly coupled with minimum holdup, multiple parallel operations and fast dynamics, and process instability.

E.

UF VALUE:

$\mathbf{V}_{2}=$ 100

Explanatory Notes for UF Selected: Simple straight forward process.

F. Evaluation Criterion weighted score (WS) for the Alternative: $\quad \mathbf{W}_{2} \times \mathrm{V}_{2}=\mathbf{W S} \therefore \underline{.25} \times \underline{100}=\underline{\mathbf{2 5 . 0 0}}$

Notes:

1. For Level 1 Evaluation Criterion, the ID\# is described by X.O, where $X=1,2,3$,etc. For Level 2 Evaluation Criterion, the $I D \#$ is described by X.1, X.2, X.3, etc. where ' $X$ ' is the Level 1 Evaluation Criterion ID\#.

2. Utility Function values range from 0 (least desirable) to 100 (most desirable).

3. If Level 2 Criterion are used, the sum of the Level 2 "Weighted Scores" must be multiplied by the Level I Weight to determine the Level 1 Weighted Score. 


\section{HLW Salt Disposition Systems Engineering Team Level 2 Evaluation Criteria Assessment Form}

Alternative Number: 18 Alternative Title Direct Disposal to Grout

Date: $9 / 18 / 99$

A. Evaluation Criterion RAMI

Title:

Evaluation Criterion Design to maximize Reliability, Availability, Maintainability, and Inspectability.

Description:

B. Evaluation Criterion ID \#: $\quad 5.4$ (Note 1)

C. Evaluation Criterion Weighted

$\mathbf{W}_{2}=$ .25

Value:

D. Utility Functions:

UF Value

(Note 2)

Utility Function (UF) Description:

UF.1 Design allows simple, coordinated, straight forward maintenance practices which take into account ALARA requirements. Design maximizes reliability, and availability of Structures, Systems, and Components.

UF.2 Design allows manageable maintenance functions with minimal complexibility (Standard SRS practice).

UF.3 Design complexity restricts maintainability and inspectability and reduces reliability, availability of Structures, Systems, and Components. Remotability restricts maintainability.

E.

UF VALUE:

Explanatory Notes for UF Selected:

$$
\mathbf{V}_{2}=
$$
80

Minimal equipment with "in-canyon" service. Simple unit operations. Alpha removal equipment size adds RAMI complexity. WSRC-RP-99-006

F. Evaluation Criterion weighted score (WS) for the Alternative:

$\mathrm{W}_{2} \times \mathrm{V}_{2}=\mathrm{WS} \therefore . .25 \times \underline{80}=\underline{20.00}$

Notes:

1. For Level I Evaluation Criterion, the ID\# is described by X.0, where $X=1,2,3$,etc. For Level 2 Evaluation Criterion, the $I D \#$ is described by $X .1, X .2, X .3$, etc. where ' $X$ ' is the Level 1 Evaluation Criterion ID\#.

2. Utility Function values range from 0 (least desirable) to 100 (most desirable).

3. If Level 2 Criterion are used, the sum of the Level 2 "Weighted Scores" must be multiplied by the Level 1 Weight to determine the Level I Weighted Score. 


\section{HLW Salt Disposition Systems Engineering Team Level 1 Evaluation Criteria Assessment Form}

Alternative Number: 18 Alternative Title Direct Disposal to Grout

Date: 9/18/99

A. Evaluation Criterion Cost/Schedule

Title:

Evaluation Criterion Meet minimum combination of programmatic and technical risks and life cycle costs.

Description:

B. Evaluation Criterion ID \#: $\quad 6.0$ (Note 1)

C. Evaluation Criterion Weighted

$W_{1}=$ .12

Value:

D. Utility Functions:

Utility Function (UF) Value $\left(\mathrm{V}_{1}\right)=\Sigma$ Level 2 Criterion Weighted Score (WS)

(Note 2)

UF Value Formula:

(Note 3)

$$
\mathrm{V}_{1}=\mathrm{WS}_{6.1}+\mathrm{WS}_{6.2}+\mathrm{WS}_{6.3}
$$

F. Evaluation Criterion weighted score for the Alternative:

$\mathrm{W}_{1} \times \mathrm{V}_{1}=$ Weighted Score $\therefore \underline{.12} \times \underline{55.00}=$

Explanatory Notes for Weighted Score:

Notes:

1. For Level I Evaluation Criterion, the ID\# is described by X.O, where $X=1,2,3$, etc. For Level 2 Evaluation Criterion, the ID\# is described by X.I, X.2, X.3, etc. where ' $X$ ' is the Level 1 Evaluation Criterion ID\#.

2. Utility Function values range from 0 (least desirable) to 100 (most desirable).

3. If Level 2 Criterion are used, the sum of the Level 2 "Weighted Scores" must be multiplied by the Level 1 Weight to determine the Level 1 Weighted Score. 


\section{HLW Salt Disposition Systems Engineering Team Level 2 Evaluation Criteria Assessment Form}

Alternative Number: 18 Alternative Title Direct Disposal to Grout

Date: $9 / 18 / 99$

A. Evaluation Criterion Regulatory Schedule Commitments

Title:

Evaluation Criterion Description:

Maximize capability of disposing of radioactive wastes per Federal Facility Agreement (FFA) \& Site Treatment Plan (STP) schedules or earlier.

B. Evaluation Criterion ID \#: (Note 1)

C. Evaluation Criterion Weighted

$$
\mathbf{W}_{2}=
$$
.5 Value:

D. Utility Functions:

UF Value

(Note 2)

Utility Function (UF) Description:
100

$\underline{70}$

UF.3 Base STP or Federal Facility Agreement (FFA) requirements to close HLW tanks by committed dates $\underline{0}$ is not met.

E. UF VALUE:

$$
\mathbf{v}_{2}=
$$
10

Explanatory Notes for UF Selected: Licensing the SRS as a high level waste repository. Yucca Mountain has been unsuccessful in being licensed for 20 years.

F. Evaluation Criterion weighted score (WS) for the Alternative:

$$
\mathrm{W}_{2} \times \mathrm{V}_{2}=\mathrm{WS} \therefore \underline{.5} \times \underline{\mathbf{1 0}}=
$$
5.00

Notes:

1. For Level 1 Evaluation Criterion, the ID\# is described by X.O, where $X=1,2,3$,etc. For Level 2 Evaluation Criterion, the ID\# is described by X.1, X.2, X.3, etc. where ' $X$ ' is the Level 1 Evaluation Criterion ID\#.

2. Utility Function values range from 0 (least desirable) to 100 (most desirable).

3. If Level 2 Criterion are used, the sum of the Level 2 "Weighted Scores" must be multiplied by the Level 1 Weight to determine the Level I Weighted Score. 


\section{HLW Salt Disposition Systems Engineering Team \\ Level 2 Evaluation Criteria Assessment Form}

Alternative Number: 18 Alternative Title Direct Disposal to Grout

Date: 9/18/99

A. Evaluation Criterion

Life Cycle Costs (LCC)

Title:

Evaluation Criterion

Minimize LCC including TEC, OPC, and D\&D (excludes salvage and repository costs).

Description:

B. Evaluation Criterion ID \#: (Note 1)

C. Evaluation Criterion Weighted

$\mathbf{W}_{2}=$ .3 Value:

D. Utility Functions:

UF Value

(Note 2)

Utility Function (UF) Description:

$\underline{\mathrm{LCC}} \leq 2$ billion dollars.

$\underline{100}$

$\underline{L C C}$ is 4 billion dollars.

$\underline{50}$

UF. $3 \quad$ LCC is 8 billion dollars.

$\underline{25}$

UF.4 $\quad \underline{L C C} \geq 16$ billion dollars.

E.

UF VALUE:

$\mathbf{V}_{2}=$

100

Explanatory Notes for UF Selected: LCC equals 20 billion dollars. Based on the point estimate. WSRC-RP-98-00167

F. Evaluation Criterion weighted score (WS) for the Alternative:

$\mathrm{w}_{2} \times \mathrm{V}_{2}=\mathrm{WS} \therefore .3 \times 100=$

30.00

Notes:

1. For Level 1 Evaluation Criterion, the ID\# is described by X.0, where $X=1,2,3$,etc. For Level 2 Evaluation Criterion, the ID\# is described by X.1, X.2, X.3, etc. where ' $X$ ' is the Level I Evaluation Criterion ID\#.

2. Utility Function values range from 0 (least desirable) to 100 (most desirable).

3. If Level 2 Criterion are used, the sum of the Level 2 "Weighted Scores" must be multiplied by the Level 1 Weight to determine the Level 1 Weighted Score. 


\section{HLW Salt Disposition Systems Engineering Team} Level 2 Evaluation Criteria Assessment Form

Alternative Number: 18 Alternative Title Direct Disposal to Grout

Date: 9/18/99

A. Evaluation Criterion

Repository Costs

Title:

Evaluation Criterion Minimize cost for waste disposal off-site (Federal Repository).

Description:

B. Evaluation Criterion ID \#: (Note 1)

C. Evaluation Criterion Weighted

$\mathbf{W}_{2}=$ .2

Value:

6.3

D. Utility Functions:

UF Value

(Note 2)

Utility Function (UF) Description:

$\leqq 6000$ canisters for off-site disposal

$\underline{100}$

$\underline{68,000 \text { canisters for off-site disposal. }}$

$\underline{50}$

UF.3 $\geq 130,000$ canisters for off-site disposal

E.

UF VALUE:

$V_{2}=$ 100

Explanatory Notes for UF Selected: $\quad$ DWPF canister production remains at 6000.

F. Evaluation Criterion weighted score (WS) for the Alternative:

$\mathrm{W}_{2} \times \mathrm{V}_{2}=\mathrm{WS} \therefore .2 \times \underline{100}=\underline{20.00}$

Notes:

1. For Level 1 Evaluation Criterion, the ID\# is described by X.O, where $X=1,2,3$,etc. For Level 2 Evaluation Criterion, the ID\# is described by X.1, X.2, X.3, etc. where ' $X$ ' is the Level 1 Evaluation Criterion ID\#.

2. Utility Function values range from 0 (least desirable) to 100 (most desirable).

3. If Level 2 Criterion are used, the sum of the Level 2 "Weighted Scores" must be multiplied by the Level 1 Weight to determine the Level I Weighted Score. 
High Level Waste Salt Disposition

Systems Engineering Team

Final Report
WSRC-RP-99-00007

Revision: 0

Page 120 of 277

\subsubsection{Small Tank TPB Precipitation}




\section{HLW Salt Disposition Systems Engineering Team Level 1 Evaluation Criteria Assessment Form}

Alternative Number: 13 Alternative Title_ Small Tank TPB Precipitation

Date: $9 / 18 / 99$

A. Evaluation Criterion

Title: Technology

Evaluation Criterion Description: Maximize the confidence that underlying scientific principles \& engineering implementation will result in adequate attainment.

B. Evaluation Criterion ID \#: (Note 1)

C. Evaluation Criterion Weighted

$\mathbf{W}_{1}=$ .23

Value:

D. Utility Functions:

Utility Function (UF) Value $\left(V_{1}\right)=\Sigma$ Level 2 Criterion Weighted Score (WS)

(Note 2)

UF Value Formula: (Note 3)

$$
V_{1}=W_{1.1}+W S_{1.2}+W S_{1.3}
$$

F. Evaluation Criterion weighted score for the Alternative:

$$
\mathrm{W}_{1} \times \mathrm{V}_{1}=\text { Weighted Score } \therefore . .23 \times \underline{78.00}=
$$

Explanatory Notes for Weighted Score:

Notes:

1. For Level I Evaluation Criterion, the ID\# is described by X.0, where $X=1,2,3$, etc. For Level 2 Evaluation Criterion, the ID\# is described by X.I, X.2, X.3, etc. where ' $X$ ' is the Level I Evaluation Criterion ID\#.

2. Utility Function values range from 0 (least desirable) to 100 (most desirable).

3. If Level 2 Criterion are used, the sum of the Level 2 "Weighted Scores" must be multiplied by the Level 1 Weight to determine the Level 1 Weighted Score. 


\section{HLW Salt Disposition Systems Engineering Team Level 2 Evaluation Criteria Assessment Form}

Alternative Number: 13 Alternative Title___ Small Tank TPB Precipitation

Date: $9 / 18 / 99$

A. Evaluation Criterion Scientific Maturity

Title:

Evaluation Criterion

The level of scientific understanding needed to minimize project risk.

Description:

B. Evaluation Criterion ID \#:

(Note 1)

C. Evaluation Criterion Weighted

$\mathbf{W}_{2}=$ 4

Value:

D. Utility Functions:

UF Value

(Note 2)

Utility Function (UF) Description:

UF.1 Reliable radioactive production scale demonstration \& correlation to predicted scientific results.

$\underline{100}$

Large scale radioactive test: 'spiked' radiochemistry demonstration.

UF.3 Pilot (small) scale radioactive test; full radiochemistry.

UF.4 Lab scale test; simulant/real waste.

UF.5 Theoretical understanding only; no practical demonstration.

$\underline{0.0}$

E.

UF VALUE:

Explanatory Notes for UF Selected:
$\mathbf{V}_{2}=$ 80

ITP radioactive demonstration runs. DNFSB 96-1 SRTC laboratory work. 20L CSTR closed loop "spiked" radiochemistry demonstration. Precipitate foaming observed during radioactive CSTR bench scale test confirmed the need for anti-foam development. TPB recovery efficiency was $1 / 3$ of expectation.

F. Evaluation Criterion weighted score (WS) for the Alternative:

$$
W_{2} \times V_{2}=W S \therefore .4 \times .80=
$$

Notes:

1. For Level 1 Evaluation Criterion, the ID\# is described by X.O, where $X=1,2,3$, etc. For Level 2 Evaluation Criterion, the $I D \#$ is described by X.1, X.2, X.3, etc. where ' $X$ ' is the Level I Evaluation Criterion ID\#.

2. Utility Function values range from 0 (least desirable) to 100 (most desirable).

3. If Level 2 Criterion are used, the sum of the Level 2 "Weighted Scores" must be multiplied by the Level 1 Weight to determine the Level 1 Weighted Score. 


\section{HLW Salt Disposition Systems Engineering Team \\ Level 2 Evaluation Criteria Assessment Form}

Alternative Number: 13 Alternative Title__ Small Tank TPB Precipitation

Date:

$9 / 18 / 99$

A. Evaluation Criterion

Engineering Maturity

Title:

Evaluation Criterion

The level of applied engineering concepts needed to minimize project risk.

Description:

B. Evaluation Criterion $\mathrm{DD}$ \#:

(Note 1)

C. Evaluation Criterion Weighted

$\mathbf{W}_{2}=$ .4

Value:

D. Utility Functions:

UF Value

(Note 2)

Utility Function (UF) Description:

$\underline{100}$

$\underline{60}$

Reliable non-radioactive production scale with significant operating experience.

$\underline{40}$

UF.3 Limited radioactive production scale.

$\underline{20}$

UF.4 Limited non-radioactive production scale

UF.5 Demonstration

E.

UF VALUE:

$\mathbf{V}_{2}=$

80
ITP filter radioactive production scale operation. 20L CSTR closed loop "spiked" radiochemistry demonstration confirms continuous precipitation. Testing at various scales has indicated a need to address NaTPB dispersion.

F. Evaluation Criterion weighted score (WS) for the Alternative:

$\mathrm{W}_{2} \times \mathrm{V}_{2}=\mathrm{WS} \therefore \underline{.4} \times \underline{80}=$

32.00

Notes:

1. For Level 1 Evaluation Criterion, the ID\# is described by X.0, where $X=1,2,3$,etc. For Level 2 Evaluation Criterion, the $I D \#$ is described by X.1, X.2, X.3, etc. where ' $X$ ' is the Level 1 Evaluation Criterion ID\#.

2. Utility Function values range from 0 (least desirable) to 100 (most desirable).

3. If Level 2 Criterion are used, the sum of the Level 2 "Weighted Scores" must be multiplied by the Level 1 Weight to determine the Level I Weighted Score. 
HLW Salt Disposition Systems Engineering Team Level 2 Evaluation Criteria Assessment Form

Alternative Number: 13 Alternative Title__ Small Tank TPB Precipitation

Date: $9 / 18 / 99$

A. Evaluation Criterion

Title:

Evaluation Criterion Description:

Process Simplicity

Ease of Science implementation understanding by operators.

B. Evaluation Criterion ID \#: (Note 1)

C. Evaluation Criterion Weighted

$\mathbf{W}_{2}=$ .2 Value:

D. Utility Functions:

UF Value

(Note 2)

Utility Function (UF) Description:

Low complexity, straight forward operations.

$\underline{100}$

JF.2 Moderate complexity - operator aids and routine engineering support.

$\underline{70}$

UF.3 Complex - significant training for operators and continuous, specialized engineering support required.

E.

UF VALUE:

Explanatory Notes for UF Selected:

Similar operations to salt and chemical cells operations at DWPF, monitoring temperatures, flows, and product productions. WSRC-RP-99-00005; HLW-SDT-99-0266.

F. Evaluation Criterion weighted score (WS) for the Alternative:

Notes:

1. For Level 1 Evaluation Criterion, the ID\# is described by X.0, where $X=1,2,3$,etc. For Level 2 Evaluation Criterion, the $I D \#$ is described by X.I, X.2, X.3, etc. where ' $X$ ' is the Level 1 Evaluation Criterion ID\#.

2. Utility Function values range from 0 (least desirable) to 100 (most desirable).

3. If Level 2 Criterion are used, the sum of the Level 2 "Weighted Scores" must be multiplied by the Level 1 Weight to determine the Level I Weighted Score. 


\section{HLW Salt Disposition Systems Engineering Team Level 1 Evaluation Criteria Assessment Form}

Alternative Number: 13 Alternative Title_ Small Tank TPB Precipitation

Date: $9 / 18 / 99$

A. Evaluation Criterion Current Mission Interfaces

Title:

Evaluation Criterion Impact on current SRS missions/programs.

Description:

B. Evaluation Criterion ID \#: $\quad 2.0$ (Note 1)

C. Evaluation Criterion Weighted

$W_{1}=$ .15

Value:

D. Utility Functions:

Utility Function (UF) Value $\left(\mathrm{V}_{1}\right)=\Sigma$ Level 2 Criterion Weighted Score (WS)

(Note 2)

UF Value Formula: (Note 3)

$$
\mathrm{V}_{1}=\mathrm{WS}_{2.1}+\mathrm{WS}_{2.2}+\mathrm{WS}_{2.3}+\mathrm{WS}_{2.4}+\mathrm{WS}_{2.5}
$$

F. Evaluation Criterion weighted score for the Alternative:

$\mathbf{W}_{1} \times \mathrm{V}_{1}=$ Weighted Score

$.15 \times$

65.50 $=$

Explanatory Notes for Weighted Score:

Notes:

1. For Level 1 Evaluation Criterion, the ID\# is described by X.O, where $X=1,2,3$,etc. For Level 2 Evaluation Criterion, the ID\# is described by X.1, X.2, X.3, etc. where ' $X$ ' is the Level 1 Evaluation Criterion ID\#.

2. Utility Function values range from 0 (least desirable) to 100 (most desirable).

3. If Level 2 Criterion are used, the sum of the Level 2 "Weighted Scores" must be multiplied by the Level 1 Weight to determine the Level 1 Weighted Score. 


\section{HLW Salt Disposition Systems Engineering Team Level 2 Evaluation Criteria Assessment Form}

Alternative Number:_13 Alternative Title__ Small Tank TPB Precipitation

Date: $9 / 18 / 99$

A. Evaluation Criterion

DWPF

Title:

Evaluation Criterion Impact on DWPF (Table 1 Functions \& Requirements).

Description:

B. Evaluation Criterion ID \#: (Note 1)

C. Evaluation Criterion Weighted

$\mathbf{W}_{2}=$ .25 Value:

D. Utility Functions:

UF Value

(Note 2)

Utility Function (UF) Description:

\section{Sludge only to completion}

$\underline{100}$

Sludge plus MST to completion.

$\underline{85}$

$\underline{70}$

UF.3 Baseline - current ITP flowsheet.

$\underline{20}$

UF.4 Moderate impact - some additional canisters $(<50 \%)$. Facility modifications required.

$\underline{0}$

UF.5 Significant impact - additional canisters ( $>50 \%$ ) glass reformulation/repermitting required. Major facility modifications required.

E.

UF VALUE:

Explanatory Notes for UF Selected:
$\mathbf{V}_{2}=$ 85

Precipitate hydrolysis process removed from DWPF. Sludge, MST, and PHA streams. The product stream provides a soft interface with DWPF.

F. Evaluation Criterion weighted score (WS) for the Alternative:

$$
\mathrm{W}_{2} \times \mathrm{V}_{2}=\mathrm{WS} \therefore \underline{.25} \times \underline{85}=\underline{21.25}
$$

Notes:

1. For Level I Evaluation Criterion, the ID\# is described by X.O, where $X=1,2,3$,etc. For Level 2 Evaluation Criterion, the ID\# is described by X.1, X.2, X.3, etc. where ' $X$ ' is the Level 1 Evaluation Criterion ID\#.

2. Utility Function values range from 0 (least desirable) to 100 (most desirable).

3. If Level 2 Criterion are used, the sum of the Level 2 "Weighted Scores" must be multiplied by the Level 1 Weight to determine the Level 1 Weighted Score. 


\section{HLW Salt Disposition Systems Engineering Team} Level 2 Evaluation Criteria Assessment Form

Alternative Number: 13 Alternative Title__ Small Tank TPB Precipitation

Date: $9 / 18 / 99$

A. Evaluation Criterion

Saltstone

Title:

Evaluation Criterion Impact on Saltstone (Table 1 Functions \& Requirements).

Description:

B. Evaluation Criterion ID \#: (Note 1)

2.2

C. Evaluation Criterion Weighted

$\mathbf{W}_{2}=$ .15 Value:

D. Utility Functions:

UF Value

(Note 2)

Utility Function (UF) Description:

UF.1 No need for Saltstone Facility.

UF.3 180M gallons saltstone plus Benzene risk (current flowsheet).

UF.4 Moderate increase in saltstone $(<50 \%)$. Minor facility modifications.

UF.5 Repermit saltstone to Class C waste. Major facility modifications and increased throughtput $(>50 \%)$.

E.

UF VALUE:

$\mathbf{V}_{2}=$ 75

Explanatory Notes for UF Selected: $\quad$ Reduced benzene release.

F. Evaluation Criterion weighted score (WS) for the Alternative

$\mathrm{W}_{2} \times \mathrm{V}_{2}=\mathrm{WS} \therefore \underline{.15} \times \underline{75}=\underline{11.25}$

Notes:

1. For Level 1 Evaluation Criterion, the ID\# is described by X.0, where $X=1,2,3$,etc. For Level 2 Evaluation Criterion, the $I D \#$ is described by X.1, X.2, X.3, etc. where ' $X$ ' is the Level 1 Evaluation Criterion ID\#.

2. Utility Function values range from 0 (least desirable) to 100 (most desirable).

3. If Level 2 Criterion are used, the sum of the Level 2 "Weighted Scores" must be multiplied by the Level 1 Weight to determine the Level 1 Weighted Score. 


\section{HLW Salt Disposition Systems Engineering Team} Level 2 Evaluation Criteria Assessment Form

Alternative Number: 13

Alternative Title Small Tank TPB Precipitation

Date: 9/18/99

A. Evaluation Criterion

Title:

Evaluation Criterion Description:

Solid Waste

Impact on Solid Waste (Table 1 Functions \& Requirements).

B. Evaluation Criterion ID \#: (Note 1)

$\underline{2.3}$

C. Evaluation Criterion Weighted

$\mathrm{W}_{2}=$ .1 Value:

D. Utility Functions:

UF Value

(Note 2)

Utility Function (UF) Description:

UF.1 Reduced solid waste volume and no Benzene.

F.2 Reduced solid waste volume and Benzene.

UF.3 Current flowsheet (Benzene tocir)

UF.5 Repermit new waste forms, significant increase in solid waste volume.

E. UF VALUE:

$$
\mathbf{V}_{2}=
$$
50

Explanatory Notes for UF Selected:

Comparable to current flowsheet.

F. Evaluation Criterion weighted score (WS) for the Alternative:

$\mathrm{W}_{2} \times \mathrm{V}_{2}=\mathrm{WS} \therefore . .1 \times \underline{50}=$ 5.00

Notes:

1. For Level 1 Evaluation Criterion, the ID\# is described by X.0, where $X=1,2,3$,etc. For Level 2 Evaluation Criterion, the $I D \#$ is described by X.1, X.2, X.3, etc. where ' $X$ ' is the Level 1 Evaluation Criterion ID\#.

2. Utility Function values range from 0 (least desirable) to 100 (most desirable).

3. If Level 2 Criterion are used, the sum of the Level 2 "Weighted Scores" must be multiplied by the Level 1 Weight to determine the Level 1 Weighted Score. 


\section{HLW Salt Disposition Systems Engineering Team Level 2 Evaluation Criteria Assessment Form}

Alternative Number:_13 Alternative Title__ Small Tank TPB Precipitation

Date: 9/18/99

A. Evaluation Criterion Tank Farm

Title:

Evaluation Criterion Impact on Tank Farm (Table 1 Functions \& Requirements).

Description:

B. Evaluation Criterion ID \#: 2.4 (Note 1)

C. Evaluation Criterion Weighted

$\mathbf{W}_{2}=$ .2 Value:

D. Utility Functions:

Utility Function (UF) Description: impact. Current flowsheet.

UF.3 Increased safety hazards (e.g. Organics) increase operational capacity, increased corrosion impacts. $\underline{0}$

E.

UF VALUE:

$\mathbf{V}_{2}=$ 50

Explanatory Notes for UF Selected: Comparable to current flowsheet.

F. Evaluation Criterion weighted score (WS) for the Alternative:

$$
\mathrm{W}_{2} \times \mathrm{V}_{2}=\mathrm{WS} \therefore \underline{.2} \times \underline{50}=
$$

Notes:

1. For Level 1 Evaluation Criterion, the ID\# is described by X.O, where $X=1,2,3$,etc. For Level 2 Evaluation Criterion, the ID\# is described by X.1, X.2, X.3, etc. where ' $X$ ' is the Level 1 Evaluation Criterion ID\#.

2. Utility Function values range from 0 (least desirable) to 100 (most desirable).

3. If Level 2 Criterion are used, the sum of the Level 2 "Weighted Scores" must be multiplied by the Level 1 Weight to determine the Level 1 Weighted Score. 


\section{HLW Salt Disposition Systems Engineering Team} Level 2 Evaluation Criteria Assessment Form

Alternative Number: 13 Alternative Title__ Small Tank TPB Precipitation

Date: $9 / 18 / 99$

A. Evaluation Criterion Tank Farm Space Management

Title:

Evaluation Criterion Description:

Utilization of available Tank Farm storage \& resources as a function of time (HLW Salt Disposition Interface Functional Performance Requirement).

B. Evaluation Criterion ID \#: (Note 1)

$\underline{2.5}$

C. Evaluation Criterion Weighted

$\mathbf{W}_{2}=$ .3 Value:

D. Utility Functions:

UF Value

(Note 2)

$\underline{100}$

$\underline{40}$

UF.2 Current flowsheet (reduces available tank space)

$\underline{0}$
E.

UF VALUE:

Explanatory Notes for UF Selected:
$\mathbf{V}_{2}=$ 60

TK49 readily available for waste storage. TK48 available after waste handling strategy completed (could be processed by this flowsheet). WSRC-RP-99-0005; WSRC-RP-99$\underline{0006}$

F. Evaluation Criterion weighted score (WS) for the Alternative:

$\mathrm{W}_{2} \times \mathrm{V}_{2}=\mathrm{WS} \therefore \underline{.3} \times \underline{60}=$ 18.00

Notes:

1. For Level 1 Evaluation Criterion, the ID\# is described by X.O, where $X=1,2,3$,etc. For Level 2 Evaluation Criterion, the $I D \#$ is described by X.I, X.2, X.3, etc. where ' $X$ ' is the Level I Evaluation Criterion ID\#.

2. Utility Function values range from 0 (least desirable) to 100 (most desirable).

3. If Level 2 Criterion are used, the sum of the Level 2 "Weighted Scores" must be multiplied by the Level 1 Weight to determine the Level 1 Weighted Score. 


\section{HLW Salt Disposition Systems Engineering Team Level 1 Evaluation Criteria Assessment Form}

Alternative Number: 13 Alternative Title__ Small Tank TPB Precipitation

Date: $9 / 18 / 99$

A. Evaluation Criterion Future Mission Interfaces

Title:

Evaluation Criterion Maximize the support of identified potential future missions

Description:

B. Evaluation Criterion ID \#: (Note 1)

3.0

C. Evaluation Criterion Weighted

$\mathbf{W}_{1}=$ .07

Value:

D. Utility Functions:

UF Value

(Note 2)

Utility Function (UF) Description:

UF.1 Flexible system capable of supporting identified potential future missions. $\quad 100$

UF.2 System will support can-in-can and spent fuel stabilization. $\quad$ 70

UF.3 System will not support can-in-can or spent fuel stabilization. $\quad \underline{0}$

E.

UF VALUE:

$\mathrm{V}_{1}=$ 70

Explanatory Notes for UF Selected:

Cesium loading supports can-in-can mission. Tank space gain supports spent fuel stabilization mission.

F. Evaluation Criterion weighted score (WS) for the Alternative:

$\mathrm{W}_{1} \times \mathrm{V}_{1}=\mathrm{WS} \therefore \underline{.07} \times \underline{\mathbf{7 0}}=$

4.90

Notes:

1. For Level 1 Evaluation Criterion, the ID\# is described by X.O, where $X=1,2,3$,etc. For Level 2 Evaluation Criterion, the ID\# is described by X.I, X.2, X.3, etc. where ' $X$ ' is the Level 1 Evaluation Criterion ID\#.

2. Utility Function values range from 0 (least desirable) to 100 (most desirable).

3. If Level 2 Criterion are used, the sum of the Level 2 "Weighted Scores" must be multiplied by the Level 1 Weight to determine the Level 1 Weighted Score. 


\section{HLW Salt Disposition Systems Engineering Team} Level 1 Evaluation Criteria Assessment Form

Alternative Number: 13 Alternative Title Small Tank TPB Precipitation

Date: 9/18/99

A. Evaluation Criterion

Title:

Evaluation Criterion Description:

\section{Regulatory/ISMS/Environmental}

Protect personnel \& the environment from hazards \& releases of waste \& pollution by ensuring maximum application of intrinsic safety features.

B. Evaluation Criterion ID \#: (Note 1)

C. Evaluation Criterion Weighted

$\mathrm{w}_{1}=$ .23 Value:

D. Utility Functions:

Utility Function (UF) Value $\left(\mathrm{V}_{1}\right)=\Sigma$ Level 2 Criterion Weighted Score (WS)

(Note 2)

E. UF Value Formula: (Note 3)

$$
\mathrm{V}_{1}=\underline{W S}_{4.1}+\mathrm{WS}_{4.2}+\mathrm{WS}_{4.3}
$$

F. Evaluation Criterion weighted score for the Alternative: $\quad W_{1} \times V_{1}=$ Weighted Score $\therefore . .23 \times \underline{66.00}=$ Explanatory Notes for Weighted Score:

Notes:

1. For Level I Evaluation Criterion, the ID\# is described by X.0, where $X=1,2,3$,etc. For Level 2 Evaluation Criterion, the ID\# is described by X.I, X.2, X.3, etc. where ' $X$ ' is the Level 1 Evaluation Criterion ID\#.

2. Utility Function values range from 0 (least desirable) to 100 (most desirable).

3. If Level 2 Criterion are used, the sum of the Level 2 "Weighted Scores" must be multiplied by the Level 1 Weight to determine the Level 1 Weighted Score. 


\section{HLW Salt Disposition Systems Engineering Team Level 2 Evaluation Criteria Assessment Form}

Alternative Number: 13 Alternative Title_ Small Tank TPB Precipitation

Date: $9 / 18 / 99$

A. Evaluation Criterion Title:

Evaluation Criterion Description: Public/Environment

Protect the public \& environment from hazards \& accidental releases of waste \& pollution by ensuring maximum application of intrinsic safety features.

B. Evaluation Criterion ID \#: (Note 1)

C. Evaluation Criterion Weighted

$\mathbf{W}_{2}=$ .45 Value:

D. Utility Functions:

UF Value

(Note 2)

Utility Function (UF) Description:

Process is inherently safe and can be quantified/documented in Authorization Basis.

$\underline{100}$

UF.2 Process has moderate hazards that are passively mitigated.

$\underline{85}$

UF.3 Process has moderate hazards that are readily mitigated.

$\underline{60}$

UF.4 Process has inherent hazards that can be mitigated with Engineered Safety Features and Administrative Controls.

UF.5 Process has inherent hazards and the risks are not quantifiable.

E.

UF VALUE:

$\mathbf{v}_{2}=$ 60

Explanatory Notes for UF Selected: Lower source term and lower energy source.

F. Evaluation Criterion weighted score (WS) for the Alternative:

$W_{2} \times V_{2}=W S \therefore \underline{.45} \times \underline{60}=$ 27.00

Notes:

1. For Level 1 Evaluation Criterion, the ID\# is described by X.O, where $X=1,2,3$,etc. For Level 2 Evaluation Criterion, the $I D \#$ is described by X.1, X.2, X.3, etc. where ' $X$ ' is the Level 1 Evaluation Criterion ID\#.

2. Utility Function values range from 0 (least desirable) to 100 (most desirable).

3. If Level 2 Criterion are used, the sum of the Level 2 "Weighted Scores" must be multiplied by the Level 1 Weight to determine the Level 1 Weighted Score. 


\section{HLW Salt Disposition Systems Engineering Team Level 2 Evaluation Criteria Assessment Form}

Alternative Number: 13 Alternative Title__ Small Tank TPB Precipitation

Date: $9 / 18 / 99$

A. Evaluation Criterion

Worker

Title:

Evaluation Criterion Description:

Protect on-site personnel from hazards \& accidental releases of waste \& pollution by ensuring maximum application of intrinsic safety features.

B. Evaluation Criterion ID \#:

(Note 1)

4.2

C. Evaluation Criterion Weighted

$\mathbf{W}_{2}=$ .35

Value:

D. Utility Functions:

UF Value

(Note 2)

Utility Function (UF) Description:

UF.1 Process is inherently safe and poses no unusual worker safety hazard.

$\underline{100}$

Process has moderate hazards that are passively mitigated.

$\underline{80}$

$\underline{60}$

UF.3 Process has moderate hazards that are readily mitigated.

$\underline{40}$

UF.4 Process has inherent hazards that can be mitigated with Structures, Systems, Components and Administrative Controls.

UF.5 Process has inherent hazards and poses significant risk to worker safety that are not readily mitigated. $\underline{0}$

E.

UF VALUE:

$\mathbf{V}_{2}=$ 60

Explanatory Notes for UF Selected: $\quad$ Reduced benzene releases. Precipitate hydrolysis process operation.

F. Evaluation Criterion weighted score (WS) for the Alternative:

$$
\mathrm{W}_{2} \times \mathrm{V}_{2}=\mathrm{WS} \therefore \underline{.35} \times \underline{60}=\underline{21.00}
$$

Notes:

1. For Level I Evaluation Criterion, the ID\# is described by X.0, where $X=1,2,3$,etc. For Level 2 Evaluation Criterion, the ID\# is described by $X .1, X .2, X .3$, etc. where ' $X$ ' is the Level 1 Evaluation Criterion ID\#.

2. Utility Function values range from 0 (least desirable) to 100 (most desirable).

3. If Level 2 Criterion are used, the sum of the Level 2 "Weighted Scores" must be multiplied by the Level 1 Weight to determine the Level 1 Weighted Score. 


\section{HLW Salt Disposition Systems Engineering Team} Level 2 Evaluation Criteria Assessment Form

Alternative Number: $13 \quad$ Alternative Title__ Small Tank TPB Precipitation

Date: $9 / 18 / 99$

A. Evaluation Criterion Permitting

Title:

Evaluation Criterion

Minimize waste generation risk \& difficulty of permitting new releases \& waste forms.

Description:

B. Evaluation Criterion ID \#: (Note 1)

C. Evaluation Criterion Weighted

$\mathbf{W}_{2}=$ .2 Value:

4.3

D. Utility Functions:

UF Value

Utility Function (UF) Description:

(Note 2)

UF.1 No new waste forms requiring permitting, eliminate one or more existing releases, no requalification $\quad 100$ of existing waste forms.

Reduction in current releases, no additional permitting required.

$\underline{80}$

UF.3 Current flowsheet (Saltstone Facility needs repermitting due to Benzene releases). $\quad \underline{60}$

UF.4 Requalification of existing waste form, exceeds current release levels. $\quad \underline{20}$

UF.5 New waste form permit required, significant increase in environmental releases requiring $\quad \underline{0}$ repermitting, high level waste retained in South Carolina

E. UF VALUE:

Explanatory Notes for UF Selected:

$$
\mathbf{V}_{2}=
$$
90 $\underline{6}$

$\underline{0}$ 


\section{HLW Salt Disposition Systems Engineering Team Level 1 Evaluation Criteria Assessment Form}

Alternative Number: 13 Alternative Title__ Small Tank TPB Precipitation

Date: $9 / 18 / 99$

A. Evaluation Criterion

Title:

Evaluation Criterion

Description:

Engineering (Design)

Maximize the confidence that the facility meets applicable codes, standards \& required production throughput.

B. Evaluation Criterion ID \#:

(Note 1)

C. Evaluation Criterion Weighted

$W_{1}=$ .2

Value:

5.0

D. Utility Functions:

Utility Function (UF) Value $\left(\mathrm{V}_{1}\right)=\Sigma$ Level 2 Criterion Weighted Score (WS)

(Note 2)

UF Value Formula: $\quad \mathbf{V}_{1}=\mathbf{W S}_{5.1}+\mathrm{WS}_{\underline{5.2}}+\mathbf{W S}_{\underline{5.3}}+\mathbf{W S}_{5.4}$

(Note 3)

F. Evaluation Criterion weighted score for the Alternative: $\quad W_{1} \times V_{1}=$ Weighted Score $\therefore . .2 \times \underline{70.00}=$

Explanatory Notes for Weighted Score:

Notes:

1. For Level 1 Evaluation Criterion, the ID\# is described by X.0, where $X=1,2,3$,etc. For Level 2 Evaluation Criterion, the ID\# is described by X.1, X.2, X.3, etc. where ' $X$ ' is the Level 1 Evaluation Criterion ID\#.

2. Utility Function values range from 0 (least desirable) to 100 (most desirable).

3. If Level 2 Criterion are used, the sum of the Level 2 "Weighted Scores" must be multiplied by the Level I Weight to determine the Level 1 Weighted Score. 


\section{HLW Salt Disposition Systems Engineering Team} Level 2 Evaluation Criteria Assessment Form

Alternative Number: 13 Alternative Title_ Small Tank TPB Precipitation

Date: $9 / 18 / 99$

A. Evaluation Criterion Construct

Title:

Evaluation Criterion Description:

Ensure facility design considers major construction/testing methods and needs in accordance with Integrated Work Process (IWP) and Key Activities for Successful Execution (KASE).

B. Evaluation Criterion ID \#:

(Note 1)

C. Evaluation Criterion Weighted

$\mathbf{W}_{\mathbf{2}}=$ .25

Value:

D. Utility Functions:

UF Value

(Note 2)

Utility Function (UF) Description:

$\underline{100}$

Facility design features and construction methods lead to simplicity of construction/testing process.

$\underline{60}$

complexity).

UF.3 Facility design features and construction methods are difficult to apply due to non-standard, noncommercial methods not readily applied in radioactive environment.

E.

UF VALUE:

$\mathbf{V}_{2}=$ 80

Explanatory Notes for UF Selected: Multiple unit operations coupled together.

F. Evaluation Criterion weighted score (WS) for the Alternative:

$\mathrm{W}_{2} \times \mathrm{V}_{2}=\mathrm{WS} \therefore .25 \times \underline{80}=\underline{20.00}$

Notes:

1. For Level 1 Evaluation Criterion, the ID\# is described by X.0, where $X=1,2,3$,etc. For Level 2 Evaluation Criterion, the ID\# is described by X.1, X.2, X.3, etc. where ' $X$ ' is the Level I Evaluation Criterion ID\#.

2. Utility Function values range from 0 (least desirable) to 100 (most desirable).

3. If Level 2 Criterion are used, the sum of the Level 2 "Weighted Scores" must be multiplied by the Level 1 Weight to determine the Level 1 Weighted Score. 


\section{HLW Salt Disposition Systems Engineering Team \\ Level 2 Evaluation Criteria Assessment Form}

Alternative Number:_13 Alternative Title__ Small Tank TPB Precipitation

Date: 9/18/99

A. Evaluation Criterion

Title:

Evaluation Criterion

Description:

Qualify

Readily validate defined functional design requirements, regulatory requirements, final disposal forms, and Authorization Basis ( $\mathrm{AB}$ ) safety requirements.

B. Evaluation Criterion ID \#:

(Note 1)

$\underline{5.2}$

C. Evaluation Criterion Weighted

$\mathbf{W}_{2}=$ .25

Value:

D. Utility Functions:

UF Value

(Note 2)

Utility Function (UF) Description:

Test program

Test program applies "Graded Approach" to verify key design attributes with other limited testing"

$\underline{70}$ and inferred results.

UF.3 Insufficient science/engineering exists to establish firm test acceptance criteria and methods, limited $\underline{0}$ direct verification.

E.

UF VALUE:

Explanatory Notes for UF Selected: $\mathbf{v}_{2}=$ 80

Majority of design attributes provide for direct verification. Process variables will require some inferred results.

F. Evaluation Criterion weighted score (WS) for the Alternative

$\mathrm{W}_{2} \times \mathrm{V}_{2}=\mathrm{WS} \therefore .25 \times \underline{80}=\underline{20.00}$

Notes:

1. For Level 1 Evaluation Criterion, the ID\# is described by X.O, where $X=1,2,3$,etc. For Level 2 Evaluation Criterion, the $I D \#$ is described by X.1, X.2, X.3, etc. where ' $X$ ' is the Level 1 Evaluation Criterion ID\#.

2. Utility Function values range from 0 (least desirable) to 100 (most desirable).

3. If Level 2 Criterion are used, the sum of the Level 2 "Weighted Scores" must be multiplied by the Level 1 Weight to determine the Level 1 Weighted Score. 


\section{HLW Salt Disposition Systems Engineering Team Level 2 Evaluation Criteria Assessment Form}

Alternative Number: $13 \quad$ Alternative Title__ Small Tank TPB Precipitation

Date: $9 / 18 / 99$

A. Evaluation Criterion

Operate

Title:

Evaluation Criterion

Description:

Maximize ease of repeat operation/proceduralization, access for round sheets/physical verification, and upset operation management (Section R-1.4-3 of Functions \& Requirements).

B. Evaluation Criterion ID \#: $\quad 5.3$ (Note 1)

C. Evaluation Criterion Weighted

$\mathbf{W}_{2}=$ .25 Value:

D. Utility Functions:

UF Value

Utility Function (UF) Description:

(Note 2)

Design allows simple, coordinated, straight forward operation with direct access to key controls, interlocks, and instruments. Easy access to key equipment, maximize ALARA considerations. Minimize number of process control points.

UF.2 Design allows manageable operation with minimal complexity (Standard SRS practice).

UF.3 Design is highly coupled with minimum holdup, multiple parallel operations and fast dynamics, and $\underline{0}$ process instability.

E. UF VALUE:

$\mathbf{V}_{2}=$ 60

Explanatory Notes for UF Selected: $\quad$ Single facility for salt solution processing comparable to standard SRS practices.

F. Evaluation Criterion weighted score (WS) for the Alternative:

$\mathrm{W}_{2} \times \mathrm{V}_{2}=\mathrm{WS} \therefore \underline{.25} \times \underline{60}=\underline{15.00}$

Notes:

1. For Level I Evaluation Criterion, the ID\# is described by X.O, where $X=1,2,3$,etc. For Level 2 Evaluation Criterion, the ID\# is described by X.1, X.2, X.3, etc. where ' $X$ ' is the Level 1 Evaluation Criterion ID\#.

2. Utility Function values range from 0 (least desirable) to 100 (most desirable).

3. If Level 2 Criterion are used, the sum of the Level 2 "Weighted Scores" must be multiplied by the Level 1 Weight to determine the Level 1 Weighted Score. 


\section{HLW Salt Disposition Systems Engineering Team Level 2 Evaluation Criteria Assessment Form}

Alternative Number: 13 Alternative Title__ Small Tank TPB Precipitation

Date: $9 / 18 / 99$

A. Evaluation Criterion

RAMI

Title:

Evaluation Criterion

Design to maximize Reliability, Availability, Maintainability, and Inspectability.

Description:

B. Evaluation Criterion ID \#:

(Note 1)

C. Evaluation Criterion Weighted

$\mathbf{W}_{2}=$ .25

Value:

5.4

D. Utility Functions:

UF Value

(Note 2)

Utility Function (UF) Description:

JF.1 Design allows simple, coordinated, straight forward maintenance practices which take into account

ALARA requirements. Design maximizes reliability, and availability of Structures. Systems, and

Components.

UF.2 Design allows manageable maintenance functions with minimal complexibility (Standard SRS practice).

UF.3 Design complexity restricts maintainability and inspectability and reduces reliability, availability of Structures, Systems, and Components. Remoteability restricts maintainability.

E.

UF VALUE:

$\mathbf{V}_{2}=$ 60

Explanatory Notes for UF Selected: $\quad$ Single facility for salt solution processing comparable to standard SRS practice.

F. Evaluation Criterion weighted score (WS) for the Alternative:

$\mathrm{W}_{2} \times \mathrm{V}_{2}=\mathrm{WS} \therefore .25 \times \underline{60}=\underline{15.00}$

Notes:

1. For Level 1 Evaluation Criterion, the ID\# is described by X.O, where $X=1,2,3$,etc. For Level 2 Evaluation Criterion, the ID\# is described by X.I, X.2, X.3, etc. where ' $X$ ' is the Level 1 Evaluation Criterion ID\#.

2. Utility Function values range from 0 (least desirable) to 100 (most desirable).

3. If Level 2 Criterion are used, the sum of the Level 2 "Weighted Scores" must be multiplied by the Level 1 Weight to determine the Level 1 Weighted Score. 


\section{HLW Salt Disposition Systems Engineering Team} Level 1 Evaluation Criteria Assessment Form

Alternative Number: Alternative Title Small Tank TPB Precipitation

Date: $9 / 18 / 99$

A. Evaluation Criterion Cost/Schedule Title:

Evaluation Criterion Description:

B. Evaluation Criterion ID \#: (Note 1)

$\underline{6.0}$

C. Evaluation Criterion Weighted

$\mathrm{W}_{1}=$ .12

Value:

D. Utility Functions:

Utility Function (UF) Value $\left(\mathrm{V}_{1}\right)=\Sigma$ Level 2 Criterion Weighted Score (WS)

(Note 2)

UF Value Formula: (Note 3)

$$
\mathbf{V}_{1}=\underline{W_{6.1}}+\mathbf{W S}_{\underline{6.2}}+\mathbf{W S}_{\underline{6.3}}
$$
(Note 3)

F. Evaluation Criterion weighted score for the Alternative: $\mathrm{W}_{1} \times \mathrm{V}_{1}=$ Weighted Score $\therefore$ $\therefore .12$ 78.00 Explanatory Notes for Weighted Score:

Notes:

1. For Level 1 Evaluation Criterion, the ID\# is described by X.0, where $X=1,2,3$, etc. For Level 2 Evaluation Criterion, the ID\# is described by X.1, X.2, X.3, etc. where ' $X$ ' is the Level 1 Evaluation Criterion ID\#.

2. Utility Function values range from 0 (least desirable) to 100 (most desirable).

3. If Level 2 Criterion are used, the sum of the Level 2 "Weighted Scores" must be multiplied by the Level I Weight to determine the Level 1 Weighted Score. 


\section{HLW Salt Disposition Systems Engineering Team Level 2 Evaluation Criteria Assessment Form}

Alternative Number:_13 Alternative Title__ Small Tank TPB Precipitation

Date: 9/18/99

A. Evaluation Criterion Regulatory Schedule Commitments

Title:

Evaluation Criterion Description:

B. Evaluation Criterion ID \#: (Note 1)

C. Evaluation Criterion Weighted

$\mathbf{W}_{2}=$ .5 Value:

D. Utility Functions:

UF Value

(Note 2)

Utility Function (UF) Description:

UF.1 Accelerated Cleanup Plan (ACP) to empty High Level Waste (HLW) tanks by 2022 is met.

UF.2 Base Site Treatment Plan (STP) requirement to close HLW tanks by 2028 is met.

$\underline{70}$

UF.3 Base STP or Federal Facility Agreement (FFA) requirements to close HLW tanks by committed dates $\underline{0}$ is not met.

E.

UF VALUE:

Explanatory Notes for UF Selected:

DWPF operation supports STP requirements. Flexibility to expand throughput, but would not meet ACP.

F. Evaluation Criterion weighted score (WS) for the Alternative:

$\mathbf{W}_{2} \times \mathrm{V}_{2}=\mathrm{WS} \therefore \underline{.5} \times \underline{\mathbf{8 0}}=$ 40.00

Notes:

1. For Level 1 Evaluation Criterion, the ID\# is described by X.0, where $X=1,2,3$,etc. For Level 2 Evaluation Criterion, the $I D \#$ is described by X.1, X.2, X.3, etc. where ' $X$ ' is the Level 1 Evaluation Criterion ID\#.

2. Utility Function values range from 0 (least desirable) to 100 (most desirable).

3. If Level 2 Criterion are used, the sum of the Level 2 "Weighted Scores" must be multiplied by the Level 1 Weight to determine the Level 1 Weighted Score. 


\section{HLW Salt Disposition Systems Engineering Team Level 2 Evaluation Criteria Assessment Form}

Alternative Number: 13 Alternative Title__ Small Tank TPB Precipitation

Date: $9 / 18 / 99$

A. Evaluation Criterion

Title:

Evaluation Criterion Life Cycle Costs (LCC)

Description:

Minimize LCC including TEC, OPC, and D\&D (excludes salvage and repository costs)

B. Evaluation Criterion ID \#: (Note 1)

C. Evaluation Criterion Weighted

$\mathbf{W}_{2}=$ .3 Value:

6.2

D. Utility Functions:

Utility Function (UF) Description:

UF Value

(Note 2)

$\underline{\mathrm{LCC}} \leq 2$ billion dollars.

$\underline{100}$

$\underline{\mathrm{LCC}}$ is 4 billion dollars.

UF.3 LCC is 8 billion dollars.

$\underline{0}$

UF.4 $\quad \underline{L C C} \geq 16$ billion dollars.

E.

UF VALUE:

$$
\mathbf{V}_{2}=
$$
60

Explanatory Notes for UF Selected: $\quad$ LCC equals 3.5 billion dollars. Based on past estimate. WSRC-RP-98-00167

F. Evaluation Criterion weighted score (WS) for the Alternative:

$\mathrm{W}_{2} \times \mathrm{V}_{2}=\mathrm{WS} \therefore \underline{.3} \times \underline{60}=$ 18.00

Notes:

1. For Level I Evaluation Criterion, the ID\# is described by X.0, where $X=1,2,3$,etc. For Level 2 Evaluation Criterion, the $I D \#$ is described by X.1,X.2, X.3, etc. where ' $X$ ' is the Level 1 Evaluation Criterion ID\#.

2. Utility Function values range from 0 (least desirable) to 100 (most desirable).

3. If Level 2 Criterion are used, the sum of the Level 2 "Weighted Scores" must be multiplied by the Level 1 Weight to determine the Level I Weighted Score. 


\section{HLW Salt Disposition Systems Engineering Team Level 2 Evaluation Criteria Assessment Form}

Alternative Number: 13 Alternative Title__ Small Tank TPB Precipitation

Date: $9 / 18 / 99$

A. Evaluation Criterion

Title:

Evaluation Criterion

Description:

Repository Costs

Minimize cost for waste disposal off-site (Federal Repository).

B. Evaluation Criterion ID \#: $\quad 6.3$

(Note 1)

C. Evaluation Criterion Weighted

$\mathbf{W}_{2}=$ .2

Value:

D. Utility Functions:

UF Value

(Note 2)

Utility Function (UF) Description:

$\leqq 6000$ canisters for off-site disposal

$\underline{100}$

$\underline{68,000 \text { canisters for off-site disposal. }}$

$\underline{50}$

UF.3 $\geqq 130,000$ canisters for off-site disposal

E.

UF VALUE:

Explanatory Notes for UF Selected:

DWPF canister production remains at 6000

F. Evaluation Criterion weighted score (WS) for the Alternative:

$\mathrm{W}_{2} \times \mathrm{V}_{2}=\mathrm{WS} \therefore \underline{.2} \times \underline{100}=\underline{20.00}$

Notes:

1. For Level I Evaluation Criterion, the ID\# is described by X.O, where $X=1,2,3$, etc. For Level 2 Evaluation Criterion, the 'ID\# is described by X.1, X.2, X.3, etc. where ' $X$ ' is the Level 1 Evaluation Criterion ID\#.

2. Utility Function values range from 0 (least desirablepto 100 (most desirable).

3. If Level 2 Criterion are used, the sum of the Level 2 "Weighted Scores" must be multiplied by the Level I Weight to determine the Level 1 Weighted Score. 


\subsection{Cost Validation Matrix}

\subsubsection{Caustic Side Solvent Extraction}

\begin{tabular}{|c|c|c|c|c|c|c|c|c|c|c|c|c|c|}
\hline \multirow{2}{*}{$\begin{array}{l}\text { ID } \\
\text { No }\end{array}$} & \multirow{2}{*}{$\begin{array}{c}\text { Caustic Side } \\
\text { Solvent Extraction } \\
\text { Uncertainty } \\
\text { Statement }\end{array}$} & \multicolumn{9}{|c|}{$\begin{array}{c}\text { Areas of } \\
\text { Uncertainty }\end{array}$} & \multirow[b]{2}{*}{1} & \multirow[b]{2}{*}{2} & \multirow[b]{2}{*}{ Explanatory Notes } \\
\hline & & Mission & $\begin{array}{l}\text { Technical } \\
\text { Maturity }\end{array}$ & Environmental & $\begin{array}{c}\text { Engineering/ } \\
\text { Designg }\end{array}$ & Operation & Regulatory & Stakeholder & Safety & $\begin{array}{l}\text { Radiological } \\
\end{array}$ & & & \\
\hline 1 & $\begin{array}{l}\text { Decomposition/De } \\
\text { gradation products } \\
\text { may negatively } \\
\text { affect downstream } \\
\text { operations. }\end{array}$ & & $\bar{x}$ & & & & & & & & $\mathrm{X}$ & & $\begin{array}{l}\$ 1 \text { million cost } \\
\text { increase for } 2 \\
\text { carbon bed filters. } \\
\text { No change. }\end{array}$ \\
\hline 2 & $\begin{array}{l}\text { MST amount } \\
\text { needed for } \\
\text { decontamination } \\
\text { exceeds DWPF Ti } \\
\text { glass limits. }\end{array}$ & & & & & & $\mathrm{x}$ & & & & & $X$ & $\begin{array}{l}\text { MST concentration } \\
\text { of } 0.4 \mathrm{~g} / \mathrm{L} \text { makes } \\
\text { acceptable glass. } \\
\text { WSRC-TR-99- } \\
00332\end{array}$ \\
\hline 3 & $\begin{array}{l}\text { Crud formation in } \\
\text { the system at the } \\
\text { organic to aqueous } \\
\text { interface. }\end{array}$ & & $\mathrm{x}$ & & & & & & & & $\mathrm{X}$ & & $\begin{array}{l}\$ 500,000 \text { cost } \\
\text { increase for crud } \\
\text { separation tanks. } \\
\text { No change. }\end{array}$ \\
\hline
\end{tabular}




\begin{tabular}{|c|c|c|c|c|c|c|c|c|c|c|c|c|c|}
\hline \multirow{2}{*}{$\begin{array}{l}\text { ID } \\
\text { No }\end{array}$} & \multirow{2}{*}{$\begin{array}{c}\text { Caustic Side } \\
\text { Solvent Extraction } \\
\text { Uncertainty } \\
\text { Statement } \\
\end{array}$} & \multicolumn{9}{|c|}{$\begin{array}{c}\text { Areas of } \\
\text { Uncertainty }\end{array}$} & \multirow[b]{2}{*}{1} & \multirow[b]{2}{*}{2} & \multirow[b]{2}{*}{ Explanatory Notes } \\
\hline & & Mission & $\begin{array}{l}\text { Technical } \\
\text { Maturity }\end{array}$ & Environmental & $\begin{array}{c}\text { Engineering / } \\
\text { Design }\end{array}$ & Operation & Regulatory & $\begin{array}{l}\text { Stakeholder } \\
\end{array}$ & Safety & Radiological & & & \\
\hline 4 & $\begin{array}{l}\text { Insufficient } \\
\text { understanding of } \\
\text { the operating } \\
\text { window with } \\
\text { respect to feed } \\
\text { impurities. } \\
\text { (DNFSB 96-1) }\end{array}$ & & $\bar{x}$ & & & & & & & & $\bar{X}$ & & $\begin{array}{l}14 \text { month delay in } \\
\text { completing } \\
\text { preliminary design. } \\
\text { Some anionic } \\
\text { impurities work } \\
\text { was completed. } \\
\text { HLW-SDT-99- } \\
0283 \\
\text { No change. }\end{array}$ \\
\hline 5 & $\begin{array}{l}\text { Difficulty in } \\
\text { filtration of sludge } \\
\text { and/or MST will } \\
\text { produce low filtrate } \\
\text { flow rates and } \\
\text { require frequent } \\
\text { cleaning }\end{array}$ & & & & $\bar{x}$ & & & & & & $\mathrm{X}$ & & $\begin{array}{l}\$ 6 \text { million cost } \\
\text { increase for the } \\
\text { larger filters. } \\
\$ 10 \text { million cost } \\
\text { increase for the } \\
\text { larger pumps. Flux } \\
\text { rate decrease from } \\
0.07 \text { to } 0.02 \text {. } \\
\text { WSRC-TR-99- } \\
00342\end{array}$ \\
\hline
\end{tabular}




\begin{tabular}{|c|c|c|c|c|c|c|c|c|c|c|c|c|c|}
\hline \multirow{2}{*}{$\begin{array}{l}\text { ID } \\
\text { No }\end{array}$} & \multirow{2}{*}{$\begin{array}{c}\text { Caustic Side } \\
\text { Solvent Extraction } \\
\text { Uncertainty } \\
\text { Statement }\end{array}$} & \multicolumn{9}{|c|}{$\begin{array}{c}\text { Areas of } \\
\text { Uncertainty }\end{array}$} & \multirow[b]{2}{*}{1} & \multirow[b]{2}{*}{2} & \multirow[b]{2}{*}{ Explanatory Notes } \\
\hline & & Mission & $\begin{array}{l}\text { Technical } \\
\text { Maturity }\end{array}$ & Environmental & $\begin{array}{c}\begin{array}{c}\text { Engineering } / \\
\text { Design }\end{array} \\
\end{array}$ & Operation & Regulatory & Stakeholder & Safety & Radiological & & & \\
\hline 6 & $\begin{array}{l}\text { TRU } \\
\text { decontamination } \\
\text { with MST is not } \\
\text { adequate with the } \\
\text { design residence } \\
\text { time. }\end{array}$ & & $\overline{\mathrm{X}}$ & & & & & & & & $\mathrm{X}$ & & $\begin{array}{l}125 \mathrm{Kgal} \text {. double } \\
\text { lobe tank for } \\
\text { dilution effect. } \\
\text { MST concentration } \\
\text { and residence time } \\
\text { requires more } \\
\text { research for } \\
\text { bounding waste. } \\
\text { No additional cost } \\
\text { beyond Item } 27 \text {. }\end{array}$ \\
\hline 7 & $\begin{array}{l}\text { Analysis delay (1 } \\
\text { week) in measuring } \\
\text { for Sr DF in MST } \\
\text { process. }\end{array}$ & & $\bar{x}$ & & & & & & & & $\bar{X}$ & & $\begin{array}{l}\text { No cost or schedule } \\
\text { impact, within } \\
\text { existing R\&D } \\
\text { scope and schedule } \\
\text { duration. } \\
\text { No change. }\end{array}$ \\
\hline 8 & $\begin{array}{l}\text { What is the fate of } \\
\text { Am in the process? }\end{array}$ & & $\bar{x}$ & & & & & & & & & $\mathrm{X}$ & $\begin{array}{l}\text { N/A } \\
\text { No change. }\end{array}$ \\
\hline 9 & $\begin{array}{l}\text { Will not be able to } \\
\text { procure sufficient } \\
\text { extractant } \\
\text { quantities. }\end{array}$ & & & & & $\bar{x}$ & & & & & $\mathrm{X}$ & & $\begin{array}{l}\text { No cost impact or } \\
\text { schedule impact. } \\
\text { No change. }\end{array}$ \\
\hline
\end{tabular}




\begin{tabular}{|c|c|c|c|c|c|c|c|c|c|c|c|c|c|}
\hline \multirow{2}{*}{$\begin{array}{l}\text { ID } \\
\text { No }\end{array}$} & \multirow{2}{*}{$\begin{array}{c}\text { Caustic Side } \\
\text { Solvent Extraction } \\
\text { Uncertainty } \\
\text { Statement }\end{array}$} & \multicolumn{9}{|c|}{$\begin{array}{c}\text { Areas of } \\
\text { Uncertainty }\end{array}$} & \multirow[b]{2}{*}{1} & \multirow[b]{2}{*}{2} & \multirow[b]{2}{*}{ Explanatory Notes } \\
\hline & & Mission & $\begin{array}{l}\text { Technical } \\
\text { Maturity }\end{array}$ & Environmental & $\begin{array}{c}\text { Engineering/ } \\
\text { Design }\end{array}$ & Operation & Regulatory & $\begin{array}{l}\text { Stakeholder } \\
\end{array}$ & $\overline{\text { Safety }}$ & Radiological & & & \\
\hline 10 & $\begin{array}{l}\text { Public acceptability } \\
\text { may not be } \\
\text { achieved. }\end{array}$ & & & & & & & $\mathrm{x}$ & & & $\mathrm{X}$ & & $\begin{array}{l}\$ 500,000 \text { cost } \\
\text { increase for public } \\
\text { relations and } \\
\text { analysis. } \\
\text { No change. }\end{array}$ \\
\hline 11 & $\begin{array}{l}\text { DOE independent } \\
\text { project review and } \\
\text { acceptance may } \\
\text { impact project } \\
\text { milestones. }\end{array}$ & & & & & & $\bar{x}$ & & & & $X$ & & $\begin{array}{l}\text { Schedule impact of } \\
1 \text { month at end of } \\
\text { conceptual design, } \\
1 \text { month at the end } \\
\text { of preliminary } \\
\text { design, } 2 \text { month at } \\
\text { the end of final } \\
\text { design and } 1 \text { month } \\
\text { prior to radioactive } \\
\text { operations. } \\
\text { No change. }\end{array}$ \\
\hline
\end{tabular}




\begin{tabular}{|c|c|c|c|c|c|c|c|c|c|c|c|c|c|}
\hline \multirow{2}{*}{$\begin{array}{l}\text { ID } \\
\text { No }\end{array}$} & \multirow{2}{*}{$\begin{array}{c}\text { Caustic Side } \\
\text { Solvent Extraction } \\
\text { Uncertainty } \\
\text { Statement } \\
\end{array}$} & \multicolumn{9}{|c|}{$\begin{array}{c}\text { Areas of } \\
\text { Uncertainty }\end{array}$} & \multirow[b]{2}{*}{1} & \multirow[b]{2}{*}{2} & \multirow[b]{2}{*}{ Explanatory Notes } \\
\hline & & Mission & $\begin{array}{l}\text { Technical } \\
\text { Maturity }\end{array}$ & Environmental & $\begin{array}{c}\text { Engineering / } \\
\text { Design }\end{array}$ & Operation & Regulatory & Stakeholder & $\begin{array}{l}\text { Safety } \\
\end{array}$ & $\begin{array}{l}\text { Radiological } \\
\end{array}$ & & & \\
\hline 12 & $\begin{array}{l}\text { Change in } \\
\text { requirements and } \\
\text { standards, such as } \\
\text { NRC licensing may } \\
\text { impact the cost and } \\
\text { schedule. }\end{array}$ & & & & & & $\mathrm{x}$ & & & & & $\mathrm{X}$ & $\begin{array}{l}18 \text { month delay to } \\
\text { radioactive } \\
\text { operations. } \\
\text { Additional } \$ 1 \\
\text { million cost. SAR } \\
\text { may cause } 4 \text { month } \\
\text { delay in completing } \\
\text { preliminary design. } \\
\text { NRC standards } \\
\text { equivalency will be } \\
\text { part of design } \\
\text { process. } \\
\text { HLW-SDT-99. } \\
\text { 0062 } \\
\text { No change. }\end{array}$ \\
\hline 13 & $\begin{array}{l}\text { DOE lack of } \\
\text { support of required } \\
\text { budget and } \\
\text { schedule may delay } \\
\text { new facility startup. }\end{array}$ & & & & & & & $\mathrm{x}$ & & & $\mathrm{X}$ & & $\begin{array}{l}6 \text { month schedule } \\
\text { impact in the first } \\
\text { year. } \\
7 \text { month schedule } \\
\text { impact in the } \\
\text { second year. } \\
7 \text { month schedule } \\
\text { impact in the third } \\
\text { year. } \\
\text { No change. }\end{array}$ \\
\hline
\end{tabular}




\begin{tabular}{|c|c|c|c|c|c|c|c|c|c|c|c|c|c|}
\hline \multirow{2}{*}{$\begin{array}{l}\text { ID } \\
\text { No }\end{array}$} & \multirow{2}{*}{$\begin{array}{c}\text { Caustic Side } \\
\text { Solvent Extraction } \\
\text { Uncertainty } \\
\text { Statement }\end{array}$} & \multicolumn{9}{|c|}{$\begin{array}{c}\text { Areas of } \\
\text { Uncertainty }\end{array}$} & \multirow[b]{2}{*}{1} & \multirow[b]{2}{*}{2} & \multirow[b]{2}{*}{ Explanatory Notes } \\
\hline & & Mission & $\begin{array}{l}\text { Technical } \\
\text { Maturity }\end{array}$ & Environmental & $\begin{array}{c}\text { Engineering / } \\
\text { Design }\end{array}$ & Operation & Regulatory & Stakeholder & Safety & Radiological & & & \\
\hline 14 & $\begin{array}{l}\text { SRS infrastructure } \\
\text { may not support the } \\
\text { project needs. }\end{array}$ & & & & & $\mathrm{x}$ & & & & & $\mathrm{X}$ & & $\begin{array}{l}\$ 31 \text { million cost } \\
\text { increase for } \\
\text { overtime resulting } \\
\text { from staffing } \\
\text { delays. } \\
\text { No change. }\end{array}$ \\
\hline 15 & $\begin{array}{l}\text { Waste removal is } \\
\text { being accelerated. } \\
\text { May conflict with } \\
\text { preferred } \\
\text { alternatives or } \\
\text { preferred } \\
\text { alternative may } \\
\text { divert resources } \\
\text { from waste removal } \\
\text { project. }\end{array}$ & $\bar{x}$ & & & & & & & & & $\mathrm{X}$ & & $\begin{array}{l}\text { No additional cost } \\
\text { or schedule impact } \\
\text { beyond item } 13 . \\
\text { No change. }\end{array}$ \\
\hline
\end{tabular}




\begin{tabular}{|c|c|c|c|c|c|c|c|c|c|c|c|c|c|}
\hline \multirow{2}{*}{$\begin{array}{l}\text { ID } \\
\text { No }\end{array}$} & \multirow{2}{*}{$\begin{array}{c}\text { Caustic Side } \\
\text { Solvent Extraction } \\
\text { Uncertainty } \\
\text { Statement } \\
\end{array}$} & \multicolumn{9}{|c|}{$\begin{array}{c}\text { Areas of } \\
\text { Uncertainty }\end{array}$} & \multirow[b]{2}{*}{1} & \multirow[b]{2}{*}{2} & \multirow[b]{2}{*}{ Explanatory Notes } \\
\hline & & Mission & $\begin{array}{l}\text { Technical } \\
\text { Maturity }\end{array}$ & Environmental & $\begin{array}{c}\text { Engineering / } \\
\text { Design }\end{array}$ & Operation & $\begin{array}{l}\text { Regulatory } \\
\end{array}$ & Stakeholder & $\begin{array}{l}\text { Safety } \\
\end{array}$ & Radiological & & & \\
\hline 16 & $\begin{array}{l}\text { Pressure on 'old' } \\
\text { infrastructure will } \\
\text { increase, } \\
\text { endangering } \\
\text { schedule due to } \\
\text { three fold increase } \\
\text { in flow } \\
\text { requirements from } \\
\text { HTF and FTF. This } \\
\text { would endanger } \\
\text { performance of } \\
\text { infrastructure. }\end{array}$ & & & & & $\mathrm{x}$ & & & & & $X$ & & $\begin{array}{l}9 \text { month delay in } \\
\text { completing salt } \\
\text { removal from a } \\
\text { production schedule } \\
\text { delay to reach salt } \\
\text { solution feed rate } \\
\text { assumption. Basis: } \\
50 \% \text { material } \\
\text { movement in the } \\
\text { first year results in } \\
6 \text { months and } 75 \% \\
\text { material movement } \\
\text { in the second year } \\
\text { results in } 3 \text { months. } \\
\text { No change. }\end{array}$ \\
\hline 17 & $\begin{array}{l}\text { Improper contract } \\
\text { strategy for design } \\
\text { work may impact } \\
\text { the schedule. }\end{array}$ & & & & $\bar{x}$ & & & & & & $X$ & & $\begin{array}{l}6 \text { month delay in } \\
\text { completing } \\
\text { conceptual design. } \\
\text { No change. }\end{array}$ \\
\hline 18 & $\begin{array}{l}\text { Research and } \\
\text { development work } \\
\text { performed must be } \\
\text { coordinated with } \\
\text { the design effort. }\end{array}$ & & $\mathrm{x}$ & & & & & & & & $X$ & & $\begin{array}{l}3 \text { month delay in } \\
\text { completing } \\
\text { preliminary design. } \\
\text { No change. }\end{array}$ \\
\hline
\end{tabular}




\begin{tabular}{|c|c|c|c|c|c|c|c|c|c|c|c|c|c|}
\hline \multirow{2}{*}{$\begin{array}{l}\text { ID } \\
\text { No }\end{array}$} & \multirow{2}{*}{$\begin{array}{c}\text { Caustic Side } \\
\text { Solvent Extraction } \\
\text { Uncertainty } \\
\text { Statement }\end{array}$} & \multicolumn{9}{|c|}{$\begin{array}{c}\text { Areas of } \\
\text { Uncertainty }\end{array}$} & \multirow[b]{2}{*}{1} & \multirow[b]{2}{*}{2} & \multirow[b]{2}{*}{ Explanatory Notes } \\
\hline & & Mission & $\begin{array}{l}\text { Technical } \\
\text { Maturity }\end{array}$ & Environmental & $\begin{array}{c}\text { Engineering / } \\
\text { Design }\end{array}$ & Operation & Regulatory & Stakeholder & Safety & Radiological & & & \\
\hline 19 & $\begin{array}{l}\text { Geotechnical } \\
\text { problems with } \\
\text { siting locations } \\
\text { may cause schedule } \\
\text { delays. }\end{array}$ & & & r & $\mathrm{x}$ & & & & & & & $X$ & $\begin{array}{l}\text { Site selection and } \\
\text { geotechnical } \\
\text { characterization } \\
\text { was completed with } \\
\text { no subsurface } \\
\text { concerns. } \\
\text { WSRC-RP-99- } \\
00513\end{array}$ \\
\hline 20 & $\begin{array}{l}\text { A clearly defined } \\
\text { safety strategy } \\
\text { should be agreed to } \\
\text { by the end of } \\
\text { conceptual design } \\
\text { to preclude } \\
\text { schedule impacts. }\end{array}$ & & & & & & & & $\mathrm{x}$ & & $X$ & & $\begin{array}{l}2 \text { month delay in } \\
\text { start of preliminary } \\
\text { design. } \\
\text { No change. }\end{array}$ \\
\hline 21 & $\begin{array}{l}\text { High source term } \\
\text { with credible } \\
\text { release mechanisms } \\
\text { will concern public. }\end{array}$ & & & & & & & $\mathrm{x}$ & & & $\mathrm{X}$ & & $\begin{array}{l}\text { Within the existing } \\
\text { estimate. } \\
\text { No change. }\end{array}$ \\
\hline
\end{tabular}




\begin{tabular}{|c|c|c|c|c|c|c|c|c|c|c|c|c|c|}
\hline \multirow{2}{*}{$\begin{array}{l}\text { ID } \\
\text { No }\end{array}$} & \multirow{2}{*}{$\begin{array}{c}\text { Caustic Side } \\
\text { Solvent Extraction } \\
\text { Uncertainty } \\
\text { Statement }\end{array}$} & \multicolumn{9}{|c|}{$\begin{array}{c}\text { Areas of } \\
\text { Uncertainty }\end{array}$} & \multirow[b]{2}{*}{1} & \multirow[b]{2}{*}{2} & \multirow[b]{2}{*}{ Explanatory Notes } \\
\hline & & Mission & $\begin{array}{l}\text { Technical } \\
\text { Maturity }\end{array}$ & Environmental & $\begin{array}{c}\text { Engineering } \\
\text { Design }\end{array}$ & Operation & Regulatory & Stakeholder & Safety & Radiological & & & \\
\hline 22 & $\begin{array}{l}\text { Solvent estimated } \\
\text { unit cost rate may } \\
\text { be reduced. }\end{array}$ & & & & & $\mathrm{x}$ & & & & & $\mathrm{X}$ & & $\begin{array}{l}\text { Solvent extractant } \\
\text { cost bases } \\
\text { decreases from } \\
\$ 500 \text { to } \$ 175 \text { per } \\
\text { gram, resulting in a } \\
\$ 190 \text { million life } \\
\text { cycle cost decrease. } \\
\text { No change. }\end{array}$ \\
\hline 23 & $\begin{array}{l}\text { Solvent estimated } \\
\text { consumption cost } \\
\text { may be reduced. }\end{array}$ & & & & & $x$ & & & & & $\mathrm{X}$ & & $\begin{array}{l}\text { Change cost bases } \\
\text { to complete } \\
\text { replacement of } \\
\text { solvent every } 2 \\
\text { years and solvent } \\
\text { extractant cost } \\
\text { bases to } \$ 175 \text { per } \\
\text { gram resulting in a } \\
\$ 51 \text { million cost } \\
\text { decrease. } \\
\text { No change. }\end{array}$ \\
\hline 24 & $\begin{array}{l}\text { The interfacing } \\
\text { facilities } \\
\text { operational } \\
\text { schedules may } \\
\text { impact completion } \\
\text { of } \\
\text { tie-ins to the new } \\
\text { facility. }\end{array}$ & & & & & $\bar{x}$ & & & & & $\mathrm{X}$ & & $\begin{array}{l}2 \text { month production } \\
\text { delay for DWPF to } \\
\text { install new transfer } \\
\text { line. } \\
\text { No change. }\end{array}$ \\
\hline
\end{tabular}




\begin{tabular}{|c|c|c|c|c|c|c|c|c|c|c|c|c|c|}
\hline \multirow{2}{*}{$\begin{array}{l}\text { ID } \\
\text { No }\end{array}$} & \multirow{2}{*}{$\begin{array}{c}\text { Caustic Side } \\
\text { Solvent Extraction } \\
\text { Uncertainty } \\
\text { Statement }\end{array}$} & \multicolumn{9}{|c|}{$\begin{array}{c}\text { Areas of } \\
\text { Uncertainty }\end{array}$} & \multirow[b]{2}{*}{1} & \multirow[b]{2}{*}{2} & \multirow[b]{2}{*}{ Explanatory Notes } \\
\hline & & Mission & $\begin{array}{l}\text { Technical } \\
\text { Maturity }\end{array}$ & Environmental & $\begin{array}{c}\text { Engineering/ } \\
\text { Design }\end{array}$ & Operation & Regulatory & $\begin{array}{l}\text { Stakeholder } \\
\text { Sal }\end{array}$ & Safety & $\begin{array}{l}\text { Radiological } \\
\end{array}$ & & & \\
\hline 25 & $\begin{array}{l}\text { GT-73 unit } \\
\text { operations may not } \\
\text { be required. }\end{array}$ & & & & $\mathrm{x}$ & & & & & & $X$ & & $\begin{array}{l}\$ 25 \text { million cost } \\
\text { decrease. } \\
\text { No change. } \\
\text { WSRC-RP-99- } \\
00006\end{array}$ \\
\hline 26 & $\begin{array}{l}\text { Improved stripping } \\
\text { capabilities may } \\
\text { reduce the number } \\
\text { of overall stages by } \\
12 \text { to } 16 .\end{array}$ & & & & $\mathrm{x}$ & & & & & & $\mathrm{X}$ & & $\begin{array}{l}\$ 25 \text { million cost } \\
\text { decrease. The } \\
\text { addition of TOA } \\
\text { has reduced the } \\
\text { stripping coefficient } \\
\text { distribution. } \\
\text { HLW-SDT-99- } \\
0283\end{array}$ \\
\hline 27 & $\begin{array}{l}\text { Difficulty in } \\
\text { resuspending MST } \\
\text { after long quiescent } \\
\text { period will require } \\
\text { temperature control } \\
\text { and mixing } \\
\text { equipment. }\end{array}$ & & & & $\mathrm{x}$ & & & & & & $\mathrm{X}$ & & $\begin{array}{l}\$ 10 \text { million cost } \\
\text { increase (based on } \\
\text { the cost estimate for } \\
\text { a } 100 \mathrm{Kgal} \text {. Alpha } \\
\text { adsorption tank). } \\
\text { ORNL/TM- } \\
1999 / 166\end{array}$ \\
\hline
\end{tabular}




\subsubsection{CST Non-Elutable Ion Exchange}

\begin{tabular}{|c|c|c|c|c|c|c|c|c|c|c|c|c|c|}
\hline \multirow{2}{*}{$\begin{array}{l}\text { ID } \\
\text { No }\end{array}$} & \multirow{2}{*}{$\begin{array}{l}\text { CST Non-Elutable } \\
\text { Ion Exchange } \\
\text { Uncertainty } \\
\text { Statement }\end{array}$} & \multicolumn{9}{|c|}{$\begin{array}{l}\text { Areas of } \\
\text { Uncertainty }\end{array}$} & \multirow[b]{2}{*}{1} & \multirow[b]{2}{*}{2} & \multirow[b]{2}{*}{ Explanatory Notes } \\
\hline & & $\begin{array}{c}\text { Mission } \\
\end{array}$ & $\begin{array}{l}\text { Technical } \\
\text { Maturity }\end{array}$ & \begin{tabular}{|l|l} 
Environmental \\
\end{tabular} & $\begin{array}{c}\text { Enginering/ } \\
\text { Design }\end{array}$ & $\begin{array}{l}\text { Operation } \\
\text { nat }\end{array}$ & 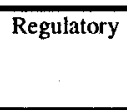 & Stakeholder & $\begin{array}{l}\text { Safety } \\
\end{array}$ & $\begin{array}{l}\text { Radiological } \\
\end{array}$ & & & \\
\hline 1 & $\begin{array}{l}\text { What is the fate of } \\
\text { Am in the process? }\end{array}$ & & $\mathrm{x}$ & & & & & & & & & $\mathrm{X}$ & $\begin{array}{l}\text { N/A } \\
\text { No change. }\end{array}$ \\
\hline 2 & $\begin{array}{l}\text { Inability to remove } \\
\text { spent resin from a } \\
\text { column. }\end{array}$ & & & & $\bar{x}$ & & & & & & & $\mathrm{X}$ & $\begin{array}{l}\text { Resin transport has } \\
\text { been demonstrated. } \\
\text { No change. } \\
\text { ORNL-TM-1999/103 }\end{array}$ \\
\hline 3 & $\begin{array}{l}\text { Method of flow } \\
\text { control between } \\
\text { columns } \\
\text { (pumping/gravity) }\end{array}$ & & & & $x$ & & & & & & & $\mathrm{X}$ & $\begin{array}{l}\text { Demonstrated } \\
\text { down-flow } \\
\text { configuration with } \\
\text { gas disengagement } \\
\text { from the bottom of } \\
\text { the column. } \\
\text { No change. } \\
\text { ORNL-TM-1999/103 }\end{array}$ \\
\hline
\end{tabular}




\begin{tabular}{|c|c|c|c|c|c|c|c|c|c|c|c|c|c|}
\hline \multirow{2}{*}{$\begin{array}{l}\text { ID } \\
\text { No }\end{array}$} & \multirow{2}{*}{$\begin{array}{c}\text { CST Non-Elutable } \\
\text { Ion Exchange } \\
\text { Uncertainty } \\
\text { Statement } \\
\end{array}$} & \multicolumn{9}{|c|}{$\begin{array}{c}\text { Areas of } \\
\text { Uncertainty }\end{array}$} & \multirow[b]{2}{*}{1} & \multirow[b]{2}{*}{2} & \multirow[b]{2}{*}{ Explanatory Notes } \\
\hline & & Mission & $\begin{array}{l}\text { Technical } \\
\text { Maturity }\end{array}$ & Environmental & $\begin{array}{l}\text { Engincering / } \\
\text { Design }\end{array}$ & Operation & Regulatory & Stakeholder & Safety & Radiological & & & \\
\hline 4 & $\begin{array}{l}\text { Resin bed } \\
\text { temperature control } \\
\text { during operational } \\
\text { conditions and } \\
\text { loaded spent resin } \\
\text { temperature } \\
\text { control. }\end{array}$ & & & . & $\mathrm{x}$ & & ${ }^{\circ}$ & & & & $\mathrm{X}$ & & $\begin{array}{l}\$ 10 \text { million cost } \\
\text { increase for safety } \\
\text { class emergency } \\
\text { cooling and } \\
\text { temperature } \\
\text { monitoring. R\&D } \\
\text { results indicate a } \\
\text { need for cooling to } \\
\text { support normal and } \\
\text { emergency } \\
\text { operations. } \\
\text { No change. } \\
\text { ORNL-TM-1999/233 }\end{array}$ \\
\hline 5 & $\begin{array}{l}\text { Analysis delay (1 } \\
\text { week) in measuring } \\
\text { for Sr DF in MST } \\
\text { process. }\end{array}$ & & $\mathrm{x}$ & & & & & & & & $X$ & & $\begin{array}{l}\text { No cost or schedule } \\
\text { impact, within } \\
\text { existing R\&D } \\
\text { scope and schedule } \\
\text { duration. } \\
\text { No change. }\end{array}$ \\
\hline
\end{tabular}




\begin{tabular}{|c|c|c|c|c|c|c|c|c|c|c|c|c|c|}
\hline \multirow{2}{*}{$\begin{array}{l}\text { ID } \\
\text { No }\end{array}$} & \multirow{2}{*}{$\begin{array}{l}\text { CST Non-Elutable } \\
\text { Ion Exchange } \\
\text { Uncertainty } \\
\text { Statement }\end{array}$} & \multicolumn{9}{|c|}{$\begin{array}{c}\text { Areas of } \\
\text { Uncertainty }\end{array}$} & \multirow[b]{2}{*}{1} & \multirow[b]{2}{*}{2} & \multirow[b]{2}{*}{ Explanatory Notes } \\
\hline & & Mission & $\begin{array}{l}\text { Technical } \\
\text { Maturity }\end{array}$ & Environmental & $\begin{array}{c}\text { Engineering / } \\
\text { Design }\end{array}$ & Operation & Regulatory & Stakeholder & Safety & Radiological & & & \\
\hline 6 & $\begin{array}{l}\text { Can pressure } \\
\text { gradients crush the } \\
\text { resin during column } \\
\text { operations? }\end{array}$ & & . & & $\mathrm{x}$ & & & & & & $X$ & & $\begin{array}{l}\$ 2.5 \text { million cost } \\
\text { increase for } 4 \\
\text { additional columns. } \\
\$ 2.5 \text { million cost } \\
\text { increase for } \\
\text { associated jumpers. } \\
\$ 2.6 \text { million cost } \\
\text { increase for } 2 \\
\text { additional } \\
\text { personnel during } \\
\text { the operational life } \\
\text { of the facility. } \\
\text { No change. }\end{array}$ \\
\hline 7 & $\begin{array}{l}\text { Application of } \\
\text { carousel design in a } \\
\text { remote environment } \\
\text { (e.g. operation and } \\
\text { maintenance). }\end{array}$ & & & & $\mathrm{X}$ & & & & & & & $\mathrm{X}$ & $\begin{array}{l}\text { Design incorporates } \\
\text { jumpers and not } \\
\text { valves. } \\
\text { No change. }\end{array}$ \\
\hline 8 & $\begin{array}{l}\text { Is the shielding in } \\
\text { the current transfer } \\
\text { lines adequate for } \\
\text { transferring spent/ } \\
\text { loaded resin? }\end{array}$ & & & & & & & & & $\bar{x}$ & & $\mathrm{X}$ & $\begin{array}{l}\text { N/A } \\
\text { No change. }\end{array}$ \\
\hline
\end{tabular}




\begin{tabular}{|c|c|c|c|c|c|c|c|c|c|c|c|c|c|}
\hline \multirow{2}{*}{$\begin{array}{l}\text { ID } \\
\text { No }\end{array}$} & \multirow{2}{*}{$\begin{array}{l}\text { CST Non-Elutable } \\
\text { Ion Exchange } \\
\text { Uncertainty } \\
\text { Statement }\end{array}$} & \multicolumn{9}{|c|}{$\begin{array}{c}\text { Areas of } \\
\text { Uncertainty }\end{array}$} & \multirow[b]{2}{*}{1} & \multirow[b]{2}{*}{2} & \multirow[b]{2}{*}{ Explanatory Notes } \\
\hline & & Mission & $\begin{array}{l}\text { Technical } \\
\text { Maturity }\end{array}$ & Environmental & $\begin{array}{c}\text { Engineering / } \\
\text { Design }\end{array}$ & Operation & Regulatory & Stakeholder & $\begin{array}{l}\text { Safety } \\
\end{array}$ & Radiological & & & \\
\hline 9 & $\begin{array}{l}\text { Difficulty in } \\
\text { filtration of sludge } \\
\text { and/or MST will } \\
\text { produce low filtrate } \\
\text { flow rates and } \\
\text { require frequent } \\
\text { cleaning }\end{array}$ & & & & $\mathrm{X}$ & & & & & & $X$ & & $\begin{array}{l}\$ 6 \text { million cost } \\
\text { increase for the } \\
\text { larger filters. } \\
\$ 10 \text { million cost } \\
\text { increase for the } \\
\text { larger pumps. Flux } \\
\text { rate decrease from } \\
0.07 \text { to } 0.02 \text {. } \\
\text { WSRC-TR-99- } \\
0342\end{array}$ \\
\hline 10 & $\begin{array}{l}\text { TRU } \\
\text { decontamination } \\
\text { with MST is not } \\
\text { adequate with the } \\
\text { design residence } \\
\text { time. }\end{array}$ & & $\mathrm{x}$ & & & & & & & & $X$ & & $\begin{array}{l}125 \mathrm{Kgal} \text { double } \\
\text { lobe tank for } \\
\text { dilution effect. } \\
\text { MST concentration } \\
\text { and residence time } \\
\text { requires more } \\
\text { research for } \\
\text { bounding waste. No } \\
\text { additional cost } \\
\text { beyond Item } 34 \text {. }\end{array}$ \\
\hline
\end{tabular}




\begin{tabular}{|c|c|c|c|c|c|c|c|c|c|c|c|c|c|}
\hline \multirow{2}{*}{$\begin{array}{l}\text { ID } \\
\text { No }\end{array}$} & \multirow{2}{*}{$\begin{array}{l}\text { CST Non-Elutable } \\
\text { Ion Exchange } \\
\text { Uncertainty } \\
\text { Statement }\end{array}$} & \multicolumn{9}{|c|}{$\begin{array}{c}\text { Areas of } \\
\text { Uncertainty }\end{array}$} & \multirow[b]{2}{*}{1} & \multirow[b]{2}{*}{2} & \multirow[b]{2}{*}{ Explanatory Notes } \\
\hline & & Mission & $\begin{array}{l}\text { Technical } \\
\text { Maturity }\end{array}$ & Environmental & $\begin{array}{l}\text { Engineering / } \\
\text { Design }\end{array}$ & Operation & Regulatory & Stakeholder & $\begin{array}{l}\text { Safety } \\
\end{array}$ & $\overline{\text { Radiological }}$ & & & \\
\hline 11 & $\begin{array}{l}\text { Process chemistry } \\
\text { understanding and } \\
\text { application are still } \\
\text { under development, } \\
\text { resulting in 96-1 } \\
\text { lessons learned not } \\
\text { yet implemented }\end{array}$ & & $\mathrm{x}$ & 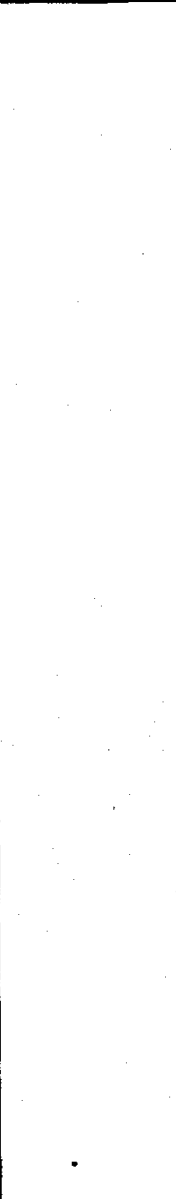 & & & & & & & $X$ & & $\begin{array}{l}9 \text { month delay in } \\
\text { completing } \\
\text { conceptual design } \\
\text { and a } 9 \text { month } \\
\text { delay in completing } \\
\text { preliminary design. } \\
\$ 10 \text { million cost } \\
\text { increase to support } \\
\text { product } \\
\text { development. } \\
\text { Product is } \\
\text { considered to be } \\
\text { still under } \\
\text { development and } \\
\text { by experiment, has } \\
\text { exhibited stability } \\
\text { and leaching } \\
\text { problems. A } 1 \text { to } 2 \\
\text { year development } \\
\text { duration has been } \\
\text { suggested by the } \\
\text { developer and the } \\
\text { vendor. } \\
\text { ORNL/TM- } \\
1999 / 233\end{array}$ \\
\hline
\end{tabular}


Page 160 of 277

\begin{tabular}{|c|c|c|c|c|c|c|c|c|c|c|c|c|c|}
\hline \multirow{2}{*}{$\begin{array}{l}\text { ID } \\
\text { No }\end{array}$} & \multirow{2}{*}{$\begin{array}{c}\text { CST Non-Elutable } \\
\text { Ion Exchange } \\
\text { Uncertainty } \\
\text { Statement } \\
\end{array}$} & \multicolumn{9}{|c|}{$\begin{array}{c}\text { Areas of } \\
\text { Uncertainty }\end{array}$} & \multirow[b]{2}{*}{1} & \multirow[b]{2}{*}{2} & \multirow[b]{2}{*}{ Explanatory Notes } \\
\hline & & Mission & $\begin{array}{l}\text { Technical } \\
\text { Maturity }\end{array}$ & Environmental & $\begin{array}{c}\text { Engineering / } \\
\text { Design }\end{array}$ & Operation & Regulatory & Stakeholder & Safety & Radiological & & & \\
\hline 12 & $\begin{array}{l}\text { CST will require } \\
\text { "requalification" of } \\
\text { glass form. }\end{array}$ & & & & & & $\bar{x}$ & & & & $\mathrm{X}$ & & $\begin{array}{l}\$ 15 \text { million cost } \\
\text { increase to support } \\
\text { glass requalification } \\
(\text { durability and } \\
\left.\mathrm{TiO}_{2}\right) \text {. R\&D has } \\
\text { indicated glass } \\
\text { requalification and } \\
\text { hydraguard } \\
\text { sampling } \\
\text { modification is } \\
\text { required. } \\
\text { WSRC-RP-99- } \\
\text { 00195 } \\
\text { WSRC-TR-99- } \\
\text { 00245 }\end{array}$ \\
\hline
\end{tabular}




\begin{tabular}{|c|c|c|c|c|c|c|c|c|c|c|c|c|c|}
\hline \multirow{2}{*}{$\begin{array}{l}\text { ID } \\
\text { No }\end{array}$} & \multirow{2}{*}{$\begin{array}{l}\text { CST Non-Elutable } \\
\text { Ion Exchange } \\
\text { Uncertainty } \\
\text { Statement }\end{array}$} & \multicolumn{9}{|c|}{$\begin{array}{c}\text { Areas of } \\
\text { Uncertainty }\end{array}$} & \multirow[b]{2}{*}{1} & \multirow[b]{2}{*}{2} & \multirow[b]{2}{*}{ Explanatory Notes } \\
\hline & & Mission & $\begin{array}{l}\text { Technical } \\
\text { Maturity }\end{array}$ & Environmental & 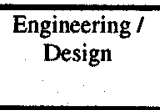 & Operation & Regulatory & Stakeholder & Safety & Radiological & & & \\
\hline 13 & $\begin{array}{l}\text { The CST material } \\
\text { may not be } \\
\text { available in } \\
\text { sufficient quantities } \\
\text { to support the } \\
\text { process ( } 50 \text { tons per } \\
\text { year). }\end{array}$ & & & & & $\bar{x}$ & & & & & $\mathrm{X}$ & & $\begin{array}{l}\text { No schedule impact } \\
\text { and within the cost } \\
\text { estimate. Scale up } \\
\text { attempts (2) have } \\
\text { resulted in issues to } \\
\text { be resolved. } \\
\text { No change. } \\
\text { WSRC-RP-99- } \\
\text { 00568 }\end{array}$ \\
\hline 14 & $\begin{array}{l}\text { Major sample } \\
\text { station modification } \\
\text { affecting DWPF } \\
\text { operations. }\end{array}$ & & & & & & & & & $\mathrm{X}$ & $X$ & & $\begin{array}{l}\$ 5 \text { million cost } \\
\text { increase for sample } \\
\text { cell modifications } \\
\text { for shielding. } \\
\text { No change. }\end{array}$ \\
\hline 15 & $\begin{array}{l}\text { CST resin fines } \\
\text { may collect in } \\
\text { downstream filters, } \\
\text { elbows, imperfect } \\
\text { welds, and } \\
\text { instrument lines. }\end{array}$ & & & & & & & & & $x$ & $X$ & & $\begin{array}{l}\$ 2 \text { million cost } \\
\text { increase for related } \\
\text { modifications (e.g., } \\
\text { shielding). } \\
\text { No change. }\end{array}$ \\
\hline
\end{tabular}




\begin{tabular}{|c|c|c|c|c|c|c|c|c|c|c|c|c|c|}
\hline \multirow{2}{*}{$\begin{array}{l}\text { ID } \\
\text { No }\end{array}$} & \multirow{2}{*}{$\begin{array}{c}\text { CST Non-Elutable } \\
\text { Ion Exchange } \\
\text { Uncertainty } \\
\text { Statement }\end{array}$} & \multicolumn{9}{|c|}{$\begin{array}{c}\text { Areas of } \\
\text { Uncertainty }\end{array}$} & \multirow[b]{2}{*}{1} & \multirow[b]{2}{*}{2} & \multirow[b]{2}{*}{ Explanatory Notes } \\
\hline & & Mission & $\begin{array}{l}\text { Technical } \\
\text { Maturity }\end{array}$ & Environmental & $\begin{array}{c}\text { Engineering/ } \\
\text { Design }\end{array}$ & Operation & Regulatory & Stakcholder & Safety & Radiological & & & \\
\hline 16 & $\begin{array}{l}\text { DOE independent } \\
\text { project review and } \\
\text { acceptance may } \\
\text { impact project } \\
\text { milestones. }\end{array}$ & & & & & & $\mathrm{X}$ & & & & $\mathrm{X}$ & & $\begin{array}{l}\text { Schedule impact of } \\
1 \text { month at end of } \\
\text { conceptual design, } \\
1 \text { month at the end } \\
\text { of preliminary } \\
\text { design, } 2 \text { months at } \\
\text { the end of final } \\
\text { design and } 1 \text { month } \\
\text { prior to radioactive } \\
\text { operations. } \\
\text { No change. }\end{array}$ \\
\hline
\end{tabular}




\begin{tabular}{|c|c|c|c|c|c|c|c|c|c|c|c|c|c|}
\hline \multirow{2}{*}{$\begin{array}{l}\text { ID } \\
\text { No }\end{array}$} & \multirow{2}{*}{$\begin{array}{l}\text { CST Non-Elutable } \\
\text { Ion Exchange } \\
\text { Uncertainty } \\
\text { Statement }\end{array}$} & \multicolumn{9}{|c|}{$\begin{array}{c}\text { Areas of } \\
\text { Uncertainty }\end{array}$} & \multirow[b]{2}{*}{1} & \multirow[b]{2}{*}{2} & \multirow[b]{2}{*}{ Explanatory Notes } \\
\hline & & $\begin{array}{l}\text { Mission } \\
\end{array}$ & $\begin{array}{l}\text { Technical } \\
\text { Maturity }\end{array}$ & Environmental & $\begin{array}{c}\text { Engineering } \\
\text { Design }\end{array}$ & Operation & Regulatory & Stakeholder & $\begin{array}{l}\text { Safety } \\
\end{array}$ & Radiological & & & \\
\hline 17 & $\begin{array}{l}\text { Change in } \\
\text { requirements and } \\
\text { standards, such as } \\
\text { NRC licensing may } \\
\text { impact the cost and } \\
\text { schedule. }\end{array}$ & & & & & & $x$ & & & & $X$ & & $\begin{array}{l}18 \text { month delay to } \\
\text { radioactive } \\
\text { operations. } \\
\text { Additional } \$ 1 \\
\text { million cost. SAR } \\
\text { may cause } 4 \text { month } \\
\text { delay in completing } \\
\text { preliminary design. } \\
\text { NRC standards } \\
\text { equivalency will be } \\
\text { part of the design } \\
\text { process. } \\
\text { HLW-SDT-99- } \\
0062 \\
\text { No change. }\end{array}$ \\
\hline 18 & $\begin{array}{l}\text { The interfacing } \\
\text { facilities } \\
\text { operational } \\
\text { schedules may } \\
\text { impact completion } \\
\text { of tie-ins to the new } \\
\text { facility. }\end{array}$ & & & & & $\mathrm{x}$ & & & & & $\mathrm{X}$ & & $\begin{array}{l}2 \text { month production } \\
\text { delay for DWPF to } \\
\text { install new transfer } \\
\text { line. } \\
\text { No change. }\end{array}$ \\
\hline
\end{tabular}




\begin{tabular}{|c|c|c|c|c|c|c|c|c|c|c|c|c|c|}
\hline \multirow{2}{*}{$\begin{array}{l}\text { ID } \\
\text { No }\end{array}$} & \multirow{2}{*}{$\begin{array}{c}\text { CST Non-Elutable } \\
\text { Ion Exchange } \\
\text { Uncertainty } \\
\text { Statement }\end{array}$} & \multicolumn{9}{|c|}{$\begin{array}{c}\text { Areas of } \\
\text { Uncertainty }\end{array}$} & \multirow[b]{2}{*}{1} & \multirow[b]{2}{*}{2} & \multirow[b]{2}{*}{ Explanatory Notes } \\
\hline & & Mission & $\begin{array}{l}\text { Technical } \\
\text { Maturity }\end{array}$ & Environmental & $\begin{array}{c}\text { Engineering / } \\
\text { Design }\end{array}$ & Operation & Regulatory & Stakeholder & Safety & Radiological & & & \\
\hline 19 & $\begin{array}{l}\text { DOE lack of } \\
\text { support of required } \\
\text { budget and } \\
\text { schedule may delay } \\
\text { new facility startup. }\end{array}$ & & & & & & & $\mathrm{x}$ & & & $\mathrm{X}$ & & $\begin{array}{l}5 \text { month schedule } \\
\text { impact in the first } \\
\text { year. } \\
5 \text { month schedule } \\
\text { impact in the } \\
\text { second year. } \\
4 \text { month schedule } \\
\text { impact in the third } \\
\text { year. } \\
\text { No change. }\end{array}$ \\
\hline 20 & $\begin{array}{l}\text { SRS infrastructure } \\
\text { may not support the } \\
\text { project needs. }\end{array}$ & & & & & $\mathrm{x}$ & & & & & $X$ & & $\begin{array}{l}\$ 26.5 \text { million cost } \\
\text { increase for } \\
\text { overtime resulting } \\
\text { from staffing } \\
\text { delays. } \\
\text { No change. }\end{array}$ \\
\hline
\end{tabular}


Page 165 of 277

\begin{tabular}{|c|c|c|c|c|c|c|c|c|c|c|c|c|c|}
\hline \multirow{2}{*}{$\begin{array}{l}\text { ID } \\
\text { No }\end{array}$} & \multirow{2}{*}{$\begin{array}{l}\text { CST Non-Elutable } \\
\text { Ion Exchange } \\
\text { Uncertainty } \\
\text { Statement }\end{array}$} & \multicolumn{9}{|c|}{$\begin{array}{c}\text { Areas of } \\
\text { Uncertainty }\end{array}$} & \multirow[b]{2}{*}{1} & \multirow[b]{2}{*}{2} & \multirow[b]{2}{*}{ Explanatory Notes } \\
\hline & & Mission & $\begin{array}{l}\text { Technical } \\
\text { Maturity }\end{array}$ & Environmental & $\begin{array}{c}\text { Engineering/ } \\
\text { Design }\end{array}$ & Operation & Regulatory & $\begin{array}{l}\text { Stakeholder } \\
\text { Sal }\end{array}$ & Safety & Radiological & & & \\
\hline 21 & $\begin{array}{l}\text { Waste removal is } \\
\text { being accelerated. } \\
\text { May conflict with } \\
\text { preferred } \\
\text { alternatives or } \\
\text { preferred } \\
\text { alternative may } \\
\text { divert resources } \\
\text { from waste removal } \\
\text { project. }\end{array}$ & $\mathrm{x}$ & & & & & & & & & $\mathrm{X}$ & & $\begin{array}{l}\text { No additional cost } \\
\text { and schedule } \\
\text { impact beyond Item } \\
19 . \\
\text { No change. }\end{array}$ \\
\hline 22 & $\begin{array}{l}\text { Pressure on 'old' } \\
\text { infrastructure will } \\
\text { increase, } \\
\text { endangering } \\
\text { schedule due to } \\
\text { three fold increase } \\
\text { in flow } \\
\text { requirements from } \\
\text { HTF and FTF. This } \\
\text { would endanger } \\
\text { performance of } \\
\text { infrastructure. }\end{array}$ & & & & & $\mathrm{x}$ & & & & & $X$ & & $\begin{array}{l}9 \text { month delay in } \\
\text { completing salt } \\
\text { removal from a } \\
\text { production schedule } \\
\text { delay to reach salt } \\
\text { solution feed rate } \\
\text { assumption. Basis: } \\
50 \% \text { material } \\
\text { movement in the } \\
\text { first year results in } \\
6 \text { months and } 75 \% \\
\text { material movement } \\
\text { in the second year } \\
\text { results in } 3 \text { months. } \\
\text { No change. }\end{array}$ \\
\hline
\end{tabular}




\begin{tabular}{|c|c|c|c|c|c|c|c|c|c|c|c|c|c|}
\hline \multirow{2}{*}{$\begin{array}{l}\text { D } \\
\text { No }\end{array}$} & \multirow{2}{*}{$\begin{array}{l}\text { CST Non-Elutable } \\
\text { Ion Exchange } \\
\text { Uncertainty } \\
\text { Statement }\end{array}$} & \multicolumn{9}{|c|}{$\begin{array}{c}\text { Areas of } \\
\text { Uncertainty }\end{array}$} & \multirow[b]{2}{*}{1} & \multirow[b]{2}{*}{2} & \multirow[b]{2}{*}{ Explanatory Notes } \\
\hline & & Mission & $\begin{array}{l}\text { Technical } \\
\text { Maturity }\end{array}$ & Environmental & $\begin{array}{l}\text { Engineering/ } \\
\text { Design }\end{array}$ & Operation & Regulatory & Stakeholder & Safety & Radiological & & & \\
\hline 23 & $\begin{array}{l}\text { Improper contract } \\
\text { strategy for design } \\
\text { work may impact } \\
\text { the schedule. }\end{array}$ & & & & $\bar{x}$ & & & & & & $\bar{X}$ & & $\begin{array}{l}6 \text { month delay in } \\
\text { completing } \\
\text { conceptual design. } \\
\text { No change. }\end{array}$ \\
\hline 24 & $\begin{array}{l}\text { Research and } \\
\text { development work } \\
\text { performed must be } \\
\text { coordinated with } \\
\text { the design effort. }\end{array}$ & & $\bar{x}$ & & & & & & & & $\mathrm{X}$ & & $\begin{array}{l}6 \text { month delay in } \\
\text { completing } \\
\text { preliminary design. } \\
\text { No change. }\end{array}$ \\
\hline 25 & $\begin{array}{l}\text { Geotechnical } \\
\text { problems with } \\
\text { siting locations may } \\
\text { cause schedule } \\
\text { delays. }\end{array}$ & & & & $\mathrm{x}$ & & & & & & & $\mathrm{X}$ & $\begin{array}{l}\text { Site selection and } \\
\text { geotechnical } \\
\text { characterization } \\
\text { was completed with } \\
\text { no subsurface } \\
\text { concerns. } \\
\text { WSRC-RP-99- } \\
00513\end{array}$ \\
\hline 26 & $\begin{array}{l}\text { A clearly defined } \\
\text { safety strategy } \\
\text { should be agreed to } \\
\text { by the end of } \\
\text { conceptual design } \\
\text { to preclude } \\
\text { schedule impacts. }\end{array}$ & & & & & & & & $\mathrm{x}$ & & $\bar{X}$ & & $\begin{array}{l}2 \text { month delay in } \\
\text { the start of } \\
\text { preliminary design. } \\
\text { No change. }\end{array}$ \\
\hline
\end{tabular}


Page 167 of 277

\begin{tabular}{|c|c|c|c|c|c|c|c|c|c|c|c|c|c|}
\hline \multirow{2}{*}{$\begin{array}{l}\text { D } \\
\text { No }\end{array}$} & \multirow{2}{*}{$\begin{array}{l}\text { CST Non-Elutable } \\
\text { Ion Exchange } \\
\text { Uncertainty } \\
\text { Statement }\end{array}$} & \multicolumn{9}{|c|}{$\begin{array}{c}\text { Areas of } \\
\text { Uncertainty }\end{array}$} & \multirow[b]{2}{*}{1} & \multirow[b]{2}{*}{2} & \multirow[b]{2}{*}{ Explanatory Notes } \\
\hline & & Mission & $\begin{array}{l}\text { Technical } \\
\text { Maturity }\end{array}$ & Environmental & $\begin{array}{c}\text { Engineering / } \\
\text { Design }\end{array}$ & Operation & Regulatory & Stakeholder & Safety & $\begin{array}{l}\text { Radiological } \\
\end{array}$ & & & \\
\hline 27 & $\begin{array}{l}\text { High source term } \\
\text { with credible } \\
\text { release mechanisms } \\
\text { will concern public. }\end{array}$ & & & & & & & & $\bar{x}$ & & $\mathrm{X}$ & & $\begin{array}{l}\text { Within the existing } \\
\text { estimate. } \\
\text { No change. }\end{array}$ \\
\hline 28 & $\begin{array}{l}\text { Increased foaming } \\
\text { in the DWPF } \\
\text { Chemical Process } \\
\text { Cell. }\end{array}$ & & $\mathrm{x}$ & & & & & & & & & $\mathrm{X}$ & $\begin{array}{l}\text { Foaming during } \\
1 / 240^{\text {th }} \text { scale testing } \\
\text { was acceptable and } \\
\text { no scale up issues } \\
\text { expected. } \\
\text { WSRC-TR-99- } \\
00302\end{array}$ \\
\hline 29 & $\begin{array}{l}\text { Cesium desorption } \\
\text { at elevated resin } \\
\text { temperature. }\end{array}$ & & $\mathrm{X}$ & & & & & & & & $\bar{X}$ & & $\begin{array}{l}\text { No additional cost } \\
\text { impact beyond Item } \\
4 . \\
\text { Cesium desorps at } \\
\text { elevated } \\
\text { temperature. } \\
\text { ORNLTM-1999/233 } \\
\text { No change. }\end{array}$ \\
\hline 30 & $\begin{array}{l}\text { GT- } 73 \text { unit } \\
\text { operation may not } \\
\text { be required. }\end{array}$ & & & & $\bar{x}$ & & & & & & $\bar{X}$ & & $\begin{array}{l}\$ 27 \text { million cost } \\
\text { decrease. } \\
\text { No change. } \\
\text { WSRC-RP-99- } \\
00006\end{array}$ \\
\hline
\end{tabular}




\begin{tabular}{|c|c|c|c|c|c|c|c|c|c|c|c|c|c|}
\hline \multirow{2}{*}{$\begin{array}{l}\text { DD } \\
\text { No }\end{array}$} & \multirow{2}{*}{$\begin{array}{l}\text { CST Non-Elutable } \\
\text { Ion Exchange } \\
\text { Uncertainty } \\
\text { Statement } \\
\end{array}$} & \multicolumn{9}{|c|}{$\begin{array}{c}\text { Areas of } \\
\text { Uncertainty }\end{array}$} & \multirow[b]{2}{*}{1} & \multirow[b]{2}{*}{2} & \multirow[b]{2}{*}{ Explanatory Notes } \\
\hline & & Mission & $\begin{array}{l}\text { Technical } \\
\text { Maturity }\end{array}$ & Environmental & $\begin{array}{c}\text { Engineering/ } \\
\text { Design }\end{array}$ & Operation & Regulatory & Stakeholder & Safety & Radiological & & & \\
\hline 31 & $\begin{array}{l}\text { Resin stability at } \\
\text { elevated } \\
\text { temperature. }\end{array}$ & & $\mathrm{x}$ & & & & & & & & $\mathrm{X}$ & & $\begin{array}{l}\text { No additional cost } \\
\text { impact beyond Item } \\
11 . \\
\text { No change. }\end{array}$ \\
\hline 32 & $\begin{array}{l}\text { Impact of CST on } \\
\text { DWPF redox. }\end{array}$ & & $\mathrm{x}$ & & & & & & & & $X$ & & $\begin{array}{l}\text { No additional } \\
\text { schedule impact } \\
\text { beyond } \\
\text { Item } 24 \text {. } \\
\text { No change. } \\
\end{array}$ \\
\hline 33 & $\begin{array}{l}\text { Hydrogen and } \\
\text { oxygen generation } \\
\text { in the loaded } \\
\text { column. }\end{array}$ & & & & $\mathrm{x}$ & & & & & & $\mathrm{X}$ & & $\begin{array}{l}\text { Tankage for } \\
\text { hydrogen gas } \\
\text { collection and } \\
\text { associated safety } \\
\text { equipment. } \\
\$ 30 \text { million cost } \\
\text { increase. Gas } \\
\text { generation was } \\
\text { demonstrated to } \\
\text { require gas } \\
\text { disengagement } \\
\text { equipment at the } \\
\text { outlet of the } \\
\text { column. } \\
\text { No change. }\end{array}$ \\
\hline
\end{tabular}




\begin{tabular}{|c|c|c|c|c|c|c|c|c|c|c|c|c|c|}
\hline \multirow{2}{*}{$\begin{array}{l}\text { ID } \\
\text { No }\end{array}$} & \multirow{2}{*}{$\begin{array}{l}\text { CST Non-Elutable } \\
\text { Ion Exchange } \\
\text { Uncertainty } \\
\text { Statement }\end{array}$} & \multicolumn{9}{|c|}{$\begin{array}{c}\text { Areas of } \\
\text { Uncertainty }\end{array}$} & \multirow[b]{2}{*}{1} & \multirow[b]{2}{*}{2} & \multirow[b]{2}{*}{ Explanatory Notes } \\
\hline & & Mission & $\begin{array}{l}\text { Technical } \\
\text { Maturity }\end{array}$ & Environmental & $\begin{array}{c}\text { Engineering } \\
\text { Design }\end{array}$ & Operation & Regulatory & Stakeholder & Safety & Radiological & & & \\
\hline 34 & $\begin{array}{l}\text { Difficulty in } \\
\text { resuspending MST } \\
\text { after long quiescent } \\
\text { period will require } \\
\text { temperature control } \\
\text { and mixing } \\
\text { equipment. }\end{array}$ & & & & $\mathrm{x}$ & & & & & & $\mathrm{X}$ & & $\begin{array}{l}\$ 10 \text { million cost } \\
\text { increase (based on } \\
\text { the cost estimate for } \\
\text { a } 100 \text { Kgal. Alpha } \\
\text { adsorption tank). } \\
\text { ORNL/TM- } \\
1999 / 166\end{array}$ \\
\hline 35 & $\begin{array}{l}\text { Aluminum } \\
\text { precipitation in the } \\
\text { resin column } \\
\text { impacts production. }\end{array}$ & & & & $\mathrm{x}$ & & & & & & $\mathrm{X}$ & & $\begin{array}{l}\$ 64 \text { million on cost } \\
\text { increase for caustic } \\
\text { dilution of feed and } \\
\text { additional saltstone } \\
\text { production }(820 \\
\text { Kgal./yr. saltstone } \\
\text { at } \$ 4 / \text { gal. and } 125 \\
\text { Kgal/yr. } 50 \% \text { wt } \\
\text { caustic at } \$ 4.25 / \text { gal } \\
\text { and two vaults at } \$ 9 \\
\text { million per vault. } \\
\text { HLW-SDT-99- } \\
0303\end{array}$ \\
\hline
\end{tabular}




\subsubsection{Direct Disposal in Grout}

\begin{tabular}{|c|c|c|c|c|c|c|c|c|c|c|c|c|c|}
\hline \multirow{2}{*}{$\begin{array}{l}\text { ID } \\
\text { No }\end{array}$} & \multirow{2}{*}{$\begin{array}{c}\text { Direct Disposal in } \\
\text { Grout } \\
\text { Uncertainty } \\
\text { Statement }\end{array}$} & \multicolumn{9}{|c|}{$\begin{array}{c}\text { Areas of } \\
\text { Uncertainty }\end{array}$} & \multirow[b]{2}{*}{1} & \multirow[b]{2}{*}{2} & \multirow[b]{2}{*}{ Explanatory Notes } \\
\hline & & $\begin{array}{l}\text { Mission } \\
\end{array}$ & $\begin{array}{l}\text { Technical } \\
\text { Maturity }\end{array}$ & Environmental & \begin{tabular}{|c|} 
Engincering \\
Design
\end{tabular} & $\begin{array}{c}\text { Operation } \\
\text { nat }\end{array}$ & Regulatory & Stakeholder & Safety & Radiological & & & \\
\hline 1 & $\begin{array}{l}\text { Existing vault } \\
\text { design may have to } \\
\text { be upgraded with } \\
\text { liners, ventilation } \\
\text { upgrades, } \\
\text { temperature } \\
\text { monitoring, } \\
\text { leachate collection, } \\
\text { capping/backfilling } \\
\text {, elimination of } \\
\text { floor penetrations, } \\
\text { HEPA filtration of } \\
\text { moist atmosphere } \\
\text { and the addition of } \\
\text { cell access for } \\
\text { failed equipment } \\
\text { disposal. }\end{array}$ & & & & $x$ & & & & & & $\mathrm{X}$ & & $\begin{array}{l}\$ 5 \text { million cost } \\
\text { increase for long } \\
\text { term hydrogen } \\
\text { collection system. } \\
\text { No Change. }\end{array}$ \\
\hline
\end{tabular}




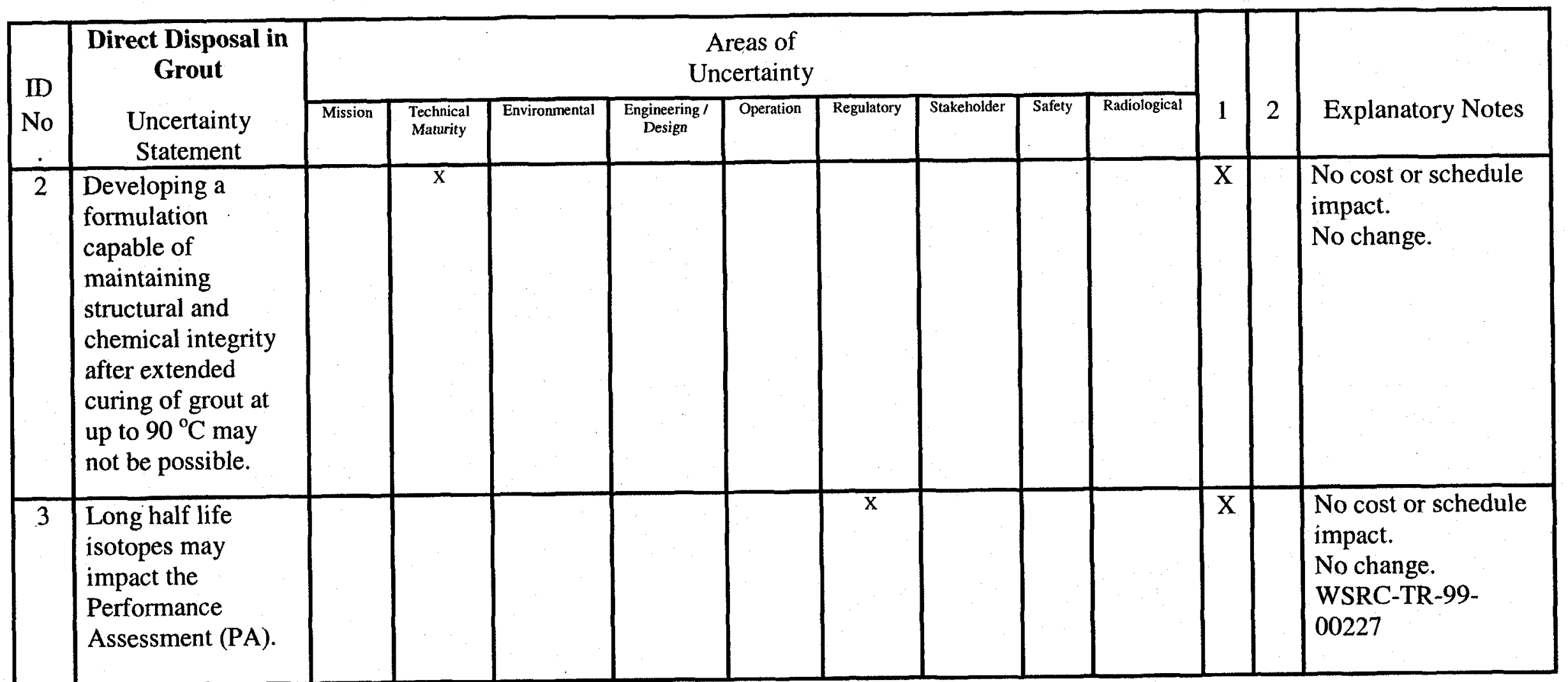




\begin{tabular}{|c|c|c|c|c|c|c|c|c|c|c|c|c|c|}
\hline \multirow{2}{*}{$\begin{array}{l}\text { ID } \\
\text { No }\end{array}$} & \multirow{2}{*}{$\begin{array}{c}\text { Direct Disposal in } \\
\text { Grout } \\
\text { Uncertainty } \\
\text { Statement } \\
\end{array}$} & \multicolumn{9}{|c|}{$\begin{array}{c}\text { Areas of } \\
\text { Uncertainty }\end{array}$} & \multirow[b]{2}{*}{1} & \multirow[b]{2}{*}{2} & \multirow[b]{2}{*}{ Explanatory Notes } \\
\hline & & Mission & $\begin{array}{l}\text { Technical } \\
\text { Maturity }\end{array}$ & Environmental & $\begin{array}{c}\text { Engineering/ } \\
\text { Design }\end{array}$ & Operation & Regulatory & Stakeholder & $\begin{array}{l}\text { Safety } \\
\end{array}$ & Radiological & & & \\
\hline 4 & $\begin{array}{l}\text { Difficulty in } \\
\text { filtration of sludge } \\
\text { and/or MST will } \\
\text { produce low filtrate } \\
\text { flow rates and } \\
\text { require frequent } \\
\text { cleaning }\end{array}$ & & & & $\mathrm{x}$ & & & & & & $\mathrm{X}$ & & $\begin{array}{l}\$ 6 \text { million cost } \\
\text { increase for the } \\
\text { larger filters. } \\
\$ 10 \text { million cost } \\
\text { increase for the } \\
\text { larger pumps. Flux } \\
\text { rate decrease from } \\
0.07 \text { to } 0.02 \text {. } \\
\text { WSRC-TR-99- } \\
00342\end{array}$ \\
\hline 5 & $\begin{array}{l}\text { TRU } \\
\text { decontamination } \\
\text { with MST is not } \\
\text { adequate with the } \\
\text { design residence } \\
\text { time. }\end{array}$ & & $\mathrm{x}$ & & & & & & & & $\mathrm{X}$ & & $\begin{array}{l}125 \mathrm{Kgal} \text {. double } \\
\text { lobe tank for dilution } \\
\text { effect. MST } \\
\text { concentration and } \\
\text { residence time } \\
\text { requires more } \\
\text { research for } \\
\text { bounding waste. No } \\
\text { additional cost } \\
\text { beyond Item } 28 \text {. }\end{array}$ \\
\hline 6 & $\begin{array}{l}\text { MST amount } \\
\text { needed for } \\
\text { decontamination } \\
\text { exceeds DWPF Ti } \\
\text { glass limits. }\end{array}$ & & & & & & $\mathrm{x}$ & & & & & $X$ & $\begin{array}{l}\text { MST concentration } \\
\text { of } 0.4 \mathrm{~g} / \mathrm{L} \text { makes } \\
\text { acceptable glass. } \\
\text { WSRC-TR-99- } \\
00332\end{array}$ \\
\hline
\end{tabular}




\begin{tabular}{|c|c|c|c|c|c|c|c|c|c|c|c|c|c|}
\hline \multirow{2}{*}{$\begin{array}{l}\text { ID } \\
\text { No }\end{array}$} & \multirow{2}{*}{$\begin{array}{c}\text { Direct Disposal in } \\
\text { Grout } \\
\text { Uncertainty } \\
\text { Statement }\end{array}$} & \multicolumn{9}{|c|}{$\begin{array}{c}\text { Areas of } \\
\text { Uncertainty }\end{array}$} & \multirow[b]{2}{*}{1} & \multirow[b]{2}{*}{2} & \multirow[b]{2}{*}{ Explanatory Notes } \\
\hline & & Mission & $\begin{array}{l}\text { Technical } \\
\text { Maturity }\end{array}$ & Environmental & $\begin{array}{c}\text { Engineering } / \\
\text { Design }\end{array}$ & Operation & Regulatory & Stakehoider & Safety & Radiological & & & \\
\hline 7 & $\begin{array}{l}\text { Analysis delay (1 } \\
\text { week) in measuring } \\
\text { for Sr DF in MST } \\
\text { process. }\end{array}$ & & $\bar{X}$ & & & & & & & & $\mathrm{X}$ & & $\begin{array}{l}\text { No cost or schedule } \\
\text { impact, within } \\
\text { existing R\&D scope } \\
\text { and schedule } \\
\text { duration. } \\
\text { No change. }\end{array}$ \\
\hline 8 & $\begin{array}{l}\text { What is the fate of } \\
\text { Am in the process? }\end{array}$ & & $\bar{x}$ & & & & & & & & & $X$ & $\begin{array}{l}\text { N/A } \\
\text { No change. }\end{array}$ \\
\hline 9 & $\begin{array}{l}\text { Process not } \\
\text { acceptable to } \\
\text { general public. }\end{array}$ & & & & & & & $\bar{x}$ & & & $\bar{X}$ & & $\begin{array}{l}24 \text { month delay in } \\
\text { start of final design. } \\
\text { Can start at end of } \\
\text { conceptual design } \\
\text { based on NEPA } \\
\text { documentation. } \\
\text { No change. } \\
\text { October } 1998 \\
\text { Stakeholder Focus } \\
\text { Group of CAB. }\end{array}$ \\
\hline 10 & $\begin{array}{l}\text { Potential for } \\
\text { extended delay } \\
\text { from NEPA/EIS } \\
\text { process. }\end{array}$ & & & & & & $\bar{X}$ & & & & $\bar{X}$ & & $\begin{array}{l}\text { No impact beyond } \\
\text { Item } 9 . \\
\text { No change. }\end{array}$ \\
\hline
\end{tabular}




\begin{tabular}{|c|c|c|c|c|c|c|c|c|c|c|c|c|c|}
\hline \multirow{2}{*}{$\begin{array}{l}\text { ID } \\
\text { No }\end{array}$} & \multirow{2}{*}{$\begin{array}{c}\text { Direct Disposal in } \\
\text { Grout } \\
\text { Uncertainty } \\
\text { Statement }\end{array}$} & \multicolumn{9}{|c|}{$\begin{array}{c}\text { Areas of } \\
\text { Uncertainty }\end{array}$} & \multirow[b]{2}{*}{1} & \multirow[b]{2}{*}{2} & \multirow[b]{2}{*}{ Explanatory Notes } \\
\hline & & Mission & $\begin{array}{l}\text { Technical } \\
\text { Maturity }\end{array}$ & Environmental & $\begin{array}{c}\text { Engineering / } \\
\text { Design }\end{array}$ & Operation & Regulatory & Stakeholder & Safety & $\begin{array}{l}\text { Radiological } \\
\end{array}$ & & & \\
\hline 11 & $\begin{array}{l}\text { Technical } \\
\text { regulatory agencies } \\
\text { may delay } . \\
\text { approvals. }\end{array}$ & & & & & & $\mathrm{X}$ & & & & $X$ & & $\begin{array}{l}5 \text { year delay to } \\
\text { complete } \\
\text { construction for high } \\
\text { level waste in SC. } 2 \\
\text { year delay in } \\
\text { radioactive operation } \\
\text { for redesign and } \\
\text { EIS. } \\
\text { No change. }\end{array}$ \\
\hline 12 & $\begin{array}{l}\text { Political } \\
\text { representatives of } \\
\text { public may delay } \\
\text { approvals. }\end{array}$ & & & & & & & $\mathrm{x}$ & & & $\mathrm{X}$ & & $\begin{array}{l}\text { No additional cost or } \\
\text { schedule impact } \\
\text { beyond Item } 11 . \\
\text { No change. }\end{array}$ \\
\hline 13 & $\begin{array}{l}\text { Process not } \\
\text { technically } \\
\text { supportive of future } \\
\text { missions (e.g. can- } \\
\text { in-can) }\end{array}$ & $x$ & & & & & & & & & $\mathrm{X}$ & & $\begin{array}{l}\$ 50 \text { million cost } \\
\text { increase to support } \\
\text { commitment to can- } \\
\text { in-can mission. } \\
\text { No change. }\end{array}$ \\
\hline
\end{tabular}




\begin{tabular}{|c|c|c|c|c|c|c|c|c|c|c|c|c|c|}
\hline \multirow{2}{*}{$\begin{array}{l}\text { ID } \\
\text { No }\end{array}$} & \multirow{2}{*}{$\begin{array}{c}\text { Direct Disposal in } \\
\text { Grout } \\
\text { Uncertainty } \\
\text { Statement }\end{array}$} & \multicolumn{9}{|c|}{$\begin{array}{c}\text { Areas of } \\
\text { Uncertainty }\end{array}$} & \multirow[b]{2}{*}{1} & \multirow[b]{2}{*}{2} & \multirow[b]{2}{*}{ Explanatory Notes } \\
\hline & & Mission & $\begin{array}{l}\text { Technical } \\
\text { Maturity }\end{array}$ & Environmental & $\begin{array}{c}\text { Engineering/ } \\
\text { Design }\end{array}$ & Operation & Regulatory & Stakeholder & Safety & Radiological & & & \\
\hline 14 & $\begin{array}{l}\text { DOE independent } \\
\text { project review and } \\
\text { acceptance may } \\
\text { impact project } \\
\text { milestones. }\end{array}$ & & & & & & $\mathrm{X}$ & & & & $\mathrm{X}$ & & $\begin{array}{l}\text { Schedule impact of } 1 \\
\text { month at end of } \\
\text { conceptual design, } 1 \\
\text { month at the end of } \\
\text { preliminary design, } \\
2 \text { months at the end } \\
\text { of final design and } \\
12 \text { months prior to } \\
\text { radioactive } \\
\text { operations. } \\
\text { No change. }\end{array}$ \\
\hline 15 & $\begin{array}{l}\text { Change in } \\
\text { requirements and } \\
\text { standards, such as } \\
\text { NRC licensing may } \\
\text { impact the cost and } \\
\text { schedule. }\end{array}$ & & & & & & $\mathrm{x}$ & & & & $\bar{X}$ & & $\begin{array}{l}\text { No additional cost or } \\
\text { schedule impact } \\
\text { beyond Item } 11 . \\
\text { NRC standards } \\
\text { equivalency will be } \\
\text { part of the design } \\
\text { process. } \\
\text { No change. } \\
\text { HLW-SDT-99-0062 }\end{array}$ \\
\hline
\end{tabular}




\begin{tabular}{|c|c|c|c|c|c|c|c|c|c|c|c|c|c|}
\hline \multirow{2}{*}{$\begin{array}{l}\text { ID } \\
\text { No }\end{array}$} & \multirow{2}{*}{$\begin{array}{c}\text { Direct Disposal in } \\
\text { Grout } \\
\text { Uncertainty } \\
\text { Statement }\end{array}$} & \multicolumn{9}{|c|}{$\begin{array}{c}\text { Areas of } \\
\text { Uncertainty }\end{array}$} & \multirow[b]{2}{*}{1} & \multirow[b]{2}{*}{2} & \multirow[b]{2}{*}{ Explanatory Notes } \\
\hline & & $\begin{array}{c}\text { Mission } \\
\end{array}$ & $\begin{array}{l}\text { Technical } \\
\text { Marurity }\end{array}$ & $\begin{array}{c}\text { Environmental } \\
\end{array}$ & $\begin{array}{c}\text { Engineering/ } \\
\text { Design }\end{array}$ & $\begin{array}{l}\text { Operation } \\
\end{array}$ & $\begin{array}{l}\text { Regulatoryy } \\
\text { Res }\end{array}$ & Stakeholder & $\begin{array}{c}\text { Safety } \\
\end{array}$ & $\begin{array}{l}\text { Radiological } \\
\end{array}$ & & & \\
\hline 16 & $\begin{array}{l}\text { The interfacing } \\
\text { facilities } \\
\text { operational } \\
\text { schedules may } \\
\text { impact completion } \\
\text { of tie-ins to the new } \\
\text { facility. }\end{array}$ & & & & & $x$ & & & & & $X$ & & $\begin{array}{l}\text { No additional cost or } \\
\text { schedule impact } \\
\text { based on opportunity } \\
\text { to coordinate with } \\
\text { DWPF outages. } \\
\text { No change. }\end{array}$ \\
\hline 17 & $\begin{array}{l}\text { DOE lack of } \\
\text { support of required } \\
\text { budget and } \\
\text { schedule may delay } \\
\text { new facility startup. }\end{array}$ & & & & & & & $\mathrm{x}$ & & & $\mathrm{X}$ & & $\begin{array}{l}3 \text { month schedule } \\
\text { impact in the first } \\
\text { year. } \\
5 \text { month schedule } \\
\text { impact in the second } \\
\text { year. } \\
\text { No change. }\end{array}$ \\
\hline 18 & $\begin{array}{l}\text { SRS infrastructure } \\
\text { may not support the } \\
\text { project needs. }\end{array}$ & & & & & $\bar{x}$ & & & & & $\mathrm{X}$ & & $\begin{array}{l}\$ 20 \text { million cost } \\
\text { increase for } \\
\text { overtime resulting } \\
\text { from staffing delays. } \\
\text { No change. }\end{array}$ \\
\hline
\end{tabular}




\begin{tabular}{|c|c|c|c|c|c|c|c|c|c|c|c|c|c|}
\hline \multirow{2}{*}{$\begin{array}{l}\text { ID } \\
\text { No }\end{array}$} & \multirow{2}{*}{$\begin{array}{c}\text { Direct Disposal in } \\
\text { Grout } \\
\text { Uncertainty } \\
\text { Statement } \\
\end{array}$} & \multicolumn{9}{|c|}{$\begin{array}{c}\text { Areas of } \\
\text { Uncertainty }\end{array}$} & \multirow[b]{2}{*}{1} & \multirow[b]{2}{*}{2} & \multirow[b]{2}{*}{ Explanatory Notes } \\
\hline & & Mission & $\begin{array}{l}\text { Technical } \\
\text { Maturity }\end{array}$ & Environmental & $\begin{array}{c}\text { Enginecring' } \\
\text { Design }\end{array}$ & Operation & Regulatory & Stakeholder & Safety & Radiological & & & \\
\hline 19 & $\begin{array}{l}\text { Waste removal is } \\
\text { being accelerated. } \\
\text { May conflict with } \\
\text { preferred } \\
\text { alternative or } \\
\text { preferred } \\
\text { alternative may } \\
\text { divert resources } \\
\text { from waste removal } \\
\text { project. }\end{array}$ & $\bar{x}$ & & & & & & & & & $X$ & & $\begin{array}{l}\text { No additional cost or } \\
\text { schedule impact } \\
\text { beyond Item } 17 . \\
\text { No change. }\end{array}$ \\
\hline
\end{tabular}




\begin{tabular}{|c|c|c|c|c|c|c|c|c|c|c|c|c|c|}
\hline \multirow{2}{*}{$\begin{array}{l}\text { ID } \\
\text { No }\end{array}$} & \multirow{2}{*}{$\begin{array}{c}\text { Direct Disposal in } \\
\text { Grout } \\
\text { Uncertainty } \\
\text { Statement }\end{array}$} & \multicolumn{9}{|c|}{$\begin{array}{c}\text { Areas of } \\
\text { Uncertainty }\end{array}$} & \multirow[b]{2}{*}{1} & \multirow[b]{2}{*}{2} & \multirow[b]{2}{*}{ Explanatory Notes } \\
\hline & & Mission & $\begin{array}{l}\text { Technical } \\
\text { Maturity }\end{array}$ & Environmental & $\begin{array}{c}\text { Engineering / } \\
\text { Design }\end{array}$ & Operation & Regulatory & Stakeholder & Safety & Radiological & & & \\
\hline 20 & $\begin{array}{l}\text { Pressure on 'old' } \\
\text { infrastructure will } \\
\text { increase, } \\
\text { endangering } \\
\text { schedule due to } \\
\text { three fold increase } \\
\text { in flow } \\
\text { requirements from } \\
\text { HTF and FTF. This } \\
\text { would endanger } \\
\text { performance of } \\
\text { infrastructure. }\end{array}$ & & & & & $\bar{x}$ & & & & & $\mathrm{X}$ & & $\begin{array}{l}9 \text { month delay in } \\
\text { completing salt } \\
\text { removal from a } \\
\text { production schedule } \\
\text { delay to reach salt } \\
\text { solution feed rate } \\
\text { assumption. Basis: } \\
50 \% \text { material } \\
\text { movement in the } \\
\text { first year results in } 6 \\
\text { months and } 75 \% \\
\text { material movement } \\
\text { in the second year } \\
\text { results in } 3 \text { months. } \\
\text { No change. }\end{array}$ \\
\hline 21 & $\begin{array}{l}\text { A clearly defined } \\
\text { safety strategy } \\
\text { should be agreed to } \\
\text { by the end of } \\
\text { conceptual design } \\
\text { to preclude } \\
\text { schedule impacts. }\end{array}$ & & & & & & & & $\bar{x}$ & & $\mathrm{X}$ & & $\begin{array}{l}\text { No additional cost or } \\
\text { schedule impact } \\
\text { beyond Item } 11 . \\
\text { No change. }\end{array}$ \\
\hline
\end{tabular}




\begin{tabular}{|c|c|c|c|c|c|c|c|c|c|c|c|c|c|}
\hline \multirow{2}{*}{$\begin{array}{l}\text { ID } \\
\text { No }\end{array}$} & \multirow{2}{*}{$\begin{array}{c}\text { Direct Disposal in } \\
\text { Grout } \\
\text { Uncertainty } \\
\text { Statement }\end{array}$} & \multicolumn{9}{|c|}{$\begin{array}{c}\text { Areas of } \\
\text { Uncertainty }\end{array}$} & \multirow[b]{2}{*}{1} & \multirow[b]{2}{*}{2} & \multirow[b]{2}{*}{ Explanatory Notes } \\
\hline & & Mission & $\begin{array}{l}\text { Technical } \\
\text { Maturity }\end{array}$ & Environmental & $\begin{array}{c}\text { Engineering/ } \\
\text { Design }\end{array}$ & Operation & $\overline{\text { Regulatory }}$ & Stakeholder & Safety & Radiological & & & \\
\hline 22 & $\begin{array}{l}\text { Improper contract } \\
\text { strategy for design } \\
\text { work may impact } \\
\text { the schedule. }\end{array}$ & & & & $\bar{x}$ & & & & & & $\bar{X}$ & & $\begin{array}{l}6 \text { month delay in } \\
\text { completing } \\
\text { conceptual design. } \\
\text { No change. }\end{array}$ \\
\hline 23 & $\begin{array}{l}\text { Dry material } \\
\text { handling may be a } \\
\text { problem. }\end{array}$ & & & & $\bar{x}$ & & & & & & & $\mathrm{X}$ & $\begin{array}{l}\text { N/A } \\
\text { No change. }\end{array}$ \\
\hline 24 & $\begin{array}{l}\text { Geotechnical } \\
\text { problems with } \\
\text { siting locations } \\
\text { may cause schedule } \\
\text { delays. }\end{array}$ & & & & $\bar{x}$ & & & & & & $\mathrm{X}$ & & $\begin{array}{l}12 \text { month delay in } \\
\text { start of final design. } \\
\$ 105 \text { million cost } \\
\text { increase (based on } \\
10 \% \text { of TEC }+\$ 34 \\
\text { million for } \\
\text { substructure grout }+ \\
\text { contingency } \\
\text { percentage). } \\
\text { No change. }\end{array}$ \\
\hline 25 & $\begin{array}{l}\text { GT-73 unit } \\
\text { operations may not } \\
\text { be required. }\end{array}$ & & & & $\mathrm{x}$ & & & & & & $\mathrm{X}$ & & $\begin{array}{l}\$ 27 \text { million cost } \\
\text { decrease. } \\
\text { No change. } \\
\text { WSRC-RP-99- } \\
00006\end{array}$ \\
\hline
\end{tabular}




\begin{tabular}{|c|c|c|c|c|c|c|c|c|c|c|c|c|c|}
\hline \multirow{2}{*}{$\begin{array}{l}\text { ID } \\
\text { No }\end{array}$} & \multirow{2}{*}{$\begin{array}{c}\text { Direct Disposal in } \\
\text { Grout } \\
\text { Uncertainty } \\
\text { Statement } \\
\end{array}$} & \multicolumn{9}{|c|}{$\begin{array}{c}\text { Areas of } \\
\text { Uncertainty }\end{array}$} & \multirow[b]{2}{*}{1} & \multirow[b]{2}{*}{2} & \multirow[b]{2}{*}{ Explanatory Notes } \\
\hline & & Mission & $\begin{array}{l}\text { Technical } \\
\text { Maturity }\end{array}$ & Environmental & $\begin{array}{c}\text { Engineering / } \\
\text { Design }\end{array}$ & Operation & Regulatory & Stakeholder & Safety & $\begin{array}{l}\text { Radiological } \\
\end{array}$ & & & \\
\hline 26 & $\begin{array}{l}\text { DWPF recycle } \\
\text { stream does not } \\
\text { contain cesium } \\
\text { concentration } \\
\text { assumed in HLW } \\
\text { System Plan. }\end{array}$ & & & & & $\mathrm{x}$ & & & & & $\mathrm{X}$ & & $\begin{array}{l}\$ 65 \text { million cost } \\
\text { decrease. Basis is } \\
\text { DWPF recycle } \\
\text { rerouted to ETF } \\
\text { saving evaporator } \\
\text { operation. } \\
\text { No change. }\end{array}$ \\
\hline 27 & $\begin{array}{l}\text { Suspect product } \\
\text { may not be able to } \\
\text { be recovered. }\end{array}$ & & & & & $\mathrm{X}$ & & & & & $\mathrm{X}$ & & $\begin{array}{l}\$ 9 \text { million cost } \\
\text { increase based on } \\
\text { abandoning a vault. } \\
\text { No change. }\end{array}$ \\
\hline 28 & $\begin{array}{l}\text { Difficulty in } \\
\text { resuspending MST } \\
\text { after long quiescent } \\
\text { period will require } \\
\text { temperature control } \\
\text { and mixing } \\
\text { equipment. }\end{array}$ & & & & $\mathrm{x}$ & & & & & & $\mathrm{x}$ & & $\begin{array}{l}\$ 10 \text { million cost } \\
\text { increase (based on } \\
\text { the cost estimate for } \\
\text { a } 100 \text { Kgal. Alpha } \\
\text { adsorption tank). } \\
\text { ORNLTM- } \\
\text { 1999/166 }\end{array}$ \\
\hline
\end{tabular}




\subsubsection{Small Tank TPB Precipitation}

\begin{tabular}{|c|c|c|c|c|c|c|c|c|c|c|c|c|c|}
\hline \multirow{2}{*}{$\begin{array}{l}\text { ID } \\
\text { No }\end{array}$} & \multirow{2}{*}{$\begin{array}{c}\text { Small Tank TPB } \\
\text { Precipitation } \\
\text { Uncertainty } \\
\text { Statement }\end{array}$} & \multicolumn{9}{|c|}{$\begin{array}{l}\text { Areas of } \\
\text { Uncertainty }\end{array}$} & \multirow[b]{2}{*}{1} & \multirow[b]{2}{*}{2} & \multirow[b]{2}{*}{$\begin{array}{l}\text { Explanatory } \\
\text { Notes }\end{array}$} \\
\hline & & $\begin{array}{l}\text { Mission } \\
\end{array}$ & $\begin{array}{l}\text { Technical } \\
\text { Maunityty }\end{array}$ & Environmental & $\begin{array}{c}\text { Engineering! } \\
\text { Design }\end{array}$ & Operation & $\begin{array}{l}\text { Regulatory } \\
\end{array}$ & Stakeholder & $\begin{array}{l}\text { Safety } \\
\text { s. }\end{array}$ & $\overline{\text { Radiological }}$ & & & \\
\hline 1 & $\begin{array}{l}\text { Close coupled unit } \\
\text { operations adds } \\
\text { production } \\
\text { complexity. Salt } \\
\text { Cell in DWPF has to } \\
\text { be operated in this } \\
\text { option. }\end{array}$ & & & & & $\bar{x}$ & & & & & $\mathrm{X}$ & & $\begin{array}{l}\$ 80 \text { million } \\
\text { increase to } \\
\text { relocate } \\
\text { precipitate } \\
\text { hydrolysis } \\
\text { process from } \\
\text { DWPF to the new } \\
\text { Salt Disposition } \\
\text { Facility and } \\
\text { increase } \\
\text { equipment sizing } \\
\text { to achieve tank } \\
\text { farm waste } \\
\text { handling } \\
\text { limitation. 28 } \\
\text { months operating } \\
\text { time reduction } \\
\text { due to increased } \\
\text { process rate. } \\
\text { HLW-SDT-99- } \\
\text { 0266 } \\
\text { WSRC-RP-99- } \\
\text { 00006 } \\
\text { WSRC-RP-99- } \\
\text { 00005 }\end{array}$ \\
\hline
\end{tabular}


Page 182 of 277

\begin{tabular}{|c|c|c|c|c|c|c|c|c|c|c|c|c|c|}
\hline \multirow{2}{*}{$\begin{array}{l}\text { ID } \\
\text { No }\end{array}$} & \multirow{2}{*}{$\begin{array}{c}\text { Small Tank TPB } \\
\text { Precipitation } \\
\text { Uncertainty } \\
\text { Statement }\end{array}$} & \multicolumn{9}{|c|}{$\begin{array}{c}\text { Areas of } \\
\text { Uncertainty }\end{array}$} & \multirow[b]{2}{*}{1} & \multirow[b]{2}{*}{2} & \multirow[b]{2}{*}{$\begin{array}{c}\text { Explanatory } \\
\text { Notes }\end{array}$} \\
\hline & & Mission & $\begin{array}{l}\text { Technical } \\
\text { Maturity }\end{array}$ & Environmental & $\begin{array}{c}\text { Engineering/ } \\
\text { Design }\end{array}$ & Operation & Regulatory & Stakeholder & Safety & $\overline{\text { Radiological }}$ & & & \\
\hline 2 & $\begin{array}{l}\text { Benzene releases } \\
\text { may exceed permit } \\
\text { levels due to } \\
\text { additional } \\
\text { (unknown) catalytic } \\
\text { effects or catalyst } \\
\text { build-up through } \\
\text { plate-out. }\end{array}$ & & $\mathrm{x}$ & & & & & & & & $X$ & & $\begin{array}{l}\text { Benzene emission } \\
\text { reduction system } \\
\text { estimated at } \$ 5 \\
\text { million to meet } \\
\text { permit limits. } \\
\text { No change. }\end{array}$ \\
\hline 3 & $\begin{array}{l}\text { Limited experience } \\
\text { with the hydrolysis } \\
\text { of fresh precipitate } \\
\text { in the Salt Process } \\
\text { Cell. }\end{array}$ & & $\mathrm{x}$ & & & & & & & & & $\mathrm{X}$ & $\begin{array}{l}\text { N/A } \\
\text { No change. } \\
\text { WSRC-TR-99- } \\
00272\end{array}$ \\
\hline 4 & $\begin{array}{l}\text { Process will not } \\
\text { produce the DF } \\
\text { required because of } \\
\text { slow kinetics of } \\
\text { MST and TPB. }\end{array}$ & & & & $\mathrm{x}$ & & & & & & & $\mathrm{X}$ & $\begin{array}{l}\text { R\&D results } \\
\text { indicate the } \\
\text { design basis is } \\
\text { acceptable. } \\
\text { WSRC-TR-99- } \\
\text { 00345 } \\
\text { ORNL/TM- } \\
1999 / 234\end{array}$ \\
\hline
\end{tabular}




\begin{tabular}{|c|c|c|c|c|c|c|c|c|c|c|c|c|c|}
\hline \multirow{2}{*}{$\begin{array}{l}\text { ID } \\
\text { No }\end{array}$} & \multirow{2}{*}{$\begin{array}{c}\text { Small Tank TPB } \\
\text { Precipitation } \\
\text { Uncertainty } \\
\text { Statement }\end{array}$} & \multicolumn{9}{|c|}{$\begin{array}{c}\text { Areas of } \\
\text { Uncertainty }\end{array}$} & \multirow[b]{2}{*}{1} & \multirow[b]{2}{*}{2} & \multirow[b]{2}{*}{$\begin{array}{c}\text { Explanatory } \\
\text { Notes }\end{array}$} \\
\hline & & Mission & $\begin{array}{l}\text { Technical } \\
\text { Maturity }\end{array}$ & Environmental & $\begin{array}{c}\text { Engineering/ } \\
\text { Design }\end{array}$ & Operation & Regulatory & Stakeholder & $\begin{array}{l}\text { Safety } \\
\end{array}$ & Radiological & & & \\
\hline 5 & $\begin{array}{l}\text { Process chemistry } \\
\text { understanding and } \\
\text { application are being } \\
\text { verified. ( } 96-1 \\
\text { Lessons Learned) }\end{array}$ & & $\bar{x}$ & & & & & & & & $\mathrm{X}$ & & $\begin{array}{l}4 \text { month schedule } \\
\text { delay in } \\
\text { completing } \\
\text { preliminary } \\
\text { design to resolve } \\
\text { foaming issues in } \\
\text { the process } \\
\text { vessels.Catalyst } \\
\text { activation greater } \\
\text { than the bounding } \\
\text { case results in a } \\
\text { loss of product } \\
\text { DF requiring } \\
\text { operational delays } \\
\text { for resolution. } 6 \\
\text { month delay in } \\
\text { completing } \\
\text { operation due to } \\
\text { loss of one macro } \\
\text { batch every } 4 \\
\text { years and } 2 \\
\text { months to recover } \\
\text { a macro batch. } \\
\text { WSRC-TR-99- } \\
\text { 00279 } \\
\text { WSRC-TR-99- } \\
00345\end{array}$ \\
\hline
\end{tabular}




\begin{tabular}{|c|c|c|c|c|c|c|c|c|c|c|c|c|c|}
\hline \multirow{2}{*}{$\begin{array}{l}\text { ID } \\
\text { No }\end{array}$} & \multirow{2}{*}{$\begin{array}{c}\text { Small Tank TPB } \\
\text { Precipitation } \\
\text { Uncertainty } \\
\text { Statement } \\
\end{array}$} & \multicolumn{9}{|c|}{$\begin{array}{c}\text { Areas of } \\
\text { Uncertainty }\end{array}$} & \multirow[b]{2}{*}{1} & \multirow[b]{2}{*}{2} & \multirow[b]{2}{*}{$\begin{array}{l}\text { Explanatory } \\
\text { Notes }\end{array}$} \\
\hline & & Mission & $\begin{array}{l}\text { Technical } \\
\text { Maturity }\end{array}$ & Environmental & $\begin{array}{c}\text { Engineering / } \\
\text { Design }\end{array}$ & Operation & Regulatory & Stakeholder & Safety & Radiological & & & \\
\hline 6 & $\begin{array}{l}\text { MST amount needed } \\
\text { for decontamination } \\
\text { exceeds DWPF Ti } \\
\text { glass limits. }\end{array}$ & & & & & & $\mathrm{X}$ & & & & & $\mathrm{X}$ & $\begin{array}{l}\text { MST } \\
\text { concentration of } \\
0.4 \text { g/L makes } \\
\text { acceptable glass. } \\
\text { WSRC-TR-99- } \\
00332 \\
\end{array}$ \\
\hline 7 & $\begin{array}{l}\text { Analysis delay (1 } \\
\text { week) in measuring } \\
\text { for Sr DF in MST } \\
\text { process. }\end{array}$ & & $\mathrm{x}$ & & & & & & & & $X$ & & $\begin{array}{l}\text { No cost or } \\
\text { schedule impact, } \\
\text { within existing } \\
\text { R\&D scope and } \\
\text { schedule duration. } \\
\text { No change. }\end{array}$ \\
\hline 8 & $\begin{array}{l}\text { What is the fate of } \\
\text { Am in the process? }\end{array}$ & & $\bar{X}$ & & & & & & & & & $X$ & $\begin{array}{l}\text { N/A } \\
\text { No change. } \\
\text { October } 1998 \\
\text { Stakeholder } \\
\text { Focus Group of } \\
\text { CAB. }\end{array}$ \\
\hline 9 & $\begin{array}{l}\text { Stakeholders will } \\
\text { reject the alternative. } \\
\text { Similar to large tank. }\end{array}$ & & & & & & & $\mathrm{x}$ & & & $X$ & & $\begin{array}{l}\text { Covered within } \\
\text { the existing cost } \\
\text { and schedule } \\
\text { estimate. } \\
\text { No change. } \\
\text { October } 1998 \\
\text { Stakeholder } \\
\text { Focus Group of } \\
\text { CAB. }\end{array}$ \\
\hline
\end{tabular}




\begin{tabular}{|c|c|c|c|c|c|c|c|c|c|c|c|c|c|}
\hline \multirow{2}{*}{$\begin{array}{l}\text { ID } \\
\text { No }\end{array}$} & \multirow{2}{*}{$\begin{array}{c}\text { Small Tank TPB } \\
\text { Precipitation } \\
\text { Uncertainty } \\
\text { Statement }\end{array}$} & \multicolumn{9}{|c|}{$\begin{array}{c}\text { Areas of } \\
\text { Uncertainty }\end{array}$} & \multirow[b]{2}{*}{1} & \multirow[b]{2}{*}{2} & \multirow[b]{2}{*}{$\begin{array}{c}\text { Explanatory } \\
\text { Notes }\end{array}$} \\
\hline & & Mission & $\begin{array}{l}\text { Technical } \\
\text { Maturity }\end{array}$ & Environmental & $\begin{array}{c}\text { Engineering/ } \\
\text { Design }\end{array}$ & Operation & Regulatory & $\begin{array}{l}\text { Stakeholder } \\
\text { Stakehold }\end{array}$ & Safety & Radiological & & & \\
\hline 10 & $\begin{array}{l}\text { Geotechnical } \\
\text { problems with siting } \\
\text { locations may cause } \\
\text { schedule delays. }\end{array}$ & & & & $\mathrm{x}$ & & & & & & & $X$ & $\begin{array}{l}\text { Site selection and } \\
\text { geotechnical } \\
\text { characterization } \\
\text { was completed } \\
\text { with no } \\
\text { subsurface } \\
\text { concerns. } \\
\text { WSRC-RP-99- } \\
00513\end{array}$ \\
\hline 11 & $\begin{array}{l}\text { Organics fed to tank } \\
\text { farms. }\end{array}$ & $\mathrm{x}$ & & & & & & & & & & $\mathrm{X}$ & $\begin{array}{l}\text { N/A } \\
\text { No change. }\end{array}$ \\
\hline
\end{tabular}




\begin{tabular}{|c|c|c|c|c|c|c|c|c|c|c|c|c|c|}
\hline \multirow{2}{*}{$\begin{array}{l}\text { ID } \\
\text { No }\end{array}$} & \multirow{2}{*}{$\begin{array}{c}\text { Small Tank TPB } \\
\text { Precipitation } \\
\text { Uncertainty } \\
\text { Statement }\end{array}$} & \multicolumn{9}{|c|}{$\begin{array}{c}\text { Areas of } \\
\text { Uncertainty }\end{array}$} & \multirow[b]{2}{*}{1} & \multirow[b]{2}{*}{2} & \multirow[b]{2}{*}{$\begin{array}{c}\text { Explanatory } \\
\text { Notes }\end{array}$} \\
\hline & & Mission & $\begin{array}{l}\text { Technical } \\
\text { Maturity }\end{array}$ & Environmental & $\begin{array}{c}\text { Engineering / } \\
\text { Design }\end{array}$ & Operation & Regulatory & Stakeholder & Safety & $\begin{array}{l}\text { Radiological } \\
\text {. }\end{array}$ & & & \\
\hline 12 & $\begin{array}{l}\text { DOE independent } \\
\text { project review and } \\
\text { acceptance may } \\
\text { impact project } \\
\text { milestones. }\end{array}$ & & & & & & $\bar{x}$ & & & & $\mathrm{X}$ & & $\begin{array}{l}\text { Schedule impact } \\
\text { of } 3 \text { month to } \\
\text { start conceptual } \\
\text { design for } \\
\text { addressing GAO } \\
\text { issues, } 1 \text { month at } \\
\text { end of conceptual } \\
\text { design, } 1 \text { month } \\
\text { at the end of } \\
\text { preliminary } \\
\text { design, } 2 \text { months } \\
\text { at the end of final } \\
\text { design and } 1 \\
\text { month prior to } \\
\text { radioactive } \\
\text { operations. } \\
\text { GAO/RECD-99- } \\
69\end{array}$ \\
\hline
\end{tabular}




\begin{tabular}{|c|c|c|c|c|c|c|c|c|c|c|c|c|c|}
\hline \multirow{2}{*}{$\begin{array}{l}\text { ID } \\
\text { No }\end{array}$} & \multirow{2}{*}{$\begin{array}{c}\text { Small Tank TPB } \\
\text { Precipitation } \\
\text { Uncertainty } \\
\text { Statement }\end{array}$} & \multicolumn{9}{|c|}{$\begin{array}{c}\text { Areas of } \\
\text { Uncertainty }\end{array}$} & \multirow[b]{2}{*}{1} & \multirow[b]{2}{*}{2} & \multirow[b]{2}{*}{$\begin{array}{c}\text { Explanatory } \\
\text { Notes }\end{array}$} \\
\hline & & $\begin{array}{l}\text { Mission } \\
\end{array}$ & $\begin{array}{l}\text { Technical } \\
\text { Maturity }\end{array}$ & Environmental & $\begin{array}{c}\text { Engineering } \\
\text { Design }\end{array}$ & Operation & Regulatory & Stakeholder & Safety & Radiological & & & \\
\hline 13 & $\begin{array}{l}\text { Change in } \\
\text { requirements and } \\
\text { standards, such as } \\
\text { NRC licensing may } \\
\text { impact the cost and } \\
\text { schedule. }\end{array}$ & & & & & & $\mathrm{x}$ & & & & $X$ & & $\begin{array}{l}18 \text { month delay to } \\
\text { radioactive } \\
\text { operations. } \\
\text { Additional } \$ 1 \\
\text { million cost. SAR } \\
\text { may cause } 4 \\
\text { month delay in } \\
\text { completing } \\
\text { preliminary } \\
\text { design. NRC } \\
\text { standards } \\
\text { equivalency will } \\
\text { be part of design } \\
\text { process. } \\
\text { HLW-SDT-99- } \\
\text { 0062 } \\
\text { No change. } \\
\end{array}$ \\
\hline 14 & $\begin{array}{l}\text { The interfacing } \\
\text { facilities operational } \\
\text { schedules may } \\
\text { impact completion } \\
\text { of tie-ins to the new } \\
\text { facility. }\end{array}$ & & & & & $\mathrm{x}$ & & & & & $X$ & & $\begin{array}{l}2 \text { month } \\
\text { production delay } \\
\text { for DWPF to } \\
\text { install new } \\
\text { transfer line. } \\
\text { Based on number } \\
1 .\end{array}$ \\
\hline
\end{tabular}




\begin{tabular}{|c|c|c|c|c|c|c|c|c|c|c|c|c|c|}
\hline \multirow{2}{*}{$\begin{array}{l}\text { ID } \\
\text { No }\end{array}$} & \multirow{2}{*}{$\begin{array}{c}\text { Small Tank TPB } \\
\text { Precipitation } \\
\text { Uncertainty } \\
\text { Statement } \\
\end{array}$} & \multicolumn{9}{|c|}{$\begin{array}{c}\text { Areas of } \\
\text { Uncertainty }\end{array}$} & \multirow[b]{2}{*}{1} & \multirow[b]{2}{*}{2} & \multirow[b]{2}{*}{$\begin{array}{c}\text { Explanatory } \\
\text { Notes }\end{array}$} \\
\hline & & Mission & $\begin{array}{l}\text { Technical } \\
\text { Maturity }\end{array}$ & Environmental & $\begin{array}{c}\text { Engineering / } \\
\text { Design }\end{array}$ & Operation & Regulatory & Stakeholder & Safety & Radiological & & & \\
\hline 15 & $\begin{array}{l}\text { DOE lack of support } \\
\text { of required budget } \\
\text { and schedule may } \\
\text { delay new facility } \\
\text { startup. }\end{array}$ & & & & & & & $\mathrm{x}$ & & & $X$ & & $\begin{array}{l}5 \text { month schedule } \\
\text { impact in the first } \\
\text { year. } \\
6 \text { month schedule } \\
\text { impact in the } \\
\text { second year. } \\
5 \text { month schedule } \\
\text { impact in the } \\
\text { third year. } \\
\text { No change. }\end{array}$ \\
\hline 16 & $\begin{array}{l}\text { SRS infrastructure } \\
\text { may not support the } \\
\text { project needs. }\end{array}$ & & & & 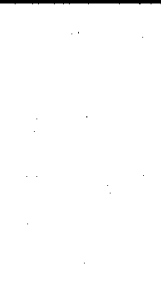 & $\bar{x}$ & & & & & $X$ & & $\begin{array}{l}\$ 22 \text { million cost } \\
\text { increase for } \\
\text { overtime resulting } \\
\text { from staffing } \\
\text { delays. } \\
\text { No change. }\end{array}$ \\
\hline
\end{tabular}




\begin{tabular}{|c|c|c|c|c|c|c|c|c|c|c|c|c|c|}
\hline \multirow{2}{*}{$\begin{array}{l}\text { ID } \\
\text { No }\end{array}$} & \multirow{2}{*}{$\begin{array}{c}\text { Small Tank TPB } \\
\text { Precipitation } \\
\text { Uncertainty } \\
\text { Statement } \\
\end{array}$} & \multicolumn{9}{|c|}{$\begin{array}{c}\text { Areas of } \\
\text { Uncertainty }\end{array}$} & \multirow[b]{2}{*}{1} & \multirow[b]{2}{*}{2} & \multirow[b]{2}{*}{$\begin{array}{c}\text { Explanatory } \\
\text { Notes }\end{array}$} \\
\hline & & Mission & $\begin{array}{l}\text { Technical } \\
\text { Maturity }\end{array}$ & Environmental & $\begin{array}{c}\text { Engineering / } \\
\text { Design }\end{array}$ & Operation & Regulatory & Stakeholder & Safety & Radiological & & & \\
\hline 17 & $\begin{array}{l}\text { Waste removal is } \\
\text { being accelerated. } \\
\text { May conflict with } \\
\text { preferred } \\
\text { alternatives or } \\
\text { preferred alternative } \\
\text { may divert resources } \\
\text { from waste removal } \\
\text { project. }\end{array}$ & $\mathrm{x}$ & & & & & & & & & $X$ & & $\begin{array}{l}\text { No additional cost } \\
\text { or schedule } \\
\text { impact beyond } \\
\text { item } 15 \text {. } \\
\text { No change. }\end{array}$ \\
\hline
\end{tabular}




\begin{tabular}{|c|c|c|c|c|c|c|c|c|c|c|c|c|c|}
\hline \multirow{2}{*}{$\begin{array}{l}\text { ID } \\
\text { No }\end{array}$} & \multirow{2}{*}{$\begin{array}{c}\text { Small Tank TPB } \\
\text { Precipitation } \\
\text { Uncertainty } \\
\text { Statement }\end{array}$} & \multicolumn{9}{|c|}{$\begin{array}{c}\text { Areas of } \\
\text { Uncertainty }\end{array}$} & \multirow[b]{2}{*}{1} & \multirow[b]{2}{*}{2} & \multirow[b]{2}{*}{$\begin{array}{c}\text { Explanatory } \\
\text { Notes }\end{array}$} \\
\hline & & Mission & $\begin{array}{l}\text { Technical } \\
\text { Maturity }\end{array}$ & Environmental & $\begin{array}{c}\text { Engineering / } \\
\text { Design }\end{array}$ & Operation & Regulatory & Stakeholder & $\begin{array}{l}\text { Safety } \\
\text { Sal }\end{array}$ & Radiological & & & \\
\hline 18 & $\begin{array}{l}\text { Pressure on 'old' } \\
\text { infrastructure will } \\
\text { increase, } \\
\text { endangering } \\
\text { schedule due to three } \\
\text { fold increase in flow } \\
\text { requirements from } \\
\text { HTF and FTF. This } \\
\text { would endanger } \\
\text { performance of } \\
\text { infrastructure. }\end{array}$ & & & 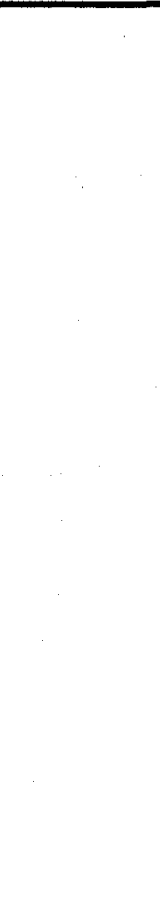 & & $\mathrm{X}$ & & & & & $X$ & & $\begin{array}{l}9 \text { month delay in } \\
\text { completing salt } \\
\text { removal from a } \\
\text { production } \\
\text { schedule delay to } \\
\text { reach salt solution } \\
\text { feed rate } \\
\text { assumption. } \\
\text { Basis: } 50 \% \\
\text { material } \\
\text { movement in the } \\
\text { first year results } \\
\text { in } 6 \text { months and } \\
75 \% \text { material } \\
\text { movement in the } \\
\text { second year } \\
\text { results in } 3 \\
\text { months. } \\
\text { No change. }\end{array}$ \\
\hline 19 & $\begin{array}{l}\text { Improper contract } \\
\text { strategy for design } \\
\text { work may impact the } \\
\text { schedule. }\end{array}$ & & & & $\mathrm{x}$ & & & & & & $X$ & & $\begin{array}{l}6 \text { month delay in } \\
\text { completing } \\
\text { conceptual } \\
\text { design. } \\
\text { No change. }\end{array}$ \\
\hline
\end{tabular}




\begin{tabular}{|c|c|c|c|c|c|c|c|c|c|c|c|c|c|}
\hline \multirow{2}{*}{$\begin{array}{l}\text { ID } \\
\text { No }\end{array}$} & \multirow{2}{*}{$\begin{array}{c}\text { Small Tank TPB } \\
\text { Precipitation } \\
\text { Uncertainty } \\
\text { Statement } \\
\end{array}$} & \multicolumn{9}{|c|}{$\begin{array}{l}\text { Areas of } \\
\text { Uncertainty }\end{array}$} & \multirow[b]{2}{*}{1} & \multirow[b]{2}{*}{2} & \multirow[b]{2}{*}{$\begin{array}{c}\text { Explanatory } \\
\text { Notes }\end{array}$} \\
\hline & & Mission & $\begin{array}{l}\text { Technical } \\
\text { Maturity }\end{array}$ & Environmental & $\begin{array}{c}\begin{array}{c}\text { Engineering/ } \\
\text { Design }\end{array} \\
\end{array}$ & Operation & Regulatory & Stakeholder & Safety & Radiological & & & \\
\hline 20 & $\begin{array}{l}\text { Research and } \\
\text { development work } \\
\text { performed must be } \\
\text { coordinated with the } \\
\text { design effort. }\end{array}$ & & & & $\mathrm{x}$ & & & & & & $X$ & & $\begin{array}{l}6 \text { month delay in } \\
\text { completing } \\
\text { preliminary } \\
\text { design. } \\
\text { No change. } \\
\end{array}$ \\
\hline 21 & $\begin{array}{l}\text { A clearly defined } \\
\text { safety strategy } \\
\text { should be agreed to } \\
\text { by the end of } \\
\text { conceptual design to } \\
\text { preclude schedule } \\
\text { impacts. }\end{array}$ & & & & & & & & $\bar{x}$ & & $\mathrm{X}$ & & $\begin{array}{l}2 \text { month delay in } \\
\text { start of } \\
\text { preliminary } \\
\text { design. } \\
\text { No change. }\end{array}$ \\
\hline 22 & $\begin{array}{l}\text { High source term } \\
\text { with credible release } \\
\text { mechanisms will } \\
\text { concern public. }\end{array}$ & & & & & & & & $\mathrm{x}$ & & $\mathrm{X}$ & & $\begin{array}{l}\text { Within the } \\
\text { existing estimate. } \\
\text { No change. }\end{array}$ \\
\hline 23 & $\begin{array}{l}\text { Inefficiency in the } \\
\text { wash cycle results in } \\
\text { an increase in } \\
\text { NaTPB } \\
\text { consumption. }\end{array}$ & & & & $\mathrm{x}$ & & & & & & $\mathrm{X}$ & & $\begin{array}{l}\$ 25 \text { million cost } \\
\text { increase. } \\
\text { ORNL/TM-1999/234 }\end{array}$ \\
\hline
\end{tabular}




\begin{tabular}{|c|c|c|c|c|c|c|c|c|c|c|c|c|c|}
\hline \multirow{2}{*}{$\begin{array}{l}\text { ID } \\
\text { No }\end{array}$} & \multirow{2}{*}{$\begin{array}{c}\text { Small Tank TPB } \\
\text { Precipitation } \\
\text { Uncertainty } \\
\text { Statement } \\
\end{array}$} & \multicolumn{9}{|c|}{$\begin{array}{c}\text { Areas of } \\
\text { Uncertainty }\end{array}$} & \multirow[b]{2}{*}{1} & \multirow[b]{2}{*}{2} & \multirow[b]{2}{*}{$\begin{array}{c}\text { Explanatory } \\
\text { Notes }\end{array}$} \\
\hline & & Mission & $\begin{array}{l}\text { Technical } \\
\text { Maturity }\end{array}$ & Environmental & $\begin{array}{c}\begin{array}{c}\text { Engineering/ } \\
\text { Designg }\end{array} \\
\end{array}$ & Operation & Regulatory & Stakeholder & Safety & Radiological & & & \\
\hline 24 & $\begin{array}{l}\text { Ability to recycle } \\
\text { wash water reduces } \\
\text { the volume of } \\
\text { saltstone produced. }\end{array}$ & & & & & $\mathrm{X}$ & & & & & $X$ & & $\begin{array}{l}7.8 \text { million } \\
\text { gallons of } \\
\text { saltstone } \\
\text { reduction at } \$ 4 \\
\text { per gallon results } \\
\text { in a cost savings } \\
\text { of } \$ 30 \text { million } \\
\text { and saving the } \\
\text { cost of one vault } \\
\text { of } \$ 9 \text { million. } \\
\text { WSRC-RP-99- } \\
00006\end{array}$ \\
\hline
\end{tabular}



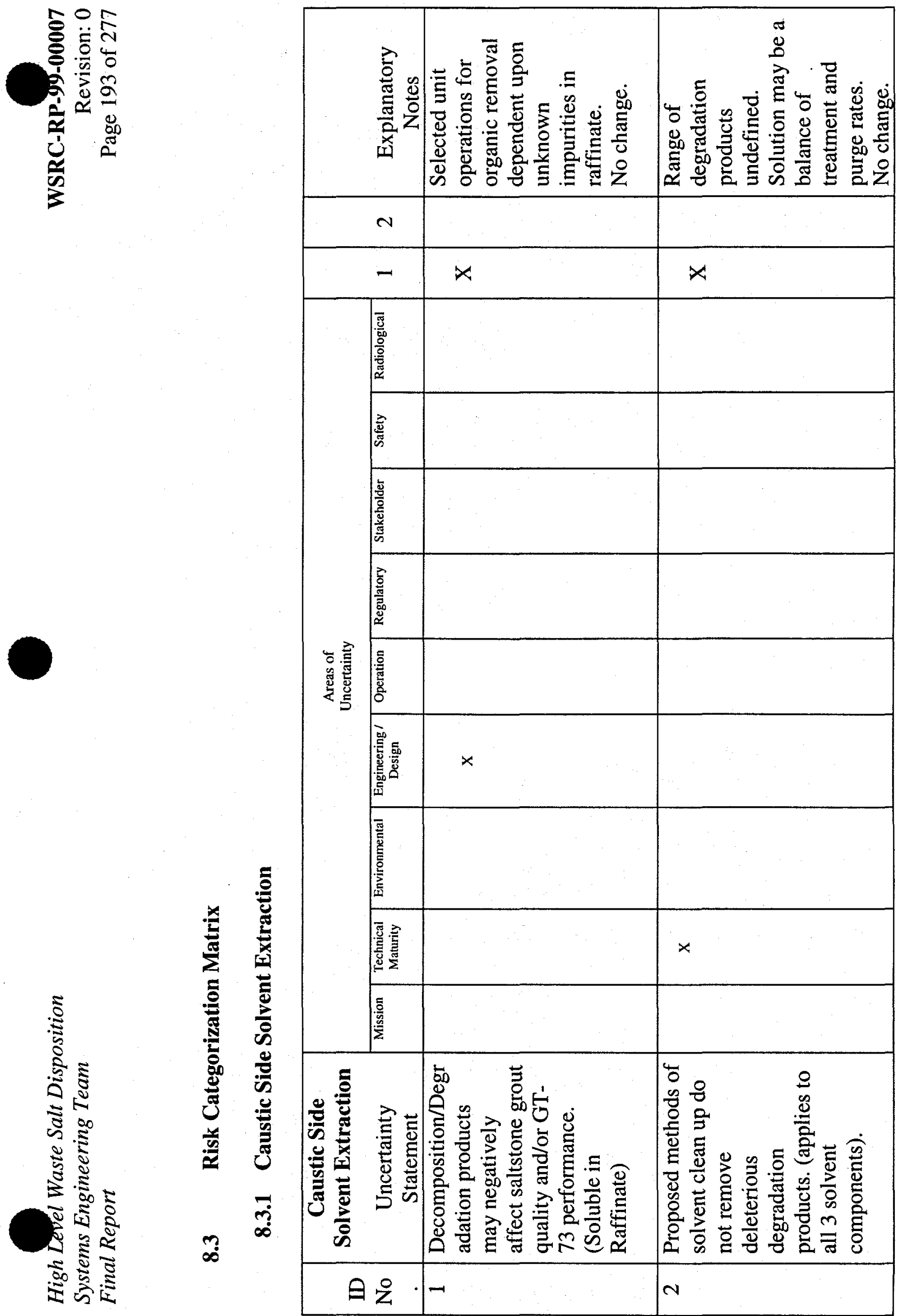
譬

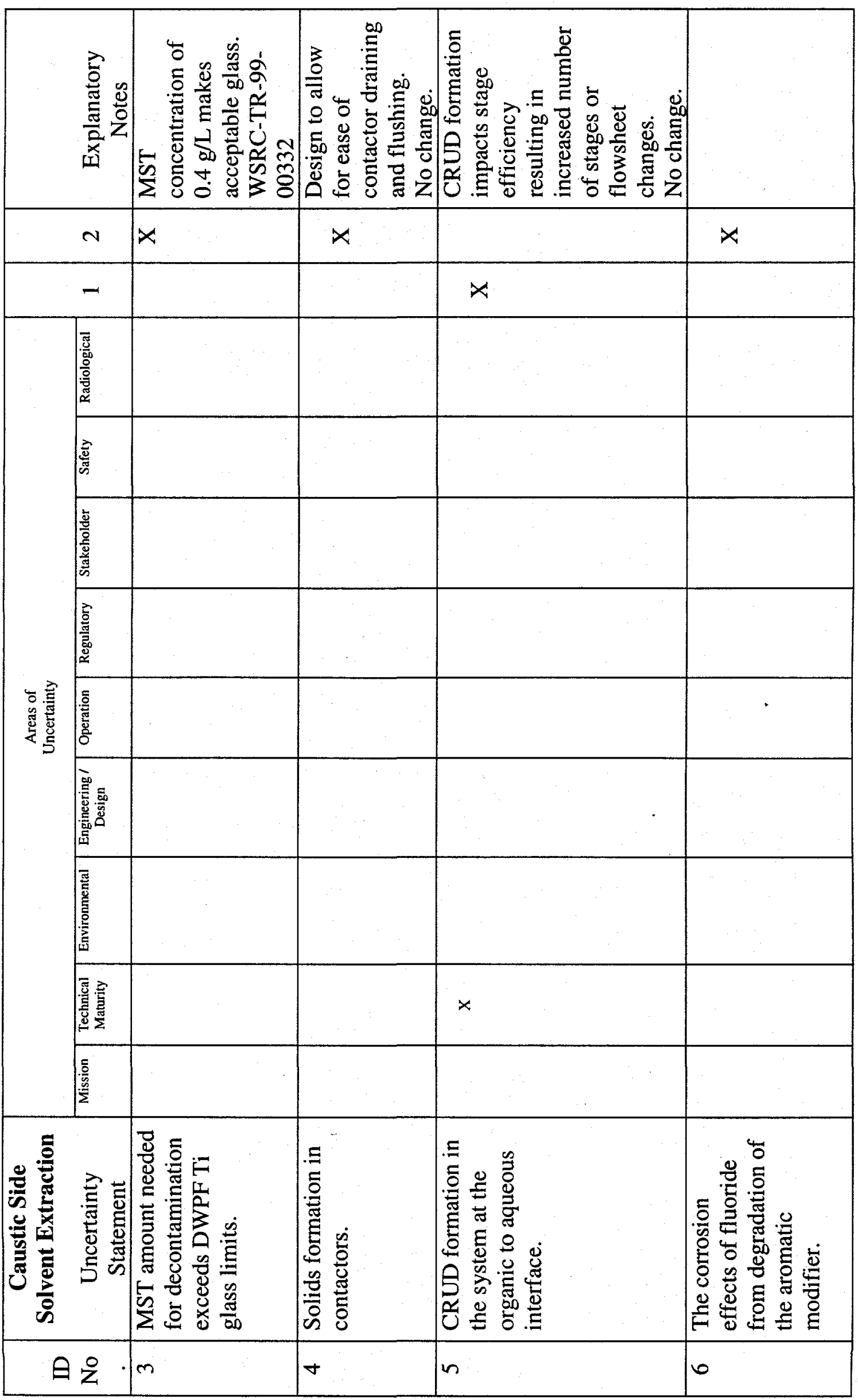




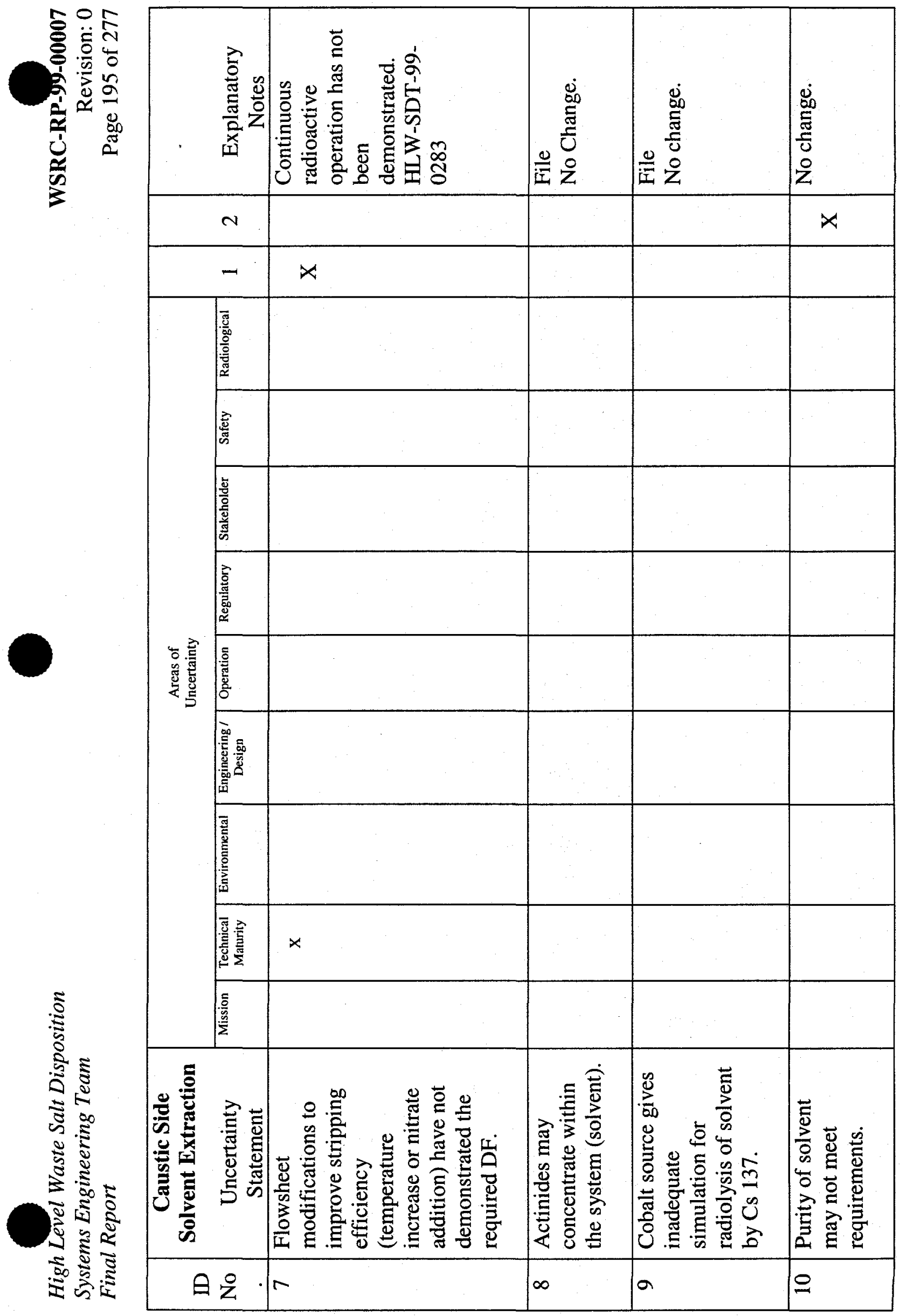


Page 196 of 277

\begin{tabular}{|c|c|c|c|c|c|c|c|c|c|c|c|c|c|}
\hline \multirow{2}{*}{$\begin{array}{l}\text { ID } \\
\text { No }\end{array}$} & \multirow{2}{*}{$\begin{array}{c}\text { Caustic Side } \\
\text { Solvent Extraction } \\
\text { Uncertainty } \\
\text { Statement } \\
\end{array}$} & \multicolumn{9}{|c|}{$\begin{array}{l}\text { Areas of } \\
\text { Uncertainty }\end{array}$} & \multirow[b]{2}{*}{1} & \multirow[b]{2}{*}{2} & \multirow[b]{2}{*}{$\begin{array}{c}\text { Explanatory } \\
\text { Notes }\end{array}$} \\
\hline & & Mission & $\begin{array}{l}\text { Technical } \\
\text { Maturity }\end{array}$ & Environmental & $\begin{array}{l}\text { Engineering/ } \\
\text { Design }\end{array}$ & Operation & Regulatory & Stakeholder & Safety & Radiological & & & \\
\hline 11 & $\begin{array}{l}\text { Modifier in solvent } \\
\text { will not be } \\
\text { commercially } \\
\text { available. }\end{array}$ & & & & & & & & & & & & $\begin{array}{l}\text { File } \\
\text { No change. }\end{array}$ \\
\hline 12 & $\begin{array}{l}\text { Existing transfer } \\
\text { lines and tank size } \\
\text { and drain back not } \\
\text { adequate, and leads } \\
\text { to inefficiencies. }\end{array}$ & & & & & & & & & & & $X$ & No change. \\
\hline 13 & $\begin{array}{l}\text { Flexibility of output } \\
\text { stream in coupling to } \\
\text { DWPF. }\end{array}$ & & & & & & & & & & & & $\begin{array}{l}\text { File } \\
\text { Purer feed stream } \\
\text { does not effect } \\
\text { Tank Farm feed } \\
\text { preparation rate. } \\
\text { No change. } \\
\end{array}$ \\
\hline 14 & $\begin{array}{l}\text { Centrifuge contactor } \\
\text { reaches steady state } \\
\text { very quickly } \\
\text { (minutes-hours). } \\
\text { Easy to shut down } \\
\text { on weekends and } \\
\text { restart. }\end{array}$ & & & & & & & & & & & & $\begin{array}{l}\text { File } \\
\text { No change. }\end{array}$ \\
\hline
\end{tabular}




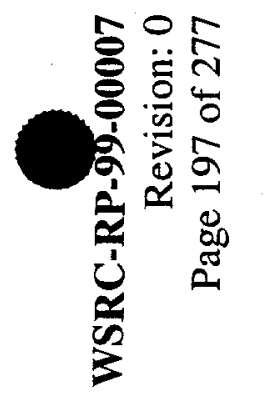

\begin{tabular}{|c|c|c|c|c|c|}
\hline & 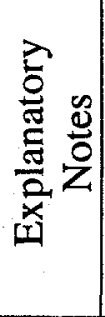 & 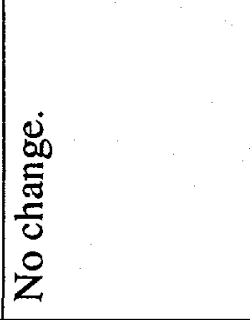 & 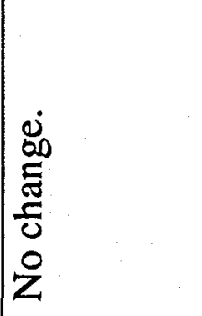 & 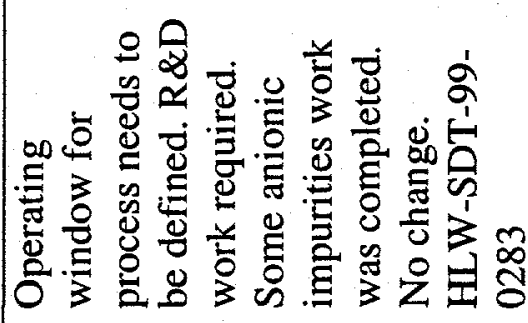 & 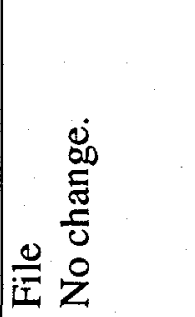 \\
\hline & $N$ & 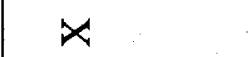 & $x$ & & \\
\hline & - & & & $\not$ & \\
\hline \multirow{9}{*}{ 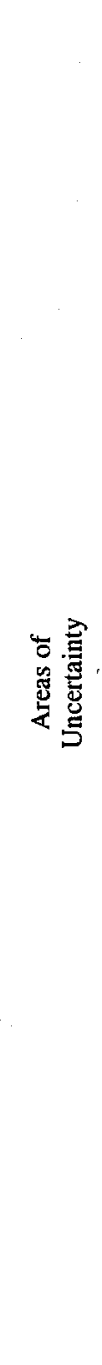 } & 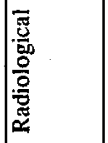 & & & & \\
\hline & $\frac{2}{\frac{d}{2}}$ & & & & \\
\hline & 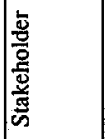 & & & . & \\
\hline & 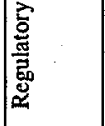 & & & & \\
\hline & 点 & & & $x$ & \\
\hline & 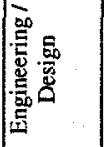 & & & & \\
\hline & 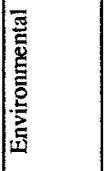 & & & & \\
\hline & 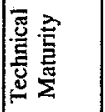 & & & & \\
\hline & $\mid \begin{array}{l}\frac{5}{6} \\
\frac{5}{2}\end{array}$ & & & & \\
\hline 吾 & 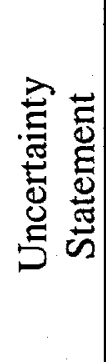 & 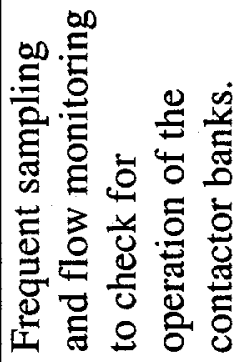 & 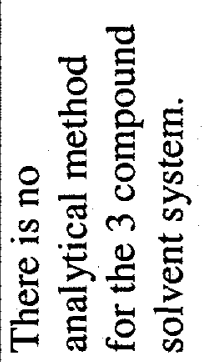 & 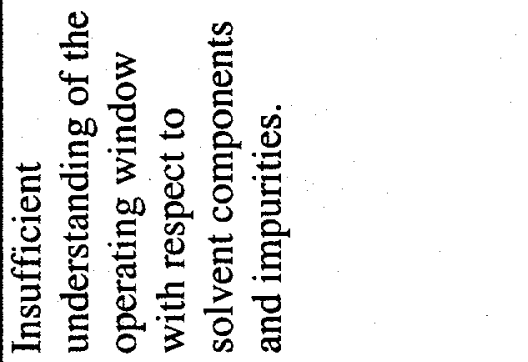 & 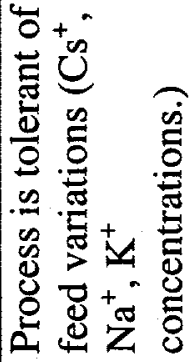 \\
\hline & $\stackrel{\circ}{Z}$ & $n$ & 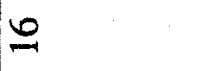 & $\beth$ & $\infty$ \\
\hline
\end{tabular}




\begin{tabular}{|c|c|c|c|c|c|c|c|c|c|c|c|c|c|}
\hline \multirow{2}{*}{$\begin{array}{l}\text { ID } \\
\text { No }\end{array}$} & \multirow{2}{*}{$\begin{array}{c}\text { Caustic Side } \\
\text { Solvent Extraction } \\
\text { Uncertainty } \\
\text { Statement }\end{array}$} & \multicolumn{9}{|c|}{$\begin{array}{l}\text { Areas of } \\
\text { Uncertainty }\end{array}$} & \multirow[b]{2}{*}{1} & \multirow[b]{2}{*}{2} & \multirow[b]{2}{*}{$\begin{array}{c}\text { Explanatory } \\
\text { Notes }\end{array}$} \\
\hline & & Mission & $\begin{array}{l}\text { Technical } \\
\text { Maturity }\end{array}$ & Environmental & $\begin{array}{c}\text { Engineering / } \\
\text { Design }\end{array}$ & Operation & $\begin{array}{l}\text { Regulatory } \\
\end{array}$ & \begin{tabular}{|l|} 
Stakeholder \\
\end{tabular} & Safety & $\begin{array}{l}\text { Radiological } \\
\end{array}$ & & & \\
\hline 19 & $\begin{array}{l}\text { Favorable impacts } \\
\text { on DWPF AB. }\end{array}$ & & & & & & & & & & & & $\begin{array}{l}\text { File } \\
\text { No change. }\end{array}$ \\
\hline 20 & $\begin{array}{l}\text { High reliability of } \\
\text { canyon centrifugal } \\
\text { six-pack. Only } 1 \\
\text { change in }>30 \text { years. }\end{array}$ & & & & & & & & & & & & $\begin{array}{l}\text { File } \\
\text { No change. }\end{array}$ \\
\hline 21 & $\begin{array}{l}\text { Recovery from } \\
\text { process upsets } \\
\text { (Phase inversions, } \\
\text { failures,...) }\end{array}$ & & & & & & & & & & & $\mathrm{X}$ & No change. \\
\hline 22 & $\begin{array}{l}\text { Solids on filters will } \\
\text { not dissolve or } \\
\text { would be difficult to } \\
\text { dissolve in oxalic } \\
\text { acid. }\end{array}$ & & & & & & & & & & & & $\begin{array}{l}\text { File } \\
\text { No change. }\end{array}$ \\
\hline
\end{tabular}




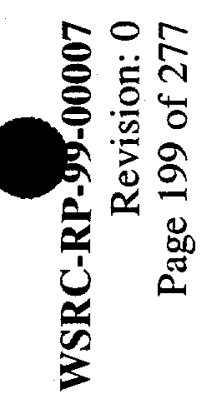

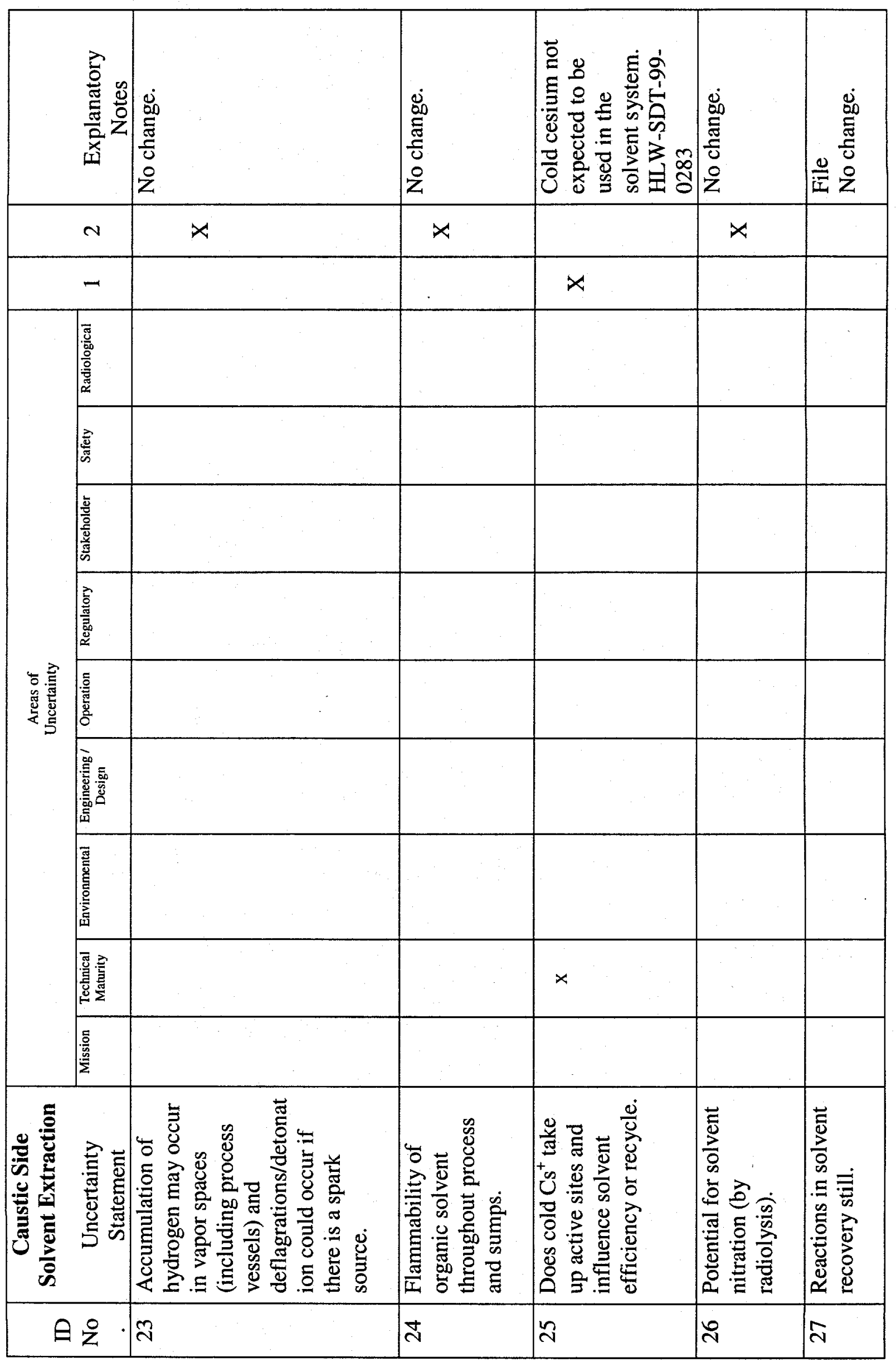




\begin{tabular}{|c|c|c|c|c|c|c|}
\hline \multirow[t]{11}{*}{ 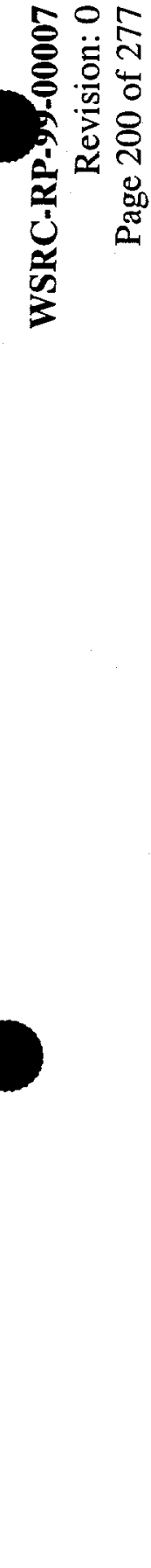 } & & 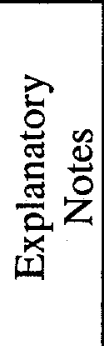 & 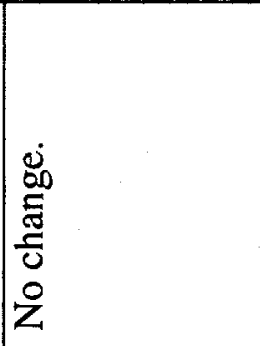 & 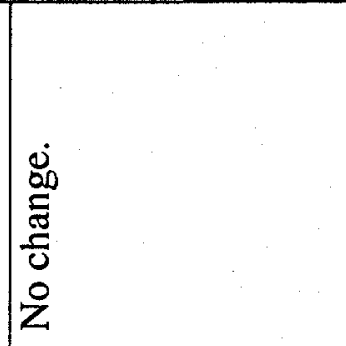 & 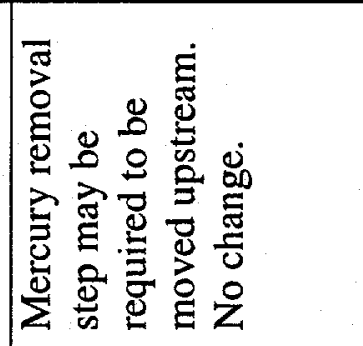 & 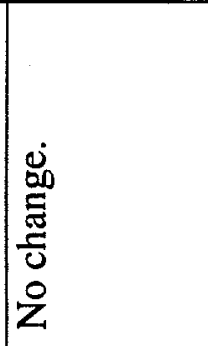 \\
\hline & & $N$ & $x$ & $x$ & & $x$ \\
\hline & & - & & & $\rtimes$ & \\
\hline & \multirow{9}{*}{ 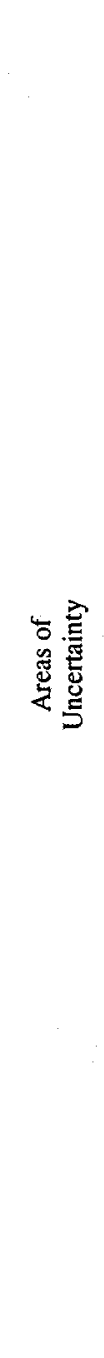 } & 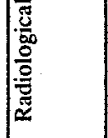 & & & & \\
\hline & & 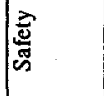 & & & & \\
\hline & & 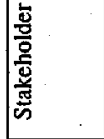 & & & & \\
\hline & & 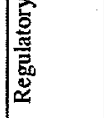 & & & & \\
\hline & & 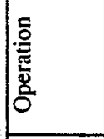 & & & & \\
\hline & & 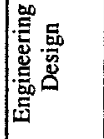 & & & & \\
\hline & & 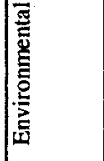 & & & & \\
\hline & & 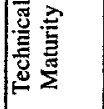 & & & $x$ & \\
\hline \multirow{3}{*}{ 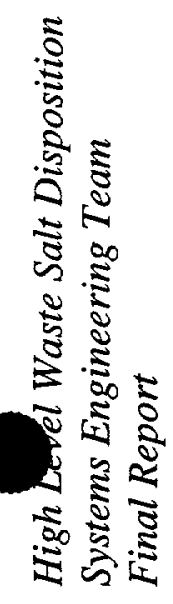 } & & 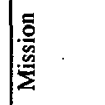 & & & & \\
\hline & 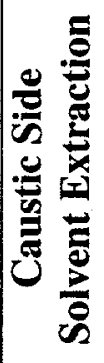 & 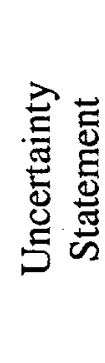 & 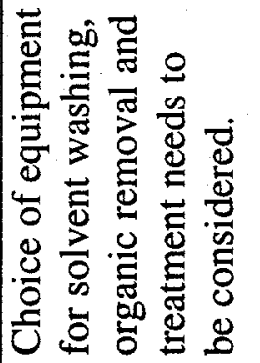 & 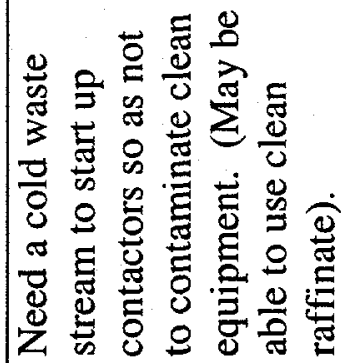 & 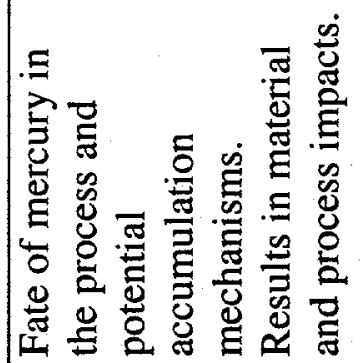 & 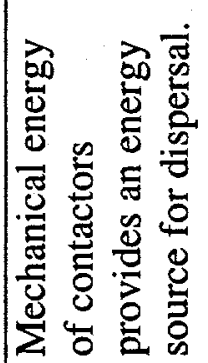 \\
\hline & & $\stackrel{0}{z}$ & $\stackrel{\infty}{\sim}$ & নি & 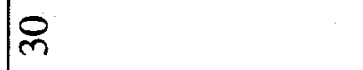 & $\bar{m}$ \\
\hline
\end{tabular}




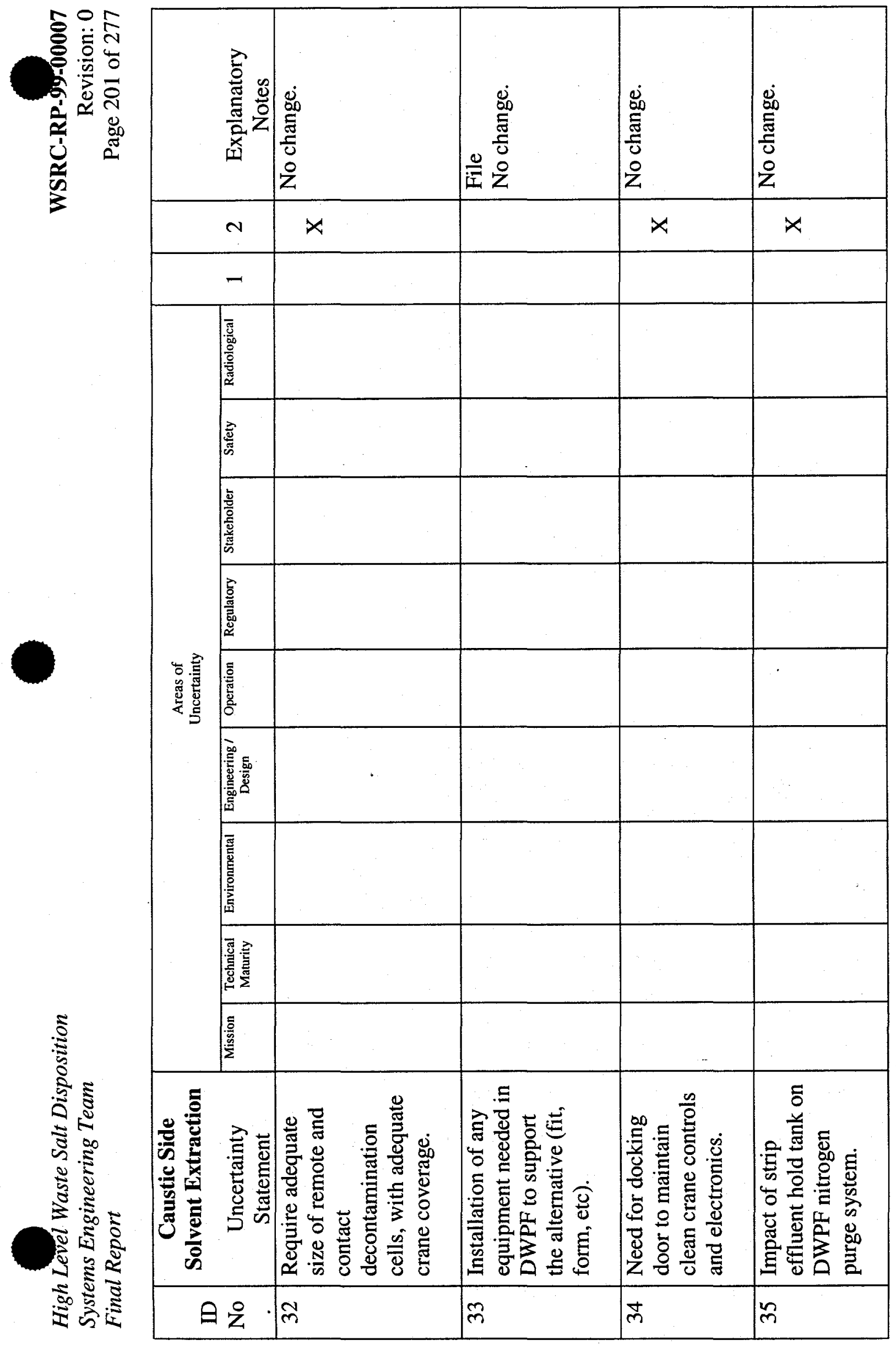


Page 202 of 277

\begin{tabular}{|c|c|c|c|c|c|c|c|c|c|c|c|c|c|}
\hline \multirow{2}{*}{$\begin{array}{l}\text { ID } \\
\text { No }\end{array}$} & \multirow{2}{*}{$\begin{array}{c}\text { Caustic Side } \\
\text { Solvent Extraction } \\
\text { Uncertainty } \\
\text { Statement } \\
\end{array}$} & \multicolumn{9}{|c|}{$\begin{array}{c}\text { Areas of } \\
\text { Uncertainty }\end{array}$} & \multirow[b]{2}{*}{1} & \multirow[b]{2}{*}{2} & \multirow[b]{2}{*}{$\begin{array}{l}\text { Explanatory } \\
\text { Notes }\end{array}$} \\
\hline & & Mission & $\begin{array}{l}\text { Technical } \\
\text { Maturity }\end{array}$ & Environmental & $\begin{array}{l}\text { Engineering / } \\
\text { Design }\end{array}$ & Operation & Regulatory & Stakeholder & Safety & Radiological & & & \\
\hline 36 & $\begin{array}{l}\text { Strategy to minimize } \\
\text { contactor shut } \\
\text { downs and } \\
\text { interruptions ie. } \\
\text { routine utility upsets } \\
\text { do not result in } \\
\text { process upsets }\end{array}$ & & & & & & & & & & & $\mathrm{X}$ & No change. \\
\hline 37 & $\begin{array}{l}\text { Adequate process } \\
\text { instrumentation to } \\
\text { detect process upsets } \\
\text { and perform routine } \\
\text { monitoring. }\end{array}$ & & & & & & & & & & & $X$ & No change. \\
\hline 38 & $\begin{array}{l}\text { Need for additional } \\
\text { equipment/design } \\
\text { for the testing phase } \\
\text { (ie. - start-up) }\end{array}$ & & & & & & & & & & & $\mathrm{X}$ & No change. \\
\hline 39 & $\begin{array}{l}\text { Strategy for disposal } \\
\text { of special cold } \\
\text { chemicals from cold } \\
\text { chemical start-up } \\
\text { tests. }\end{array}$ & & & & & & & & & & & & $\begin{array}{l}\text { File } \\
\text { No change. }\end{array}$ \\
\hline 40 & $\begin{array}{l}\text { Inability to clean } \\
\text { the solvent }\end{array}$ & & & & & & & & & & & & $\begin{array}{l}\text { File } \\
\text { No change. }\end{array}$ \\
\hline
\end{tabular}


Page 203 of 277

\begin{tabular}{|c|c|c|c|c|c|c|c|c|c|c|c|c|c|}
\hline \multirow{2}{*}{$\begin{array}{l}\text { ID } \\
\text { No }\end{array}$} & \multirow{2}{*}{$\begin{array}{c}\text { Caustic Side } \\
\text { Solvent Extraction } \\
\text { Uncertainty } \\
\text { Statement } \\
\end{array}$} & \multicolumn{9}{|c|}{$\begin{array}{l}\text { Areas of } \\
\text { Uncertainty }\end{array}$} & \multirow[b]{2}{*}{1} & \multirow[b]{2}{*}{2} & \multirow[b]{2}{*}{$\begin{array}{c}\begin{array}{c}\text { Explanatory } \\
\text { Notes }\end{array} \\
\end{array}$} \\
\hline & & Mission & $\begin{array}{l}\text { Technical } \\
\text { Maturity }\end{array}$ & Environmental & $\begin{array}{c}\text { Engineering/ } \\
\text { Design }\end{array}$ & Operation & Regulatory & Stakeholder & Safety & Radiological & & & \\
\hline 41 & $\begin{array}{l}\text { Inadvertent transfer } \\
\text { of organics to } \\
\text { Saltstone feed } \\
\text { storage } \operatorname{tank}(\mathrm{s})\end{array}$ & & & & & & & & & & & & $\begin{array}{l}\text { File } \\
\text { No change. }\end{array}$ \\
\hline 42 & $\begin{array}{l}\text { Addition of organic } \\
\text { removal for raffinate } \\
\text { may be required }\end{array}$ & & & & & & & & & & & $X$ & No change. \\
\hline 43 & $\begin{array}{l}\text { Solvent dissolving } \\
\text { undesired } \\
\text { compounds from } \\
\text { aqueous stream } \\
\text { impacts quality of } \\
\text { feed stream to } \\
\text { DWPF. }\end{array}$ & & & & $\mathrm{x}$ & & & & & & $\mathrm{X}$ & & $\begin{array}{l}\text { Selected unit } \\
\text { operations } \\
\text { dependent upon } \\
\text { strip effluent. } \\
\text { No change. }\end{array}$ \\
\hline 44 & $\begin{array}{l}\text { Combination of } \mathrm{Al} \text {, } \\
\text { Silica, and Fe affect } \\
\text { the ability to run this } \\
\text { process. }\end{array}$ & & $\mathrm{x}$ & & & & & & & & $X$ & & $\begin{array}{l}\text { These species } \\
\text { should be } \\
\text { included in future } \\
\text { laboratory tests. } \\
\text { No change. }\end{array}$ \\
\hline
\end{tabular}


Page 204 of 277

\begin{tabular}{|c|c|c|c|c|c|c|c|c|c|c|c|c|c|}
\hline \multirow{2}{*}{$\begin{array}{l}\text { ID } \\
\text { No }\end{array}$} & \multirow{2}{*}{$\begin{array}{c}\text { Caustic Side } \\
\text { Solvent Extraction } \\
\text { Uncertainty } \\
\text { Statement }\end{array}$} & \multicolumn{9}{|c|}{$\begin{array}{c}\text { Areas of } \\
\text { Uncertainty }\end{array}$} & \multirow[b]{2}{*}{1} & \multirow[b]{2}{*}{2} & \multirow[b]{2}{*}{$\begin{array}{l}\text { Explanatory } \\
\text { Notes }\end{array}$} \\
\hline & & Mission & $\begin{array}{l}\text { Technical } \\
\text { Maturity }\end{array}$ & Environmental & $\begin{array}{c}\begin{array}{c}\text { Engineering } / \\
\text { Design }\end{array} \\
\text { (n) }\end{array}$ & Operation & Regulatory & Stakeholder & Safety & Radiological & & & \\
\hline 45 & $\begin{array}{l}\text { Difficulty in } \\
\text { separating the } \\
\text { organic stream from } \\
\text { the aqueous stream. }\end{array}$ & & & & $x$ & & & & & & $X$ & & $\begin{array}{l}\text { Assumed } \\
\text { contactor } \\
\text { efficiency of } 95 \% \\
\text { requires } \\
\text { confirmation. } \\
\text { Probably } \\
\text { important cause } \\
\text { of solvent loss. } \\
\text { No change. } \\
\end{array}$ \\
\hline 46 & $\begin{array}{l}\text { Excessive solvent } \\
\text { degradation due to } \\
\text { radiolysis }\end{array}$ & & & & & & & & & & & & $\begin{array}{l}\text { File } \\
\text { No change. }\end{array}$ \\
\hline 47 & $\begin{array}{l}\text { Complex unknown } \\
\text { process } \\
\text { measurement } \\
\text { techniques will be } \\
\text { required. }\end{array}$ & & 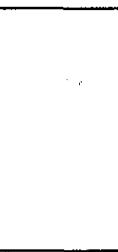 & & & & & & & & & & $\begin{array}{l}\text { File } \\
\text { No change. }\end{array}$ \\
\hline 48 & $\begin{array}{l}\text { Difficulty in } \\
\text { filtration of sludge } \\
\text { and/or MST will } \\
\text { produce low filtrate } \\
\text { flow rates and } \\
\text { require frequent } \\
\text { cleaning }\end{array}$ & & $\mathrm{x}$ & & & & & & & & $\mathrm{X}$ & & $\begin{array}{l}\text { R\&D work has } \\
\text { confirmed this } \\
\text { risk. } \\
\text { WSRC-TR-99- } \\
00346\end{array}$ \\
\hline
\end{tabular}


Page 205 of 277

\begin{tabular}{|c|c|c|c|c|c|c|c|c|c|c|c|c|c|}
\hline \multirow{2}{*}{$\begin{array}{l}\text { ID } \\
\text { No }\end{array}$} & \multirow{2}{*}{$\begin{array}{c}\text { Caustic Side } \\
\text { Solvent Extraction } \\
\text { Uncertainty } \\
\text { Statement }\end{array}$} & \multicolumn{9}{|c|}{$\begin{array}{l}\text { Areas of } \\
\text { Uncertainty }\end{array}$} & \multirow[b]{2}{*}{1} & \multirow[b]{2}{*}{2} & \multirow[b]{2}{*}{$\begin{array}{c}\text { Explanatory } \\
\text { Notes }\end{array}$} \\
\hline & & Mission & $\begin{array}{l}\text { Technical } \\
\text { Maturity }\end{array}$ & Environmental & $\begin{array}{c}\text { Engineering I } \\
\text { Design }\end{array}$ & Operation & Regulatory & Stakeholder & Safety & Radiological & & & \\
\hline 49 & $\begin{array}{l}\text { Difficulty in } \\
\text { resuspending MST } \\
\text { after long quiescent } \\
\text { period }\end{array}$ & & & & $\mathrm{X}$ & & & & & & $\mathrm{X}$ & & $\begin{array}{l}\text { After } 60 \text { days of } \\
\text { settling, } \\
\text { resuspension was } \\
\text { not achieved with } \\
\text { proposed } \\
\text { flowsheet design. } \\
\text { ORNL-TM- } \\
\text { 1999/166 }\end{array}$ \\
\hline 50 & $\begin{array}{l}\text { The technical } \\
\text { immaturity of the } \\
\text { "Solvent System" } \\
\text { will result in failure } \\
\text { of the process. }\end{array}$ & & & & & & & & & & & & $\begin{array}{l}\text { File } \\
\text { No change. }\end{array}$ \\
\hline 51 & $\begin{array}{l}\text { HLW cannot support } \\
\text { tank blending } \\
\text { strategies for Cs (or } \\
\text { other species) to } \\
\text { support process } \\
\text { requirements. }\end{array}$ & & & & & & & & & & & $X$ & No change. \\
\hline 52 & $\begin{array}{l}\text { GT73 resin will be } \\
\text { selective to Co60 or } \\
\text { other trace } \\
\text { radioisotopes. }\end{array}$ & & & & & & & & & & & & File \\
\hline 53 & $\begin{array}{l}\text { Contacting of GT-73 } \\
\text { with organics }\end{array}$ & & & & & & . & & & & & & $\begin{array}{l}\text { File } \\
\text { No change. }\end{array}$ \\
\hline
\end{tabular}




\begin{tabular}{|c|c|c|c|c|c|c|c|c|c|c|c|c|c|}
\hline \multirow{2}{*}{$\begin{array}{l}\text { ID } \\
\text { No }\end{array}$} & \multirow{2}{*}{$\begin{array}{c}\text { Caustic Side } \\
\text { Solvent Extraction } \\
\text { Uncertainty } \\
\text { Statement }\end{array}$} & \multicolumn{9}{|c|}{$\begin{array}{c}\text { Areas of } \\
\text { Uncertainty }\end{array}$} & \multirow[b]{2}{*}{1} & \multirow[b]{2}{*}{2} & \multirow[b]{2}{*}{$\begin{array}{c}\text { Explanatory } \\
\text { Notes }\end{array}$} \\
\hline & & Mission & $\begin{array}{l}\text { Technical } \\
\text { Maturity }\end{array}$ & Environmental & $\begin{array}{c}\text { Engineering / } \\
\text { Design }\end{array}$ & Operation & Regulatory & Stakeholder & Safety & Radiological & & & \\
\hline 54 & $\begin{array}{l}\text { Solid waste has no } \\
\text { disposal routes for } \\
\text { spent solvent }\end{array}$ & & & & & & & & & & & $\mathrm{X}$ & No change. \\
\hline 55 & $\begin{array}{l}\text { Potential nitration of } \\
\text { organics in the strip } \\
\text { stream }\end{array}$ & & & & & & & & & & & & $\begin{array}{l}\text { File } \\
\text { No change. }\end{array}$ \\
\hline 56 & $\begin{array}{l}\text { Fire in the solvent } \\
\text { extraction process }\end{array}$ & & & & & & & & & & & & $\begin{array}{l}\text { File } \\
\text { No change. }\end{array}$ \\
\hline 57 & $\begin{array}{l}\text { Evaporator } \\
\text { deflagration can } \\
\text { occur }\end{array}$ & & & & & & & & & & & & $\begin{array}{l}\text { File } \\
\text { No change. }\end{array}$ \\
\hline 58 & $\begin{array}{l}\text { Personnel exposure } \\
\text { to the toxic } \\
\text { solvent/diluent could } \\
\text { occur }\end{array}$ & & & & & & & & & & & $\mathrm{X}$ & No change. \\
\hline 59 & $\begin{array}{l}\text { By-products would } \\
\text { be carried to } \\
\text { Saltstone in excess } \\
\text { of permit limits }\end{array}$ & t & & . & & & & & & & & & $\begin{array}{l}\text { File } \\
\text { No change. }\end{array}$ \\
\hline
\end{tabular}




\begin{tabular}{|c|c|c|c|c|c|c|c|c|c|c|c|c|c|}
\hline \multirow{2}{*}{$\begin{array}{l}\text { ID } \\
\text { No }\end{array}$} & \multirow{2}{*}{$\begin{array}{c}\text { Caustic Side } \\
\text { Solvent Extraction } \\
\text { Uncertainty } \\
\text { Statement } \\
\end{array}$} & \multicolumn{9}{|c|}{$\begin{array}{l}\text { Areas of } \\
\text { Uncertainty }\end{array}$} & \multirow[b]{2}{*}{1} & \multirow[b]{2}{*}{2} & \multirow[b]{2}{*}{$\begin{array}{c}\text { Explanatory } \\
\text { Notes }\end{array}$} \\
\hline & & Mission & $\begin{array}{l}\text { Technical } \\
\text { Maturity }\end{array}$ & Environmental & $\begin{array}{c}\text { Engineering/ } \\
\text { Design }\end{array}$ & Operation & Regulatory & Stakeholder & Safety & $\begin{array}{l}\text { Radiological } \\
\end{array}$ & & & \\
\hline 60 & $\begin{array}{l}\text { Process chemistry } \\
\text { understanding and } \\
\text { application are still } \\
\text { under development, } \\
\text { resulting in 96-1 } \\
\text { lessons learned not } \\
\text { yet implemented }\end{array}$ & & $\mathrm{x}$ & & & & & & & & $X$ & & $\begin{array}{l}\text { Additional R\&D } \\
\text { work needed to } \\
\text { close out DNFSB } \\
96-1 \text { concerns. } \\
\text { Some anionic } \\
\text { impurities work } \\
\text { was completed. } \\
\text { No change. } \\
\text { HLW-SDT-99- } \\
0283\end{array}$ \\
\hline 61 & $\begin{array}{l}\text { Lack of process data } \\
\text { will lead to a } \\
\text { complex design }\end{array}$ & & & & & 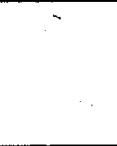 & & & & & & & $\begin{array}{l}\text { File } \\
\text { No change. }\end{array}$ \\
\hline 62 & $\begin{array}{l}\text { Mercury removal } \\
\text { resin (GT-73) will } \\
\text { not work in high } \\
\text { caustic environment } \\
\text { ( }>2 \text { Molar). }\end{array}$ & & & & & & & & & & & & File \\
\hline 63 & $\begin{array}{l}\text { Will not be able to } \\
\text { procure sufficient } \\
\text { extractant quantities }\end{array}$ & & & & & & & & & & & & $\begin{array}{l}\text { File } \\
\text { No change. }\end{array}$ \\
\hline 64 & $\begin{array}{l}\text { Production size } \\
\text { contactors not } \\
\text { commercially } \\
\text { available }\end{array}$ & & & & & & & & & & & & $\begin{array}{l}\text { File } \\
\text { No change. }\end{array}$ \\
\hline
\end{tabular}




\begin{tabular}{|c|c|c|c|c|c|c|c|c|c|c|c|c|c|}
\hline \multirow{2}{*}{$\begin{array}{l}\text { ID } \\
\text { No }\end{array}$} & \multirow{2}{*}{$\begin{array}{c}\text { Caustic Side } \\
\text { Solvent Extraction } \\
\text { Uncertainty } \\
\text { Statement }\end{array}$} & \multicolumn{9}{|c|}{$\begin{array}{l}\text { Areas of } \\
\text { Uncertainty }\end{array}$} & \multirow[b]{2}{*}{1} & \multirow[b]{2}{*}{2} & \multirow[b]{2}{*}{$\begin{array}{l}\text { Explanatory } \\
\text { Notes }\end{array}$} \\
\hline & & $\begin{array}{l}\text { Mission } \\
\end{array}$ & $\begin{array}{l}\text { Tecthical } \\
\text { Maturity }\end{array}$ & Environmental & $\begin{array}{c}\text { Engineering/ } \\
\text { Design }\end{array}$ & Operation & $\begin{array}{l}\text { Regulatory } \\
\text {. }\end{array}$ & Stakeholder & $\begin{array}{l}\text { Safely } \\
\end{array}$ & Radiological & & & \\
\hline 65 & $\begin{array}{l}\text { If facility location is } \\
\text { F Canyon, an } \\
\text { additional transfer } \\
\text { line may be required }\end{array}$ & & & & & & & & & & & & $\begin{array}{l}\text { File } \\
\text { No change. }\end{array}$ \\
\hline 66 & $\begin{array}{l}\text { Based on selected } \\
\text { location operating } \\
\text { facilities will be } \\
\text { impacted. }\end{array}$ & & & & & & & & & & & & $\begin{array}{l}\text { File } \\
\text { No change. }\end{array}$ \\
\hline 67 & $\begin{array}{l}\text { Looks like } \\
\text { reprocessing and } \\
\text { existing facility life } \\
\text { extension. }\end{array}$ & & & & & & & & & & & & $\begin{array}{l}\text { File } \\
\text { No change. }\end{array}$ \\
\hline 68 & $\begin{array}{l}\text { TRU } \\
\text { decontamination } \\
\text { with MST is not } \\
\text { adequate. }\end{array}$ & & $\mathbf{x}$ & & & & & & & & $\mathrm{X}$ & & $\begin{array}{l}\text { Kinetics for } \\
\text { bounding } \\
\text { plutonium is too } \\
\text { slow. } \\
\text { No change. } \\
\text { WSRC-TR-99- } \\
00219 \\
\text { WSRC-TR-99- } \\
00286\end{array}$ \\
\hline
\end{tabular}




\begin{tabular}{|c|c|c|c|c|c|c|c|c|c|c|c|c|c|}
\hline \multirow{2}{*}{$\begin{array}{l}\text { ID } \\
\text { No }\end{array}$} & \multirow{2}{*}{$\begin{array}{c}\text { Caustic Side } \\
\text { Solvent Extraction } \\
\text { Uncertainty } \\
\text { Statement } \\
\end{array}$} & \multicolumn{9}{|c|}{$\begin{array}{l}\text { Areas of } \\
\text { Uncertainty }\end{array}$} & \multirow[b]{2}{*}{1} & \multirow[b]{2}{*}{2} & \multirow[b]{2}{*}{$\begin{array}{c}\text { Explanatory } \\
\text { Notes }\end{array}$} \\
\hline & & $\begin{array}{l}\text { Mission } \\
\end{array}$ & $\begin{array}{l}\text { Technical } \\
\text { Maturity }\end{array}$ & Environmental & $\begin{array}{c}\text { Engineering / } \\
\text { Design }\end{array}$ & Operation & Regulatory & Stakeholder & Safety & Radiological & & & \\
\hline 69 & $\begin{array}{l}\text { TRU } \\
\text { decontamination } \\
\text { may require } \\
\text { excessive MST. }\end{array}$ & & $X$ & & & & & & & & $X$ & & $\begin{array}{l}\text { Equilibrium } \\
\text { capacity shown to } \\
\text { be sufficient by } \\
\text { R\&D. Neptunium } \\
\text { for Tanks } 33 \text { and } \\
34 \text { will require } \\
\text { blending. } \\
\text { No change. } \\
\text { WSRC-TR-99- } \\
\text { 00219 } \\
\text { WSRC-TR-99- } \\
\text { 00286 }\end{array}$ \\
\hline 70 & $\begin{array}{l}\text { The neptunium } \\
\text { content in certain } \\
\text { HLW Tanks may } \\
\text { impact the PA and } \\
\text { WAC. }\end{array}$ & & & & & & & & & & & & $\begin{array}{l}\text { File } \\
\text { No change. }\end{array}$ \\
\hline 71. & $\begin{array}{l}\text { Monitoring GT-73 } \\
\text { performance and } \\
\text { breakthrough. }\end{array}$ & & & & & & & & & & & & File \\
\hline
\end{tabular}




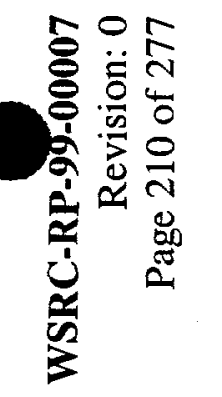

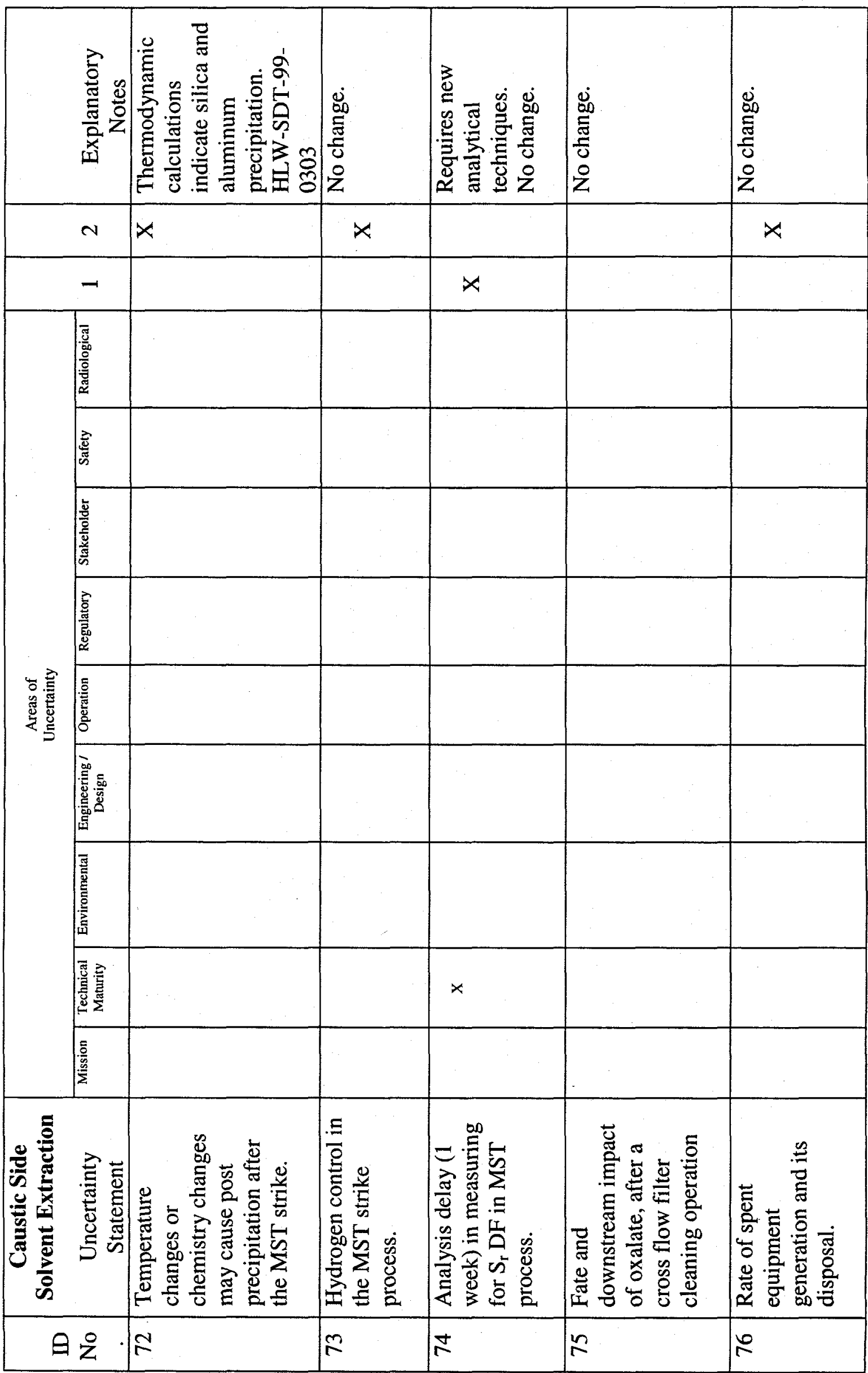




\begin{tabular}{|c|c|c|c|c|c|c|c|c|c|c|c|c|c|}
\hline \multirow{2}{*}{$\begin{array}{l}\text { ID } \\
\text { No }\end{array}$} & \multirow{2}{*}{$\begin{array}{c}\text { Caustic Side } \\
\text { Solvent Extraction } \\
\text { Uncertainty } \\
\text { Statement } \\
\end{array}$} & \multicolumn{9}{|c|}{$\begin{array}{l}\text { Areas of } \\
\text { Uncertainty }\end{array}$} & \multirow[b]{2}{*}{1} & \multirow[b]{2}{*}{2} & \multirow[b]{2}{*}{$\begin{array}{c}\text { Explanatory } \\
\text { Notes }\end{array}$} \\
\hline & & Mission & $\begin{array}{l}\text { Technical } \\
\text { Maturity }\end{array}$ & Environmental & $\begin{array}{c}\text { Engineering } \\
\text { Design }\end{array}$ & Operation & Regulatory & Stakeholder & Safety & Radiological & & & \\
\hline 77 & $\begin{array}{l}\text { Minimize spark } \\
\text { sources in } \mathrm{H}_{2} \text { rich } \\
\text { areas. }\end{array}$ & & & & & & & & & & & $\mathrm{X}$ & No change. \\
\hline 78 & $\begin{array}{l}\text { What is the fate of } \\
\text { Am in the process? }\end{array}$ & & $\mathrm{x}$ & & & & & & & & $\mathrm{X}$ & & $\begin{array}{l}\text { Does MST strike } \\
\text { affect Am } \\
\text { disposition? } \\
\text { No change. }\end{array}$ \\
\hline 79 & $\begin{array}{l}\text { Process sampling } \\
\text { strategy for Material } \\
\text { Control and Quality. }\end{array}$ & & & & & & & & & & & $\mathrm{X}$ & No change. \\
\hline 80 & $\begin{array}{l}\text { Cold Chemical } \\
\text { Storage controls for } \\
\text { shelf life concerns. }\end{array}$ & & & & & & & & & & & & $\begin{array}{l}\text { File } \\
\text { No change. }\end{array}$ \\
\hline 81 & $\begin{array}{l}\text { Tank capacity } \\
\text { requirements to } \\
\text { support "Drainback" } \\
\text { concerns. }\end{array}$ & & & & & & & & & & & $X$ & No change. \\
\hline
\end{tabular}




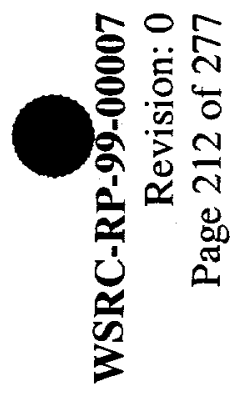

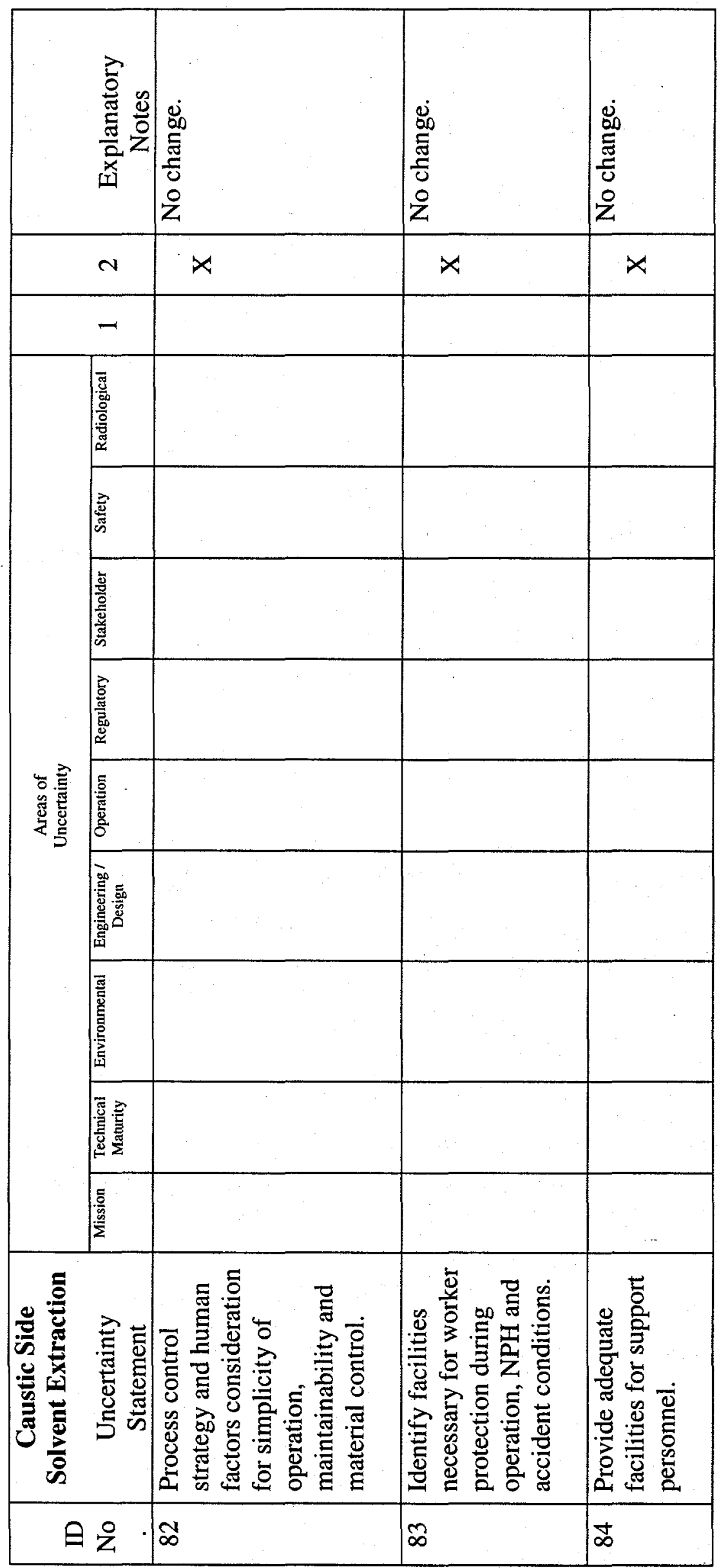




\subsubsection{CST Non-Elutable Ion Exchange}

\begin{tabular}{|c|c|c|c|c|c|c|c|c|c|c|c|c|c|}
\hline \multirow{2}{*}{$\begin{array}{l}\text { ID } \\
\text { No }\end{array}$} & \multirow{2}{*}{$\begin{array}{l}\text { CST Non- } \\
\text { Elutable Ion } \\
\text { Exchange } \\
\text { Uncertainty } \\
\text { Statement }\end{array}$} & \multicolumn{9}{|c|}{$\begin{array}{l}\text { Areas of } \\
\text { Uncertainty }\end{array}$} & \multirow[b]{2}{*}{1} & \multirow[b]{2}{*}{2} & \multirow[b]{2}{*}{ Explanatory Notes } \\
\hline & & Mission & $\begin{array}{l}\text { Technical } \\
\text { Maturity }\end{array}$ & Environmental & $\begin{array}{c}\text { Engineering / } \\
\text { Design }\end{array}$ & Operation & Regulatory & Stakeholder & Safety & Radiological & & & \\
\hline 1 & $\begin{array}{l}\text { What happens to } \\
\text { the resin and } \\
\text { cesium in a loaded } \\
\text { column under } \\
\text { accident } \\
\text { conditions? }\end{array}$ & & & & & & & & & & & $\mathrm{X}$ & No change. \\
\hline 2 & $\begin{array}{l}\text { The neptunium } \\
\text { content in certain } \\
\text { HLW Tanks may } \\
\text { impact the PA and } \\
\text { WAC. }\end{array}$ & & & & & & & & & & & & $\begin{array}{l}\text { File } \\
\text { No change. }\end{array}$ \\
\hline 3 & $\begin{array}{l}\text { What is the fate of } \\
\text { Am in the process? }\end{array}$ & & $\mathrm{x}$ & & & & & & & & $X$ & & $\begin{array}{l}\text { Does MST strike } \\
\text { affect Am } \\
\text { disposition? } \\
\text { No change. }\end{array}$ \\
\hline 4 & $\begin{array}{l}\text { Can not sample } \\
\text { and analyze } \\
\text { composition of } \\
\text { CST in } \\
\text { conjunction with } \\
\text { other DWPF feed } \\
\text { components? }\end{array}$ & & & & & & & & & & & $\mathrm{X}$ & No change. \\
\hline
\end{tabular}


Page 214 of 277

\begin{tabular}{|c|c|c|c|c|c|c|c|c|c|c|c|c|c|}
\hline \multirow{2}{*}{$\begin{array}{l}\text { ID } \\
\text { No }\end{array}$} & \multirow{2}{*}{$\begin{array}{c}\text { CST Non- } \\
\text { Elutable Ion } \\
\text { Exchange } \\
\text { Uncertainty } \\
\text { Statement } \\
\end{array}$} & \multicolumn{9}{|c|}{$\begin{array}{l}\text { Areas of } \\
\text { Uncertainty }\end{array}$} & \multirow[b]{2}{*}{1} & \multirow[b]{2}{*}{2} & \multirow[b]{2}{*}{ Explanatory Notes } \\
\hline & & Mission & $\begin{array}{l}\text { Technical } \\
\text { Maturity }\end{array}$ & Environmental & $\begin{array}{l}\text { Engineering / } \\
\text { Design }\end{array}$ & Operation & Regulatory & Stakeholder & Safety & $\begin{array}{l}\text { Radiological } \\
\end{array}$ & & & \\
\hline 5 & $\begin{array}{l}\text { Can not meet the } \\
\text { glass composition } \\
\text { EA standards and } \\
\text { processing limit? }\end{array}$ & & $\mathrm{x}$ & & & & & & & & $\mathrm{X}$ & & $\begin{array}{l}\text { R\&D results } \\
\text { indicate CST glass } \\
\text { is durable and not } \\
\text { predictable. } \\
\text { WSRC-TR-99- } \\
00245\end{array}$ \\
\hline 6 & $\begin{array}{l}\text { Can homogeneity } \\
\text { be maintained in } \\
\text { the slurry, in } \\
\text { particle size, in } \\
\text { sampling, and } \\
\text { transfer? }\end{array}$ & & & & $\mathrm{x}$ & & & & & & $\mathrm{X}$ & & $\begin{array}{l}\text { R\&D results } \\
\text { indicate } \\
\text { hydraguard } \\
\text { sampling } \\
\text { modifications are } \\
\text { required. } \\
\text { WSRC-RP-99- } \\
00232\end{array}$ \\
\hline 7 & $\begin{array}{l}\text { Can we satisfy test } \\
\text { requirements with } \\
\text { limited access to } \\
\text { DWPF? }\end{array}$ & : & & & & & & & & & & $X$ & No change. \\
\hline 8 & $\begin{array}{l}\text { More variables to } \\
\text { control and the } \\
\text { impact on the } \\
\text { process. (MST } \\
\text { and Sludge, CST, } \\
\text { Sludge, Frit). }\end{array}$ & & & & & & & & & & & $X$ & No change. \\
\hline
\end{tabular}




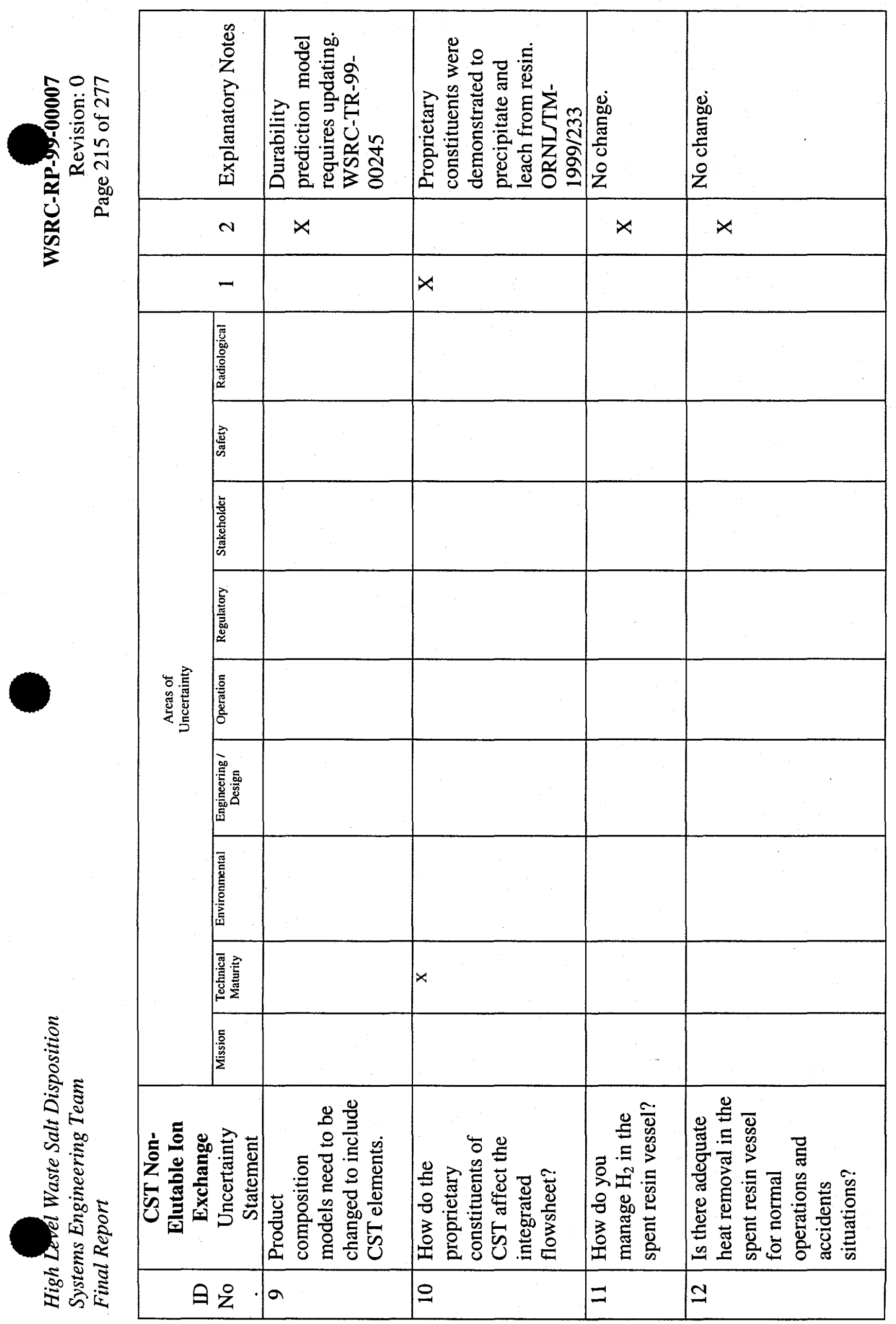




\begin{tabular}{|c|c|c|c|c|c|c|c|c|c|c|c|c|c|}
\hline \multirow{2}{*}{$\begin{array}{l}\text { ID } \\
\text { No }\end{array}$} & \multirow{2}{*}{$\begin{array}{c}\text { CST Non- } \\
\text { Elutable Ion } \\
\text { Exchange } \\
\text { Uncertainty } \\
\text { Statement } \\
\end{array}$} & \multicolumn{9}{|c|}{$\begin{array}{l}\text { Areas of } \\
\text { Uncertainty }\end{array}$} & \multirow[b]{2}{*}{1} & \multirow[b]{2}{*}{2} & \multirow[b]{2}{*}{ Explanatory Notes } \\
\hline & & Mission & $\begin{array}{l}\text { Technical } \\
\text { Maturity }\end{array}$ & Environmental & $\begin{array}{c}\text { Engineering / } \\
\text { Design }\end{array}$ & Operation & Regulatory & Stakeholder & Safety & $\overline{\text { Radiological }}$ & & & \\
\hline 13 & $\begin{array}{l}\text { Control of spent } \\
\text { CST resin spills } \\
\text { and material } \\
\text { recovery. }\end{array}$ & & & & & & & & & & & $\mathrm{X}$ & No change. \\
\hline 14 & $\begin{array}{l}\text { How can you keep } \\
\text { your sluice line } \\
\text { from plugging? }\end{array}$ & & & & & & & & & & & $\mathrm{X}$ & No change. \\
\hline 15 & $\begin{array}{l}\text { Inability to remove } \\
\text { spent resin from a } \\
\text { column. }\end{array}$ & $\vdots$ & & & & & & & & $\mathrm{x}$ & $X$ & & $\begin{array}{l}\text { Need contingency } \\
\text { for unloading resin } \\
\text { from a column that } \\
\text { is plugged. R\&D } \\
\text { demonstrated } \\
\text { normal column } \\
\text { resin unloading. } \\
\text { ORNL/TM- } \\
\text { 1999/103 }\end{array}$ \\
\hline 16 & $\begin{array}{l}\text { Slurry (loaded } \\
\text { CST) } \\
\text { abrasion/erosion } \\
\text { problems on coils } \\
\text { and agitators, } \\
\text { pumps \& valves. }\end{array}$ & & & & & & & & & & & $X$ & No change. \\
\hline
\end{tabular}




\begin{tabular}{|c|c|c|c|c|c|}
\hline \multirow[t]{2}{*}{ 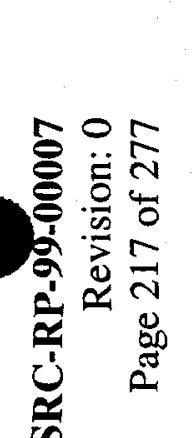 } & 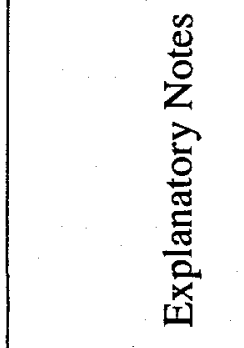 & 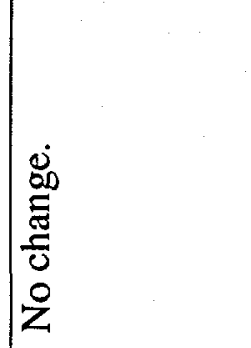 & 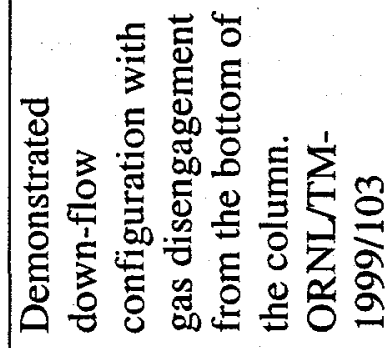 & 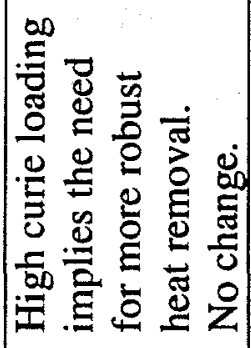 & 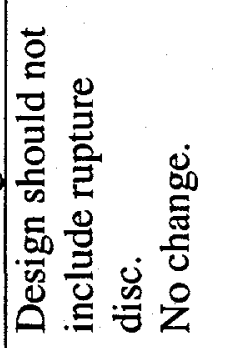 \\
\hline & $N$ & $x$ & $x$ & & $x$ \\
\hline & - & & & $x$ & \\
\hline & 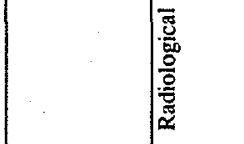 & & & & \\
\hline & 童 & & & & \\
\hline & 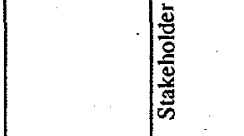 & & & & \\
\hline & $\mid$ & & & & \\
\hline & 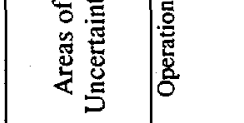 & & & & \\
\hline & 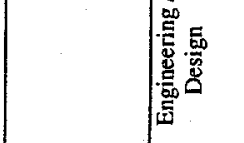 & & 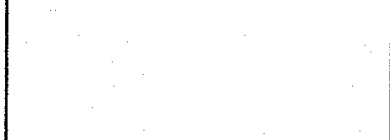 & $x$ & \\
\hline & 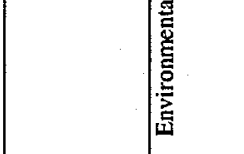 & & & & \\
\hline & 票高 & & & & \\
\hline 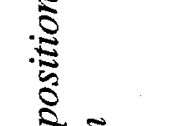 & $\mid \begin{array}{c}5 \\
\frac{5}{W} \\
\frac{0}{2} \\
\end{array}$ & & & - & \\
\hline 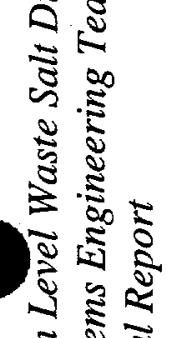 & 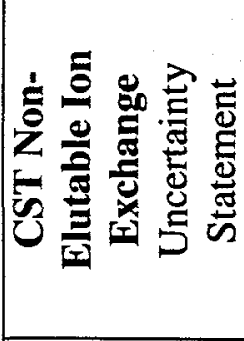 & 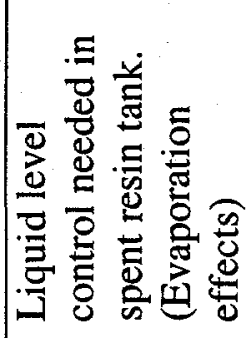 & 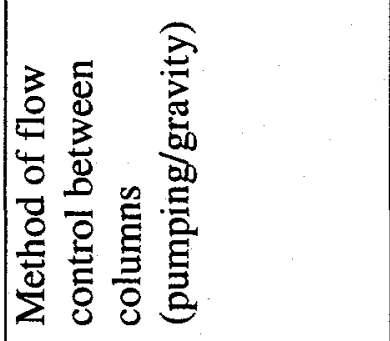 & 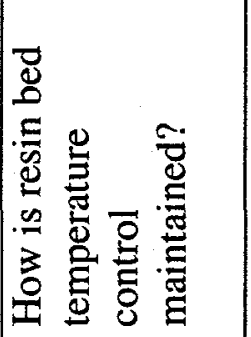 & 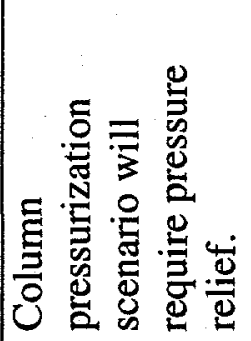 \\
\hline 脑离这 & @ & $\equiv$ & $\infty$ & 은 & 유 \\
\hline
\end{tabular}


Page 218 of 277

\begin{tabular}{|c|c|c|c|c|c|c|c|c|c|c|c|c|c|}
\hline \multirow{2}{*}{$\begin{array}{l}\text { ID } \\
\text { No }\end{array}$} & \multirow{2}{*}{$\begin{array}{c}\text { CST Non- } \\
\text { Elutable Ion } \\
\text { Exchange } \\
\text { Uncertainty } \\
\text { Statement } \\
\end{array}$} & & \multicolumn{5}{|c|}{$\begin{array}{l}\text { Areas of } \\
\text { Uncertainty }\end{array}$} & \multirow[b]{2}{*}{1} & \multirow[b]{2}{*}{2} & \multirow[b]{2}{*}{ Explanatory Notes } \\
\hline & & Mission & $\begin{array}{l}\text { Technical } \\
\text { Maturity }\end{array}$ & Environmental & $\begin{array}{c}\text { Engineering / } \\
\text { Design }\end{array}$ & Operation & Regulatory & Stakeholder & Safety & $\begin{array}{l}\text { Radiological } \\
\end{array}$ & & & \\
\hline 21 & $\begin{array}{l}\text { Corrosion and } \\
\text { pluggage of resin } \\
\text { Johnson screen. }\end{array}$ & & & & & & & & & & & $\mathrm{X}$ & No change. \\
\hline 22 & $\begin{array}{l}\text { Remotability and } \\
\text { replacement of } \\
\text { resin Johnson } \\
\text { screen. }\end{array}$ & & & & & & & & & & & $X$ & No change. \\
\hline 23 & $\begin{array}{l}\text { Filtration method } \\
\text { for fines in high } \\
\text { caustic } \\
\text { environment. }\end{array}$ & & & & & & & & & & & $X$ & No change. \\
\hline 24 & $\begin{array}{l}\text { Adequacy of } \\
\text { monitoring system } \\
\text { to determine when } \\
\text { to change out } \\
\text { columns. }\end{array}$ & 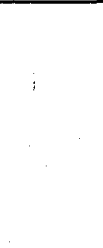 & & & & & & & & & & $X$ & No change. \\
\hline 25 & $\begin{array}{l}\text { Ensure installation } \\
\text { of sample points } \\
\text { after each column } \\
\text { for startup and } \\
\text { operations. }\end{array}$ & & & & & & & & & & & $\mathrm{X}$ & No change. \\
\hline
\end{tabular}


Page 219 of 277

\begin{tabular}{|c|c|c|c|c|c|c|c|c|c|c|c|c|c|}
\hline \multirow{2}{*}{$\begin{array}{l}\text { ID } \\
\text { No }\end{array}$} & \multirow{2}{*}{$\begin{array}{l}\text { CST Non- } \\
\text { Elutable Ion } \\
\text { Exchange } \\
\text { Uncertainty } \\
\text { Statement }\end{array}$} & \multicolumn{9}{|c|}{$\begin{array}{l}\text { Areas of } \\
\text { Uncertainty }\end{array}$} & \multirow[b]{2}{*}{1} & \multirow[b]{2}{*}{2} & \multirow[b]{2}{*}{ Explanatory Notes } \\
\hline & & Mission & $\begin{array}{l}\text { Technical } \\
\text { Maturity }\end{array}$ & Environmental & $\begin{array}{c}\text { Engineering/ } \\
\text { Design }\end{array}$ & Operation & Regulatory & Stakeholder & $\begin{array}{l}\text { Safety } \\
\end{array}$ & Radiological & & & \\
\hline 26 & $\begin{array}{l}\text { Resin blinding } \\
\text { with } \mathrm{H}_{2}, \mathrm{O}_{2} \mathrm{NH}_{3} \\
\text { and steam during } \\
\text { no flow and restart } \\
\text { conditions. }\end{array}$ & & & & & & & & & & & $\mathrm{X}$ & $\begin{array}{l}\text { Need degassing } \\
\text { strategy for the } \\
\text { resin. } \\
\text { No change. }\end{array}$ \\
\hline 27 & $\begin{array}{l}\text { Potential for } \\
\text { channeling in the } \\
\text { column and } \\
\text { remotability of } \\
\text { redistributors. }\end{array}$ & & & & & & & & & & & $X$ & No change. \\
\hline 28 & $\begin{array}{l}\text { Mis-sequencing of } \\
\text { the column feed } \\
\text { carousel during } \\
\text { transition from salt } \\
\text { solution to water. }\end{array}$ & & & & & & & & & & & $\mathrm{X}$ & No change. \\
\hline 29 & $\begin{array}{l}\text { Switching feeds } \\
(\mathrm{pH}) \text { may cause } \\
\text { precipitation ( } \mathrm{AL} \text { ) } \\
\text { in the column. }\end{array}$ & & & & & & & & & & & $X$ & No change. \\
\hline
\end{tabular}




\begin{tabular}{|c|c|c|c|c|c|c|c|c|c|c|c|c|c|}
\hline \multirow{2}{*}{$\begin{array}{l}\text { ID } \\
\text { No }\end{array}$} & \multirow{2}{*}{$\begin{array}{c}\text { CST Non- } \\
\text { Elutable Ion } \\
\text { Exchange } \\
\text { Uncertainty } \\
\text { Statement } \\
\end{array}$} & \multicolumn{9}{|c|}{$\begin{array}{l}\text { Areas of } \\
\text { Uncertainty }\end{array}$} & \multirow[b]{2}{*}{1} & \multirow[b]{2}{*}{2} & \multirow[b]{2}{*}{ Explanatory Notes } \\
\hline & & Mission & $\begin{array}{l}\text { Technical } \\
\text { Maturity }\end{array}$ & Environmental & $\begin{array}{l}\text { Engineering / } \\
\text { Design }\end{array}$ & Operation & Regulatory & Stakeholder & Safety & Radiological & & & \\
\hline 30 & $\begin{array}{l}\text { Temperature } \\
\text { changes or } \\
\text { chemistry changes } \\
\text { may cause post } \\
\text { precipitation after } \\
\text { the MST strike. }\end{array}$ & & $\mathrm{X}$ & & & & & & & & $\mathrm{X}$ & & $\begin{array}{l}\text { Thermodynamic } \\
\text { calculations } \\
\text { indicate aluminum } \\
\text { and silica } \\
\text { precipitation is } \\
\text { probable. } \\
\text { HLW-SDT-99- } \\
0303\end{array}$ \\
\hline 31 & $\begin{array}{l}\text { Dumping the resin } \\
\text { out the bottom of } \\
\text { the column will } \\
\text { hurt the DF after } \\
\text { adding fresh resin } \\
\text { if residue is left } \\
\text { behind. }\end{array}$ & & & & & & & & & & & & $\begin{array}{l}\text { File } \\
\text { No change. }\end{array}$ \\
\hline 32 & $\begin{array}{l}\text { Potential for } \\
\text { breakthrough and } \\
\text { transfer of solids } \\
\text { to columns from } \\
\text { MST Strike. }\end{array}$ & & & & & & & & & & & $\mathrm{X}$ & No change. \\
\hline 33 & $\begin{array}{l}\text { Hydrogen control } \\
\text { in the MST strike } \\
\text { process. }\end{array}$ & & & & & & & & & & & $\mathrm{X}$ & No change. \\
\hline
\end{tabular}


Revision: 0

Page 221 of 277

\begin{tabular}{|c|c|c|c|c|c|c|c|c|c|c|c|c|c|}
\hline \multirow{2}{*}{$\begin{array}{l}\text { ID } \\
\text { No }\end{array}$} & \multirow{2}{*}{$\begin{array}{c}\text { CST Non- } \\
\text { Elutable Ion } \\
\text { Exchange } \\
\text { Uncertainty } \\
\text { Statement } \\
\end{array}$} & \multicolumn{9}{|c|}{$\begin{array}{l}\text { Areas of } \\
\text { Uncertainty }\end{array}$} & \multirow[b]{2}{*}{1} & \multirow[b]{2}{*}{2} & \multirow[b]{2}{*}{ Explanatory Notes } \\
\hline & & Mission & $\begin{array}{l}\text { Technical } \\
\text { Maturity }\end{array}$ & Environmental & $\begin{array}{c}\text { Engineering/ } \\
\text { Design }\end{array}$ & Operation & Regulatory & Stakeholder & Safety & Radiological & & & \\
\hline 34 & $\begin{array}{l}\text { Analysis delay (1 } \\
\text { week) in } \\
\text { measuring for } S_{r} \\
\text { DF in MST } \\
\text { process. }\end{array}$ & & $\mathrm{x}$ & & & & & & & & $X$ & & $\begin{array}{l}\text { Requires new } \\
\text { analytical } \\
\text { techniques. } \\
\text { No change. }\end{array}$ \\
\hline 35 & $\begin{array}{l}\text { Can the spent resin } \\
\text { be converted from } \\
\text { granular } \\
\text { engineered form to } \\
\text { fine powder easily } \\
\text { with mixing and } \\
\text { high shear? This } \\
\text { can improve } \\
\text { transfer, sampling, } \\
\text { and homogeneity. }\end{array}$ & & & & . & & & & * & & & $\mathrm{X}$ & No change. \\
\hline 36 & $\begin{array}{l}\text { Can pressure } \\
\text { gradients crush the } \\
\text { resin during } \\
\text { column } \\
\text { operations? }\end{array}$ & & & & $\mathrm{x}$ & & & & & & $\mathrm{X}$ & & $\begin{array}{l}\text { May generate } \\
\text { excess fines and } \\
\text { reduce filter } \\
\text { efficiency. } \\
\text { No change. }\end{array}$ \\
\hline 37 & $\begin{array}{l}\text { Contingency to } \\
\text { replace CST with } \\
\text { elutable resin. }\end{array}$ & & & & & & & & & & & & $\begin{array}{l}\text { File } \\
\text { No change. }\end{array}$ \\
\hline
\end{tabular}


Page 222 of 277

\begin{tabular}{|c|c|c|c|c|c|c|c|c|c|c|c|c|c|}
\hline \multirow{2}{*}{$\begin{array}{l}\text { ID } \\
\text { No }\end{array}$} & \multirow{2}{*}{$\begin{array}{l}\text { CST Non- } \\
\text { Elutable Ion } \\
\text { Exchange } \\
\text { Uncertainty } \\
\text { Statement } \\
\end{array}$} & \multicolumn{9}{|c|}{$\begin{array}{l}\text { Areas of } \\
\text { Uncertainty }\end{array}$} & \multirow[b]{2}{*}{1} & \multirow[b]{2}{*}{2} & \multirow[b]{2}{*}{ Explanatory Notes } \\
\hline & & Mission & $\begin{array}{l}\text { Technical } \\
\text { Maturity }\end{array}$ & Environmental & $\begin{array}{c}\text { Engineering / } \\
\text { Design }\end{array}$ & Operation & Regulatory & Stakeholder & Safety & Radiological & & & \\
\hline 38 & $\begin{array}{l}\text { Application of } \\
\text { carousel design in } \\
\text { a remote } \\
\text { environment (eg } \\
\text { operation and } \\
\text { maintenance of } \\
\text { valves). }\end{array}$ & & & & $\mathrm{x}$ & & & & & & $X$ & & $\begin{array}{l}\text { Hanford has } \\
\text { experienced major } \\
\text { problems. Multiple } \\
\text { column } \\
\text { connections and } \\
\text { valve concerns. } \\
\text { No change. }\end{array}$ \\
\hline 39 & $\begin{array}{l}\text { Temperature } \\
\text { monitoring in } \\
\text { column can be } \\
\text { used for loading } \\
\text { profile }\end{array}$ & & & & & & & & & & & $\mathrm{X}$ & No change. \\
\hline 40 & $\begin{array}{l}\text { Fate and } \\
\text { downstream } \\
\text { impact of oxalate, } \\
\text { after a cross flow } \\
\text { filter cleaning } \\
\text { operation }\end{array}$ & i & & & & & & & & & & & $\begin{array}{l}\text { File } \\
\text { No change. }\end{array}$ \\
\hline 41 & $\begin{array}{l}\text { Rate of spent } \\
\text { equipment } \\
\text { generation and its } \\
\text { disposal. }\end{array}$ & & & & & & & & & & & $X$ & No change. \\
\hline
\end{tabular}


Page 223 of 277

\begin{tabular}{|c|c|c|c|c|c|c|c|c|c|c|c|c|c|}
\hline \multirow{2}{*}{$\begin{array}{l}\text { D } \\
\text { No }\end{array}$} & \multirow{2}{*}{$\begin{array}{l}\text { CST Non- } \\
\text { Elutable Ion } \\
\text { Exchange } \\
\text { Uncertainty } \\
\text { Statement }\end{array}$} & \multicolumn{9}{|c|}{$\begin{array}{l}\text { Areas of } \\
\text { Uncertainty }\end{array}$} & \multirow[b]{2}{*}{1} & \multirow[b]{2}{*}{2} & \multirow[b]{2}{*}{ Explanatory Notes } \\
\hline & & Mission & $\begin{array}{l}\text { Technical } \\
\text { Maturity }\end{array}$ & Environmental & $\begin{array}{c}\text { Engineering/ } \\
\text { Design }\end{array}$ & Operation & Regulatory & Stakeholder & Safety & Radiological & & & \\
\hline 42 & $\begin{array}{l}\text { Deconning of } \\
\text { equipment may be } \\
\text { difficult because of } \\
\text { fines. }\end{array}$ & & & & & & & & & & & $\mathrm{X}$ & No change. \\
\hline 43 & $\begin{array}{l}\text { Fines } \\
\text { accumulation of } \\
\text { loaded CST in } \\
\text { process ventilation } \\
\text { system results in } \\
\text { very hot system. }\end{array}$ & & & & & & & & & & & $\mathrm{X}$ & No change. \\
\hline 44 & $\begin{array}{l}\text { Is the shielding in } \\
\text { the current transfer } \\
\text { lines adequate for } \\
\text { transferring spent/ } \\
\text { loaded resin? }\end{array}$ & & & & & & & & & $\mathrm{x}$ & $\mathrm{X}$ & & $\begin{array}{l}\text { Current transfer } \\
\text { lines designed for } \\
40 \text { curies/gallon } \\
\text { versus potentially } \\
500 \text { curies/gallon. } \\
\text { No change. }\end{array}$ \\
\hline 45 & $\begin{array}{l}\text { How do you } \\
\text { manage large curie } \\
\text { inventories in } \\
\text { facilities? }\end{array}$ & & & & & & & & & & & & $\begin{array}{l}\text { File } \\
\text { No change. }\end{array}$ \\
\hline 46 & $\begin{array}{l}\text { Failure of seals, } \\
\text { elastomers, } \\
\text { etc...from high rad } \\
\text { field. }\end{array}$ & & & & & & & & & & & $\mathrm{X}$ & No change. \\
\hline
\end{tabular}


Revision: 0

Page 224 of 277

\begin{tabular}{|c|c|c|c|c|c|c|c|c|c|c|c|c|c|}
\hline \multirow{2}{*}{$\begin{array}{l}\text { ID } \\
\text { No }\end{array}$} & \multirow{2}{*}{$\begin{array}{c}\text { CST Non- } \\
\text { Elutable Ion } \\
\text { Exchange } \\
\text { Uncertainty } \\
\text { Statement } \\
\end{array}$} & \multicolumn{9}{|c|}{$\begin{array}{l}\text { Areas of } \\
\text { Uncertainty }\end{array}$} & \multirow[b]{2}{*}{1} & \multirow[b]{2}{*}{2} & \multirow{2}{*}{ Explanatory Notes } \\
\hline & & Mission & $\begin{array}{l}\text { Technical } \\
\text { Maturity }\end{array}$ & Environmental & $\begin{array}{c}\text { Engineering / } \\
\text { Design }\end{array}$ & Operation & Regulatory & Stakeholder & Safety & Radiological & & & \\
\hline 47 & $\begin{array}{l}\text { Need for cell-to- } \\
\text { clean-area } \\
\text { isolation: } \\
\text { (eg. Impulse lines, } \\
\text { transfer pumps) }\end{array}$ & & & & & & & & & & & $X$ & No change. \\
\hline 48 & $\begin{array}{l}\text { Disposal method } \\
\text { for clean CST } \\
\text { fines. }\end{array}$ & & & & & & & & & & & $\mathrm{X}$ & No change. \\
\hline 49 & $\begin{array}{l}\text { Impact of SME } \\
\text { and SRAT carry- } \\
\text { over of CST fines } \\
\text { DWPF recycle. }\end{array}$ & & & & & & & & & & & $X$ & $\begin{array}{l}\text { Evaporator } \\
\text { installed in DWPF } \\
\text { would mitigate the } \\
\text { recycle concern. } \\
\text { No change. } \\
\text { WSRC-RP-99- } \\
00005\end{array}$ \\
\hline 50 & $\begin{array}{l}\text { Minimize spark } \\
\text { sources in } \mathrm{H}_{2} \text { rich } \\
\text { areas. }\end{array}$ & & & & & & & & & & & $\mathrm{X}$ & No change. \\
\hline 51 & $\begin{array}{l}\text { Monitoring GT-73 } \\
\text { performance and } \\
\text { breakthrough. }\end{array}$ & & & & & & & & & & & & File \\
\hline
\end{tabular}




\begin{tabular}{|c|c|c|c|c|c|c|c|c|c|c|c|c|c|}
\hline \multirow{2}{*}{$\begin{array}{l}\text { ID } \\
\text { No }\end{array}$} & \multirow{2}{*}{$\begin{array}{c}\text { CST Non- } \\
\text { Elutable Ion } \\
\text { Exchange } \\
\text { Uncertainty } \\
\text { Statement } \\
\end{array}$} & \multicolumn{9}{|c|}{$\begin{array}{l}\text { Areas of } \\
\text { Uncertainty }\end{array}$} & \multirow[b]{2}{*}{1} & \multirow[b]{2}{*}{2} & \multirow[b]{2}{*}{ Explanatory Notes } \\
\hline & & Mission & $\begin{array}{l}\text { Technical } \\
\text { Maturity }\end{array}$ & Environmental & $\begin{array}{c}\text { Engineering/ } \\
\text { Design }\end{array}$ & Operation & Regulatory & Stakeholder & $\begin{array}{l}\text { Safety } \\
\end{array}$ & Radiological & & & \\
\hline 52 & $\begin{array}{l}\text { Difficulty in } \\
\text { maintaining } \\
\text { remote safety class } \\
\text { pressure relief } \\
\text { valves. }\end{array}$ & & & & & & & & & & & $\mathrm{X}$ & No change. \\
\hline 53 & $\begin{array}{l}\text { Level of functional } \\
\text { testing required. }\end{array}$ & & & & & & & & & & & $\mathrm{X}$ & No change. \\
\hline 54 & $\begin{array}{l}\text { How to test a } \\
\text { freshly filled } \\
\text { column prior to } \\
\text { feeding waste. } \\
\text { (mode switching). }\end{array}$ & & & & & & & & & & & $\mathrm{X}$ & No change. \\
\hline 55 & $\begin{array}{l}\text { TRU } \\
\text { decontamination } \\
\text { may require } \\
\text { excessive MST. }\end{array}$ & 1 & $\mathrm{X}$ & & & & & & & & $X$ & & $\begin{array}{l}\text { Equilibrium } \\
\text { capacity shown to } \\
\text { be sufficient by } \\
\text { R\&D. Neptunium } \\
\text { for Tanks } 33 \text { and } \\
34 \text { will require } \\
\text { blending. } \\
\text { WSRC-TR-99- } \\
\text { 00219 } \\
\text { WSRC-TR-99- } \\
\text { 00286 }\end{array}$ \\
\hline
\end{tabular}




\begin{tabular}{|c|c|c|c|c|c|c|c|c|c|c|c|c|c|}
\hline \multirow{2}{*}{$\begin{array}{l}\text { ID } \\
\text { No }\end{array}$} & \multirow{2}{*}{$\begin{array}{l}\text { CST Non- } \\
\text { Elutable Ion } \\
\text { Exchange } \\
\text { Uncertainty } \\
\text { Statement } \\
\end{array}$} & \multicolumn{9}{|c|}{$\begin{array}{l}\text { Areas of } \\
\text { Uncertainty }\end{array}$} & \multirow[b]{2}{*}{1} & \multirow[b]{2}{*}{2} & \multirow[b]{2}{*}{ Explanatory Notes } \\
\hline & & Mission & $\begin{array}{l}\text { Technical } \\
\text { Maturity }\end{array}$ & Environmental & $\begin{array}{l}\text { Engineering/ } \\
\text { Design }\end{array}$ & Operation & Regulatory & Stakeholder & $\begin{array}{l}\text { Safety } \\
\end{array}$ & $\begin{array}{l}\text { Radiological } \\
\end{array}$ & & & \\
\hline 56 & $\begin{array}{l}\text { Catalytic } \mathrm{H} 2 \\
\text { production from } \\
\text { Formic Acid in } \\
\text { DWPF greater } \\
\text { than Authorization } \\
\text { Basis (AB) } \\
\text { assumptions due to } \\
\text { CST. }\end{array}$ & & - & & & & & & & & & $X$ & $\begin{array}{l}\mathrm{R} \& \mathrm{D} \text { results } \\
\text { indicate } \mathrm{H}_{2} \\
\text { generation rate less } \\
\text { than } \mathrm{AB} \\
\text { assumptions. } \\
\text { WSRC-TR-99- } \\
00302\end{array}$ \\
\hline 57 & $\begin{array}{l}\text { The process } \\
\text { technology for the } \\
\text { SRS application of } \\
\text { CST is not } \\
\text { demonstrated; } \\
\text { therefore, the } \\
\text { design may not } \\
\text { meet performance } \\
\text { requirements. }\end{array}$ & $\therefore$ & $\mathrm{x}$ & & & & & & & & $X$ & & $\begin{array}{l}\text { Pilot } \\
\text { demonstration } \\
\text { needed. } \\
\text { No change. }\end{array}$ \\
\hline 58 & $\begin{array}{l}\text { MST/CST ( } 10 \%) \\
\text { will have } \\
\text { deleterious effects } \\
\text { on the glass form } \\
\text { due to } \\
\text { precipitation of } \\
\text { TiO2 in glass. }\end{array}$ & & & & & & & & & & & & $\begin{array}{l}\text { File } \\
\text { No change. }\end{array}$ \\
\hline
\end{tabular}




\begin{tabular}{|c|c|c|c|c|c|c|c|c|c|c|c|c|c|}
\hline \multirow{2}{*}{$\begin{array}{l}\text { ID } \\
\text { No }\end{array}$} & \multirow{2}{*}{$\begin{array}{c}\text { CST Non- } \\
\text { Elutable Ion } \\
\text { Exchange } \\
\text { Uncertainty } \\
\text { Statement }\end{array}$} & \multicolumn{9}{|c|}{$\begin{array}{l}\text { Areas of } \\
\text { Uncertainty }\end{array}$} & \multirow[b]{2}{*}{1} & \multirow[b]{2}{*}{2} & \multirow[b]{2}{*}{ Explanatory Notes } \\
\hline & & Mission & $\begin{array}{l}\text { Technical } \\
\text { Maturity }\end{array}$ & Environmental & $\begin{array}{c}\text { Engineering / } \\
\text { Design }\end{array}$ & Operation & $\begin{array}{l}\text { Regulatory } \\
\end{array}$ & Stakeholder & Safety & Radiological & & & \\
\hline 59 & $\begin{array}{l}\text { High thermal loads } \\
\text { in columns will } \\
\text { result in } \\
\text { degradation and } \\
\text { unacceptable } \\
\text { column } \\
\text { performance. }\end{array}$ & & & & & & & & & & & $X$ & No change. \\
\hline 60 & $\begin{array}{l}\text { CST cannot be } \\
\text { maintained in a } \\
\text { homogeneous } \\
\text { mixture in SME } \\
\text { Sample and MFT } \\
\text { Feed. }\end{array}$ & & & & & & & & & & & & $\begin{array}{l}\text { File } \\
\text { No change. } \\
\text { WSRC-RP-99- } \\
0232 \\
\text { WSRC-TR-99- } \\
00309\end{array}$ \\
\hline 61 & $\begin{array}{l}\text { Difficulty in } \\
\text { filtration of sludge } \\
\text { and/or MST will } \\
\text { produce low } \\
\text { filtrate flow rates } \\
\text { and require } \\
\text { frequent cleaning }\end{array}$ & 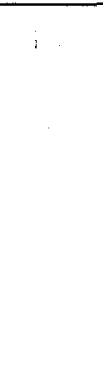 & $x$ & & & - & & & & & $X$ & & $\begin{array}{l}\text { R\&D work has } \\
\text { confirmed this } \\
\text { risk. } \\
\text { WSRC-TR-99- } \\
00346\end{array}$ \\
\hline
\end{tabular}




\begin{tabular}{|c|c|c|c|c|c|c|c|c|c|c|c|c|c|}
\hline \multirow{2}{*}{$\begin{array}{l}\text { ID } \\
\text { No }\end{array}$} & \multirow{2}{*}{$\begin{array}{c}\text { CST Non- } \\
\text { Elutable Ion } \\
\text { Exchange } \\
\text { Uncertainty } \\
\text { Statement } \\
\end{array}$} & \multicolumn{9}{|c|}{$\begin{array}{l}\text { Areas of } \\
\text { Uncertainty }\end{array}$} & \multirow[b]{2}{*}{1} & \multirow[b]{2}{*}{2} & \multirow[b]{2}{*}{ Explanatory Notes } \\
\hline & & Mission & $\begin{array}{l}\text { Technical } \\
\text { Maturity }\end{array}$ & Environmental & $\begin{array}{c}\text { Engineering/ } \\
\text { Design }\end{array}$ & Operation & Regulatory & Stakeholder & Safety & Radiological & & & \\
\hline 62 & $\begin{array}{l}\text { Inability to transfer } \\
\text { the CST slurry in a } \\
\text { controlled manner }\end{array}$ & & & & $\mathrm{x}$ & & & & & & $X$ & & $\begin{array}{l}\text { In preparation for } \\
\text { the hydrogen } \\
\text { generation test size } \\
\text { reduced CST was } \\
\text { observed to pack } \\
\text { and not resuspend. } \\
\text { WSRC-TR-99- } \\
00302\end{array}$ \\
\hline 63 & $\begin{array}{l}\text { Difficulty in } \\
\text { resuspending MST } \\
\text { after long } \\
\text { quiescent period }\end{array}$ & & & & $\bar{x}$ & & & & & & $\mathrm{X}$ & & $\begin{array}{l}\text { After } 60 \text { days of } \\
\text { settling, } \\
\text { resuspension was } \\
\text { not achieved with } \\
\text { proposed } \\
\text { flowsheet design. } \\
\text { ORNL-TM- } \\
\text { 1999/166 }\end{array}$ \\
\hline 64 & $\begin{array}{l}\text { HLW cannot } \\
\text { support tank } \\
\text { blending strategies } \\
\text { for Cs (or other } \\
\text { species) to } \\
\text { support process } \\
\text { requirements. }\end{array}$ & & & & & & & & & & & $\mathrm{X}$ & No change. \\
\hline 65 & $\begin{array}{l}\text { Get CST in recycle } \\
\text { stream from } \\
\text { DWPF }\end{array}$ & & & & & & & & & & & & $\begin{array}{l}\text { File } \\
\text { No change. } \\
\text { WSRC-RP-99- } \\
00005\end{array}$ \\
\hline
\end{tabular}




\begin{tabular}{|c|c|c|c|c|c|c|c|c|c|c|c|c|c|}
\hline \multirow{2}{*}{$\underset{\text { No }}{\text { ID }}$} & \multirow{2}{*}{$\begin{array}{c}\text { CST Non- } \\
\text { Elutable Ion } \\
\text { Exchange } \\
\text { Uncertainty } \\
\text { Statement }\end{array}$} & \multicolumn{9}{|c|}{$\begin{array}{l}\text { Areas of } \\
\text { Uncertainty }\end{array}$} & \multirow[b]{2}{*}{1} & \multirow[b]{2}{*}{2} & \multirow[b]{2}{*}{ Explanatory Notes } \\
\hline & & Mission & $\begin{array}{l}\text { Technical } \\
\text { Maturity }\end{array}$ & Environmental & $\begin{array}{c}\text { Engineering/ } \\
\text { Design }\end{array}$ & Operation & Regulatory & Stakeholder & Safety & $\begin{array}{l}\text { Radiological } \\
\end{array}$ & & & \\
\hline 66 & $\begin{array}{l}\text { GT73 resin will be } \\
\text { selective to Co60 } \\
\text { or other trace } \\
\text { radioisotopes. }\end{array}$ & & & & & & & & & & & & File \\
\hline 67 & $\begin{array}{l}\text { Failure to use } \\
\text { MST on the front } \\
\text { end will lead to } \\
\text { criticality on CST. }\end{array}$ & & & & & & & & & & & & $\begin{array}{l}\text { File } \\
\text { No change. }\end{array}$ \\
\hline 68 & $\begin{array}{l}\text { Deflagration of } \\
\text { resin column due } \\
\text { to radiolysis of } \\
\text { water ( } \mathrm{H} 2 \\
\text { generation). }\end{array}$ & & & & & & & & & & & & $\begin{array}{l}\text { File } \\
\text { No change. }\end{array}$ \\
\hline 69 & $\begin{array}{l}\text { Steam } \\
\text { pressurization of } \\
\text { resin column }\end{array}$ & : & & & & & & & & & & & $\begin{array}{l}\text { File } \\
\text { R\&D results } \\
\text { indicate } \mathrm{H}_{2} \\
\text { generation rate is } \\
\text { less than } \mathrm{AB} \\
\text { assumptions. } \\
\text { No change. }\end{array}$ \\
\hline 70 & $\begin{array}{l}\text { Catalytic } \mathrm{H} 2 \\
\text { production in } \\
\text { DWPF greater } \\
\text { than Authorization } \\
\text { Basis due to CST. }\end{array}$ & & & & & & & & & & & & $\begin{array}{l}\text { File } \\
\text { WSRC-TR-99- } \\
00302\end{array}$ \\
\hline
\end{tabular}


Page 230 of 277

\begin{tabular}{|c|c|c|c|c|c|c|c|c|c|c|c|c|c|}
\hline \multirow{2}{*}{$\begin{array}{l}\text { ID } \\
\text { No }\end{array}$} & \multirow{2}{*}{$\begin{array}{l}\text { CST Non- } \\
\text { Elutable Ion } \\
\text { Exchange } \\
\text { Uncertainty } \\
\text { Statement }\end{array}$} & \multicolumn{9}{|c|}{$\begin{array}{l}\text { Areas of } \\
\text { Uncertainty }\end{array}$} & \multirow[b]{2}{*}{1} & \multirow[b]{2}{*}{2} & \multirow[b]{2}{*}{ Explanatory Notes } \\
\hline & & Mission & $\begin{array}{l}\text { Technical } \\
\text { Maturity }\end{array}$ & Environmental & $\begin{array}{l}\text { Engincering / } \\
\text { Design }\end{array}$ & Operation & Regulatory & Stakeholder & Safety & Radiological & & & \\
\hline 71 & $\begin{array}{l}\text { CST will require } \\
\text { "requalification" } \\
\text { of glass form }\end{array}$ & & & & & & & & & & & & $\begin{array}{l}\text { File } \\
\text { WSRC-TR-99- } \\
00245\end{array}$ \\
\hline 72 & $\begin{array}{l}\text { Expensive and } \\
\text { complex material } \\
\text { handling system } \\
\text { for moving CST } \\
\text { slurry. }\end{array}$ & & & & & & & & & & & & $\begin{array}{l}\text { File } \\
\text { No change. }\end{array}$ \\
\hline 73 & $\begin{array}{l}\text { No means to } \\
\text { adequately analyze } \\
\text { CST in DWPF }\end{array}$ & & & & & & & & & & & & $\begin{array}{l}\text { File } \\
\text { No change. }\end{array}$ \\
\hline 74 & $\begin{array}{l}\text { Mercury removal } \\
\text { resin (GT-73) will } \\
\text { not work in high } \\
\text { caustic } \\
\text { environment ( }>2 \\
\text { Molar). }\end{array}$ & : & & . & & & & & & & & & File \\
\hline 75 & $\begin{array}{l}\text { The CST material } \\
\text { may not be } \\
\text { available in } \\
\text { sufficient } \\
\text { quantities to } \\
\text { support the process } \\
\text { ( } 50 \text { tons per year). }\end{array}$ & & & & & $\mathrm{x}$ & & & & & $\mathrm{X}$ & & \\
\hline
\end{tabular}


Page 231 of 277

\begin{tabular}{|c|c|c|c|c|c|c|c|c|c|c|c|c|c|}
\hline \multirow{2}{*}{$\begin{array}{l}\text { ID } \\
\text { No }\end{array}$} & \multirow{2}{*}{$\begin{array}{l}\text { CST Non- } \\
\text { Elutable Ion } \\
\text { Exchange } \\
\text { Uncertainty } \\
\text { Statement }\end{array}$} & \multicolumn{9}{|c|}{$\begin{array}{l}\text { Areas of } \\
\text { Uncertainty }\end{array}$} & \multirow[b]{2}{*}{1} & \multirow[b]{2}{*}{2} & \multirow[b]{2}{*}{ Explanatory Notes } \\
\hline & & Mission & $\begin{array}{l}\text { Technical } \\
\text { Maturity }\end{array}$ & Environmental & $\begin{array}{c}\text { Engineering / } \\
\text { Design }\end{array}$ & Operation & Regulatory & Stakeholder & Safety & Radiological & & & \\
\hline 76 & $\begin{array}{l}\text { Major sample } \\
\text { station } \\
\text { modifications } \\
\text { affecting DWPF } \\
\text { operations. }\end{array}$ & & & & & $\mathrm{x}$ & & & & & $X$ & & \\
\hline 77 & $\begin{array}{l}\text { TRU } \\
\text { decontamination } \\
\text { with MST is not } \\
\text { adequate. }\end{array}$ & & $\mathbf{X}$ & & & & & & & & $X$ & & $\begin{array}{l}\text { Kinetics for } \\
\text { bounding } \\
\text { plutonium is too } \\
\text { slow. } \\
\text { WSRC-TR-99- } \\
00219 \\
\text { WSRC-TR-99- } \\
00286\end{array}$ \\
\hline 78 & $\begin{array}{l}\text { Accumulation of } \\
\text { hydrogen may } \\
\text { occur in vapor } \\
\text { spaces (including } \\
\text { process vessels) } \\
\text { and } \\
\text { deflagrations/deton } \\
\text { ation could occur } \\
\text { if there is a spark } \\
\text { source. }\end{array}$ & $i$ & & & & & & & & & & $X$ & No change. \\
\hline
\end{tabular}


Page 232 of 277

\begin{tabular}{|c|c|c|c|c|c|c|c|c|c|c|c|c|c|}
\hline \multirow{2}{*}{$\begin{array}{l}\text { ID } \\
\text { No }\end{array}$} & \multirow{2}{*}{$\begin{array}{c}\text { CST Non- } \\
\text { Elutable Ion } \\
\text { Exchange } \\
\text { Uncertainty } \\
\text { Statement } \\
\end{array}$} & \multicolumn{9}{|c|}{$\begin{array}{l}\text { Areas of } \\
\text { Uncertainty }\end{array}$} & \multirow[b]{2}{*}{1} & \multirow[b]{2}{*}{2} & \multirow[b]{2}{*}{ Explanatory Notes } \\
\hline & & Mission & $\begin{array}{l}\text { Technical } \\
\text { Maturity }\end{array}$ & Environmental & $\begin{array}{c}\text { Engineering } \\
\text { Design }\end{array}$ & Operation & Regulatory & Stakeholder & Safety & $\begin{array}{l}\text { Radiological } \\
\end{array}$ & & & \\
\hline 79 & $\begin{array}{l}\text { Process sampling } \\
\text { strategy for } \\
\text { Material Control } \\
\text { and Quality. }\end{array}$ & & & & & & & & & & & $\mathrm{X}$ & No change. \\
\hline 80 & $\begin{array}{l}\text { Cold Chemical } \\
\text { Storage controls } \\
\text { for shelf life } \\
\text { concerns. }\end{array}$ & & & & & & & - & & & & & $\begin{array}{l}\text { File } \\
\text { No change. }\end{array}$ \\
\hline 81 & $\begin{array}{l}\text { Tank capacity } \\
\text { requirements to } \\
\text { support } \\
\text { "Drainback" } \\
\text { concerns. }\end{array}$ & & & & & & & & & & & $\mathrm{X}$ & No change. \\
\hline 82 & $\begin{array}{l}\text { Process control } \\
\text { strategy and } \\
\text { human factors } \\
\text { consideration for } \\
\text { simplicity of } \\
\text { operation, } \\
\text { maintainability } \\
\text { and material } \\
\text { control. }\end{array}$ & & & & & & & & & & & $\mathrm{X}$ & No change. \\
\hline
\end{tabular}




\begin{tabular}{|c|c|c|c|c|c|c|c|c|c|c|c|c|c|}
\hline \multirow{2}{*}{$\begin{array}{l}\text { ID } \\
\text { No }\end{array}$} & \multirow{2}{*}{$\begin{array}{l}\text { CST Non- } \\
\text { Elutable Ion } \\
\text { Exchange } \\
\text { Uncertainty } \\
\text { Statement } \\
\end{array}$} & \multicolumn{9}{|c|}{$\begin{array}{l}\text { Areas of } \\
\text { Uncertainty }\end{array}$} & \multirow[b]{2}{*}{1} & \multirow[b]{2}{*}{2} & \multirow[b]{2}{*}{ Explanatory Notes } \\
\hline & & Mission & $\begin{array}{l}\text { Technical } \\
\text { Maturity }\end{array}$ & Environmental & $\begin{array}{c}\text { Engineering / } \\
\text { Design }\end{array}$ & Operation & Regulatory & Stakeholder & Safety & $\overline{R a d i o l o g i c a l}$ & & & \\
\hline 83 & $\begin{array}{l}\text { Identify facilities } \\
\text { necessary for } \\
\text { worker protection } \\
\text { during operation, } \\
\text { NPH and accident } \\
\text { conditions. }\end{array}$ & & & & & & & & & & & $\mathrm{X}$ & No change. \\
\hline 84 & $\begin{array}{l}\text { Provide adequate } \\
\text { facilities for } \\
\text { support personnel. }\end{array}$ & & & & & & & & & & & $\mathrm{X}$ & No change. \\
\hline 85 & $\begin{array}{l}\text { Existing transfer } \\
\text { lines and tank size } \\
\text { and drain back not } \\
\text { adequate, and } \\
\text { leads to } \\
\text { inefficiencies. }\end{array}$ & : & & & & & & & & & & $\mathrm{X}$ & No change. \\
\hline 86 & $\begin{array}{l}\text { Solids on filters } \\
\text { will not dissolve or } \\
\text { would be difficult } \\
\text { to dissolve in } \\
\text { oxalic acid. }\end{array}$ & & & & & & & & & & & & $\begin{array}{l}\text { File } \\
\text { No change. }\end{array}$ \\
\hline
\end{tabular}


Revision: 0

Page 234 of 277

\begin{tabular}{|c|c|c|c|c|c|c|c|c|c|c|c|c|c|}
\hline \multirow{2}{*}{$\begin{array}{l}\text { ID } \\
\text { No }\end{array}$} & \multirow{2}{*}{$\begin{array}{l}\text { CST Non- } \\
\text { Elutable Ion } \\
\text { Exchange } \\
\text { Uncertainty } \\
\text { Statement } \\
\end{array}$} & \multicolumn{9}{|c|}{$\begin{array}{l}\text { Areas of } \\
\text { Uncertainty }\end{array}$} & \multirow[b]{2}{*}{1} & \multirow[b]{2}{*}{2} & \multirow[b]{2}{*}{ Explanatory Notes } \\
\hline & & Mission & $\begin{array}{l}\text { Technical } \\
\text { Maturity }\end{array}$ & Environmental & $\begin{array}{c}\text { Enginecring / } \\
\text { Design }\end{array}$ & Operation & Regulatory & Stakeholder & Safety & Radiological & & & \\
\hline 87 & $\begin{array}{l}\text { Require adequate } \\
\text { size of remote and } \\
\text { contact } \\
\text { decontamination } \\
\text { cells, with } \\
\text { adequate crane } \\
\text { coverage. }\end{array}$ & & & & & & & & & & & $\mathrm{X}$ & No change. \\
\hline 88 & $\begin{array}{l}\text { Need for docking } \\
\text { door to maintain } \\
\text { clean crane } \\
\text { controls and } \\
\text { electronics. }\end{array}$ & & & & & & & & & & & $X$ & No change. \\
\hline 89 & $\begin{array}{l}\text { Adequate process } \\
\text { instrumentation to } \\
\text { detect process } \\
\text { upsets and perform } \\
\text { routine } \\
\text { monitoring. }\end{array}$ & ; & & & & & & & & & & $X$ & No change. \\
\hline
\end{tabular}




\begin{tabular}{|c|c|c|c|c|c|c|c|c|c|c|c|c|c|}
\hline \multirow{2}{*}{$\begin{array}{l}\text { ID } \\
\text { No }\end{array}$} & \multirow{2}{*}{$\begin{array}{l}\text { CST Non- } \\
\text { Elutable Ion } \\
\text { Exchange } \\
\text { Uncertainty } \\
\text { Statement } \\
\end{array}$} & \multicolumn{9}{|c|}{$\begin{array}{c}\text { Areas of } \\
\text { Uncertainty }\end{array}$} & \multirow[b]{2}{*}{1} & \multirow[b]{2}{*}{2} & \multirow[b]{2}{*}{ Explanatory Notes } \\
\hline & & Mission & $\begin{array}{l}\text { Technical } \\
\text { Maturity }\end{array}$ & Environmental & $\begin{array}{l}\text { Engineering / } \\
\text { Design }\end{array}$ & Operation & Regulatory & Stakeholder & $\begin{array}{l}\text { Safety } \\
\end{array}$ & Radiological & & & \\
\hline 90 & $\begin{array}{l}\text { Process chemistry } \\
\text { understanding and } \\
\text { application are still } \\
\text { under } \\
\text { development, } \\
\text { resulting in } 96-1 \\
\text { lessons learned not } \\
\text { yet implemented }\end{array}$ & & $\mathrm{x}$ & & & & & & & & $X$ & & $\begin{array}{l}\text { Product is } \\
\text { considered to be } \\
\text { under development } \\
\text { and by experiment, } \\
\text { has exhibited } \\
\text { stability and } \\
\text { leaching problems. } \\
\text { ORNL/TM- } \\
\text { 1999/233 }\end{array}$ \\
\hline
\end{tabular}



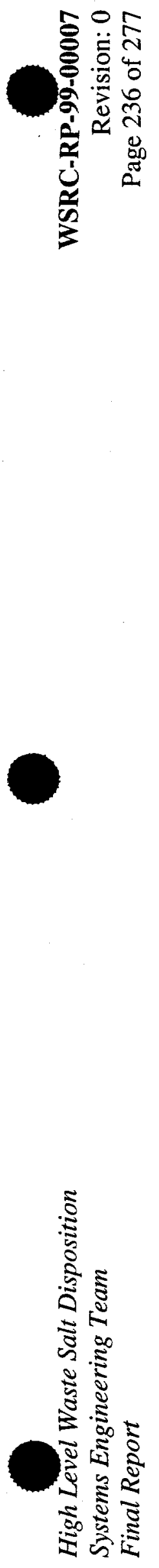

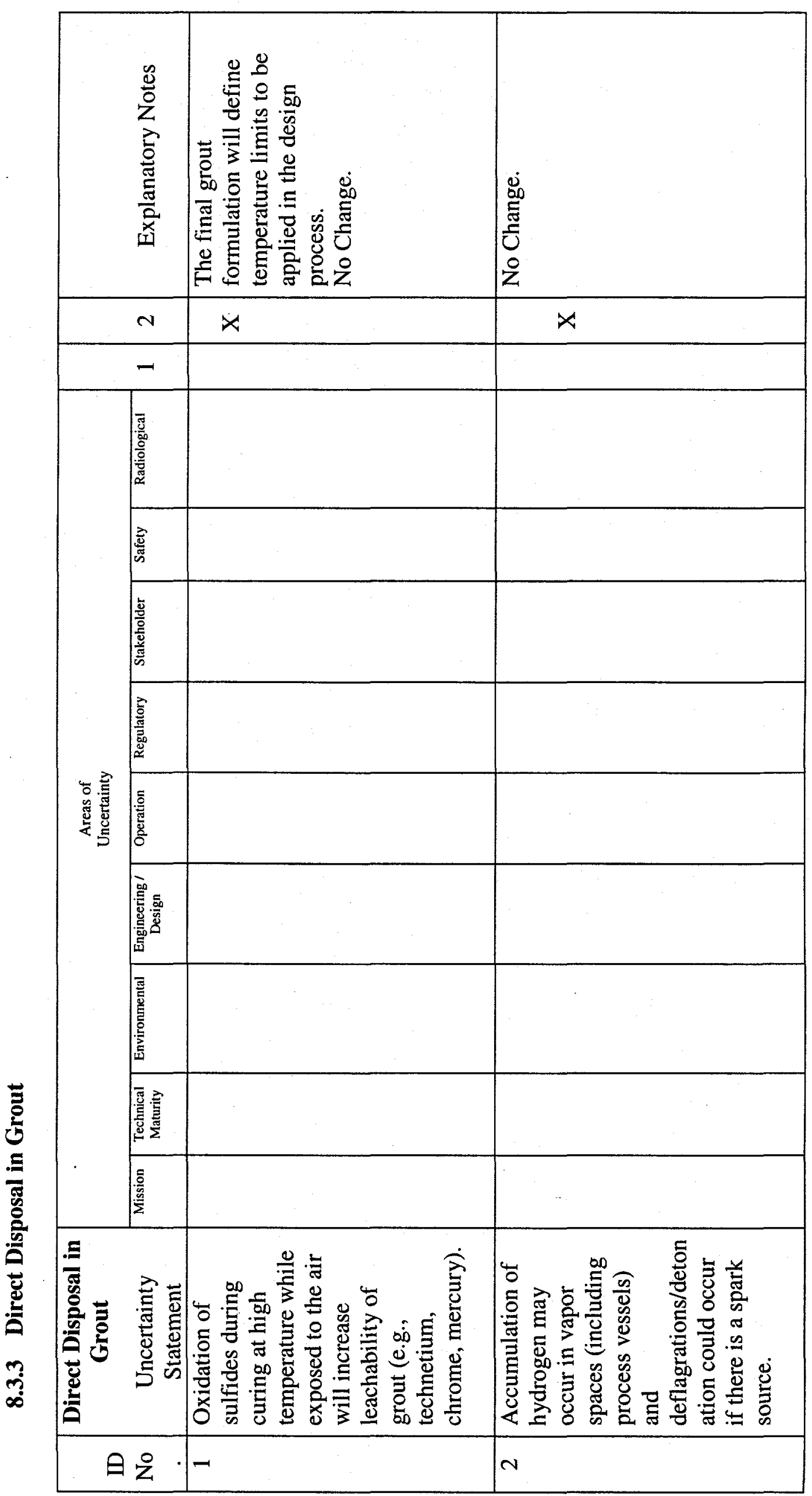


Page 237 of 277

\begin{tabular}{|c|c|c|c|c|c|c|c|c|c|c|c|c|c|}
\hline \multirow{2}{*}{$\begin{array}{l}\text { ID } \\
\text { No }\end{array}$} & \multirow{2}{*}{$\begin{array}{c}\text { Direct Disposal in } \\
\text { Grout } \\
\text { Uncertainty } \\
\text { Statement } \\
\end{array}$} & \multicolumn{9}{|c|}{$\begin{array}{l}\text { Areas of } \\
\text { Uncertainty }\end{array}$} & \multirow[b]{2}{*}{1} & \multirow[b]{2}{*}{2} & \multirow[b]{2}{*}{ Explanatory Notes } \\
\hline & & Mission & $\begin{array}{l}\text { Technical } \\
\text { Maturity }\end{array}$ & Environmental & $\begin{array}{l}\text { Engineering / } \\
\text { Design' }\end{array}$ & Operation & Regulatory & Stakeholder & Safety & Radiological & & & \\
\hline 3 & $\begin{array}{l}\mathrm{H}_{2} \text { Generation in } \\
\text { grout could } \\
\text { degrade the waste } \\
\text { form. }\end{array}$ & & & & & & & & & & & & $\begin{array}{l}\text { File } \\
\text { No Change. }\end{array}$ \\
\hline 4 & $\begin{array}{l}\text { Water expulsion } \\
\text { from monolith due } \\
\text { to displacement by } \\
\text { radiolytic gas } \\
\text { could occur. }\end{array}$ & & & & . & & & & & & & & $\begin{array}{l}\text { File } \\
\text { No Change. }\end{array}$ \\
\hline
\end{tabular}




\begin{tabular}{|c|c|c|c|c|c|c|c|c|c|c|c|c|c|}
\hline \multirow{2}{*}{$\begin{array}{l}\text { ID } \\
\text { No }\end{array}$} & \multirow{2}{*}{$\begin{array}{c}\text { Direct Disposal in } \\
\text { Grout } \\
\text { Uncertainty } \\
\text { Statement }\end{array}$} & \multicolumn{9}{|c|}{$\begin{array}{l}\text { Areas of } \\
\text { Uncertainty }\end{array}$} & \multirow[b]{2}{*}{1} & \multirow[b]{2}{*}{2} & \multirow[b]{2}{*}{ Explanatory Notes } \\
\hline & & Mission & $\begin{array}{l}\text { Technical } \\
\text { Maturity }\end{array}$ & Environmental & $\begin{array}{c}\text { Engineering ' } \\
\text { Design }\end{array}$ & Operation & Regulatory & Stakeholder & $\begin{array}{l}\text { Safety } \\
\end{array}$ & Radiological & & & \\
\hline 5 & $\begin{array}{l}\text { Existing vault } \\
\text { design may have } \\
\text { to be upgraded } \\
\text { with liners, } \\
\text { ventilation } \\
\text { upgrades, } \\
\text { temperature } \\
\text { monitoring, } \\
\text { leachate collection, } \\
\text { capping/backfillin } \\
\text { g, elimination of } \\
\text { floor penetrations, } \\
\text { HEPA filtration of } \\
\text { moist atmosphere } \\
\text { and the addition of } \\
\text { cell access for } \\
\text { failed equipment } \\
\text { disposal. }\end{array}$ & ; & & . & $\mathbf{X}$ & & & & & & $\mathbf{X}$ & & $\begin{array}{l}\text { Some of these additional } \\
\text { design features could be } \\
\text { complex and expensive. } \\
\text { No Change. }\end{array}$ \\
\hline
\end{tabular}




\begin{tabular}{|c|c|c|c|c|c|c|c|c|c|c|c|c|c|}
\hline \multirow{2}{*}{$\begin{array}{l}\text { ID } \\
\text { No }\end{array}$} & \multirow{2}{*}{$\begin{array}{c}\text { Direct Disposal in } \\
\text { Grout } \\
\text { Uncertainty } \\
\text { Statement }\end{array}$} & \multicolumn{9}{|c|}{$\begin{array}{l}\text { Areas of } \\
\text { Uncertainty }\end{array}$} & \multirow[b]{2}{*}{1} & \multirow[b]{2}{*}{2} & \multirow[b]{2}{*}{ Explanatory Notes } \\
\hline & & Mission & $\begin{array}{l}\text { Technical } \\
\text { Maturity }\end{array}$ & Environmental & 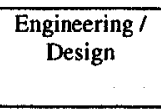 & Operation & Regulatory & Stakeholder & $\begin{array}{l}\text { Safety } \\
\end{array}$ & Radiological & & & \\
\hline 6 & $\begin{array}{l}\text { Developing a } \\
\text { formulation } \\
\text { capable of } \\
\text { maintaining } \\
\text { structural and } \\
\text { chemical integrity } \\
\text { after extended } \\
\text { curing of grout at } \\
\text { up to } 90^{\circ} \mathrm{C} \text { may } \\
\text { not be possible. }\end{array}$ & & $\mathrm{x}$ & & & & & & & & $\mathbf{X}$ & & $\begin{array}{l}\text { While the research } \\
\text { program should resolve } \\
\text { this issue, the grout } \\
\text { formulation is currently } \\
\text { unknown. Additional } \\
\text { development funding may } \\
\text { be required. } \\
\text { No Change. }\end{array}$ \\
\hline 7 & $\begin{array}{l}\text { It may be } \\
\text { necessary to } \\
\text { remove the nitrates } \\
\text { to improve the PA. }\end{array}$ & & & & & & & & & & & & $\begin{array}{l}\text { File } \\
\text { No Change. }\end{array}$ \\
\hline 8 & $\begin{array}{l}\text { If the monolith } \\
\text { cracks more due to } \\
\text { higher Cs loading, } \\
\text { does the } \\
\text { movement of } \\
\text { materials to the } \\
\text { ground water } \\
\text { increase? }\end{array}$ & & & & & & & & & & & & $\begin{array}{l}\text { File } \\
\text { No Change. } \\
\text { WSRC-TR-99-00227 }\end{array}$ \\
\hline
\end{tabular}




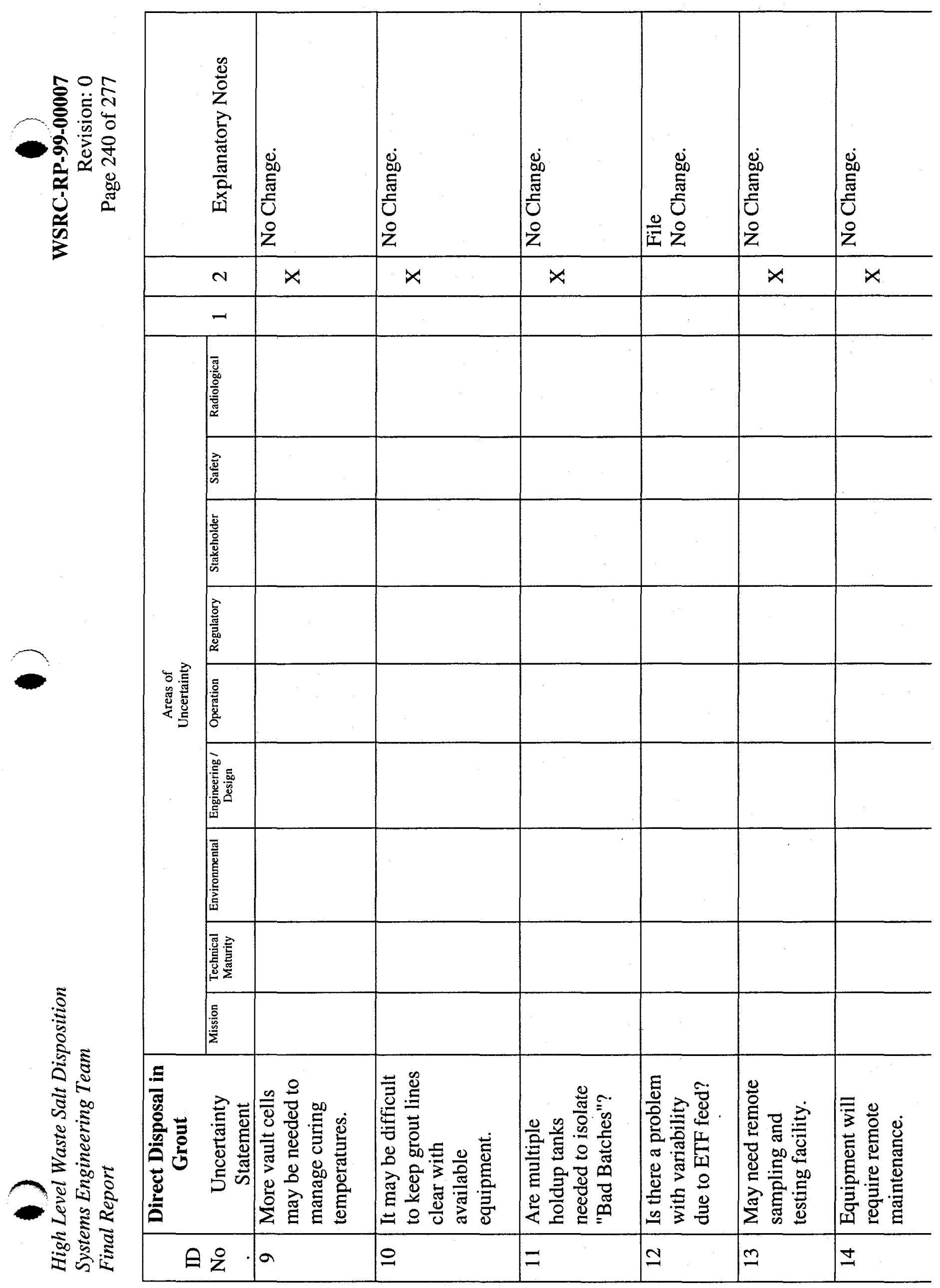




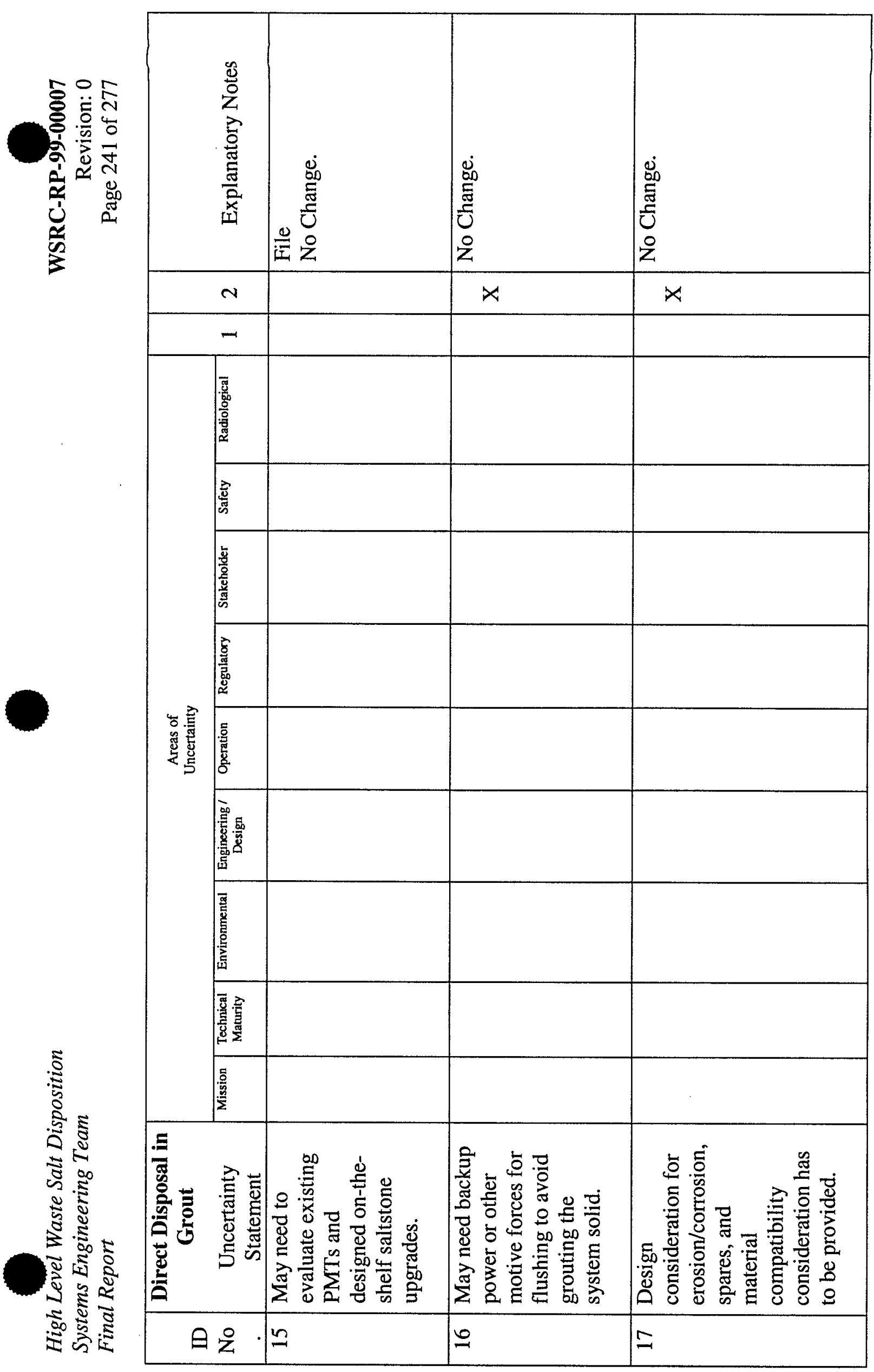


Page 242 of 277

\begin{tabular}{|c|c|c|c|c|c|c|c|c|c|c|c|c|c|}
\hline \multirow{2}{*}{$\begin{array}{l}\text { ID } \\
\text { No }\end{array}$} & \multirow{2}{*}{$\begin{array}{c}\text { Direct Disposal in } \\
\text { Grout } \\
\text { Uncertainty } \\
\text { Statement } \\
\end{array}$} & \multicolumn{9}{|c|}{$\begin{array}{l}\text { Areas of } \\
\text { Uncertainty }\end{array}$} & \multirow[b]{2}{*}{1} & \multirow[b]{2}{*}{2} & \multirow[b]{2}{*}{ Explanatory Notes } \\
\hline & & Mission & $\begin{array}{l}\text { Technical } \\
\text { Maturity }\end{array}$ & Environmental & $\begin{array}{l}\text { Engineering / } \\
\text { Design }\end{array}$ & Operation & Regulatory & Stakeholder & Safety & Radiological & & & \\
\hline 18 & $\begin{array}{l}\text { The neptunium } \\
\text { content in certain } \\
\text { HLW Tanks may } \\
\text { impact the PA and } \\
\text { WAC. }\end{array}$ & & & & & & & & & & & & $\begin{array}{l}\text { File } \\
\text { No Change. }\end{array}$ \\
\hline 19 & $\begin{array}{l}\text { Lines are cleaned } \\
\text { of blockage by } \\
\text { "Shutdown } \\
\text { Process" with } \\
\text { batch lost. Does } \\
\text { this unacceptably } \\
\text { hurt production? }\end{array}$ & & & & & & & & & & & & $\begin{array}{l}\text { File } \\
\text { No Change. }\end{array}$ \\
\hline 20 & $\begin{array}{l}\text { Operations cannot } \\
\text { "Operate" for } 8 \\
\text { hours in a } 10 \text { hour } \\
\text { day. Probably } \\
\text { need } 16 \text { hours ( } 2 \\
\text { shifts). }\end{array}$ & $\vdots$ & & & & & & & & & & & $\begin{array}{l}\text { File } \\
\text { No Change. }\end{array}$ \\
\hline 21 & $\begin{array}{l}\text { Dry material } \\
\text { delivery and } \\
\text { handling may be a } \\
\text { problem at high } \\
\text { production rates. }\end{array}$ & & . & & & & & & & & & $\mathrm{X}$ & No Change. \\
\hline
\end{tabular}


Page 243 of 277

\begin{tabular}{|c|c|c|c|c|c|c|c|c|c|c|c|c|c|}
\hline \multirow{2}{*}{$\begin{array}{l}\text { ID } \\
\text { No }\end{array}$} & \multirow{2}{*}{$\begin{array}{c}\text { Direct Disposal in } \\
\text { Grout } \\
\text { Uncertainty } \\
\text { Statement } \\
\end{array}$} & \multicolumn{9}{|c|}{$\begin{array}{l}\text { Areas of } \\
\text { Uncertainty }\end{array}$} & \multirow[b]{2}{*}{1} & \multirow[b]{2}{*}{2} & \multirow[b]{2}{*}{ Explanatory Notes } \\
\hline & & Mission & $\begin{array}{l}\text { Technical } \\
\text { Maturity }\end{array}$ & Environmental & $\begin{array}{c}\text { Engineering/ } \\
\text { Design }\end{array}$ & Operation & Regulatory & Stakeholder & Safety & Radiological & & & \\
\hline 22 & $\begin{array}{l}\text { May need to do } \\
\text { adequate cold } \\
\text { (simulant) testing } \\
\text { to support "HOT" } \\
\text { testing. }\end{array}$ & & & & & & & & & & & & $\begin{array}{l}\text { File } \\
\text { No Change. }\end{array}$ \\
\hline 23 & $\begin{array}{l}\text { Process chemistry } \\
\text { understanding and } \\
\text { application are still } \\
\text { under } \\
\text { development, } \\
\text { resulting in } 96-1 \\
\text { lessons learned not } \\
\text { yet implemented }\end{array}$ & & $\mathrm{x}$ & & & & & & & & $\mathrm{X}$ & & $\begin{array}{l}\text { Additional R\&D work } \\
\text { needed to close out } \\
\text { DNFSB } 96-1 \text { concerns. } \\
\text { No Change. }\end{array}$ \\
\hline 24 & $\begin{array}{l}\text { Remote equipment } \\
\text { handling } \\
\text { demonstration. }\end{array}$ & & & & & & & & & & & $X$ & No Change. \\
\hline 25 & $\begin{array}{l}\text { Establishment of } \\
\text { dry material } \\
\text { specifications and } \\
\text { acceptance testing } \\
\text { may be required. } \\
\text { Temperature may } \\
\text { be significant. }\end{array}$ & & & & & & & & & & & $X$ & No Change. \\
\hline
\end{tabular}


Page 244 of 277

\begin{tabular}{|c|c|c|c|c|c|c|c|c|c|c|c|c|c|}
\hline \multirow{2}{*}{$\begin{array}{l}\text { ID } \\
\text { No }\end{array}$} & \multirow{2}{*}{$\begin{array}{c}\text { Direct Disposal in } \\
\text { Grout } \\
\text { Uncertainty } \\
\text { Statement } \\
\end{array}$} & \multicolumn{9}{|c|}{$\begin{array}{l}\text { Areas of } \\
\text { Uncertainty }\end{array}$} & \multirow[b]{2}{*}{1} & \multirow[b]{2}{*}{2} & \multirow[b]{2}{*}{ Explanatory Notes } \\
\hline & & Mission & $\begin{array}{l}\text { Technical } \\
\text { Maturity }\end{array}$ & Environmental & $\begin{array}{c}\text { Engineering / } \\
\text { Design }\end{array}$ & Operation & Regulatory & Stakeholder & Safety & Radiological & & & \\
\hline 26 & $\begin{array}{l}\text { More operators } \\
\text { may be needed due } \\
\text { to saltstone layout } \\
\text { and I \& C. } \\
\text { Optimization is } \\
\text { needed. (Controls } \\
\text { location too far } \\
\text { apart. Packages } \\
\text { don't } \\
\text { communicate). }\end{array}$ & & & & & & & & & & & $\mathrm{X}$ & No Change. \\
\hline 27 & $\begin{array}{l}\text { Are there radiation } \\
\text { effects on } \\
\text { equipment in } \\
\text { Vault? CCTVs, } \\
\text { wiring, gaskets, } \\
\text { seals, insulation, } \\
\text { etc. (Disposable } \\
\text { TV's vs RAD- } \\
\text { Hardened). }\end{array}$ & & & & & & & & & & & $\mathrm{X}$ & No Change. \\
\hline 28 & $\begin{array}{l}\text { "Hot" testing to } \\
\text { verify process } \\
\text { chemistry may be } \\
\text { needed. }\end{array}$ & & & & & & & & & & & & $\begin{array}{l}\text { File } \\
\text { No Change. }\end{array}$ \\
\hline
\end{tabular}




\begin{tabular}{|c|c|c|c|c|c|c|c|c|c|c|c|c|c|}
\hline \multirow{2}{*}{$\begin{array}{l}\text { ID } \\
\text { No }\end{array}$} & \multirow{2}{*}{$\begin{array}{c}\text { Direct Disposal in } \\
\text { Grout } \\
\text { Uncertainty } \\
\text { Statement } \\
\end{array}$} & \multicolumn{9}{|c|}{$\begin{array}{l}\text { Arcas of } \\
\text { Uncertainty }\end{array}$} & \multirow[b]{2}{*}{1} & \multirow[b]{2}{*}{2} & \multirow[b]{2}{*}{ Explanatory Notes } \\
\hline & & Mission & $\begin{array}{l}\text { Technical } \\
\text { Maturity }\end{array}$ & Environmental & $\begin{array}{l}\text { Engineering / } \\
\text { Design }\end{array}$ & Operation & Regulatory & Stakeholder & Safety & Radiological & & & \\
\hline 29 & $\begin{array}{l}\text { Cs-135 } \\
\text { concentrations } \\
\text { may impact the } \\
\text { Performance } \\
\text { Assessment (PA). }\end{array}$ & & & & & & $\mathbf{X}$ & & & & $\mathbf{x}$ & & $\begin{array}{l}\text { Current PA does not } \\
\text { address Cs-135. } \\
\text { No Change. }\end{array}$ \\
\hline 30 & $\begin{array}{l}\text { If process design } \\
\text { matures prior to } \\
\text { grout formulation } \\
\text { the product quality } \\
\text { may be at risk. }\end{array}$ & & & & & & & . & & & & & $\begin{array}{l}\text { File } \\
\text { No Change. }\end{array}$ \\
\hline 31 & $\begin{array}{l}\text { Difficulty in } \\
\text { filtration of sludge } \\
\text { and/or MST will } \\
\text { produce low } \\
\text { filtrate flow rates } \\
\text { and require } \\
\text { frequent cleaning }\end{array}$ & 1 & $\mathrm{x}$ & & & & & & & & $\mathrm{X}$ & & $\begin{array}{l}\text { R\&D work has confirmed } \\
\text { this risk. } \\
\text { WSRC-TR-99-00346 }\end{array}$ \\
\hline 32 & $\begin{array}{l}\text { TRU } \\
\text { decontamination } \\
\text { with MST is not } \\
\text { adequate. }\end{array}$ & & $\mathbf{X}$ & & & & & & & & $X$ & & $\begin{array}{l}\text { Kinetics for bounding } \\
\text { plutonium is too slow. } \\
\text { WSRC-TR-99-00219 } \\
\text { WSRC-TR-99-00286 }\end{array}$ \\
\hline
\end{tabular}


Page 246 of 277

\begin{tabular}{|c|c|c|c|c|c|c|c|c|c|c|c|c|c|}
\hline \multirow{2}{*}{$\begin{array}{l}\text { ID } \\
\text { No }\end{array}$} & \multirow{2}{*}{$\begin{array}{c}\text { Direct Disposal in } \\
\text { Grout } \\
\text { Uncertainty } \\
\text { Statement } \\
\end{array}$} & \multicolumn{9}{|c|}{$\begin{array}{l}\text { Areas of } \\
\text { Uncertainty }\end{array}$} & \multirow[b]{2}{*}{1} & \multirow[b]{2}{*}{2} & \multirow[b]{2}{*}{ Explanatory Notes } \\
\hline & & Mission & $\begin{array}{l}\text { Technical } \\
\text { Maturity }\end{array}$ & Environmental & $\begin{array}{c}\text { Engineering/ } \\
\text { Design }\end{array}$ & Operation & Regulatory & Stakeholder & Safety & Radiological & & & \\
\hline 33 & $\begin{array}{l}\text { MST amount } \\
\text { needed for } \\
\text { decontamination } \\
\text { exceeds DWPF Ti } \\
\text { glass limits. }\end{array}$ & & & & & & & & & & & $\mathrm{X}$ & $\begin{array}{l}\text { MST concentration of } 0.4 \\
\text { g/L makes acceptable } \\
\text { glass. } \\
\text { WSRC-TR-99-00332 }\end{array}$ \\
\hline 34 & $\begin{array}{l}\text { TRU } \\
\text { decontamination } \\
\text { may require } \\
\text { excessive MST. }\end{array}$ & & $\mathrm{X}$ & & & & & & & & $X$ & & $\begin{array}{l}\text { Equilibrium capacity } \\
\text { shown to be sufficient by } \\
\text { R\&D. Neptunium for } \\
\text { Tanks } 33 \text { and } 34 \text { will } \\
\text { require blending. } \\
\text { WSRC-TR-99-00219 } \\
\text { WSRC-TR-99-00286 }\end{array}$ \\
\hline 35 & $\begin{array}{l}\text { Inability to } \\
\text { develop a grout } \\
\text { formulation for } \\
\text { increased Cs \& K } \\
\text { and Salt Molarity } \\
\text { concentration. }\end{array}$ & i & & & & & & & & & & $\mathrm{X}$ & No change. \\
\hline 36 & $\begin{array}{l}\text { Grout temperature } \\
\text { too high to make } \\
\text { acceptable grout }\end{array}$ & & & & & & & & & & & $\mathrm{X}$ & $\begin{array}{l}\text { No change. } \\
\text { WSRC-TR-99-00227 }\end{array}$ \\
\hline
\end{tabular}


Page 247 of 277

\begin{tabular}{|c|c|c|c|c|c|c|c|c|c|c|c|c|c|}
\hline \multirow{2}{*}{$\begin{array}{l}\text { ID } \\
\text { No }\end{array}$} & \multirow{2}{*}{$\begin{array}{c}\text { Direct Disposal in } \\
\text { Grout } \\
\text { Uncertainty } \\
\text { Statement } \\
\end{array}$} & \multicolumn{9}{|c|}{$\begin{array}{l}\text { Areas of } \\
\text { Uncertainty }\end{array}$} & \multirow[b]{2}{*}{1} & \multirow[b]{2}{*}{2} & \multirow[b]{2}{*}{ Explanatory Notes } \\
\hline & & $\overline{\text { Mission }}$ & $\begin{array}{l}\text { Technical } \\
\text { Maturity }\end{array}$ & Environmental & $\begin{array}{l}\text { Engineering / } \\
\text { Design }\end{array}$ & Operation & Regulatory & Stakeholder & $\begin{array}{l}\text { Safety } \\
\text {. }\end{array}$ & Radiological & & & \\
\hline 37 & $\begin{array}{l}\text { Existing grout } \\
\text { equipment requires } \\
\text { hands on } \\
\text { maintenance } \\
\text { negatively } \\
\text { affecting potential } \\
\text { personnel } \\
\text { exposure and } \\
\text { attainment. }\end{array}$ & & & & & & & & & & & $\mathrm{X}$ & No change. \\
\hline 38 & $\begin{array}{l}\text { Difficulty in } \\
\text { resuspending MST } \\
\text { after long } \\
\text { quiescent period }\end{array}$ & & & & & & & & & & & $X$ & $\begin{array}{l}\text { After } 60 \text { days of settling, } \\
\text { resuspension was not } \\
\text { achieved with proposed } \\
\text { flowsheet design. } \\
\text { ORNL-TM-1999/166 }\end{array}$ \\
\hline 39 & $\begin{array}{l}\text { HLW cannot } \\
\text { support tank } \\
\text { blending strategies } \\
\text { for Cs (or other } \\
\text { species) to } \\
\text { support process } \\
\text { requirements. }\end{array}$ & i & & & & & & & & & & $\mathrm{X}$ & No change. \\
\hline 40 & $\begin{array}{l}\text { GT73 resin will be } \\
\text { selective to Co60 } \\
\text { or other trace } \\
\text { radioisotopes. }\end{array}$ & & & & & & & & & & & & File \\
\hline
\end{tabular}


Page 248 of 277

\begin{tabular}{|c|c|c|c|c|c|c|c|c|c|c|c|c|c|}
\hline \multirow{2}{*}{$\begin{array}{l}\text { ID } \\
\text { No }\end{array}$} & \multirow{2}{*}{$\begin{array}{c}\text { Direct Disposal in } \\
\text { Grout } \\
\text { Uncertainty } \\
\text { Statement }\end{array}$} & \multicolumn{9}{|c|}{$\begin{array}{l}\text { Areas of } \\
\text { Uncertainty }\end{array}$} & \multirow[b]{2}{*}{1} & \multirow[b]{2}{*}{2} & \multirow[b]{2}{*}{ Explanatory Notes } \\
\hline & & Mission & $\begin{array}{l}\text { Technical } \\
\text { Maturity }\end{array}$ & Environmental & $\begin{array}{c}\text { Engineering/ } \\
\text { Design }\end{array}$ & Operation & Regulatory & Stakeholder & Safety & Radiological & & & \\
\hline 41 & $\begin{array}{l}\text { No ability to } \\
\text { dispose of failed } \\
\text { contaminated } \\
\text { equipment. }\end{array}$ & & & & & & & & & & & & $\begin{array}{l}\text { File } \\
\text { No change. }\end{array}$ \\
\hline 42 & $\begin{array}{l}\text { Burn, deflagration, } \\
\text { detonation to } \\
\text { radiolytic } \mathrm{H} 2 \text { in } \\
\text { the vaults. }\end{array}$ & & & & & & & & & & & $\mathrm{X}$ & No change. \\
\hline 43 & $\begin{array}{l}\text { Loss of grout } \\
\text { cooling results in } \\
\text { organics and/or Cs } \\
\text { releases }\end{array}$ & & & & & & & & & & & $X$ & $\begin{array}{l}\text { No change. } \\
\text { WSRC-TR-99-00227 }\end{array}$ \\
\hline 44 & $\begin{array}{l}\text { Seismic event } \\
\text { results in a slurry } \\
\text { spill. }\end{array}$ & & & & & & & & & & & $\mathrm{X}$ & No change. \\
\hline 45 & $\begin{array}{l}\text { The volume of } \\
\text { Class C waste } \\
\text { exceeds the NRC } \\
\text { interpretation for } \\
\text { percent of low } \\
\text { level waste and } \\
\text { will not be } \\
\text { allowed. }\end{array}$ & & & & $\because$ & & & & & & & & $\begin{array}{l}\text { File } \\
\text { No change. }\end{array}$ \\
\hline
\end{tabular}




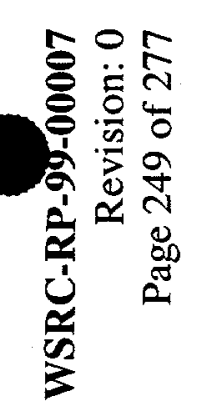

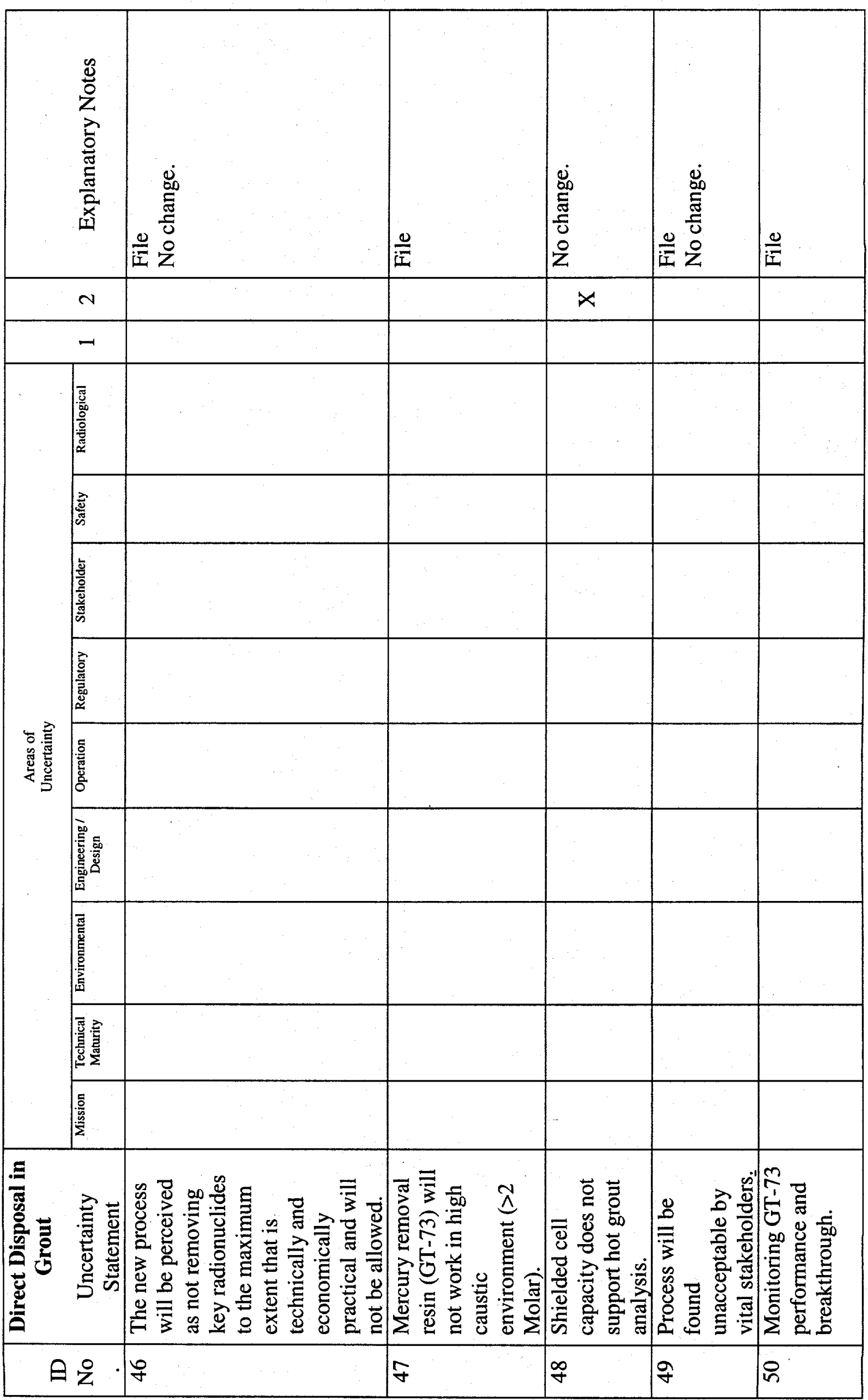


Page 250 of 277

\begin{tabular}{|c|c|c|c|c|c|c|c|c|c|c|c|c|c|}
\hline \multirow{2}{*}{$\begin{array}{l}\text { ID } \\
\text { No }\end{array}$} & \multirow{2}{*}{$\begin{array}{c}\text { Direct Disposal in } \\
\text { Grout } \\
\text { Uncertainty } \\
\text { Statement }\end{array}$} & \multicolumn{9}{|c|}{$\begin{array}{l}\text { Areas of } \\
\text { Uncertainty }\end{array}$} & \multirow[b]{2}{*}{1} & \multirow[b]{2}{*}{2} & \multirow[b]{2}{*}{ Explanatory Notes } \\
\hline & & Mission & $\begin{array}{l}\text { Technical } \\
\text { Maturity }\end{array}$ & Environmental & $\begin{array}{c}\text { Engineering/ } \\
\text { Design }\end{array}$ & Operation & Regulatory & Stakeholder & Safety & Radiological & & & \\
\hline 51 & $\begin{array}{l}\text { Temperature } \\
\text { changes or } \\
\text { chemistry changes } \\
\text { may cause post } \\
\text { precipitation after } \\
\text { the MST strike. }\end{array}$ & & & & & & & & & & & & $\begin{array}{l}\text { File } \\
\text { No change. }\end{array}$ \\
\hline 52 & $\begin{array}{l}\text { Hydrogen control } \\
\text { in the MST strike } \\
\text { process. }\end{array}$ & & & & & & & & & & & $\mathrm{X}$ & No change. \\
\hline 53 & $\begin{array}{l}\text { Analysis delay (1 } \\
\text { week) in } \\
\text { measuring for } S_{\mathrm{r}} \\
\text { DF in MST } \\
\text { process. }\end{array}$ & & $\mathrm{x}$ & & & & & & & & $X$ & & $\begin{array}{l}\text { Requires new analytical } \\
\text { techniques. } \\
\text { No change. }\end{array}$ \\
\hline 54 & $\begin{array}{l}\text { Fate and } \\
\text { downstream } \\
\text { impact of oxalate, } \\
\text { after a cross flow } \\
\text { filter cleaning } \\
\text { operation }\end{array}$ & & & & & & & & & & & & $\begin{array}{l}\text { File } \\
\text { No change. }\end{array}$ \\
\hline
\end{tabular}


Page 251 of 277

\begin{tabular}{|c|c|c|c|c|c|c|c|c|c|c|c|c|c|}
\hline ID & $\begin{array}{c}\text { Direct Disposal in } \\
\text { Grout }\end{array}$ & & & & & $\begin{array}{l}\text { Areas o } \\
\text { Uncertain }\end{array}$ & & & & . & & & \\
\hline No & $\begin{array}{c}\text { Uncertainty } \\
\text { Statement }\end{array}$ & Mission & $\begin{array}{l}\text { Technical } \\
\text { Maturity }\end{array}$ & Environmental & $\begin{array}{c}\text { Engineering / } \\
\text { Design }\end{array}$ & Operation & Regulatory & Stakeholder & $\begin{array}{l}\text { Safety } \\
\text { Sal }\end{array}$ & Radiological & 1 & 2 & Explanatory Notes \\
\hline 55 & $\begin{array}{l}\text { Rate of spent } \\
\text { equipment } \\
\text { generation and its } \\
\text { disposal. }\end{array}$ & & & & & & & & & & & $\mathrm{X}$ & No change. \\
\hline 56 & $\begin{array}{l}\text { Minimize spark } \\
\text { sources in } \mathrm{H}_{2} \text { rich } \\
\text { areas. }\end{array}$ & & & & & & & & & & & $X$ & No change. \\
\hline 57 & $\begin{array}{l}\text { What is the fate of } \\
\text { Am in the process? }\end{array}$ & & $\mathrm{x}$ & & & & & & & & $\mathbf{X}$ & & $\begin{array}{l}\text { Does MST strike affect } \\
\text { Am disposition? } \\
\text { No change. }\end{array}$ \\
\hline 58 & $\begin{array}{l}\text { Process sampling } \\
\text { strategy for } \\
\text { Material Control } \\
\text { and Quality. }\end{array}$ & & & & & & & & & & & $X$ & No change. \\
\hline 59 & $\begin{array}{l}\text { Cold Chemical } \\
\text { Storage controls } \\
\text { for shelf life } \\
\text { concerns. }\end{array}$ & & & & & & & & & & & & $\begin{array}{l}\text { File } \\
\text { No change. }\end{array}$ \\
\hline 60 & $\begin{array}{l}\text { Tank capacity } \\
\text { requirements to } \\
\text { support } \\
\text { "Drainback" } \\
\text { concerns. }\end{array}$ & & & & & & & & & & & $X$ & No change. \\
\hline
\end{tabular}




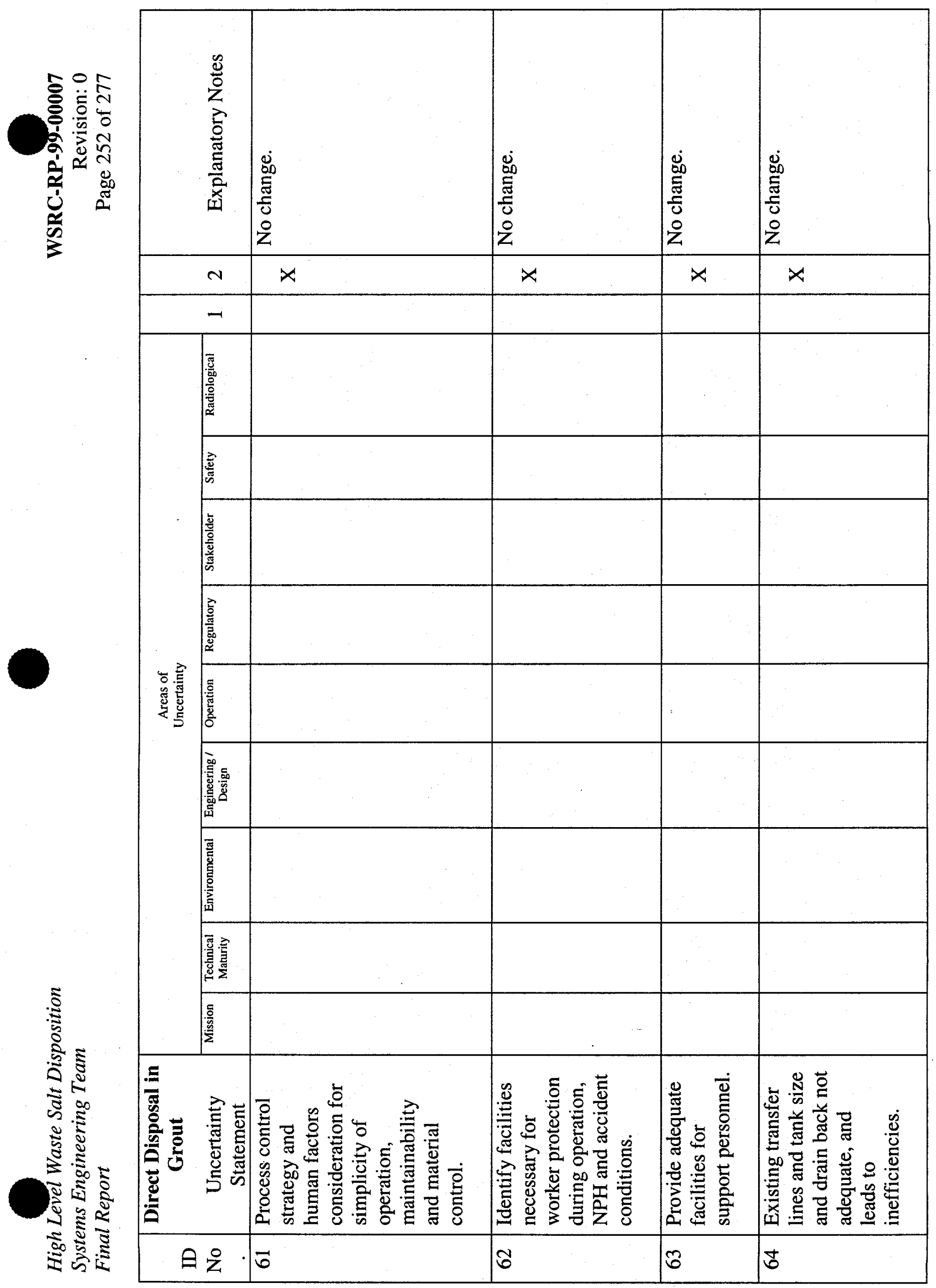




\begin{tabular}{|c|c|c|c|c|c|c|c|c|c|c|c|c|c|}
\hline \multirow{2}{*}{$\begin{array}{l}\text { ID } \\
\text { No }\end{array}$} & \multirow{2}{*}{$\begin{array}{c}\text { Direct Disposal in } \\
\text { Grout } \\
\text { Uncertainty } \\
\text { Statement } \\
\end{array}$} & \multicolumn{9}{|c|}{$\begin{array}{l}\text { Areas of } \\
\text { Uncertainty }\end{array}$} & \multirow[b]{2}{*}{1} & \multirow[b]{2}{*}{2} & \multirow[b]{2}{*}{ Explanatory Notes } \\
\hline & & Mission & $\begin{array}{l}\text { Technical } \\
\text { Maturity }\end{array}$ & Environmental & $\begin{array}{l}\text { Engineering / } \\
\text { Design }\end{array}$ & Operation & Regulatory & Stakeholder & Safety & Radiological & & & \\
\hline 65 & $\begin{array}{l}\text { Solids on filters } \\
\text { will not dissolve or } \\
\text { would be difficult } \\
\text { to dissolve in } \\
\text { oxalic acid. }\end{array}$ & & & & & & & & & & & & $\begin{array}{l}\text { File } \\
\text { No change. }\end{array}$ \\
\hline 66 & $\begin{array}{l}\text { Require adequate } \\
\text { size of remote and } \\
\text { contact } \\
\text { decontamination } \\
\text { cells, with } \\
\text { adequate crane } \\
\text { coverage. }\end{array}$ & & & . & & & & & & & & $\mathrm{X}$ & No change. \\
\hline 67 & $\begin{array}{l}\text { Need for docking } \\
\text { door to maintain } \\
\text { clean crane } \\
\text { controls and } \\
\text { electronics. }\end{array}$ & ; & & & & & & & & & & $\mathrm{X}$ & No change. \\
\hline 68 & $\begin{array}{l}\text { Adequate process } \\
\text { instrumentation to } \\
\text { detect process } \\
\text { upsets and perform } \\
\text { routine } \\
\text { monitoring. }\end{array}$ & & & & & & & & & & & $X$ & No change. \\
\hline
\end{tabular}




\subsubsection{Small Tank TPB Precipitation}

\begin{tabular}{|c|c|c|c|c|c|c|c|c|c|c|c|c|c|}
\hline \multirow{2}{*}{$\begin{array}{l}\text { ID } \\
\text { No }\end{array}$} & \multirow{2}{*}{$\begin{array}{c}\text { Small Tank TPB } \\
\text { Precipitation } \\
\text { Uncertainty } \\
\text { Statement } \\
\end{array}$} & \multicolumn{9}{|c|}{$\begin{array}{l}\text { Areas of } \\
\text { Uncertainty }\end{array}$} & \multirow[b]{2}{*}{1} & \multirow[b]{2}{*}{2} & \multirow[b]{2}{*}{$\begin{array}{c}\text { Explanatory } \\
\text { Notes }\end{array}$} \\
\hline & & Mission & $\begin{array}{l}\text { Technical } \\
\text { Maturity }\end{array}$ & Environmental & $\begin{array}{c}\text { Enginecring / } \\
\text { Design }\end{array}$ & Operation & Regulatory & Stakeholder & Safety & $\begin{array}{l}\text { Radiological } \\
\end{array}$ & & & \\
\hline 1 & $\begin{array}{l}\text { Loss of cooling } \\
\text { event - How hot } \\
\text { will the precipitate } \\
\text { get from the } \\
\text { Cesium decay heat } \\
\text { and resulting } \\
\text { benzene } \\
\text { generation? }\end{array}$ & & & & 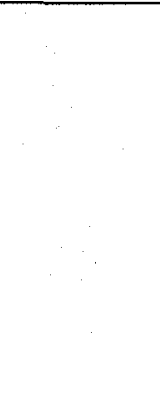 & & & & & & & $\mathrm{X}$ & No change. \\
\hline 2 & $\begin{array}{l}\text { Benzene release } \\
\text { after power loss } \\
\text { could result in } \\
\text { exceeding LFL in } \\
\text { the process cells. }\end{array}$ & i & & & & & & & & . & & $\mathrm{X}$ & No change. \\
\hline 3 & $\begin{array}{l}\text { Does MST carry } \\
\text { down catalysts or } \\
\text { concentrate them? }\end{array}$ & & & & & & & & & & & & $\begin{array}{l}\text { File } \\
\text { No change. }\end{array}$ \\
\hline
\end{tabular}




\begin{tabular}{|c|c|c|c|c|c|c|c|c|c|c|c|c|c|}
\hline \multirow{2}{*}{$\begin{array}{l}\text { ID } \\
\text { No }\end{array}$} & \multirow{2}{*}{$\begin{array}{c}\text { Small Tank TPB } \\
\text { Precipitation } \\
\text { Uncertainty } \\
\text { Statement } \\
\end{array}$} & \multicolumn{9}{|c|}{$\begin{array}{c}\text { Areas of } \\
\text { Uncertainty }\end{array}$} & \multirow[b]{2}{*}{1} & \multirow[b]{2}{*}{2} & \multirow[b]{2}{*}{$\begin{array}{l}\text { Explanatory } \\
\text { Notes }\end{array}$} \\
\hline & & Mission & $\begin{array}{l}\text { Technical } \\
\text { Maturity }\end{array}$ & Environmental & $\begin{array}{c}\text { Engineering / } \\
\text { Design }\end{array}$ & Operation & Regulatory & Stakeholder & Safety & Radiological & & & \\
\hline 4 & $\begin{array}{l}\text { Close coupled unit } \\
\text { operations adds } \\
\text { production } \\
\text { complexity. Salt } \\
\text { Cell in DWPF has } \\
\text { to be operated in } \\
\text { this option. }\end{array}$ & & & & & $\mathbf{X}$ & & & & & $\mathbf{X}$ & & $\begin{array}{l}\text { Assumes } \\
\text { undemonstrated } \\
\text { operation } \\
\text { efficiencies in the } \\
\text { LCC basis. } \\
\text { Hydrolysis steps } \\
\text { could be } \\
\text { performed in new } \\
\text { facility. } \\
\text { No change. }\end{array}$ \\
\hline 5 & $\begin{array}{l}\text { How do you } \\
\text { recover from a } \\
\text { batch that } \\
\text { decomposes? Need } \\
\text { capability to } \\
\text { deinventory tanks } \\
\text { and recycle. }\end{array}$ & & & & & $\mathrm{x}$ & & & & & $X$ & & $\begin{array}{l}\text { Catalyst } \\
\text { activation greater } \\
\text { than the bounding } \\
\text { case results in a } \\
\text { loss of product } \\
\text { DF. }\end{array}$ \\
\hline 6 & $\begin{array}{l}\text { If the Batch } \\
\text { Decomposes, } \\
\text { recycling will only } \\
\text { repeat the problem. } \\
\text { Need a hold tank to } \\
\text { treat. }\end{array}$ & & & & & $\mathrm{x}$ & & & & & $X$ & & $\begin{array}{l}\text { Catalyst } \\
\text { activation greater } \\
\text { than the bounding } \\
\text { case results in a } \\
\text { loss of product } \\
\text { DF. }\end{array}$ \\
\hline
\end{tabular}




\begin{tabular}{|c|c|c|c|c|c|c|c|c|c|c|c|c|c|}
\hline \multirow{2}{*}{$\begin{array}{l}\text { ID } \\
\text { No }\end{array}$} & \multirow{2}{*}{$\begin{array}{c}\text { Small Tank TPB } \\
\text { Precipitation } \\
\text { Uncertainty } \\
\text { Statement }\end{array}$} & \multicolumn{9}{|c|}{$\begin{array}{l}\text { Areas of } \\
\text { Uncertainty }\end{array}$} & \multirow[b]{2}{*}{1} & \multirow[b]{2}{*}{2} & \multirow[b]{2}{*}{$\begin{array}{l}\text { Explanatory } \\
\text { Notes }\end{array}$} \\
\hline & & Mission & $\begin{array}{l}\text { Technical } \\
\text { Maturity }\end{array}$ & Environmental & $\begin{array}{c}\text { Engineering / } \\
\text { Design }\end{array}$ & Operation & Regulatory & Stakeholder & Safety & Radiological & & & \\
\hline 7 & $\begin{array}{l}\text { Benzene releases } \\
\text { may exceed permit } \\
\text { levels due to } \\
\text { additional } \\
\text { (unknown) catalytic } \\
\text { effects or catalyst } \\
\text { build-up through } \\
\text { plate-out. }\end{array}$ & & $\mathbf{X}$ & & & & & & & & $\mathbf{x}$ & & $\begin{array}{l}\text { Uncertainty } \\
\text { dependent on } \\
\text { R\&D results. } \\
\text { Minor if permit } \\
\text { limits are } \\
\text { protected. } \\
\text { Catalyst } \\
\text { activation greater } \\
\text { than the bounding } \\
\text { case results in a } \\
\text { loss of product } \\
\text { DF. } \\
\text { No change. }\end{array}$ \\
\hline 8 & $\begin{array}{l}\text { How do we know } \\
\text { when we get to } \\
10 \% \text { precipitate } \\
\text { concentration? }\end{array}$ & : & & & & & & & & & & & $\begin{array}{l}\text { File } \\
\text { No change. }\end{array}$ \\
\hline 9 & $\begin{array}{l}\text { Process sampling } \\
\text { strategy for } \\
\text { Material Control } \\
\text { and Quality. }\end{array}$ & & & & & & & & & & & $X$ & No change. \\
\hline 10 & $\begin{array}{l}\text { Instrumentation } \\
\text { control for safety } \\
\text { protection strategy. }\end{array}$ & . & & & & & & & & & & $\mathrm{X}$ & No change. \\
\hline
\end{tabular}


Page 257 of 277

\begin{tabular}{|c|c|c|c|c|c|c|c|c|c|c|c|c|c|}
\hline \multirow{2}{*}{$\begin{array}{l}\text { ID } \\
\text { No }\end{array}$} & \multirow{2}{*}{$\begin{array}{c}\text { Small Tank TPB } \\
\text { Precipitation } \\
\text { Uncertainty } \\
\text { Statement } \\
\end{array}$} & \multicolumn{9}{|c|}{$\begin{array}{c}\text { Areas of } \\
\text { Uncertainty }\end{array}$} & \multirow[b]{2}{*}{1} & \multirow[b]{2}{*}{2} & \multirow[b]{2}{*}{$\begin{array}{c}\text { Explanatory } \\
\text { Notes }\end{array}$} \\
\hline & & Mission & $\begin{array}{l}\text { Technical } \\
\text { Maturity }\end{array}$ & Environmental & $\begin{array}{c}\text { Engineering' } \\
\text { Designg }\end{array}$ & Operation & Regulatory & Stakeholder & Safety & Radiological & & & \\
\hline 11 & $\begin{array}{l}\text { How long is the } \\
\text { storage time for the } \\
\text { NaTPB? Shelf life } \\
\text { and benzene } \\
\text { release. }\end{array}$ & & & & & & & & & & & & $\begin{array}{l}\text { File } \\
\text { No change. }\end{array}$ \\
\hline 12 & $\begin{array}{l}\text { Ventilation } \\
\text { considerations for } \\
\text { vessel ventilation } \\
\text { addressing material } \\
\text { carry over to } \\
\text { filtration material. } \\
\text { Potential for } \\
\text { organics and } \\
\text { nitrates in the } \\
\text { HEPA filters and } \\
\text { ventilation system. }\end{array}$ & : & & & & . & & & & & & $X$ & No change. \\
\hline 13 & $\begin{array}{l}\text { Training for } \\
\text { maintenance and } \\
\text { operations } \\
\text { personnel to } \\
\text { support facility } \\
\text { operation } \\
\text { (unique equipment } \\
\text { and instruments). }\end{array}$ & & & & & & & & & & & & $\begin{array}{l}\text { File } \\
\text { No change. }\end{array}$ \\
\hline
\end{tabular}




\begin{tabular}{|c|c|c|c|c|c|c|c|c|c|c|c|c|c|}
\hline \multirow{2}{*}{$\begin{array}{l}\text { ID } \\
\text { No }\end{array}$} & \multirow{2}{*}{$\begin{array}{c}\text { Small Tank TPB } \\
\text { Precipitation } \\
\text { Uncertainty } \\
\text { Statement } \\
\end{array}$} & \multicolumn{9}{|c|}{$\begin{array}{l}\text { Areas of } \\
\text { Uncertainty }\end{array}$} & \multirow[b]{2}{*}{1} & \multirow[b]{2}{*}{2} & \multirow[b]{2}{*}{$\begin{array}{c}\text { Explanatory } \\
\text { Notes }\end{array}$} \\
\hline & & Mission & $\begin{array}{l}\text { Technical } \\
\text { Maturity }\end{array}$ & Environmental & $\begin{array}{l}\text { Engineering/ } \\
\text { Design }\end{array}$ & Operation & Regulatory & Stakeholder & Safety & $\begin{array}{l}\text { Radiological } \\
\end{array}$ & & & \\
\hline 14 & $\begin{array}{l}\text { Cold Chemical } \\
\text { Storage controls for } \\
\text { shelf life concerns. }\end{array}$ & & & & & & & & & & & & $\begin{array}{l}\text { File } \\
\text { No change. }\end{array}$ \\
\hline 15 & $\begin{array}{l}\text { Tank In-Leakage } \\
\text { impacts of process } \\
\text { chemistry controls. }\end{array}$ & & & & & & & & & & & $\mathrm{X}$ & No change. \\
\hline 16 & $\begin{array}{l}\text { Material settles or } \\
\text { plates out in the } \\
\text { tank and } \\
\text { concentrates. }\end{array}$ & & & & & & & & & & & & $\begin{array}{l}\text { File } \\
\text { No change. }\end{array}$ \\
\hline 17 & $\begin{array}{l}\text { Difficulty to } \\
\text { transfer } 10 \mathrm{~W} \% \\
\text { slurry to DWPF } \\
\text { because of high } \\
\text { viscosity. }\end{array}$ & $:$ & & & & & & & & & & $\mathrm{X}$ & $\begin{array}{l}\text { No change. } \\
\text { WSRC-TR-99- } \\
00243\end{array}$ \\
\hline 18 & $\begin{array}{l}\text { Effect of materials } \\
\text { of construction on } \\
\text { catalytic effect. }\end{array}$ & & & & & & & & & & & $X$ & No change. \\
\hline
\end{tabular}




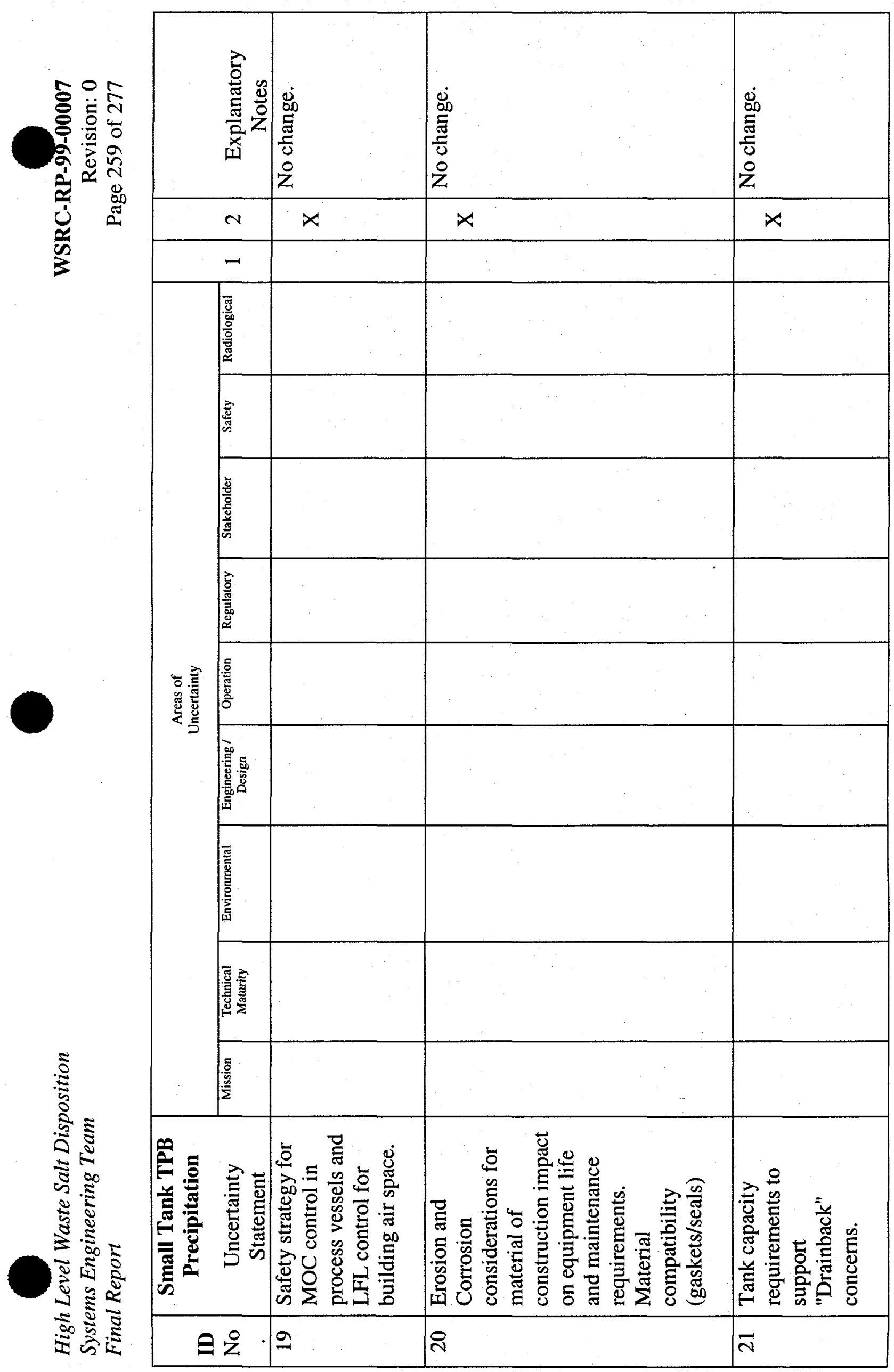


Page 260 of 277

\begin{tabular}{|c|c|c|c|c|c|c|c|c|c|c|c|c|c|}
\hline \multirow{2}{*}{$\begin{array}{l}\text { ID } \\
\text { No }\end{array}$} & \multirow{2}{*}{$\begin{array}{c}\text { Small Tank TPB } \\
\text { Precipitation } \\
\text { Uncertainty } \\
\text { Statement } \\
\end{array}$} & \multicolumn{9}{|c|}{$\begin{array}{l}\text { Areas of } \\
\text { Uncertainty }\end{array}$} & \multirow[b]{2}{*}{1} & \multirow[b]{2}{*}{2} & \multirow[b]{2}{*}{$\begin{array}{c}\text { Explanatory } \\
\text { Notes }\end{array}$} \\
\hline & & Mission & $\begin{array}{l}\text { Technical } \\
\text { Maturity }\end{array}$ & Environmental & $\begin{array}{c}\text { Engineering/ } \\
\text { Design }\end{array}$ & Operation & Regulatory & Stakeholder & Safety & Radiological & & & \\
\hline 22 & $\begin{array}{l}\text { Safety strategy for } \\
\text { transfer paths and } \\
\text { leak detection } \\
\text { (benzene } \\
\text { accumulation). }\end{array}$ & & & & & & & & & & & $\mathrm{X}$ & No change. \\
\hline 23 & $\begin{array}{l}\text { STPB impurities } \\
\text { and quality control } \\
\text { for impact on the } \\
\text { process. }\end{array}$ & & & & & & & & & & & & $\begin{array}{l}\text { File } \\
\text { No change. }\end{array}$ \\
\hline 24 & $\begin{array}{l}\text { Benzene chronic } \\
\text { release problems. } \\
\text { Do not over design } \\
\text { for worker } \\
\text { exposure based on } \\
\text { AB or Safety } \\
\text { Assumptions. }\end{array}$ & & . & & & & & . & & & & & $\begin{array}{l}\text { File } \\
\text { No change. }\end{array}$ \\
\hline 25 & $\begin{array}{l}\text { Consider } \\
\text { modifications for } \\
\text { future benzene } \\
\text { abatement in the } \\
\text { current design } \\
\text { process. }\end{array}$ & & & & & & & & & & & & $\begin{array}{l}\text { File } \\
\text { No change. }\end{array}$ \\
\hline
\end{tabular}




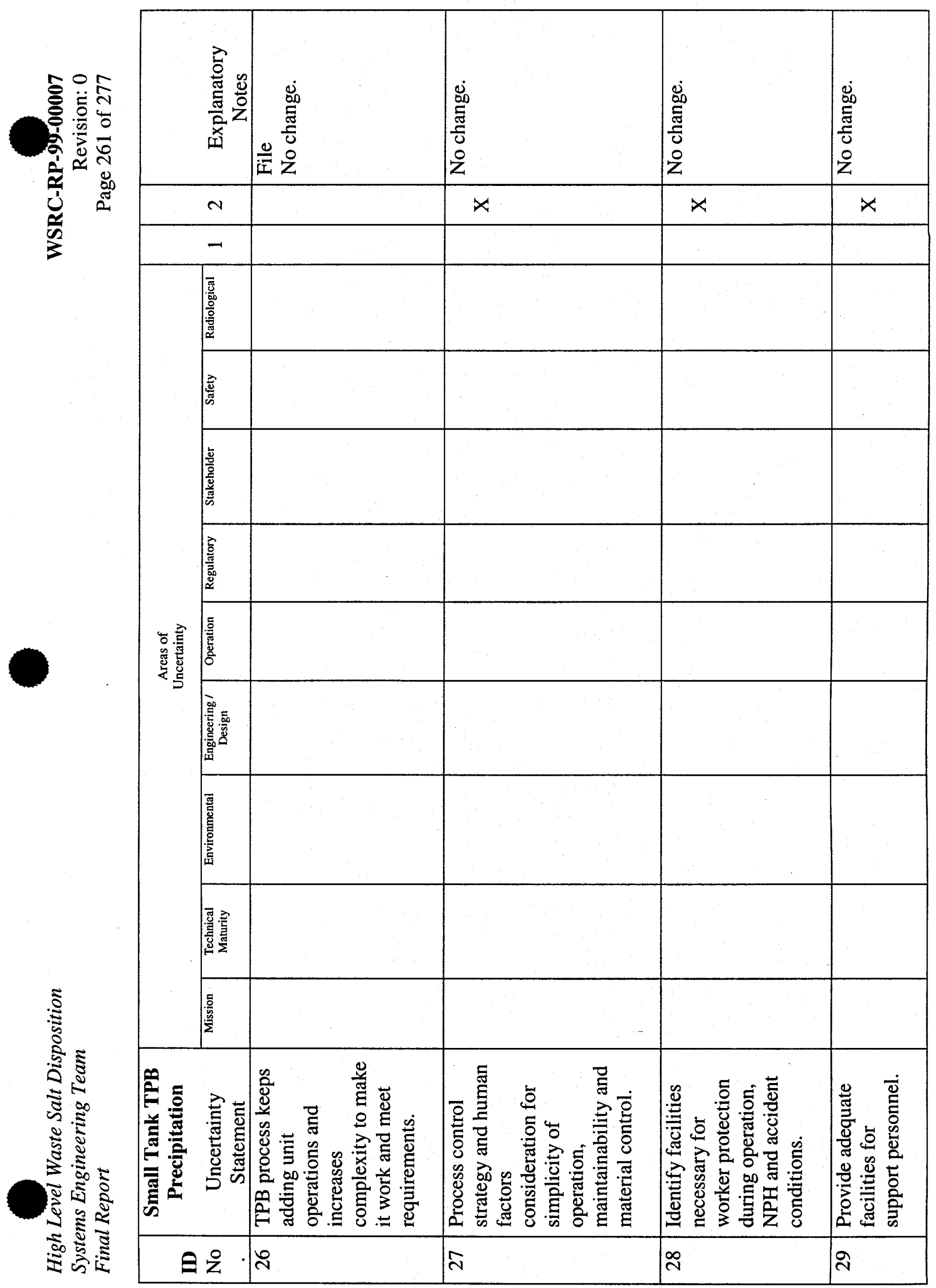


Page 262 of 277

\begin{tabular}{|c|c|c|c|c|c|c|c|c|c|c|c|c|c|}
\hline \multirow{2}{*}{$\begin{array}{l}\text { ID } \\
\text { No }\end{array}$} & \multirow{2}{*}{$\begin{array}{c}\text { Small Tank TPB } \\
\text { Precipitation } \\
\text { Uncertainty } \\
\text { Statement } \\
\end{array}$} & \multicolumn{9}{|c|}{$\begin{array}{l}\text { Areas of } \\
\text { Uncertainty }\end{array}$} & \multirow[b]{2}{*}{1} & \multirow[b]{2}{*}{2} & \multirow[b]{2}{*}{$\begin{array}{c}\text { Explanatory } \\
\text { Notes }\end{array}$} \\
\hline & & Mission & $\begin{array}{l}\text { Technical } \\
\text { Maturity }\end{array}$ & Environmental & $\begin{array}{l}\text { Engineering / } \\
\text { Design }\end{array}$ & Operation & Regulatory & Stakeholder & Safety & Radiological & & & \\
\hline 30 & $\begin{array}{l}\text { Hazards } \\
\text { Analysis/PHR may } \\
\text { require isolated two } \\
\text { train design. }\end{array}$ & . & & & & & & & & & & $\mathrm{X}$ & No change. \\
\hline 31 & $\begin{array}{l}\text { Are we doing } \\
\text { anything to keep } \\
\text { sludge out of the } \\
\text { process? Does } \\
\text { doing the MST } \\
\text { strike and sludge } \\
\text { removal provide } \\
\text { process benefits as } \\
\text { a separate head end } \\
\text { unit operation. }\end{array}$ & & & & & & & & & & & & $\begin{array}{l}\text { File } \\
\text { No change. } \\
\text { HLW-SDT-99- } \\
\text { 0289 } \\
\text { WSRC-TR-99- } \\
00208\end{array}$ \\
\hline 32 & $\begin{array}{l}\text { Does sample } \\
\text { efficacy cover the } \\
\text { materials that can } \\
\text { get you in trouble? }\end{array}$ & & & & & & & & & & & & $\begin{array}{l}\text { File } \\
\text { No change. }\end{array}$ \\
\hline 33 & $\begin{array}{l}\text { Design } \\
\text { considerations for } \\
\text { radiological } \\
\text { operations and } \\
\text { response. }\end{array}$ & & & & & & & & & & & & $\begin{array}{l}\text { File } \\
\text { No change. }\end{array}$ \\
\hline
\end{tabular}




\begin{tabular}{|c|c|c|c|c|c|c|c|c|c|c|c|c|c|}
\hline \multirow{2}{*}{$\begin{array}{l}\text { ID } \\
\text { No }\end{array}$} & \multirow{2}{*}{$\begin{array}{c}\text { Small Tank TPB } \\
\text { Precipitation } \\
\text { Uncertainty } \\
\text { Statement } \\
\end{array}$} & \multicolumn{9}{|c|}{$\begin{array}{l}\text { Areas of } \\
\text { Uncertainty }\end{array}$} & \multirow[b]{2}{*}{1} & \multirow[b]{2}{*}{2} & \multirow[b]{2}{*}{$\begin{array}{c}\text { Explanatory } \\
\text { Notes }\end{array}$} \\
\hline & & Mission & $\begin{array}{l}\text { Technical } \\
\text { Maturity }\end{array}$ & Environmental & $\begin{array}{c}\text { Engineering } 1 \\
\text { Design }\end{array}$ & Operation & Regulatory & Stakeholder & Safety & Radiological & & & \\
\hline 34 & $\begin{array}{l}\text { Tank recovery } \\
\text { strategy (Tank } 48 \\
\text { and } 49 \text { ) to support } \\
\text { operations. }\end{array}$ & & & & & & & & & & & & $\begin{array}{l}\text { File } \\
\text { No change. } \\
\text { WSRC-RP-99- } \\
00005\end{array}$ \\
\hline 35 & $\begin{array}{l}\text { Filter blinding from } \\
\text { gas entrainment and } \\
\text { pressure drop in the } \\
\text { filter assembly. }\end{array}$ & & & & & & & & & & & $X$ & $\begin{array}{l}\text { No change. } \\
\text { WSRC-TR-99- } \\
00243 \\
\text { ORNL/TM- } \\
\text { 1999/234 }\end{array}$ \\
\hline 36 & $\begin{array}{l}\text { What have we done } \\
\text { to address the scale- } \\
\text { up and variable } \\
\text { materials for TPB } \\
\text { hydrolysis and } \\
\text { DWPF. }\end{array}$ & & 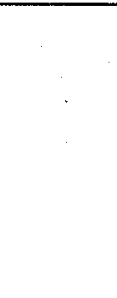 & & & & & & & & & $X$ & $\begin{array}{l}\text { WSRC-TR-99- } \\
00272\end{array}$ \\
\hline 37 & $\begin{array}{l}\text { Will CSTR } \\
\text { negatively impact } \\
\text { filtration rate. } \\
\text { Based on fresh } \\
\text { precipitate and } \\
\text { particle size at } 25 \\
{ }^{\circ} \mathrm{C} \text {. }\end{array}$ & & & & & & & & & & & $X$ & $\begin{array}{l}\text { ORNL/TM- } \\
\text { 1999/234 } \\
\text { WSRC-TR-99- } \\
00243\end{array}$ \\
\hline
\end{tabular}




\begin{tabular}{|c|c|c|c|c|c|c|c|c|c|c|c|c|c|}
\hline \multirow{2}{*}{$\begin{array}{l}\text { ID } \\
\text { No }\end{array}$} & \multirow{2}{*}{$\begin{array}{c}\text { Small Tank TPB } \\
\text { Precipitation } \\
\text { Uncertainty } \\
\text { Statement } \\
\end{array}$} & \multicolumn{9}{|c|}{$\begin{array}{l}\text { Areas of } \\
\text { Uncertainty }\end{array}$} & \multirow[b]{2}{*}{1} & \multirow[b]{2}{*}{2} & \multirow[b]{2}{*}{$\begin{array}{l}\text { Explanatory } \\
\text { Notes }\end{array}$} \\
\hline & & Mission & $\begin{array}{l}\text { Technical } \\
\text { Maturity }\end{array}$ & Environmental & $\begin{array}{c}\text { Engineering / } \\
\text { Design }\end{array}$ & Operation & Regulatory & Stakeholder & Safety & $\begin{array}{l}\text { Radiological } \\
\end{array}$ & & & \\
\hline 38 & $\begin{array}{l}\text { Precipitate } \\
\text { hydrolysis will be } \\
\text { idle for } 10 \text { years. } \\
\text { Equipment } \\
\text { operational } \\
\text { readiness. }\end{array}$ & & & & & & & & & & & & $\begin{array}{l}\text { File } \\
\text { No change. } \\
\text { HLW-SDT-99- } \\
0266 \\
\text { WSRC-RP-99- } \\
00006 \\
\text { WSRC-RP-99- } \\
\text { 00005 }\end{array}$ \\
\hline 39 & $\begin{array}{l}\text { Radioactive waste } \\
\text { tests to support } \\
\text { equipment design } \\
\text { and confirm cold } \\
\text { test results for } \\
\text { chemical analysis. }\end{array}$ & . & & & & & & & & & & & $\begin{array}{l}\text { File } \\
\text { No change. } \\
\text { WSRC-TR-99- } \\
00345\end{array}$ \\
\hline 40 & $\begin{array}{l}\text { Additional antifoam } \\
\text { will have } \\
\text { deleterious effects } \\
\text { on downstream } \\
\text { processes. }\end{array}$ & & $\mathrm{X}$ & . & & & & & & & $\mathrm{X}$ & & $\begin{array}{l}\text { Real waste test } \\
\text { suggest the need } \\
\text { for a different } \\
\text { anti-foam. } \\
\text { WSRC-TR-99- } \\
00345\end{array}$ \\
\hline
\end{tabular}


Page 265 of 277

\begin{tabular}{|c|c|c|c|c|c|c|c|c|c|c|c|c|c|}
\hline \multirow{2}{*}{$\begin{array}{l}\text { ID } \\
\text { No }\end{array}$} & \multirow{2}{*}{$\begin{array}{c}\text { Small Tank TPB } \\
\text { Precipitation } \\
\text { Uncertainty } \\
\text { Statement }\end{array}$} & \multicolumn{9}{|c|}{$\begin{array}{l}\text { Areas of } \\
\text { Uncertainty }\end{array}$} & \multirow[b]{2}{*}{1} & \multirow[b]{2}{*}{2} & \multirow[b]{2}{*}{$\begin{array}{c}\text { Explanatory } \\
\text { Notes }\end{array}$} \\
\hline & & Mission & $\begin{array}{l}\text { Technical } \\
\text { Maturity }\end{array}$ & Environmental & $\begin{array}{c}\text { Engineering/ } \\
\text { Designg }\end{array}$ & Operation & Regulatory & Stakeholder & Safety & Radiological & & & \\
\hline 41 & $\begin{array}{l}\mathrm{Np} \\
\text { decontamination } \\
\text { may not be } \\
\text { adequate to meet } \\
\text { Saltstone } \\
\text { Performance } \\
\text { Assessment (PA). }\end{array}$ & & & & & & & & & & & & $\begin{array}{l}\text { File } \\
\text { No change. }\end{array}$ \\
\hline$\overline{42}$ & $\begin{array}{l}\text { TRU } \\
\text { decontamination } \\
\text { may require } \\
\text { excessive MST. }\end{array}$ & & $X$ & & & " & & & & & $X$ & & $\begin{array}{l}\text { Equilibrium } \\
\text { capacity shown to } \\
\text { be sufficient by } \\
\text { R\&D. Neptunium } \\
\text { for Tanks } 33 \text { and } \\
34 \text { will require } \\
\text { blending. } \\
\text { WSRC-TR-99- } \\
\text { O0219 } \\
\text { WSRC-TR-99- } \\
\text { 00286 } \\
\text { No change. }\end{array}$ \\
\hline 43 & $\begin{array}{l}\text { Process will not } \\
\text { produce the } \mathrm{dF} \\
\text { required because of } \\
\text { slow kinetics of } \\
\text { MST and TPB. }\end{array}$ & & & & & & & & & & & $\bar{X}$ & $\begin{array}{l}\text { ORNL/TM- } \\
\text { 1999/234 } \\
\text { WSRC-TR-99- } \\
00345\end{array}$ \\
\hline
\end{tabular}


Page 266 of 277

\begin{tabular}{|c|c|c|c|c|c|c|c|c|c|c|c|c|c|}
\hline \multirow{2}{*}{$\begin{array}{l}\text { ID } \\
\text { No }\end{array}$} & \multirow{2}{*}{$\begin{array}{c}\text { Small Tank TPB } \\
\text { Precipitation } \\
\text { Uncertainty } \\
\text { Statement }\end{array}$} & \multicolumn{9}{|c|}{$\begin{array}{l}\text { Areas of } \\
\text { Uncertainty }\end{array}$} & \multirow[b]{2}{*}{1} & \multirow[b]{2}{*}{2} & \multirow[b]{2}{*}{$\begin{array}{c}\text { Explanatory } \\
\text { Notes }\end{array}$} \\
\hline & & Mission & $\begin{array}{l}\text { Technical } \\
\text { Maturity }\end{array}$ & Environmental & $\begin{array}{l}\text { Engineering } 1 \\
\text { Design }\end{array}$ & Operation & Regulatory & Stakeholder & Safety & Radiological & & & \\
\hline 44 & $\begin{array}{l}\text { The precipitate will } \\
\text { be difficult to filter. }\end{array}$ & & & & & & & & & & & & $\begin{array}{l}\text { File } \\
\text { No change. } \\
\text { WSRC-TR-99- } \\
\text { 00345 } \\
\text { ORNL/TM- } \\
\text { 1999/234 }\end{array}$ \\
\hline 45 & $\begin{array}{l}\text { Distribution of } \\
\text { byproducts of } \\
\text { hydrolysis reaction } \\
\text { could be deleterious } \\
\text { to DWPF. }\end{array}$ & & & & & & & & & & & $\mathrm{X}$ & No change. \\
\hline 46 & $\begin{array}{l}\text { Enough recycle } \\
\text { organics from } \\
\text { (DWPF) Salt Cell } \\
\text { will exist to } \\
\text { negatively affect } \\
\text { the Tank Farm. }\end{array}$ & & 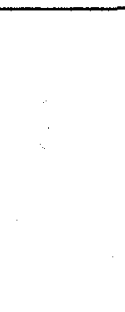 & & & & & & & & & $X$ & No change. \\
\hline 47 & $\begin{array}{l}\text { HLW cannot } \\
\text { support tank } \\
\text { blending strategies } \\
\text { for Cs (or other } \\
\text { species) to support } \\
\text { process } \\
\text { requirements. }\end{array}$ & & & & & & & & & & & $\mathrm{X}$ & No change. \\
\hline
\end{tabular}




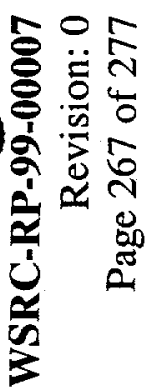

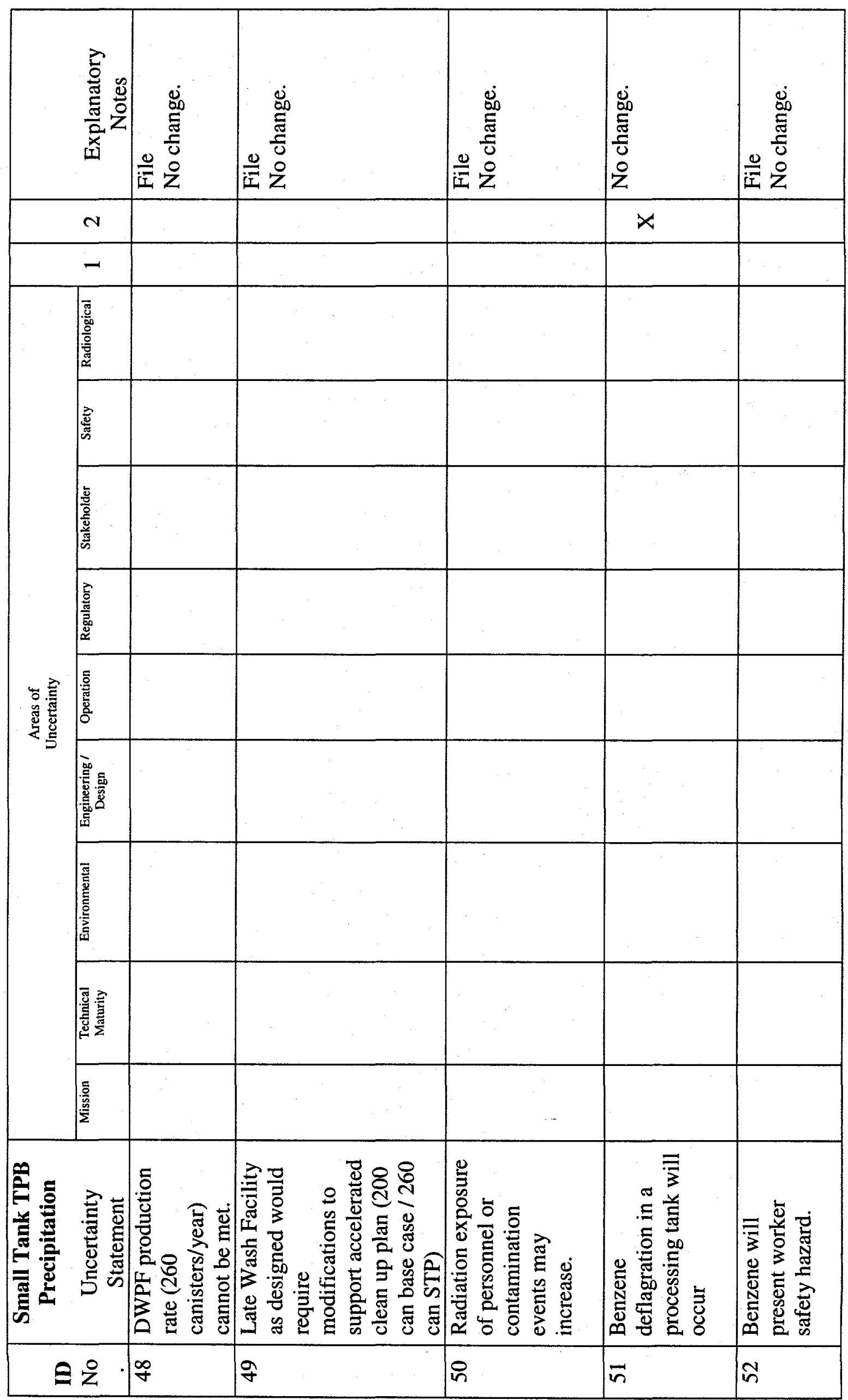




\begin{tabular}{|c|c|c|c|c|c|c|c|c|c|c|c|c|c|}
\hline \multirow{2}{*}{$\begin{array}{l}\text { ID } \\
\text { No }\end{array}$} & \multirow{2}{*}{$\begin{array}{c}\text { Small Tank TPB } \\
\text { Precipitation } \\
\text { Uncertainty } \\
\text { Statement } \\
\end{array}$} & \multicolumn{9}{|c|}{$\begin{array}{l}\text { Areas of } \\
\text { Uncertainty }\end{array}$} & \multirow[b]{2}{*}{1} & \multirow[b]{2}{*}{2} & \multirow[b]{2}{*}{$\begin{array}{l}\text { Explanatory } \\
\text { Notes }\end{array}$} \\
\hline & & Mission & $\begin{array}{l}\text { Technical } \\
\text { Maturity }\end{array}$ & Environmental & $\begin{array}{c}\text { Engineering / } \\
\text { Design }\end{array}$ & Operation & Regulatory & Stakeholder & Safety & Radiological & & & \\
\hline 53 & $\begin{array}{l}\text { Process chemistry } \\
\text { understanding and } \\
\text { application are still } \\
\text { under development, } \\
\text { resulting in } 96-1 \\
\text { lessons learned not } \\
\text { yet implemented. }\end{array}$ & & $x$ & & & & & & & & $X$ & & $\begin{array}{l}\text { Catalyst } \\
\text { activation greater } \\
\text { than the bounding } \\
\text { case results in a } \\
\text { loss of product } \\
\text { DF. } \\
\text { WSRC-TR-99- } \\
00279\end{array}$ \\
\hline 54 & $\begin{array}{l}\text { Cannot design } \\
\text { adequate safety } \\
\text { features to protect } \\
\text { for all benzene } \\
\text { generation rate(s). }\end{array}$ & & & & & & & & & & & & $\begin{array}{l}\text { File } \\
\text { No change. }\end{array}$ \\
\hline 55 & $\begin{array}{l}\text { Stakeholders will } \\
\text { reject the } \\
\text { alternative }\end{array}$ & & & & & & & & & & & & $\begin{array}{l}\text { File } \\
\text { No change. }\end{array}$ \\
\hline 56 & $\begin{array}{l}\text { MST amount } \\
\text { needed for } \\
\text { decontamination } \\
\text { exceeds DWPF Ti } \\
\text { glass limits. }\end{array}$ & & & & & & & & & & & $\mathrm{X}$ & $\begin{array}{l}\text { MST concentrate } \\
\text { of } 0.4 \mathrm{~g} / \mathrm{L} \text { makes } \\
\text { acceptable glass. } \\
\text { WSRC-TR-99- } \\
00332\end{array}$ \\
\hline 57 & $\begin{array}{l}\text { GT73 resin will be } \\
\text { selective to Co60 or } \\
\text { other trace } \\
\text { radioisotopes. }\end{array}$ & & & & & & & & & & & & File \\
\hline
\end{tabular}




\begin{tabular}{|c|c|c|c|c|c|c|c|c|c|c|c|c|c|}
\hline \multirow{2}{*}{$\begin{array}{l}\text { ID } \\
\text { No }\end{array}$} & \multirow{2}{*}{$\begin{array}{c}\text { Small Tank TPB } \\
\text { Precipitation } \\
\text { Uncertainty } \\
\text { Statement }\end{array}$} & \multicolumn{9}{|c|}{$\begin{array}{l}\text { Areas of } \\
\text { Uncertainty }\end{array}$} & \multirow[b]{2}{*}{1} & \multirow[b]{2}{*}{2} & \multirow[b]{2}{*}{$\begin{array}{c}\text { Explanatory } \\
\text { Notes }\end{array}$} \\
\hline & & Mission & $\begin{array}{l}\text { Technical } \\
\text { Maturity }\end{array}$ & Environmental & $\begin{array}{l}\text { Engineering / } \\
\text { Design }\end{array}$ & Operation & Regulatory & Stakeholder & Safety & Radiological & & & \\
\hline 58 & $\begin{array}{l}\text { Mercury removal } \\
\text { resin (GT-73) will } \\
\text { not work in high } \\
\text { caustic environment } \\
(>2 \text { Molar). } \\
\end{array}$ & & & & & & & & & & & & File \\
\hline 59 & $\begin{array}{l}\text { Accumulation of } \\
\text { hydrogen may } \\
\text { occur in vapor } \\
\text { spaces (including } \\
\text { process vessels) } \\
\text { and } \\
\text { deflagrations/deton } \\
\text { ation could occur if } \\
\text { there is a spark } \\
\text { source. }\end{array}$ & & & & & & & & & & & $X$ & No change. \\
\hline 60 & $\begin{array}{l}\text { The neptunium } \\
\text { content in certain } \\
\text { HLW Tanks may } \\
\text { impact the PA and } \\
\text { WAC. }\end{array}$ & & & & & & & & & & & & $\begin{array}{l}\text { File } \\
\text { No change. } \\
\text { Neptunium for } \\
\text { Tanks } 33 \text { and } 34 \\
\text { will require } \\
\text { blending. } \\
\text { WSRC-TR-99- } \\
00219 \\
\text { WSRC-TR-99- } \\
00286 \\
\end{array}$ \\
\hline
\end{tabular}




\begin{tabular}{|c|c|c|c|c|c|c|c|c|c|c|c|c|c|}
\hline \multirow{2}{*}{$\begin{array}{l}\text { ID } \\
\text { No }\end{array}$} & \multirow{2}{*}{$\begin{array}{c}\text { Small Tank TPB } \\
\text { Precipitation } \\
\text { Uncertainty } \\
\text { Statement }\end{array}$} & \multicolumn{9}{|c|}{$\begin{array}{l}\text { Areas of } \\
\text { Uncertainty }\end{array}$} & \multirow[b]{2}{*}{1} & \multirow[b]{2}{*}{2} & \multirow[b]{2}{*}{$\begin{array}{c}\text { Explanatory } \\
\text { Notes }\end{array}$} \\
\hline & & Mission & $\begin{array}{l}\text { Technical } \\
\text { Maturity }\end{array}$ & Environmental & $\begin{array}{c}\text { Engineering/ } \\
\text { Design }\end{array}$ & Operation & Regulatory & Stakeholder & Safety & Radiological & & & \\
\hline 61 & $\begin{array}{l}\text { Difficulty in } \\
\text { filtration of sludge } \\
\text { and/or MST will } \\
\text { produce low filtrate } \\
\text { flow rates and } \\
\text { require frequent } \\
\text { cleaning }\end{array}$ & & & & & & & & & & & & $\begin{array}{l}\text { File } \\
\text { WSRC-TR-99- } \\
00243 \\
\text { ORNLTM- } \\
\text { 199/234e }\end{array}$ \\
\hline 62 & $\begin{array}{l}\text { Difficulty in } \\
\text { resuspending MST } \\
\text { after long quiescent } \\
\text { period }\end{array}$ & & & & & & & & & & & & File \\
\hline 63 & $\begin{array}{l}\text { Monitoring GT-73 } \\
\text { performance and } \\
\text { breakthrough. }\end{array}$ & & & & & & & & & & & & File \\
\hline 64 & $\begin{array}{l}\text { Temperature } \\
\text { changes or } \\
\text { chemistry changes } \\
\text { may cause post } \\
\text { precipitation after } \\
\text { the MST strike. }\end{array}$ & & & & & & & & & & & & $\begin{array}{l}\text { File } \\
\text { No change. }\end{array}$ \\
\hline 65 & $\begin{array}{l}\text { Hydrogen control } \\
\text { in the MST strike } \\
\text { process. }\end{array}$ & & & & & & & & & & & $\mathrm{X}$ & No change. \\
\hline
\end{tabular}


Page 271 of 277

\begin{tabular}{|c|c|c|c|c|c|c|c|c|c|c|c|c|c|}
\hline \multirow{2}{*}{$\begin{array}{l}\text { ID } \\
\text { No }\end{array}$} & \multirow{2}{*}{$\begin{array}{c}\text { Small Tank TPB } \\
\text { Precipitation } \\
\text { Uncertainty } \\
\text { Statement } \\
\end{array}$} & \multicolumn{9}{|c|}{$\begin{array}{c}\text { Areas of } \\
\text { Uncertainty }\end{array}$} & \multirow[b]{2}{*}{1} & \multirow[b]{2}{*}{2} & \multirow[b]{2}{*}{$\begin{array}{c}\text { Explanatory } \\
\text { Notes }\end{array}$} \\
\hline & & Mission & $\begin{array}{l}\text { Technical } \\
\text { Maturity }\end{array}$ & Environmental & $\begin{array}{l}\text { Engineering / } \\
\text { Design }\end{array}$ & Operation & Regulatory & Stakeholder & Safety & Radiological & & & \\
\hline 66 & $\begin{array}{l}\text { Analysis delay (1 } \\
\text { week) in measuring } \\
\text { for } S_{r} \text { DF in MST } \\
\text { process. }\end{array}$ & & $\mathrm{x}$ & & & & & & & & $\mathrm{X}$ & & $\begin{array}{l}\text { Requires new } \\
\text { analytical } \\
\text { techniques. } \\
\text { No change. }\end{array}$ \\
\hline 67 & $\begin{array}{l}\text { Fate and } \\
\text { downstream impact } \\
\text { of oxalate, after a } \\
\text { cross flow filter } \\
\text { cleaning operation }\end{array}$ & & & & & & & & & 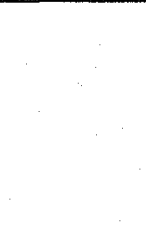 & & & $\begin{array}{l}\text { File } \\
\text { No change. }\end{array}$ \\
\hline 68 & $\begin{array}{l}\text { Rate of spent } \\
\text { equipment } \\
\text { generation and its } \\
\text { disposal. }\end{array}$ & & & & & & & & & & & $X$ & No change. \\
\hline 69 & $\begin{array}{l}\text { Minimize spark } \\
\text { sources in } \mathrm{H}_{2} \text { rich } \\
\text { areas. }\end{array}$ & & & & & & & & & & & $X$ & No change. \\
\hline 70 & $\begin{array}{l}\text { What is the fate of } \\
\text { Am in the process? }\end{array}$ & & $\mathrm{x}$ & & & & & & & & $X$ & & $\begin{array}{l}\text { Does MST strike } \\
\text { affect Am } \\
\text { disposition? } \\
\text { No change. }\end{array}$ \\
\hline
\end{tabular}


Page 272 of 277

\begin{tabular}{|c|c|c|c|c|c|c|c|c|c|c|c|c|c|}
\hline \multirow{2}{*}{$\begin{array}{l}\text { ID } \\
\text { No }\end{array}$} & \multirow{2}{*}{$\begin{array}{c}\text { Small Tank TPB } \\
\text { Precipitation } \\
\text { Uncertainty } \\
\text { Statement } \\
\end{array}$} & \multicolumn{9}{|c|}{$\begin{array}{l}\text { Areas of } \\
\text { Uncertainty }\end{array}$} & \multirow[b]{2}{*}{1} & \multirow[b]{2}{*}{2} & \multirow[b]{2}{*}{$\begin{array}{c}\text { Explanatory } \\
\text { Notes }\end{array}$} \\
\hline & & Mission & $\begin{array}{l}\text { Technical } \\
\text { Maturity }\end{array}$ & Environmental & $\begin{array}{c}\text { Engineering / } \\
\text { Design }\end{array}$ & Operation & Regulatory & Stakeholder & Safety & Radiological & & & \\
\hline 71 & $\begin{array}{l}\text { Existing transfer } \\
\text { lines and tank size } \\
\text { and drain back not } \\
\text { adequate, and leads } \\
\text { to inefficiencies. }\end{array}$ & & & & & & & & & & & $X$ & No change. \\
\hline 72 & $\begin{array}{l}\text { Solids on filters } \\
\text { will not dissolve or } \\
\text { would be difficult } \\
\text { to dissolve in oxalic } \\
\text { acid. }\end{array}$ & & & & & & & & & & & & $\begin{array}{l}\text { File } \\
\text { No change. }\end{array}$ \\
\hline 73 & $\begin{array}{l}\text { Require adequate } \\
\text { size of remote and } \\
\text { contact } \\
\text { decontamination } \\
\text { cells, with adequate } \\
\text { crane coverage. }\end{array}$ & i & & . & & & & & & & & $\mathrm{X}$ & No change. \\
\hline 74 & $\begin{array}{l}\text { Need for docking } \\
\text { door to maintain } \\
\text { clean crane controls } \\
\text { and electronics. }\end{array}$ & & & & & & & & & & & $X$ & No change. \\
\hline
\end{tabular}




\begin{tabular}{|c|c|c|c|c|c|c|c|c|c|c|c|c|c|}
\hline \multirow{2}{*}{$\begin{array}{l}\text { ID } \\
\text { No }\end{array}$} & \multirow{2}{*}{$\begin{array}{c}\text { Small Tank TPB } \\
\text { Precipitation } \\
\text { Uncertainty } \\
\text { Statement } \\
\end{array}$} & \multicolumn{9}{|c|}{$\begin{array}{l}\text { Areas of } \\
\text { Uncertainty }\end{array}$} & \multirow[b]{2}{*}{1} & \multirow[b]{2}{*}{2} & \multirow[b]{2}{*}{$\begin{array}{c}\text { Explanatory } \\
\text { Notes }\end{array}$} \\
\hline & & Mission & $\begin{array}{l}\text { Technical } \\
\text { Maturity }\end{array}$ & Environmental & $\begin{array}{l}\text { Engineering/ } \\
\text { Designg }\end{array}$ & Operation & Regulatory & Stakeholder & Safety & Radiological & & & \\
\hline 75 & $\begin{array}{l}\text { Adequate process } \\
\text { instrumentation to } \\
\text { detect process } \\
\text { upsets and perform } \\
\text { routine monitoring. }\end{array}$ & & & & & & & & & & & $X$ & No change. \\
\hline
\end{tabular}



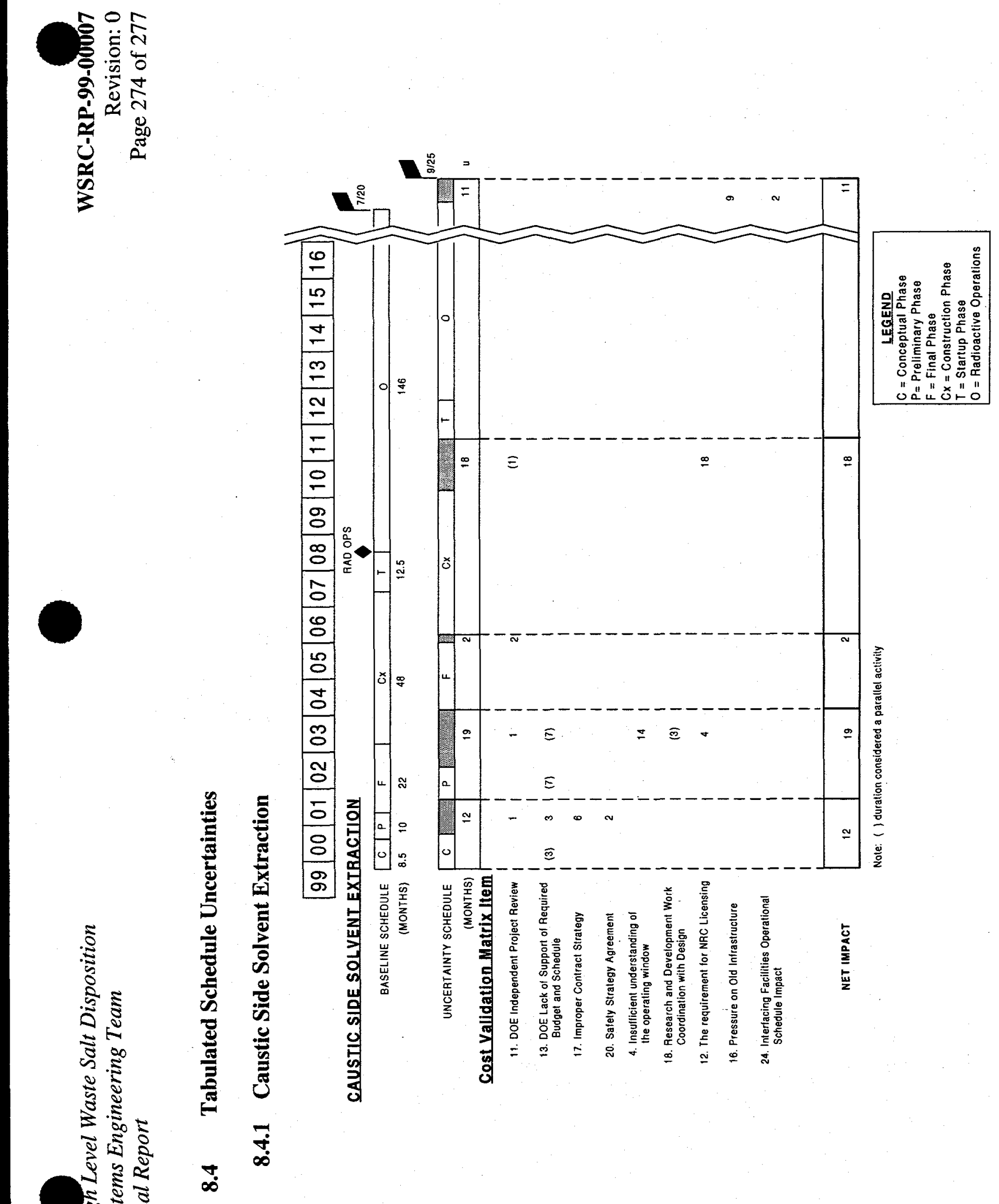


\subsubsection{CST Non-Elutable Ion Exchange}

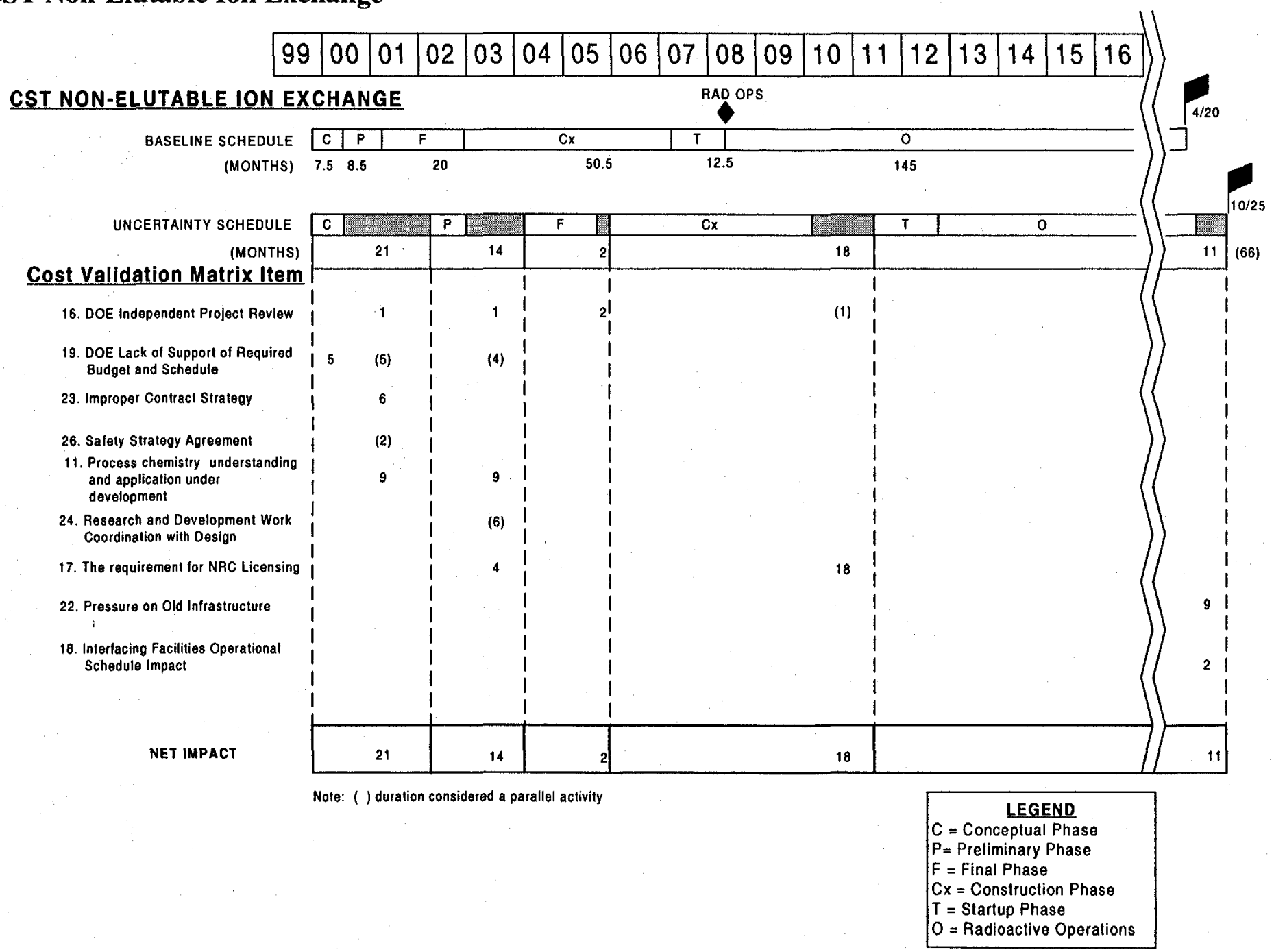




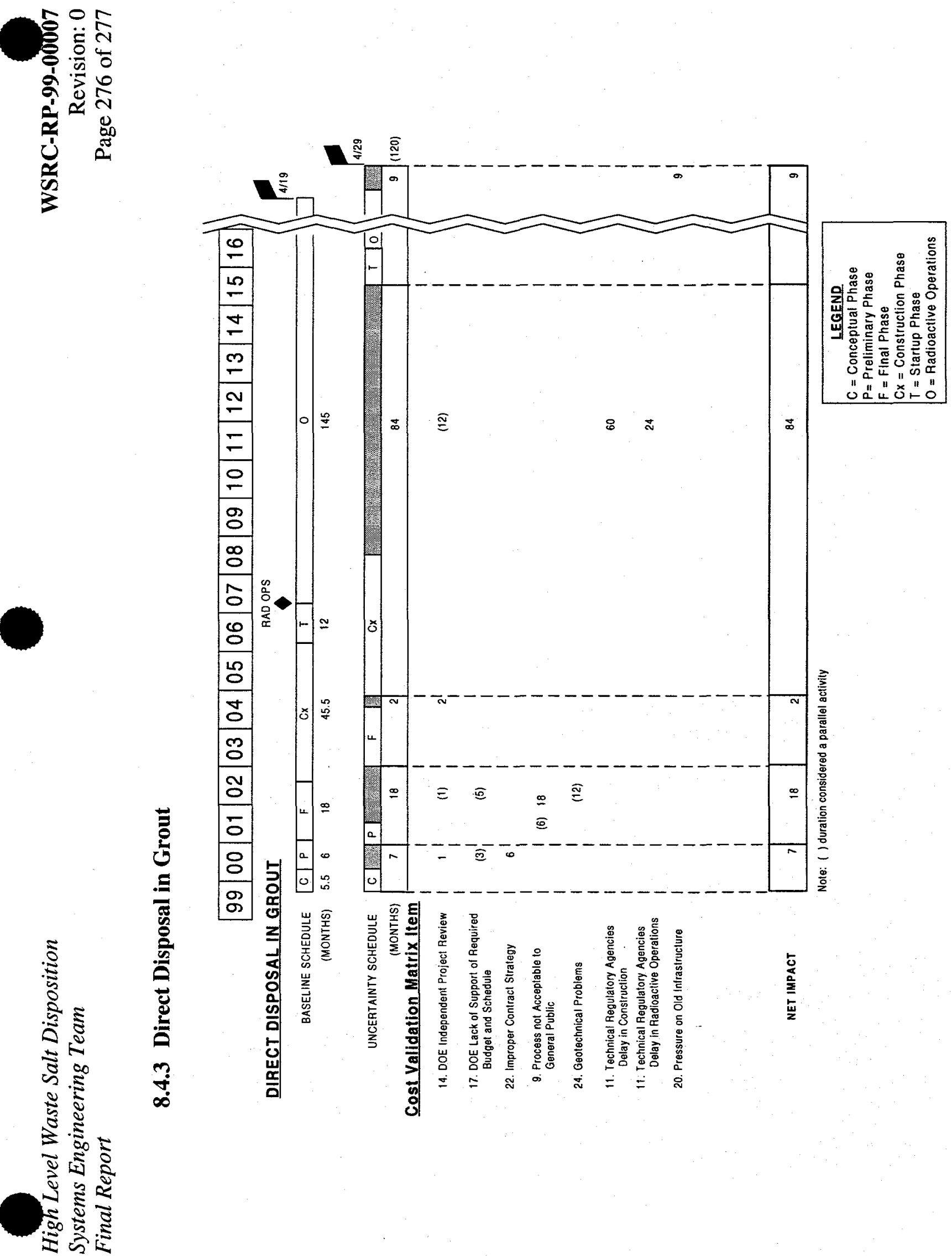



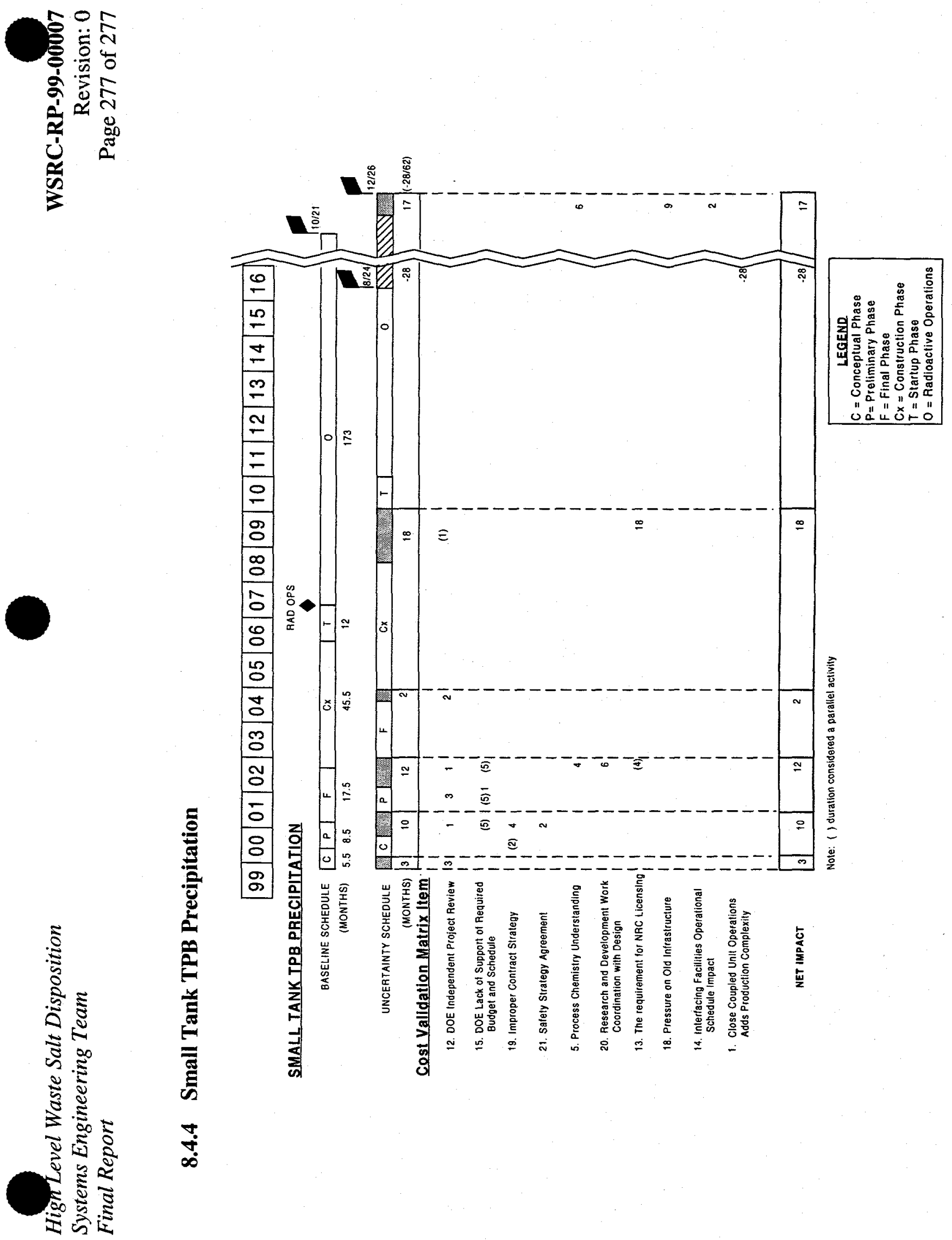